\title{
MEASUREMENT OF THE $M_{A}^{Q E}$ PARAMETER USING MULTIPLE QUASI-ELASTIC DOMINATED SUB-SAMPLES IN THE MINOS NEAR DETECTOR
}

\author{
Nathan S. Mayer
}

Submitted to the faculty of the University Graduate School

in partial fulfillment of the requirements

for the degree

Doctor of Philosophy

in the Department of Physics,

Indiana University

December 2011 
Accepted by the Graduate Faculty, Indiana University, in partial fulfillment of the requirements for the degree of Doctor of Philosophy.

Doctoral Committee

Mark Messier, Ph.D.

Rex Tayloe

Jon Urheim

Mike Snow

Date of Oral Examination

December 5, 2011 
Copyright (C)2011

Nathan Mayer 
For Gloria, who taught me to reach for the stars, but made sure that my feet stayed grounded here on earth. I miss you, Grandma. 


\section{Acknowledgements}

I have been training to be a physicist for the last thirteen years of my life. I've spent so much of my life working to become a scientist that being a scientist is as important to my personal identity as breathing is to sustaining life. The people who's influence helped me to become the person that I am, also shaped me into the physicist that I've become. The same is true of the people who trained me to be the physicist, they have helped to make me the person that I've become.

First I need to thank two people without whom this dissertation would not have been possible. Mark Dorman, who developed the core code that was used to fit for the quasi-elastic axial mass, if it weren't for Mark I would still be writing the infrastructure code. Mark also provided a great deal of assistance, helping me understand the Llewellyn Smith model. During my time at Duluth Aaron Mislivec developed an early version of the algorithm that's used to find candidate proton tracks, with out his early work this analysis would look very different.

I owe more thanks than I could possible express to my advisor Mark Messier. Mark encouraged me to take on an analysis, that was outside of his comfort zone. Than proceeded to find out everything he could about quasi-elastic neutrino interactions, so that I could write the best dissertation I could. His constant encouragement, and feedback have made me a better scientist and better person. If I become half the scientist he already is than I will have become an exceptional scientist.

The first time I applied to graduate school I did so unsuccessfully, with universal denials from every doctoral program the I applied to. I applied to UMD as a hail mary, fortunately the physics department allowed to me register as a graduate 
student that year. Rik was my advisor at UMD, he provided my first real exposure to being a scientist. His unremitting thoroughness for the details of an analysis, though annoying at times ("OK, you know what you should try next...") is exactly what a person like me needed when I was first learning how to be a scientist.

Thanks also go to my defense committee Rex, Mike, and Jon, do to unforeseen circumstances I needed to scramble very near my defense deadline to replace a few of the original committee members. I've worked with all of you in some context over the last few years. You've all taught me quite a lot and for that you have my gratitude.

My eternal appreciation goes to the IU high energy neutrino research group for providing me with the guidance and companionship over the last few years I could not have completed my degree with it. Further thanks go to the group along with the US DOE for providing the funding that allowed me to attend graduate school.

I would never have been able to go from a twelve page analysis note to a two hundred and three page dissertation draft in two and a half months without the support of my girlfriend Jen, her unwavering belief that I could finish the dissertation in time is what kept me going. That same draft would have been much less readable without the weekend that she spent editing it. Thank you.

I've never heard any one describe getting through graduate school as easy, the only thing that makes the experience of graduate school bearable is sharing the experience with others who're going through the same thing. For me those people were the graduate students and young post-docs of Young MINOS: Dan, Mark, Zeynep, Alex H., Alex R., Mhair, Ruth, Chris, Jess, and many others who's names I'm forgetting right now. Whether it's going out for a pint in the evening during collaboration meetings, sharing programming tips, or commiserating about the unreasonable Professor (or Convener) who really isn't unreasonable, I never would have be able to make it through the last couple of years without them.

Most importantly I need to thank my mom and dad (Al and Lorri) and my grandpa and grandma (Marvin and Gloria), they each played more than a small 
part in the person that I have grown into, and the scientist that I hope to one day become. My dad was always willing to talk to me about science, whether it was the mysteries of human consciousness or the weirdness Quantum Mechanics, our conversations inspired a deep curiosity that brought me to science. My mothers deep love of nature and all things green, instilled in me a similar love that drives me to look for the beauty that exists in the natural world and is inherent to it's fundamental laws. In science "failures" greatly outnumber successes, my grandfather's stoicism and quite compassion infused me with the patience to accept and move past these numerous failures while continuing to work towards the infrequent successes. Finally my grandmother (for whom this dissertation is dedicated) always treated me like the adult she new I could be, and when necessary treated me like the child I was (or was acting like). This dissertation is dedicated to her because she wasn't able to see me complete it. 
Nathan S. Mayer

\section{MEASUREMENT OF THE $M_{A}^{Q E}$ PARAMETER USING MULTIPLE QUASI-ELASTIC DOMINATED SUB-SAMPLES IN MINOS}

The Main Injector Neutrino Oscillation Search (MINOS) is a two detector, long baseline neutrino oscillation experiment. The MINOS near detector is an ironscintillator tracking/sampling calorimeter and has recorded the world's largest data set of neutrino interactions in the $0-5 \mathrm{GeV}$ region. This high statistics data set is used to make precision measurements of neutrino interaction cross-sections on iron.

The $Q^{2}$ dependence in charged current quasi-elastic (CCQE) scattering probes the axial and vector structure (form factor) of the nucleon/nuclear target, and nuclear effects in neutrino scattering. Presented here is a study of the MINOS Data that will introduce a method that improves the existing MINOS CCQE analysis. This analysis uses an additional CCQE dominated sub-sample from a different kinematic region to reduce correlations between fit parameters in the existing MINOS CCQE analysis. The measured value of the axial-vector mass is $M_{A}^{Q E}=1.312_{-0.038}^{+0.037}$ (fit) ${ }_{-0.265}^{+0.123}$ (syst.) GeV. 


\section{Contents}

1 Introduction 1

1.1 Introduction . . . . . . . . . . . . . . . 1

1.2 Neutrino Oscillation Theory _. . . . . . . . . . . . 4

1.3 Neutrino Oscillations in MINOS . . . . . . . . . . . . . 5

1.4 Motivation for Neutrino Quasi-Elastic Measurements . . . . . . 8

2 Theory of the Weak Interaction 11

2.1 Weak Interaction Phenomenology . . . . . . . . . . . 11

2.1.1 Fermi's Point-like Four-Fermion Theory of $\beta$-Decay . . 11

2.1.2 The Axial Vector Structure of the Weak Interaction . . . 13

2.1.3 Helicity and Chirality . . . . . . . . . . . . . 16

2.1.4 Electroweak Gauge Theory . . . . . . . . . . . . . . . 18

2.2 Neutrino-Nucleus Scattering . . . . . . . . . . . . . . 23

2.2.1 Neutrino Scattering Off of Nucleons . . . . . . . . . 23

2.2.2 Neutrino-Nucleon Scattering Kinematics . . . . . . . . 24

2.2.3 Nucleon Form Factors and the QE Cross Section . . . . 27

2.2 .4 Nuclear Effects . . . . . . . . . . . . . . . . 32

3 Survey of Current Results 39

3.1 Generalized Method for Measuring $M_{A}^{Q E} \ldots \ldots . \ldots . . \ldots 39$

3.2 Summary of World Average Values . . . . . . . . . . . . . . . . 40

3.3 Survey of $M_{A}^{Q E}$ Measurements . . . . . . . . . . . . 44

3.3.1 Argonne 12-Foot Bubble Chamber . . . . . . . . . 44 
3.3.2 Results From the NOMAD Experiment . . . . . . . 48

3.3.3 Results From the MiniBooNE Experiment . . . . . . . 50

3.4 Resolving the MiniBooNE-NOMAD Discrepancy . . . . . . . 55

4 MINOS Experiment $\quad \mathbf{5 8}$

4.1 The NuMI Beamline . . . . . . . . . . . . . . . . . 58

4.2 The MINOS Detectors . . . . . . . . . . . . . 62

4.2.1 Common Detector Features . . . . . . . . . 63

4.2.2 The Calibration Detector . . . . . . . . . 65

4.2 .3 The Far Detector . . . . . . . . . . . . . 66

4.2 .4 The Near Detector . . . . . . . . . . . . 68

5 MINOS Data and Monte Carlo $\quad 72$

5.1 MINOS Data . . . . . . . . . . . . . . 72

5.1 .1 Reconstruction ............... 72

5.1.2 Calibration .................. 77

5.2 MINOS MC ...................... 83

5.2.1 Beam Re-Weighting . . . . . . . . . 85

6 Event Selection $\quad 91$

$6.1 v_{\mu}$-CC Event Selection . . . . . . . . . . . . . 91

6.1.1 Beam Quality Criteria . . . . . . . . . . . . 92

6.1.2 Near Detector Event Quality Criteria: . . . . . . . . 93

6.1.3 Charged Current Preselection . . . . . . . . . . . 94

6.1.4 $v_{\mu}$-CC Multivariate Identification Parameter . . . . . . 95

6.2 Partial Proton Track Reconstruction . . . . . . . . . . . . . . . 99

6.2.1 Reconstruction Algorithm . . . . . . . . . . . . . 100

6.3 The Interaction Sub-Sample Selections . . . . . . . . . . . . . 104

6.3.1 Low Hadronic Energy Quasi-Elastic Like Selection . . . 105

6.3.2 Two Track Quasi-Elastic Like Selection . . . . . . . . . 107

6.3.3 Two Track Background Like Selection . . . . . . . . . . 107 
6.3.4 Selection Purity and Efficiency . . . . . . . . . . 109

6.4 Comparisons of Data to Monte Carlo . . . . . . . . . . . . . 110

6.4.1 Nuclear Correlations in MINOS . . . . . . . . . . 118

7 Extracting the Axial-Vector Mass 120

7.1 Fitting Procedure and Fit Parameters . . . . . . . . . . . . 120

7.2 Mock Data Analysis . . . . . . . . . . . . . . . . 126

7.2.1 Mock Data Construction . . . . . . . . . . 126

7.2.2 Mock Data Fit Results . . . . . . . . . . . . 127

7.2 .3 Conclusion . . . . . . . . . . . . . 128

7.3 Initial Fit to Data . . . . . . . . . . . . . . . 128

7.4 Fit Using The Resonance Suppression Function . . . . . . . . . 133

7.5 New Fit Procedure . . . . . . . . . . . . . . . . . . . 137

7.5.1 Fit Steps . . . . . . . . . . . . . . . 137

7.5.2 Hadronic Shower Energy Offset . . . . . . . . . 139

7.5 .3 Fit Results . . . . . . . . . . . . . . 140

7.5.4 Systematic Error Contribution . . . . . . . . . . 141

7.5.5 Best Fit Confidence Regions . . . . . . . . . . . . 154

7.6 Conclusion . . . . . . . . . . . . . . . . . . 159

8 Interpreting the Results $\quad 160$

8.1 Nuclear Effects . . . . . . . . . . . . . . . . . . 160

8.2 Analysis Improvements . . . . . . . . . . . . . . . . 162

8.2.1 Selection Criteria Systematic . . . . . . . . . 162

8.2.2 Background Modeling . . . . . . . . . . . 164 


\section{List of Figures}

1.1 Survival probability of muon neutrinos as a function of energy given the MINOS baseline and best fit values for the oscillation parameters. The first minimum from the right is the dip that MINOS is most sensitive to. . . . . . . . . . . . . . . .

1.2 From [10]. Top: The MINOS energy spectra of fully reconstructed events in the Far Detector classified as charged current interactions. The dashed histogram represents the spectrum predicted from measurements in the Near Detector assuming no oscillations, while the solid histogram reflects the best fit of the oscillation hypothesis. The shaded area shows the predicted neutral current background. Bottom: The points with error bars are the background-subtracted ratios of data to the no-oscillation hypothesis. Lines show the best fits for: oscillations, decay [11], and decoherence [12] . . . . . . . . . . . . .

1.3 From [10]. Likelihood contours of $68 \%$ and $90 \%$ C.L. around the best fit values for the mass splitting and mixing angle. Also shown are contours from previous measurements $[13,14]$. . .

1.4 The expected 1 and $2 \sigma$ measurements of $\sin ^{2}\left(2 \theta_{23}\right)$ for 6 years of NOvA running ( 3 yrs in neutrino mode +3 yrs in anti-neutrino mode) using numu quasi elastic events in NOvA. The input $\Delta m^{2}$ is taken to coincide with recent MINOS measurements and the three choices of mixing angle are made consistent with the data from Super-Kamiokande ( $>0.92$ at $90 \%$ confidence limit). . . 
2.1 Feynman diagram of the muon decay process $\left(\mu^{-} \rightarrow v_{\mu}+\bar{v}_{e} e^{-}\right)$

2.2 $\mathrm{CC} v_{\mu}-N$ scattering kinematics. Inside the red boxes are the variables that are measured in the MINOS detectors (in the lab frame). The diagram also labels the four-momentum of the particles in the center of mass frame. The Bjorken scaling variable, $x$, denotes the fraction of the nucleon momentum carried by the struck quark. . . . . . . . . . . . . . . . .

2.3 Feynman diagram of the $v_{\mu}$-CC QE interaction, showing both the $\mathrm{v}_{\mu}, W^{+}, \mu^{-}$and the $n, W^{+}, p$ vertices. . . . . . . . .

2.4 Above: quasi-elastic differential cross section off of a free nucleon with respect to $Q^{2}$. Each distribution corresponds to a different value of the axial-vector mass parameter. Below: The same $Q^{2}$ distribution as above except the red and blue distributions are normalized to the area of the black $Q^{2}$ distribution. .

2.5 The CC- $-v_{\mu}$ QE differential cross section with respect to $Q^{2}$ with several values of $k_{F}$ compared to the free nucleon case, shows the low $Q^{2}$ suppression characteristic of Pauli-blocking. Taken from a talk given by M. Sakuda at the 2005 NuFact conference.

2.6 The MiniBooNE $v_{\mu}$ CCQE cross section as a function of the incident neutrino energy. The points are the MiniBooNE data, the red line is the $v_{\mu}$ CCQE cross section with an $M_{A}^{Q E}$ value of $1.02 \mathrm{GeV}$ and including the effect of the most important types of SRC interactions. The black dashed line is the same $v_{\mu}$ CCQE cross section without the SRT interactions. There is remarkable agreement between the MiniBooNE data and the $Q E+n p-n h$ model. This agreement indicates that the $20-30 \%$ discrepancy in the recent high statistics interaction experiments could, in part, be due to these SRC type interactions. . . . . . . . . . 
3.1 $Q^{2}$ distribution from [42]. The data corresponds to the shown histogram, the solid curve corresponds to the dipole axial-vector form factor utilizing the best fit from the flux independent analysis from Table 3.2. The dotted curve corresponds to an alternate form of the axial-vector form factor with $M_{A}^{Q E}=1.11 \mathrm{GeV}$. . .

3.2 From [52]. Comparison the NOMAD $\left\langle\sigma_{q e l}\right\rangle_{v_{\mu}}$ measurements as a function of the neutrino energy in the 1-track and 2-track subsamples. . . . . . . . . . . . . . .

3.3 From [31]. Reconstructed $Q^{2}$ for $v_{\mu}$ CCQE events including systematic errors. The simulation, before (dashed) and after (solid) the fit, is normalized to data. The dotted (dot-dash) curve shows backgrounds that are not CCQE (not "CCQE-like"). The inset shows the $1 \sigma \mathrm{CL}$ contour for the best-fit parameters (star), along with the starting values (circle), and fit results after varying the background shape (triangle). . . . . . . . . .

3.4 From [34]. Distribution of events in $Q^{2} \mathrm{QE}$ for the (a) 2 and (b) 3 subevent samples before the application of the CC1 background correction. Data and MC samples are shown along with the individual $\mathrm{MC}$ contributions from $\mathrm{CCQE}, \mathrm{CC} 1 \pi$, and other channels. In (b), the dashed line shows the $\mathrm{CC} 1 \pi$ reweighting function (with the $y$-axis scale on the right) as determined from the background t procedure. . . . . . . . . . . . . 
3.5 From [34]. Flux-unfolded MiniBooNE $v_{\mu}$ CCQE cross section per neutron as a function of neutrino energy. In (a), shape errors are shown as shaded boxes along with the total errors as bars. In (b), a larger energy range is shown along with results from the LSND [61] and NOMAD [52] experiments. Also shown are predictions from the NUANCE simulation for an RFG model with two different parameter variations and for scattering from free nucleons with the world-average MA value. Numerical values are provided in Table $\mathrm{X}$ in the appendix (of [34]). . . . . . . .

3.6 From [62]. Left: The QE differential cross section $\left(\mathrm{d} \sigma / \mathrm{d} Q^{2}\right)$ as a function of $Q^{2}$ for $v_{\mu}, \bar{v}_{\mu}$ energies of $1.0 \mathrm{GeV}$ (maximum accessible $\left.Q_{\max }^{2}=1.3(\mathrm{GeV} / \mathrm{c})^{2}\right)$. Here, the orange dotted line is the prediction of the "Independent Nucleon $\left(M_{A}=1.014\right)$ " model. The blue dashed line is the prediction of the the "Larger $M_{A}$ $\left(M_{A}=1.3\right)$ " model. The red line is prediction of the "Transverse Enhancement" model. Top (a): $v_{\mu}$ differential QE cross sections. Bottom (b): $\bar{v}_{\mu}$ differential QE cross sections. Right: Same as Left for $v_{\mu}, \bar{v}_{\mu}$ energies of $3.0 \mathrm{GeV}$ (maximum accessible $\left.Q_{\max }^{2}=4.9(\mathrm{GeV} / \mathrm{c})^{2}\right)$. . . . . . . . . . . 56

4.1 From [66]. Geograpical view of the MINOS experimental layout. ......................... 59

4.2 From [66]. The layout of the various FNAL proton accelerators. 60

4.3 From [66]. The NuMI target. . . . . . . . . . . . . . . 60

4.4 From [66]. Left: Photograph of inner conductors of the NuMI parabolic focusing horn. Right: Photograph of the first focusing horn. ........................... 61

4.5 From [66]. The various components of the NuMI beamline. . . 62

4.6 From [66]. Left: Individual scintillator strip. Right: Strips that have collected into a detector module. . . . . . . . . . 64

4.7 From [66]. Schematic of a scintillator plane readout system. . 65 
4.8 From [66]. Photograph of the MINOS Calibration detector. . . 66

4.9 From [66]. Photograph of the completed Far detector. . . . . . 67

4.10 From [66]. Photograph of the Near detector. . . . . . . . . . . 69

4.11 From [65]. Strip configuration of the MINOS ND, divided into partially instrumented (above) and fully instrument (below)planes, and also U-view (left) and V-view (right) planes. . . . . . . . .

5.1 From [65]. Stopping power for muons. The gray line shows the Bethe-Bloch calculation of the stopping power for muons in polystyrene scintillator. The solid circles and open triangles show the response of stopping muons in the far detector data and GEANT3 Monte Carlo simulations respectively. Both data and Monte Carlo points have been normalized to the Bethe-Bloch calculation to give the expected stopping power at the minimum ionizing point. . . . . . . . . . . . .

5.2 From [71]. Variations in the median signal per plane deposited by through-going cosmic-ray muons observed during the datataking period covered by this paper. The time dependence is largely due to variations in the environmental conditions in the Near and Far Detector halls and aging of the scintillator. The zero point on the ordinate is arbitrary. . . . . . . . .

5.3 From [65]. Nonlinearity calibration of near and far detector instrumentation. The intrinsic nonlinearity of PMT response (circles) and its residual after calibration (error bars only) are shown for the near (a) and far (b) detectors. The error bars depict the rms spread of channels in each detector. For scale, a minimum ionizing particle normal to the plane will generate roughly 500600 ADCs of charge each detector. A single photoelectron is roughly 75 ADCs (Far) or 100 ADCs (Near). . . . . . . . . . 
5.4 From [65]. Mean value of the strip-end responses normalized to the detector average. The mean response of the strip ends varies by approximately $29 \%$. The solid and dashed lines are the calculated responses of two separate data sets from June 2005. The statistical variation in the individual calibration constants from these two data sets is on the order of $2.1 \%$. . . . . . .

5.5 From [65]. Comparison of cosmic ray muon data (points) with module mapper fitting results (solid curve) for a typical strip in the near detector. . . . . . . . . . . . . . . .

5.6 From [65]. MINOS calorimetric response to pions and electrons at three momenta. The calorimeter-signal scale is in arbitrary units. The data (open symbols), obtained from the calibration detector exposure to CERN test beams, are compared to distributions from Monte Carlo simulations. . . . . . . . . . . .

5.7 From [33][71]. The NEUGEN calculated cross sections for the $\mathrm{v}_{\mu}$-CC inclusive, QE scattering and single pion production as a function of neutrino energy. These cross sections are calculated assuming an isoscalar averaged nucleon. The shaded band corresponds to the assumed uncertainty on the inclusive cross section. There is an assumed 3\% normalization uncertainty in the DIS cross section (defined as $W>1.7 \mathrm{GeV}$ ), there is an additional $10 \%$ assumed normalization uncertainty in the QE and RES cross sections. . . . . . . . . . . . . . . 
5.8 From [71]. $v_{\mu}$ charged-current energy spectra measured in the six beam configurations of Table 5.1 and compared with the Monte Carlo prediction. Two Monte Carlo predictions are shown: one (thin line) with the ab initio calculation based on FLUKA05, the other (thick line) after constraining hadron production, focusing and detector parameters with the neutrino data. Panels along the bottom of each figure show the ratio of the measured and simulated spectra. . . . . . . . . . . . . . .

5.9 Figure From [71]. The error contribution to the predicted energy spectrum due to the current in the horn, the distribution of the horn current, offset in the horn, the scraping of the beam along the collimator baffle, and the counting of the protons on target as a function. . . . . . . . . . . . . . .

6.1 Input parameters for the kNN multivariate identification parameter. Red: the MC prediction which includes the beam tuning reweighting described in Section 5.2.1. Blue: the NC contamination. Black Points: the data recorded in the LE010/185 beam

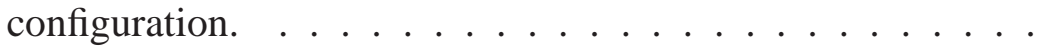

6.2 the $\mathrm{kNN}$ multivariate identification parameter for candidate $\mathrm{v}_{\mu^{-}}$ $\mathrm{CC}$ interactions. Red: the MC prediction which includes the beam tuning reweighting described in Section 5.2.1. Blue: the NC contamination. Black Points: the data recorded in the LE010/185 beam configuration. . . . . . . . . . . . . . . 98

6.3 The $\mathrm{v}_{\mu}$-CC energy spectrum of the MC (red), data (black), and QE-component (green) of the MC. The efficiency and NC contamination of interaction candidates selected by the multivariate identification parameter relative to the $\mathrm{CC}$ preselection are shown in blue with the y-axis on the right (also in blue). . . . . . . 
6.4 True kinetic energy of the leading proton in true QE events. The majority, $95 \%$, of the recoil have energy less than $1 \mathrm{GeV}$, nearly $99 \%$ have energy less than $2 \mathrm{GeV}$. . . . . . . . . . .

6.5 Event display of a characteristic true QE event in MINOS. On the top is the full event before any of the hit filters are applied. The plot on the bottom illustrates the grouping algorithm at work. In this case the line is drawn between the event vertex and the red hit that is in the candidate proton track furthest down stream from the event vertex. The black boxes represent the regions included in the grouping algorithm. Any hits whose hit centers are within one of the black boxes will be counted as part of the candidate proton track. . . . . . . . . . . . . . . .

6.6 Left: The opening angle between the MC true proton momentum and the reconstructed proton trajectory for all two track QE selected events. Right: The opening angle between the MC true proton momentum and the reconstructed proton trajectory for all MC true CCQE events with a candidate proton track. The "shoulder" near $60^{\circ}$ in the plot on the right is due to incomplete removal of the muon track. When there is more than 600 sigcor of pulse height near the event vertex, and the true proton is below detection threshold, it is possible for this algorithm to get confused and interpret the remaining muon hits as coming from a proton. This effect is illustrated in Figure 6.5. In the bottom panel of Figure 6.5 there are clearly two hits that were left over from the muon track. It is these hits that could potentially confuse this algorithm and create this shoulder. . . . . . . . . . . . .

6.7 The reconstructed hadronic shower energy of the neutrino event. The histograms show the dominant NEUGEN interaction types. The event is flagged as QE like if the the reconstructed hadronic shower energy is less than $0.25 \mathrm{GeV}$. . . . . . . . . 106 
6.8 Left: The fraction of true QE interactions in the given selection. Right: The efficiency of selecting true QE interactions relative to the number of true $\mathrm{QE}$ interactions in the $\mathrm{v}_{\mu}$-CC selection. The one track QE selection is in black, the two track QE is blue, and the two track background selection is red. . . . . . . . .

6.9 Left: The fraction of true resonance interactions in the given selection. Right: The efficiency of selecting true resonance interactions relative to the number of true resonance interactions in the $v_{\mu}$-CC selection. The one track QE selection is in black, the two track QE is blue, and the two track background selection is red.

6.10 Number hits in the candidate proton track. Top: Pre-selected $\left(\mathrm{v}_{\mu} \mathrm{CC}\right)$ events. Middle: Two track background selection. Bottom: Selected two track QE events. Left: Distributions with MC normalized to total data POT. Individual topological components of of the MC are shown as stacked histograms. Right: Ratio of data to MC

6.11 Summed pulse height of the scintillator strips in the candidate proton track. Top: Pre-selected $\left(v_{\mu} \mathrm{CC}\right)$ events. Middle: Two track background selection.Bottom: Selected two track QE events. Left: Distributions with MC normalized to total data POT. Individual topological components of of the MC are shown as stacked histograms. Right: Ratio of data to MC. . . . . . . . . . . . . 113

6.12 Total path length of the candidate proton track. Top: Pre-selected $\left(\mathrm{v}_{\mu} \mathrm{CC}\right)$ events. Middle: Two track background selection. Bottom: Selected two track QE events. Left: Distributions with MC normalized to total data POT. Individual topological components of of the MC are shown as stacked histograms. Right: Ratio of data to MC. . . . . . . . . . . . . . . 
6.13 Distance between maximum pulse height hit and furthest downstream hit. Top: Pre-selected $\left(\mathrm{v}_{\mu} \mathrm{CC}\right)$ events. Middle: Two track background selection. Bottom: Selected two track QE events. Left: Distributions with MC normalized to total data POT. Individual topological components of of the $\mathrm{MC}$ are shown as stacked histograms. Right: Ratio of data to MC. . . . . . . 115

6.14 Number of unused hits in the vertex hadronic shower. Top: Preselected $\left(v_{\mu} C C\right)$ events. Middle: Two track background selection. Bottom: Selected two track QE events. Left: Distributions with MC normalized to total data POT. Individual topological components of of the MC are shown as stacked histograms. Right: Ratio of data to MC. . . . . . . . . . . . . . . . . 116

6.15 Opening angle between the candidate proton track and the proton trajectory predicted from muon kinematics $\left(\Delta \theta_{p}\right)$. Top: Preselected $\left(\mathrm{v}_{\mu} \mathrm{CC}\right)$ events. Middle: Two track background selection. Bottom: Selected two track QE events. Left: Distributions with MC normalized to total data POT. Individual topological components of of the MC are shown as stacked histograms. Right: Ratio of data to MC. . . . . . . . . . . . . . . . . . . 117 
6.16 Summed pulse height in sigcor of all of the hits that are not used in either the muon track or the candidate proton track. Top: $\Delta \theta_{p}$ sideband selections (QE-like except $\Delta \theta_{p}>30$ ). Bottom: Selected two track QE events. Left: Distributions with MC normalized to total data POT. Individual topological components of of the MC are shown as stacked histograms. Right: Ratio of data to MC. The MC deficit in the 0-2000 sigcor region of the top plots when considered along side the MC deficit in the 60-90 degree region of Figure 6.15 is further evidence of a secondary nucleon from an SRC type interaction. The extra energy that we are seeing is substantial enough that additional quanta seems like a more likely explanation than a mis-modeling of detector effects

7.1 Comparison of the nominal MC to reweighted MC with $\pm 30 \%$ changes to the value of $M_{A}^{Q E}$. Only stopping muon events are included in this figure.

7.2 Comparison of the nominal MC to scaled MC with $\pm 2 \%$ scalings of the muon energy. Only stopping muon events are included in this figure. . . . . . . . . . . . . . .

7.3 Comparison of the nominal MC to reweighted MC with $\pm 15 \%$ changes to the value of $M_{A}^{R E S}$. Only stopping muon events are included in this figure. . . . . . . . . . . .

7.4 Comparison of the nominal MC to MC with a $+30 \%$ scale of the values of the Fermi momentum for protons and neutrons. Only stopping muon events are included in this figure. . . . . . .

7.5 Comparison of the nominal MC to MC with a $\pm 30 \%$ scale on the values of the Fermi momentum for protons and neutrons applied to the background like two track selection. Only stopping muon events are included in this figure. . . . . . . . . . . 
7.6 Starting from the top and moving down are the $Q^{2}$ distributions for the low hadronic shower energy sub-sample, the 2 track QElike sub-sample, and the 2 track background-like sub-sample. On the left are the actual $Q^{2}$ distributions using a binning that is much finer than the binning actually used in the fits. On the right are data/MC plots using the actual fit binning. The blue histograms show the dominant topology for each of the three selections. . . . . . . . . . . . . . .

7.7 The $1-\sigma\left(\right.$ defined as $\left.\Delta \chi^{2}=1\right)$ contours for the 1 track NuInt09 analysis and this mock data analysis. The contour for $M_{A}^{Q E}$ and the $E_{\mu}-$ scale is shown on the left. The contour for $M_{A}^{Q E}$ and $k_{\text {Fermi }}^{Q E}-$ scale is shown on the right. The one track NuInt09 result contour is shown in red. The contours from this mock data analysis are shown in blue. The three selection analysis significantly improves the size of the allowed parameter space. In addition the additional selections break the correlation between $M_{A}^{Q E}$ and the

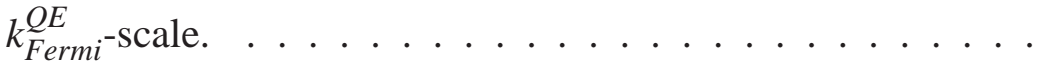

7.8 Best fit $Q_{Q E}^{2}$ distributions for the fit to data that matches the mock data procedure. Top: One track QE-like selection. Middle: Two track QE-like selection. Bottom: Two track resonance-like selection. Left: Finely binned $Q_{Q E}^{2}$ distribution. Red: Best fit $Q_{Q E}^{2}$ distribution. Blue: Nominal $Q_{Q E}^{2}$ distribution. Black: Best fit $Q_{Q E}^{2}$ distribution of the dominant interaction type of each selection. Right: Data/MC ratio binned using the same binning as the

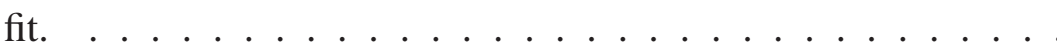

7.9 Left: true resonance $Q_{Q E}^{2}$ distributions. Blue: distribution for $k_{F e r m i}^{R e s}$ equal to 2.87. Red: nominal true resonance $Q_{Q E}^{2}$ distribution. Right: the $k_{F e r m i}^{R e s}$ equal to 2.87 distribution divided by the nominal distribution. . . . . . . . . . . . . . 
7.10 Red: suppression function from [88] with error band that includes both statistical and systematic uncertainty. Blue: suppression function from a $k_{\text {Fermi }}^{\text {Res }}$ value of 2.87. . . . . . . . . .

7.11 Best fit $Q_{Q E}^{2}$ distributions for the fit to data that uses the low $Q^{2}$ suppression function from [88]. Top: One track QE-like selection. Middle: Two track QE-like selection. Bottom: Two track resonance-like selection. Left: Finely binned $Q_{Q E}^{2}$ distribution. Red: Best fit $Q_{Q E}^{2}$ distribution. Blue: Nominal $Q_{Q E}^{2}$ distribution. Black: Best fit $Q_{Q E}^{2}$ distribution of the dominant interaction type of each selection. Right: Data/MC ratio binned using the same binning as the fit. . . . . . . . . . . .

7.12 One sigma contours for all the different pairs of fit parameters. For each plot the parameters not shown are marginalized. Black: three sample combined fit. Red: one track QE only fit. Blue: two track QE only fit. Violet: two track resonance only fit. Green: one and two track QE combined fit. Starting with the top left:

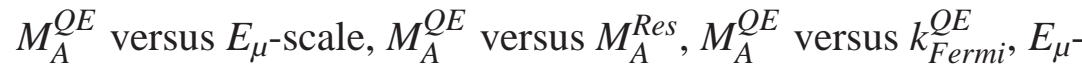
scale versus $M_{A}^{\text {Res }}, E_{\mu}$-scale versus $k_{F e r m i}^{Q E}, M_{A}^{\text {Res }}$ versus $k_{F e r m i}^{Q E}$. .

7.13 The background correction function for the primary fit using the procedure outlined in Section 7.5.1. The correction function is calculated from by fitting the ratio of the data to the $\mathrm{MC}$ from the two track background like sub-sample. This data is fit to a third order polynomial which is the green line shown in the Figure. .

7.14 Best fit $Q_{Q E}^{2}$ distributions for the fit to data using the procedure outlined in Section 7.5.1. Top: One track QE-like selection. Bottom: Two track QE-like selection. Red: Best fit $Q_{Q E}^{2}$ distribution. Blue: Nominal $Q_{Q E}^{2}$ distribution. Black: Best fit $Q_{Q E}^{2}$ distribution of the true QE composition of the sub-sample. Right: Data/MC ratio binned using the same binning as the fit. . . . 
7.15 Best fit values (along with the HESSE) errors for different definitions of the low hadronic energy sub-sample. . . . . . . . . 142

7.16 Best fit values (along with the HESSE errors) for different definitions of the $\Delta \theta_{p}$ dividing line between the two track QE-like and two track background like sub-samples. . . . . . . . . . . 143

7.17 Best fit values (along with the HESSE errors) for different definitions of the unused shower strip hit dividing line between the two track QE-like and two track background like sub-samples.

7.18 Best fit values (along with the HESSE errors) for different definitions of the unused shower strip hit dividing line between the two track background like and the sub-sample of interaction candidates that are not used in this analysis. . . . . . . . . . . 145

7.19 Best fit values (along with the HESSE errors) for the horn position systematic. . . . . . . . . . . . . . 146

7.20 Best fit values (along with the HESSE errors) for the baffle scrapping systematic. . . . . . . . . . . . . . 146

7.21 Best fit values (along with the HESSE errors) for the horn current calibration systematic. . . . . . . . . . . . . . . 147

7.22 Best fit values (along with the HESSE errors) for the horn current distribution systematic. . . . . . . . . . . . . 147

7.23 Best fit values (along with the HESSE errors) for the target $z$ position along with hadron production systematics. . . . . . 148

7.24 Best fit values (along with the HESSE errors) for the DIS model systematic. . . . . . . . . . . . . . . 149

7.25 Best fit values (along with the HESSE errors) due to the low $Q^{2}$ resonance suppression function. . . . . . . . . . . 149

7.26 Best fit values (along with the HESSE errors) for some of the intranuclear rescattering uncertainties. . . . . . . . . 150

7.27 Best fit values (along with the HESSE errors) for some of the intranuclear rescattering uncertainties. . . . . . . . 
7.28 Left: the ratio of data to $\mathrm{MC}$ for the nominal $\mathrm{MC}$ along with the $\pm 1 \sigma$ shifts to the nucleon knockout and nucleon cross section intranuclear rescattering systematics applied to the nominal MC. Right: the ratio of the nominal MC to the $\pm 1 \sigma$ nucleon knockout and nucleon cross section intranuclear rescattering systematics applied to the nominal MC. Above: the low hadronic energy QElike sub-sample. Below: the two track QE-like sub-sample. . .

$7.29 \chi^{2}$ scan of the nucleon cross section INTRANUKE systematic parameter. The $\pm 1 \sigma$ uncertainty due to the nucleon cross section parameter is defined as the fit parameter shift due to a $\Delta \chi^{2}$ from the minimum of $1 . \ldots \ldots \ldots \ldots \ldots$

$7.30 \chi^{2}$ scan of the nucleon knockout INTRANUKE systematic parameter. The $\pm 1 \sigma$ uncertainty due to the nucleon cross section parameter is defined as the fit parameter shift due to a $\Delta \chi^{2}$ from the minimum of $1 . \ldots \ldots \ldots$

7.31 Best fit values (along with the HESSE errors) for some of the intranuclear rescattering uncertainties. . . . . . . . .

7.32 One sigma contour of the $M_{A}^{Q E}$ (a scale factor on the nominal value of $M_{A}^{Q E}$ equal to $0.99 \mathrm{GeV}$ ) parameter versus the hadronic energy offset systematic parameter. The hadronic energy offset parameter shows very little correlation with any of the other fit parameters. Thus a plot of any other fit parameter versus the hadronic energy offset will look very similar to this one. . . .

7.33 One sigma contour of the $M_{A}^{Q E}$ parameter versus the $M_{A}^{R E S}$ parameter. Both of these parameters are scale factors on the nominal values of these parameters. The nominal value for $M_{A}^{Q E}$ is $0.99 \mathrm{GeV}$ while the nominal value for $M_{A}^{R E S}$ is $1.12 \mathrm{GeV}$. The use of the two track QE-like and the two track background like subsamples breaks the correlations between the both of the axialvector parameters. . . . . . . . . . . . . 
7.34 One sigma contour of the $M_{A}^{Q E}$ (a scale factor on the nominal value of $M_{A}^{Q E}$ equal to $0.99 \mathrm{GeV}$ ) parameter versus the $k_{F e r m i}^{Q E}$ parameter. The tension between the low hadronic energy offset sub-sample and the two track QE-like sub-sample largely breaks the correlation between these parameters. There is still some correlation in the lower allowed region of the quasi-elasti axialvector mass parameter. . . . . . . . . . . . .

7.35 One sigma contour of the $M_{A}^{Q E}$ (a scale factor on the nominal value of $M_{A}^{Q E}$ equal to $0.99 \mathrm{GeV}$ ) versus the $E_{\mu}$-scale systematic parameter. There is a strong correlation between these parameters due to the muon energy dependence of $Q_{Q E}^{2}$. . . . . .

8.1 Measured $M_{A}^{Q E}$ values as a function of the neutrino target nucleon number. Previous measurements are red, while the measurement presented in here is blue. . . . . . . . . . . .

8.2 Left: $Q^{2}$ distributions used in this analysis along with an alternative to the low hadronic energy sub-sample called the 'one track' sub-sample. Right: the same distributions as the left but on a semi-log scale. . . . . . . . . . . . . . 163 


\section{List of Tables}

2.1 The possible forms of the weak interaction that are allowed in Dirac's theory . . . . . . . . . . . . . . . .

2.2 Lorentz invariant kinematic variables that describe the charged current neutrino-nucleon scattering. The mass $M$ is the mass of the struck nucleon. . . . . . . . . . . . . . .

3.1 Summary of some $M_{A}^{Q E}$ measurements. Table reproduced from $[52] . \ldots \ldots \ldots \ldots \ldots \ldots . \ldots \ldots$

3.2 Best fit results for $M_{A}^{Q E}$ using the likelihood functions given in Equations 3.1 to 3.4 as discussed in [42]. . . . . . . . . . .

5.1 The target position refers to the distance the target was displaced upstream of its default position inside the rst focusing horn. The peak (i.e., most probable) neutrino energy $E_{v}$ is determined after multiplying the muon-neutrino ux predicted by the beam Monte Carlo simulation by charged-current cross-section. The r.m.s. refers to the root mean square of the peak of the neutrino energy distribution. The $0 \mathrm{kA}$ "horn-off" beam is unfocused and has a broad energy distribution. . . . . . . . . . . .

7.1 Best fit results and for the two fit configurations and reduced $\chi^{2}$ values according to 7.1 for the best fit MC. . . . . . . . . . . 127

7.2 Best fit values for the fit to data. The errors shown are the MINUIT returned HESSE errors. . . . . . . . . . . . . . . . . 130 
7.3 Best fit values for the fit to data using the suppression function from [88]. The errors shown are the MINUIT returned HESSE errors. . . . . . . . . . . . . . . . .

7.4 Best fit values for the baseline first and second fit iteration using the procedure outlined in Section 7.5.1. No further iterations were necessary because there was very little change in the background correction function. The errors shown are the HESSE errors from the MINUIT software package. . . . . . . . . . . . . . . 140

7.5 Contribution to the systematic error due to the different categories defined in this Section on the $M_{A}^{Q E}$ fit parameter. . . . . . . . . 154

7.6 Best fit values for the along with contributions to the uncertainty due to the fit and the systematics considered in Section 7.5.4 . . 156 


\section{Chapter 1}

\section{Introduction}

\subsection{Introduction}

Particle physicists rely on the The Standard Model for the theoretical framework to describe the interactions of the fundamental particles and forces of the universe. The Standard Model is one of the most throughly tested and successful theories of science. However, in the last thirty years, developments within the study of neutrinos have revealed a weakness in the Standard Model. This is the apparent flavor-oscillation within the neutrino sector, exemplified by the so-called Atmospheric Neutrino Anomaly, and the Solar Neutrino Problem.

The Standard Model describes twelve fundamental fermionic particles divided into two separate families: the leptons and the quarks of the hadronic sector. Neutrinos belong to the lepton family of which there are three flavors: the electron, muon and tau neutrinos $\left(\mathrm{v}_{e}, \mathrm{v}_{\mu}\right.$, and $\left.\mathrm{v}_{\tau}\right)$. The Standard Model describes the neu-

trino as massless, chargeless spin- $\frac{1}{2}$ particles that also carry no color charge and only interact via the weak nuclear force. Wolfgang Pauli originally proposed the existence of the neutrino to solve the problem of apparent momentum nonconservation in nuclear $\beta$-decay [1]. The classical two body $\beta$-decay is $n \rightarrow p+e$, under this interaction if energy and momentum are conserved the electron should be emitted with discrete energy. However the observed energy of the electron 
from $\beta$-decay was a continuous spectrum. The only way the continuous spectrum of the electron can be explained without violating momentum conservation was by postulating the existence of an additional particle, nearly impossible to detect, that carried away a fraction of the total energy. This brilliant though controversial idea resolved the conflict between momentum conservation and the observed electron spectrum. It took 26 years to finally confirm Pauli's hypothesis, when Reines and Cowen directly observed a neutrino interaction through the inverse $\beta$-decay mechanism at the Savannah River nuclear reactor [?].

The Solar Neutrino Problem is a discrepancy between the number of neutrinos interacting in the Earth (and the experiments of physicists) and the expected number of neutrinos predicted by models of the rates of nuclear reactions within the sun. The Sun releases energy through nuclear fusion, primarily through the proton-proton chain. The proton-proton chain converts four hydrogen nuclei into one helium nucleus, two neutrinos, two positrons and some excess energy. The Davis and Bahcall experiment at the Homestake mine was the first to measure the solar neutrino flux. They measured a deficit compared to theoretical predictions of the solar model [3][4][5].

The Atmospheric Neutrino Anomaly is a discrepancy in the flux of muon neutrinos relative to the flux of electron neutrinos due to cosmic ray interactions in the upper atmosphere. Cosmic rays incident on the upper atmosphere interact with nucleons that lie within the constituent molecules of the atmosphere, producing a shower of secondary and tertiary particles which include large numbers of pions. The pions eventually decay to muons which will decay to electrons by the following processes:

$$
\begin{gathered}
\pi^{-} \rightarrow \mu^{+}+v_{\mu} \\
\mu^{-} \rightarrow e^{+}+v_{e}+v_{\mu}
\end{gathered}
$$

From the very well measured branching ratios of pions, equations 1.1 and $1.2 \mathrm{im}-$ ply that the ratio of $v_{\mu}\left(\bar{v}_{\mu}\right): v_{e}\left(\bar{v}_{e}\right)$ arriving should be $2: 1$, however experimental measurements of this ratio have shown a deficit in the number of $v_{\mu}$ [6] [7] [8]. 
The solution to both the Solar Neutrino Problem and the Atmospheric Neutrino Anomaly was first suggested by Bruno Pontecorvo. In 1967 [9] Pontecorvo proposed that neutrinos were oscillating between the neutrino's creation and the neutrinos detection. Neutrino oscillation is a mixing of the neutrino flavor that is analogous to flavor changing weak decays in the quark sector. Pontecorvo suggested that if neutrinos had finite mass and if the weak eigenstates of the neutrino (the interaction state of the neutrino) were not the same as the mass eigenstates of the neutrino (the propagation state of the neutrino), then a mixing matrix could be formed in a procedure similar to the Cabbibo-Kobayashi-Maskawa (CKM) matrix of the quarks. Thus a neutrino created in one flavor eigenstate would became a superposition of all the flavor eigenstates as the neutrinos propagate in the mass eigenstates. This enables the detection of a different flavor eigenstate at some later time. This phenomenon can be used to explain both the Solar Neutrino Problem and the Atmospheric Neutrino Anomaly, though it requires massive neutrinos and a theory beyond the Standard Model.

Neutrinos have been studied from cosmic ray production, and from fission decay within nuclear reactors. However there are limitations to this approaches. These limitations are primarily due to having limited control over the conditions of the neutrino's creation. The approach of more recent experiments, greater control over the production conditions of the neutrino, is to use a beam of neutrinos produced from particle accelerators specialized in the production of neutrino beams. This is the source for neutrinos used in the Main Injector Neutrino Oscillation Search (MINOS) experiment, currently running at the Fermi National Accelerator Laboratory (FNAL) in Batavia, Illinois.

The physics goal of the MINOS experiment is to make a precise measurement of the parameters that govern muon disappearance $\left(v_{\mu}+v_{\mu}\right)$. MINOS has been very successful at this, publishing the world's most precise measurement of the atmospheric mass splitting $\Delta m_{23}^{2}$ [10]. The MINOS experiment uses the neutrino beam produced by the Neutrinos at the Main Injector (NuMI) facility. This beam is greater than $95 \%$ pure muon neutrino. MINOS measures the composition and 
spectrum of the neutrino beam by sampling the beam at two locations using large iron and scintillator tracking/sampling colorimeters; the Near Detector at FNAL and the Far Detector at the Soudan Underground Laboratory, $734 \mathrm{~km}$ away in Soudan Minnesota. MINOS maximizes their sensitivity to the neutrino oscillation parameters by comparing the spectrum at the Near Detector, where the neutrinos have not traveled far enough to have oscillated, to the spectrum at the Far Detector where the neutrinos will have oscillated (if the oscillations are true).

\subsection{Neutrino Oscillation Theory}

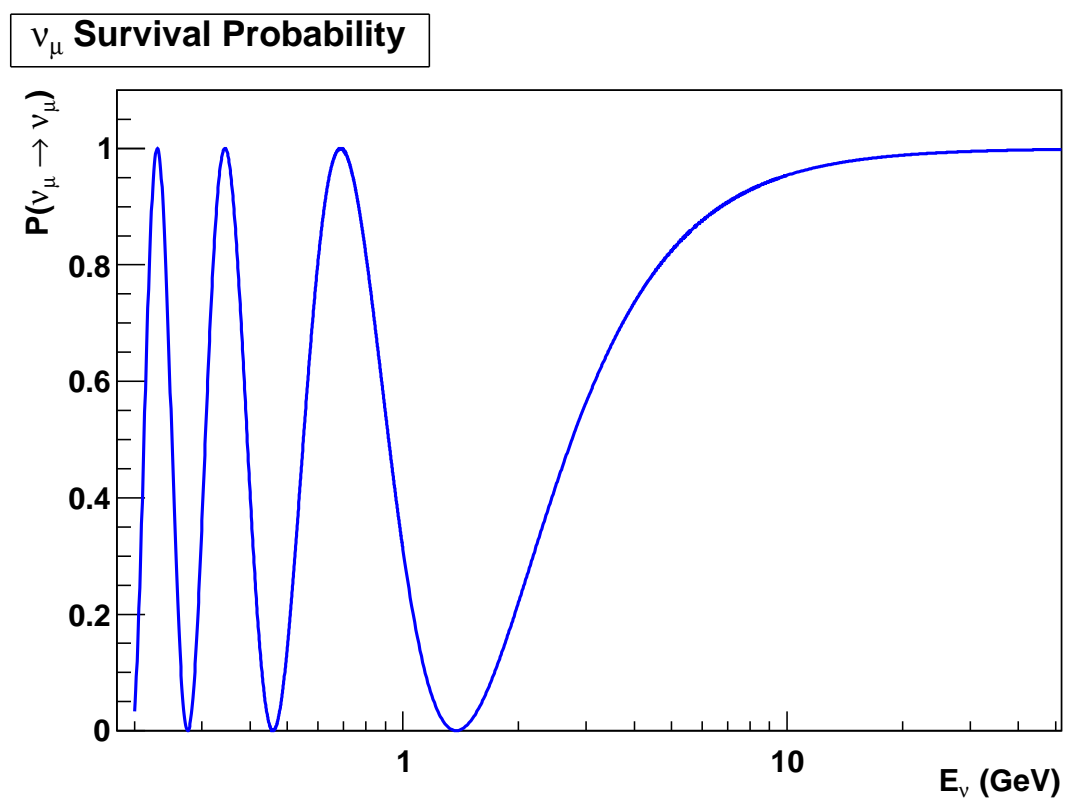

Figure 1.1: Survival probability of muon neutrinos as a function of energy given the MINOS baseline and best fit values for the oscillation parameters. The first minimum from the right is the dip that MINOS is most sensitive to.

Neutrino oscillations occur outside of the Standard Model. This is because a necessary condition for neutrino oscillations is massive neutrinos and neutrinos 
have no mass within the Standard Model. Thus neutrinos oscillations are evidence of "beyond the Standard Model" physics. The probability that a muon neutrino of a given energy will survive with out oscillating after some distance is given by the survival probability given by:

$$
P\left(\mathrm{v}_{\beta} \rightarrow \mathrm{v}_{\beta}(L / E)\right)=1-\sin ^{2} 2 \theta \sin ^{2}\left[1.27 \Delta m^{2}(L / E)\right]
$$

Equation 1.3 demonstrates that for an experiment that has a fixed baseline (L) the strength of the oscillation of a neutrino of a given energy $E$ is a function of only the mixing angle $\theta$ and the mass splitting $\Delta m^{2}$. The survival probability for $v_{\mu}$ as function of energy as given by equation 1.3 is shown in Figure 1.1. This plot assumes the MINOS baseline of $734 \mathrm{~km}, \sin ^{2} 2 \theta=1$ and $\Delta m^{2}=2.32 \times$ $10^{-3} \mathrm{GeV}^{2}$.

\subsection{Neutrino Oscillations in MINOS}

MINOS looks for an energy dependent disappearance of muon neutrinos at the Far Detector as compared to the no oscillations expectation. MINOS uses the data taken at the Near Detector (ND) to validate the Monte Carlo (MC) simulation of the neutrino interactions. MINOS then extrapolates the neutrino energy spectrum at the ND, as predicted by the simulation, to the Far Detector. This prediction assumes that the muon neutrinos are not oscillating into other neutrino flavors. Deviations between the extrapolated Far Detector spectrum (from the ND) and the data taken at the Far Detector are then fit to extract the neutrino oscillation parameters; $\Delta m^{2}$ and the mixing angle $\theta$.

Figure 1.2 shows the reconstructed neutrino energy spectrum of neutrino events in the MINOS Far Detector, as well as the predicted un-oscillated energy spectrum as extrapolated from the ND, and the best fit predicted energy spectrum from the oscillation assumption. Figure 1.2 also shows the ratio of the data to the $\mathrm{MC}$ no oscillation prediction along with best fit predictions from three different theoretical models, oscillations, neutrino decay, and neutrino decoherence. The data to 


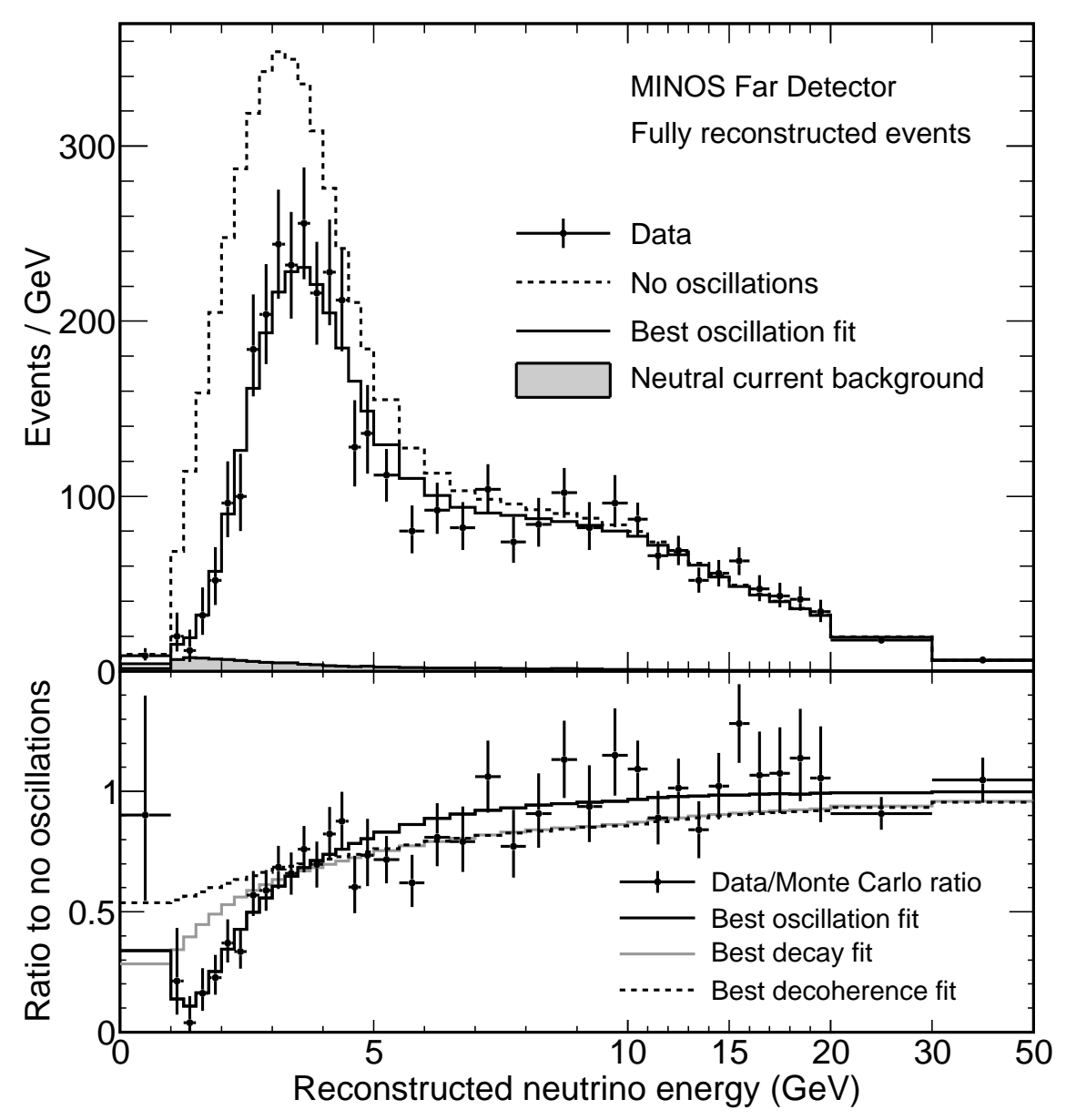

Figure 1.2: From [10]. Top: The MINOS energy spectra of fully reconstructed events in the Far Detector classified as charged current interactions. The dashed histogram represents the spectrum predicted from measurements in the Near Detector assuming no oscillations, while the solid histogram reflects the best fit of the oscillation hypothesis. The shaded area shows the predicted neutral current background. Bottom: The points with error bars are the background-subtracted ratios of data to the no-oscillation hypothesis. Lines show the best fits for: oscillations, decay [11], and decoherence [12]. 


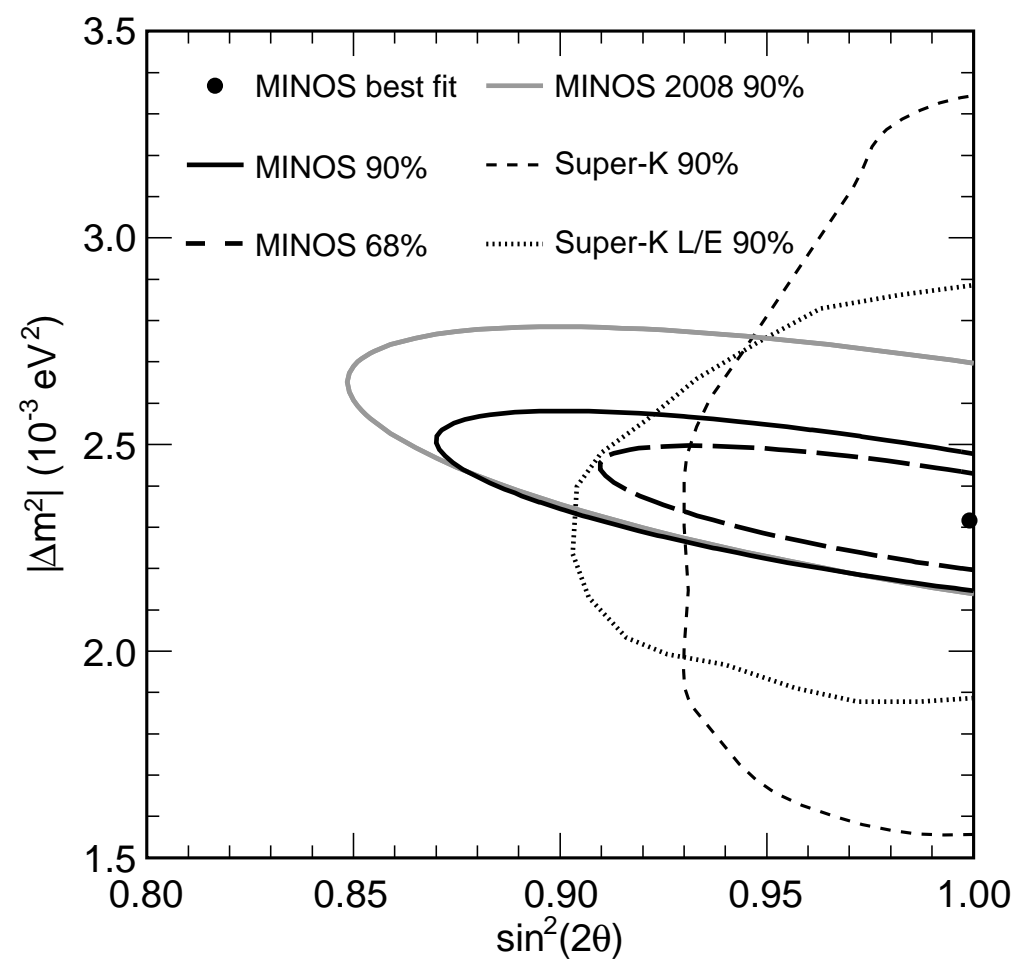

Figure 1.3: From [10]. Likelihood contours of $68 \%$ and 90\% C.L. around the best fit values for the mass splitting and mixing angle. Also shown are contours from previous measurements [13, 14].

MC ratio shows the characteristic 'dip' structure that indicates the presence of neutrino oscillations. MINOS excludes the neutrino decay hypothesis at seven standard deviations, and the neutrino decoherence hypothesis at nine standard deviations.

Figure 1.3 shows the best fit neutrino oscillation parameters extracted from the oscillation fit to the MINOS Far Detector, along with the $68 \%$ and $90 \%$ confidence 
intervals for the measurement of the neutrino oscillation parameters. Figure 1.3 also shows the $90 \%$ confidence interval from the MINOS 2008 oscillation analysis [14] and the $90 \%$ confidence interval from two separate Super-K neutrino oscillation analyses.

\subsection{Motivation for Neutrino Quasi-Elastic Measure- ments}

MINOS's neutrino oscillation analysis is reliant on knowledge of the event rate in the Near and Far Detectors. Event rate is a convolution of neutrino flux, neutrino cross-section, and the number of interaction targets within the neutrino beam. Neutrino interaction cross sections are not well known for lower neutrino energies $\left(E_{v}<10 \mathrm{GeV}\right)$ with cross section uncertainties for certain exclusive final states, such as quasi-elastic scattering, at the 20\%-30\% level.

Neutrinos provide a unique probe of the internal structure of the nucleus and nucleon due to the neutrino's singular coupling to the weak force. This makes neutrinos (along with parity violating electron scattering which probe the strange component of nucleons) the only viable probes for examining the weak-charge distribution of the nucleon and the weak force dependence of the nuclear structure. Differences have been observed between older measurements of charged current quasi-elastic interactions that were statistics limited and performed on deuterium, and more recent experiments that have had orders of magnitude more neutrino interactions and have been performed on higher $\mathrm{Z}$ nuclear targets. The current inclinations of the neutrino interaction community is that this discrepancy is due to the presence of neutrino interactions on multi-nucleons, such as short range nuclear correlations (SRC) and meson exchange currents (MEC). These nucleonnucleon interactions have been observed with charged leptons but never with neutrinos. Because they have never been observed in neutrino interactions it's not completely clear how to properly simulate these interactions in neutrino MC, thus they are unsimulated within the present generation of neutrino event generators. 


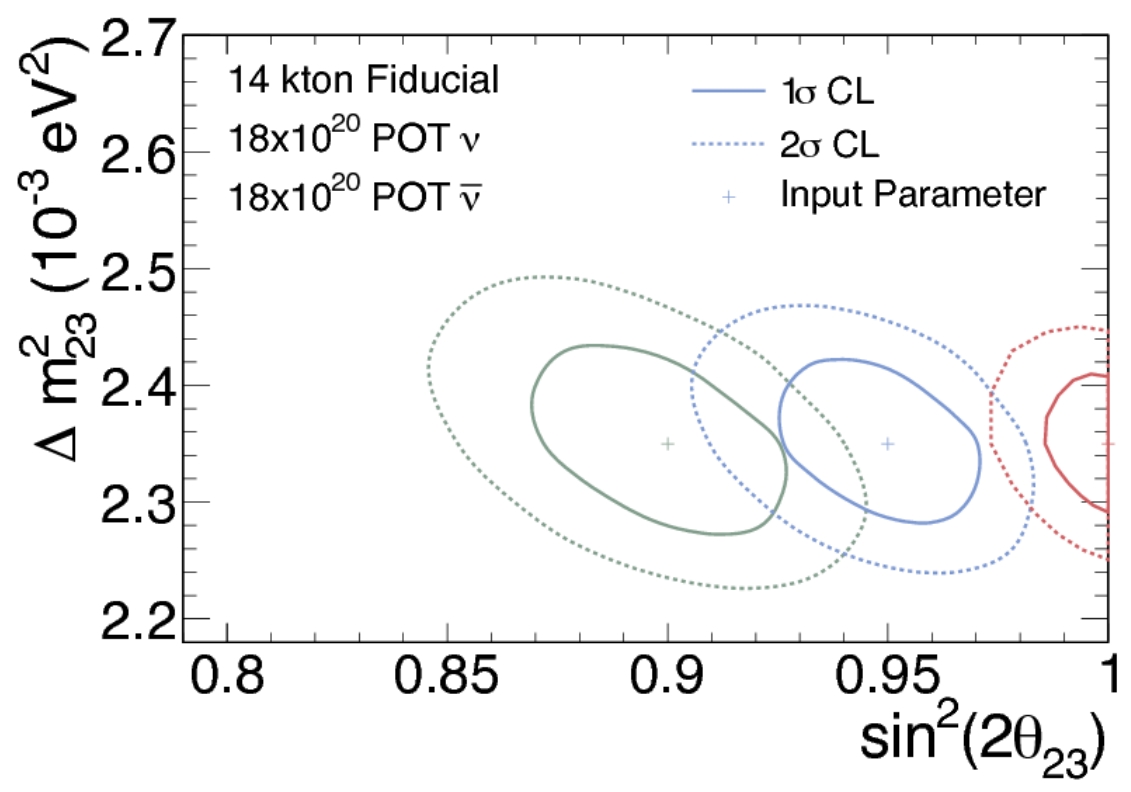

Figure 1.4: The expected 1 and $2 \sigma$ measurements of $\sin ^{2}\left(2 \theta_{23}\right)$ for 6 years of NOvA running ( 3 yrs in neutrino mode +3 yrs in antineutrino mode) using numu quasi elastic events in NOvA. The input $\Delta m^{2}$ is taken to coincide with recent MINOS measurements and the three choices of mixing angle are made consistent with the data from Super-Kamiokande ( $>0.92$ at $90 \%$ confidence limit).

An improved understanding of exclusive final state neutrino cross sections is also important for the next generation of neutrino experiments, such as NOvA and T2K. These experiments were conceived to measure a different mixing angle, $\theta_{13}$, which is the mixing angle responsible for the $v_{\mu} \rightarrow v_{e}$ sub-dominant oscillations. These experiments (particularly NOvA) are also capable of making very accurate measurements of the $\theta_{23}$ mixing angle and, should the difference $1-\sin ^{2}\left(2 \theta_{23}\right)$ be large enough, rule out maximal mixing (the complete disappearance of all the neutrinos of a particular energy).

Figure 1.4 shows the expected NOvA measurements of the $v_{\mu} \nrightarrow v_{\mu}$ oscillations using charged current quasi-elastic (CCQE) events. NOvA will use CCQE events 
because NOvA will have much better statistics than MINOS did, and using exclusively CCQE events minimizes the effect of energy resolution smearing. However because the current generation of neutrino generators do not contain SRCs or MECs, and the signature of these multi-nucleon interactions is the presence of additional low energy particles in the final state, it is possible that these potentially below detection threshold low energy particles may introduce a significant bias into the measurement of the oscillation parameters. Thus, in order to be fully confident in the NOvA measurement of the $v_{\mu}+v_{\mu}$ oscillations, it is necessary to have a full understanding of the impact of the multi-nucleon interactions on the CCQE cross section. 


\section{Chapter 2}

\section{Theory of the Weak Interaction}

\subsection{Weak Interaction Phenomenology}

\subsubsection{Fermi's Point-like Four-Fermion Theory of $\beta$-Decay}

It took four years from when Wolfgang Pauli first proposed the existence of the neutrino with his "Dear Radioactive Ladies and Gentlemen" letter [1] for a full quantum field theory (QFT) of the weak interaction to be developed. The first QFT of the weak interaction, proposed by Enrico Fermi, considered the electronneutrino pair emitted in the neutron to proton nuclear transition $(n \rightarrow p+e+$ $\bar{v})$ to be analagous to the emission of photons in nuclear $\gamma$-decay. Inspired by quantum electrodynamics (QED), Fermi treated the interaction as happening at one spacetime point. The interaction involved a 4-vector weak current between the neutron and the proton. In addition, to ensure that the interaction would be Lorentz invariant, Fermi included an additional current between the electron and the neutrino, finally Fermi constructed a 'current-current' interaction amplitude:

$$
\frac{G_{F}}{\sqrt{2}} \bar{u}_{p} \gamma^{\mu} u_{n} u_{e^{-}} \gamma_{\mu} u_{\bar{v}}=\frac{G_{F}}{\sqrt{2}} j_{N}^{\mu} j_{l}^{\mu}
$$


In Equation $2.1 G_{F}$ is Fermi's constant, equal to $1.166 \times 10^{-5} \mathrm{GeV}^{-2}, u$ and $\bar{u}$ are Dirac spinors, $\gamma_{\mu}$ and $\gamma^{\mu}$ are Dirac matrices, $\mu$ is the four component label for spatial and and time dimensions, $j_{N}^{\mu}$ is the nucleon current, and $j_{l}^{\mu}$ is the leptonic current. In Dirac's representation, $\gamma^{\mu}$ are matrices defined as:

$$
\begin{aligned}
& \gamma^{0}=\left(\begin{array}{cc}
\mathbf{I}_{2} & \mathbf{0} \\
\mathbf{0} & -\mathbf{I}_{2}
\end{array}\right) \quad \gamma^{1}=\left(\begin{array}{cc}
\mathbf{0} & \boldsymbol{\sigma}_{1} \\
-\boldsymbol{\sigma}_{1} & 0
\end{array}\right) \\
& \gamma^{2}=\left(\begin{array}{cc}
\mathbf{0} & \boldsymbol{\sigma}_{2} \\
-\boldsymbol{\sigma}_{2} & 0
\end{array}\right) \quad \gamma^{3}=\left(\begin{array}{cc}
\mathbf{0} & \boldsymbol{\sigma}_{3} \\
-\boldsymbol{\sigma}_{3} & 0
\end{array}\right)
\end{aligned}
$$

where $\mathbf{I}_{\mathbf{2}}$ is the $2 \times 2$ unit matrix, $\mathbf{0}$ is the $2 \times 2$ zero matrix, and $\boldsymbol{\sigma}_{i}$ are the Pauli spin matrices. Equation 2.1 can be rewritten in QFT notation, with $\psi(\mathbf{x}, t)$ denoting a quantum field, as a local interaction density:

$$
\frac{G_{F}}{\sqrt{2}} \overline{\hat{\psi}}_{p}(\mathbf{x}, t) \gamma^{\mu} \hat{\psi}_{n}(\mathbf{x}, t) \overline{\hat{\psi}}_{e^{-}}(\mathbf{x}, t) \gamma_{\mu} \hat{\psi}_{\bar{v}}(\mathbf{x}, t)
$$

The current-current formalism pioneered by Fermi was a success at describing the characteristics of $\beta$-decay, except for nucleon spin flip transitions (transitions where the nuclear spin changes by one). The next insight into the weak interaction came two years later when George Gamow and Edward Teller proposed a more general form of the four-fermion interaction. Gamow and Teller's breakthrough consisted of allowing for the experimentally observed nucleon spin flips by introducing a two index term as shown in Equation 2.4.

$$
\bar{u}_{p} \sigma_{\mu v} u_{n} \quad \text { where } \quad \sigma_{\mu v} \frac{i}{2}\left(\gamma_{\mu} \gamma_{v}-\gamma_{v} \gamma_{\mu}\right)
$$

The next two decades saw a wealth of new discoveries within the realm of particle physics: the neutrino was directly observed for the first time, both the muon and pion were discovered, and many new observations of weak interactions were made. These observations which could not be explained by the previously 
proposed theoretical forms of the weak interaction. The solution, first proposed by Lee and Yang [15], called into doubt the conservation of parity (mirror symmetry) in weak interactions. Much later it was determined that the weak interactions maximally violates parity conservation, but this idea was very radical at the time.

\subsubsection{The Axial Vector Structure of the Weak Interaction}

Parity is an inversion of spatial coordinates, like a reflection in a mirror. Parity allows for several definitions of physical quantities, polar vectors, axial vectors, scalars, and pseudoscalars. A polar vector, $\mathbf{V}$, is a vector that transforms in the same way as the coordinate $\mathbf{x}$ under the parity operator, $\mathbf{P}$ :

$$
\mathbf{P}: \mathbf{x} \rightarrow-\mathbf{x} \quad, \quad \mathbf{P}: \mathbf{V} \rightarrow-\mathbf{V}
$$

polar vector examples from introductory physics are quantities such as velocity, momentum, and electric current. Axial vectors transform in the same was as the cross product of two polar vectors. There is no sign change under the parity operator of axial vectors as shown in Equation 2.6.

$$
\mathbf{P}: \mathbf{U} \times \mathbf{V} \rightarrow(-\mathbf{U}) \times(-\mathbf{V})=\mathbf{U} \times \mathbf{V} \quad, \quad \mathbf{P}: \mathbf{A} \rightarrow \mathbf{A}
$$

angular momentum, and spin are both examples of axial vectors. Scalars do not change sign under the parity operator, this can be demonstrated by performing the parity operator on the dot product of two polar vectors as shown in Equation 2.7.

$$
\mathbf{P}: \mathbf{U} \cdot \mathbf{V} \rightarrow(-\mathbf{U}) \cdot(-\mathbf{V})=\mathbf{U} \cdot \mathbf{V}
$$

Pseudoscalars, however do change sign under the parity operator. Pseudoscalars can be formed from the triple scalar product of three polar vectors as shown in Equation 2.8.

$$
\mathbf{P}: \mathbf{U} \cdot(\mathbf{V} \times \mathbf{W}) \rightarrow(-\mathbf{U}) \cdot(\mathbf{V} \times \mathbf{W})
$$


It can be shown that the free particle solution to the Dirac equation of definite parity is:

$$
\psi(\mathbf{x}, t)=N\left(\begin{array}{c}
\phi \\
\frac{\mathbf{\sigma} \cdot \mathbf{p}}{E+m} \phi
\end{array}\right) e^{-i E t+i \mathbf{p} \cdot \mathbf{x}}
$$

where $\phi$ is a two component Dirac spinor and $N$ is a normalisation factor. Equation 2.9 transforms thusly under the parity operator:

$$
\mathbf{P}: \psi(\mathbf{x}, t) \rightarrow \psi_{P}(\mathbf{x}, t)=\gamma^{0} \psi(-\mathbf{x}, t)
$$

Thus a unitary quantum field operator, $\hat{\mathbf{P}}$ can be defined such that:

$$
\psi_{P}(\mathbf{x}, t)=\hat{\mathbf{P}} \psi(\mathbf{x}, t) \hat{\mathbf{P}}^{-1}=\gamma^{0} \psi(-\mathbf{x}, t)
$$

Using Equation 2.11 it becomes possible to consider the effects of the parity operator on the previous forms of the weak interaction. As an example consider the spatial part of Fermi's weak 4-vector current:

$$
\begin{aligned}
\hat{\bar{\psi}}_{1 \mathbf{P}}(\mathbf{x}, t) \gamma^{\mu} \hat{\psi}_{2 \mathbf{P}}(\mathbf{x}, t) & =\hat{\psi}_{1 \mathbf{P}}^{\dagger}(\mathbf{x}, t) \gamma^{0} \gamma^{\mu} \hat{\psi}_{2 \mathbf{P}}(\mathbf{x}, t) \\
& =\hat{\psi}_{1}^{\dagger}(-\mathbf{x}, t) \gamma^{0} \gamma^{0} \gamma^{\mu} \gamma^{0} \hat{\psi}_{2}(-\mathbf{x}, t) \\
& =-\hat{\psi}_{1}(-\mathbf{x}, t) \gamma^{\mu} \hat{\psi}_{2}(-\mathbf{x}, t)
\end{aligned}
$$

$$
\text { where } \gamma^{0} \gamma^{\mu}=-\gamma^{\mu} \gamma 0 \text { and }\left(\gamma^{0}\right)^{2}=1
$$

Equation 2.12 shows that the spatial components of the 4-vector current transform as a polar vector under the parity operator. Similarly the time component of Fermi's 4-vector current transforms as a scalar under the parity operator. Thus the 4-vector current of Fermi's initial theory of the weak interaction does not allow for the violation of parity. An extension of the theory must be made to allow for parity violation. Parity violation can be accommodated by introducing terms that 
transform as axial vectors. This is done using the $\gamma^{5}$ matrix defined as:

$$
\gamma^{5}=i \gamma^{0} \gamma^{1} \gamma^{2} \gamma^{3} \quad \text { and } \quad\left\{\gamma_{5}, \gamma_{\mu}\right\}=0 \quad \text { for } \quad \mu \in\{0,1,2,3\}
$$

It can be shown that a current with $\gamma_{5}$ transforms as a pseudoscalar under the parity operator. Thus a weak current that includes $\gamma_{5}$ allows for parity violation in the weak interaction. This is illustrated by Equation 2.14.

$$
\begin{aligned}
\hat{\bar{\psi}}_{1 \mathbf{P}}(\mathbf{x}, t) \gamma_{5} \hat{\psi}_{2 \mathbf{P}}(\mathbf{x}, t) & =\hat{\psi}_{1 \mathbf{P}}^{\dagger}(\mathbf{x}, t) \gamma^{0} \gamma_{5} \hat{\psi}_{2 \mathbf{P}}(\mathbf{x}, t) \\
& =\hat{\psi}_{1}^{\dagger}(-\mathbf{x}, t) \gamma^{0} \gamma^{0} \gamma_{5} \gamma^{0} \hat{\psi}_{2}(-\mathbf{x}, t) \\
& =-\hat{\psi}_{1}(-\mathbf{x}, t) \gamma_{5} \hat{\psi}_{2}(-\mathbf{x}, t) \quad \text { where } \quad \gamma^{0} \gamma_{5}=-\gamma_{5} \gamma^{0}
\end{aligned}
$$

Currents of the form $\hat{\bar{\psi}}_{1}(\mathbf{x}, t) \gamma^{\mu} \gamma_{5} \hat{\psi}_{2}(\mathbf{x}, t)$ transform as axial 4-vectors under the parity operator. Where the time component of axial 4-vectors transforms as a pseudoscalar and the spatial component transforms as a axial vector. All of the possible forms of the weak interaction are shown in Table 2.1.

\begin{tabular}{|c|c|c|}
\hline Interaction Type & Form of Current & Parity \\
\hline Scalar & $\hat{\bar{\psi}} \hat{\psi}$ & Even \\
\hline Pseudoscalar & $\hat{\bar{\psi}} \gamma_{5} \hat{\psi}$ & Odd \\
\hline Vector & $\hat{\hat{\psi}} \gamma^{\mu} \hat{\psi}$ & Odd \\
\hline Axial Vector & $\hat{\bar{\psi}} \gamma^{\mu} \gamma_{5} \hat{\psi}$ & Even \\
\hline Tensor & $\frac{1}{2} \hat{\bar{\psi}}\left(\gamma^{\mu} \gamma^{v}-\gamma^{v} \gamma^{\mu}\right) \hat{\psi}$ & Odd \\
\hline Pseudotensor & $\frac{1}{2} \hat{\bar{\psi}}\left(\gamma^{\mu} \gamma^{v}-\gamma^{v} \gamma^{\mu}\right) \gamma_{5} \hat{\psi}$ & Even \\
\hline
\end{tabular}

Table 2.1: The possible forms of the weak interaction that are allowed in Dirac's theory

A year later in 1957 it was confirmed that parity symmetry was violated by Wu et al [16] in the $\beta$-decay of ${ }^{60} \mathrm{Co}$. Soon after this discovery it was realized that Fermi's original current-current interaction was in fact a combination of a vector (V) type current and an axial-vector (A) type current. This led to the "VA" (vector minus axial-vector) structure of the weak interaction, which involved 
changing the original 4-vector current from Fermi to:

$$
\bar{u}_{e^{-}} \gamma \mu u_{v} \rightarrow \bar{u}_{e^{-}} \gamma_{\mu}\left(1-\gamma_{5}\right) u_{v}
$$

This V-A structure of the weak interaction is a fundamental part of the Standard Model. It has important implications for the way the left-handed (spin opposite the direction of motion) vs. right-handed (spin in the direction of motion) components of fermions (such as the neutrino) participate in the weak interaction.

\subsubsection{Helicity and Chirality}

The projection of the spin of a particle in the direction of the particles motion is called the particles helicity. Thus the helicity operator is:

$$
h=\frac{1}{2} \boldsymbol{\sigma} \cdot \hat{\mathbf{p}} \quad \text { where } \quad \hat{\mathbf{p}}=\frac{\mathbf{p}}{|\mathbf{p}|}
$$

Chirality is the sign of the helicity operator. Chirality is fundamental to the weak interaction. Left-handed particles have negative chirality and right-handed particles have positive chirality. In the Pauli-Dirac representation of the $\gamma$ matrices the $\gamma_{5}$ matrix is:

$$
\gamma_{5}=\left(\begin{array}{cc}
\mathbf{0} & \mathbf{I}_{\mathbf{2}} \\
\mathbf{I}_{\mathbf{2}} & \mathbf{0}
\end{array}\right)
$$

Applying the $\gamma_{5}$ operator to the spinor solutions from Equation 2.9 yields:

$$
\gamma_{5}\left(\begin{array}{l}
u_{a} \\
u_{b}
\end{array}\right)=\left(\begin{array}{cc}
\mathbf{0} & \mathbf{I}_{2} \\
\mathbf{I}_{2} & \mathbf{0}
\end{array}\right)\left(\begin{array}{c}
\phi \\
\frac{\boldsymbol{\sigma} \cdot \mathbf{p}}{E+m} \phi
\end{array}\right)=\left(\begin{array}{c}
\frac{\boldsymbol{\sigma} \cdot \mathbf{p}}{E+m} \phi \\
\boldsymbol{\phi}
\end{array}\right)
$$

Applying the relativistic approximation $(E \rightarrow|\mathbf{p}|$ as $m \rightarrow 0)$ gives:

$$
\gamma_{5}\left(\begin{array}{c}
u_{a} \\
u_{b}
\end{array}\right)=\left(\begin{array}{c}
(\boldsymbol{\sigma} \cdot \hat{\mathbf{p}}) \phi \\
\phi
\end{array}\right)=\left(\begin{array}{c}
(\boldsymbol{\sigma} \cdot \hat{\mathbf{p}}) \phi \\
(\boldsymbol{\sigma} \cdot \hat{\mathbf{p}})^{2} \phi
\end{array}\right)
$$


thus:

$$
\gamma_{5}\left(\begin{array}{l}
u_{a} \\
u_{b}
\end{array}\right)=\left(\begin{array}{cc}
\boldsymbol{\sigma} \cdot \hat{\mathbf{p}} & 0 \\
0 & \boldsymbol{\sigma} \cdot \hat{\mathbf{p}}
\end{array}\right)\left(\begin{array}{l}
u_{a} \\
u_{b}
\end{array}\right)
$$

Equation 2.20 reveals that the $\gamma_{5}$ operator approaches the helicity operator as the mass of a particle goes to zero. The neutrino is assumed to be massless within the Standard Model and thus the neutrino's helicity is the same as the neutrino's chirality. Left-handed chirality massive particles will have mostly left-handed helicity (with some right-handed helicity) and right-handed chirality massive particles will have mostly right-handed helicity (with some left-handed helicity). This give the helicity projection operators:

$$
P_{L} \equiv\left(\frac{1-\gamma_{5}}{2}\right) \quad, \quad P_{R} \equiv\left(\frac{1+\gamma_{5}}{2}\right)
$$

The helicity projection operators satisfy the following relations:

$$
P_{R}^{2}=P_{R} \quad, \quad P_{L}^{2}=P_{L} \quad, \quad P_{R} P_{L}=P_{L} P_{R}=0 \quad, \quad P_{R}+P_{L}=1
$$

The left, and right handed components of the Dirac spinors can then be defined as:

$$
u_{L} \equiv P_{L} u \quad, \quad u_{r} \equiv P_{R} u
$$

which allows for the rewriting of the V-A current between fermionic Dirac spinors as:

$$
\begin{aligned}
\bar{u}_{1} \gamma^{\mu} \frac{1-\gamma_{5}}{2} u_{2} & =\bar{u}_{1} \gamma^{\mu} P_{L} u_{2}=\bar{u}_{1} \gamma^{\mu} P_{L}^{2} u_{2} \\
& =\bar{u}_{1} \gamma^{\mu} P_{L} u_{2 L}=\bar{u}_{1} P_{R} \gamma^{\mu} u_{2 L} \\
& =\bar{u}_{1}^{\dagger} P_{L} \gamma^{0} \gamma^{\mu} u_{2 L}=\bar{u}_{1 L} \gamma^{\mu} u_{2 L}
\end{aligned}
$$

Equation 2.24 demonstrates the V-A structure of the theory of weak interactions. Equation 2.24 implies that only the left-handed component of the chirality of fermions participates in the weak interaction. This can also be shown for right 
handed chirality anti fermions. Additionally, because the helicity operator transforms as a pseudoscalar under parity, it can be shown that the V-A structure of weak interactions also implies that all massive fermions have the positive helicity component suppressed by a factor of order $m / E$, and similarly the negative helicity component of massive fermions is also suppressed by the same factor.

The Standard Model makes no prediction about the helicity of neutrinos, however the neutrino was assumed to be massless, and thus would have to have either fully positive helicity or fully negative helicity. In 1958 capture of electrons on ${ }^{152} \mathrm{Eu}$ showed that the helicity of the emitted neutrinos was $100 \%$ negative (within experimental uncertainties)[17]. This result gave strong evidence for the V-A description of weak interactions, and also gave strong confirmation for the massless neutrino of the Standard Model.

\subsubsection{Electroweak Gauge Theory}

Though the V-A theory was quite successful at describing the weak interaction, it still assumed a current-current interaction which caused a lot of theoretical issues. By the 1960s theoretical physicists were working on a gauge theory of the weak interaction. A weak gauge theory would consist of the introduction of a weak gauge symmetry group, and a corresponding intermediate vector boson field. The vector boson field is needed to keep the Lagranian invariant under certain local transformations. The weak gauge theory allowed for the unification of the weak force with the electromagnetic force, then through spontaneous symmetry breaking and the Higgs mechanism, the intermediate particles aquired masses becoming the Standard Model vector bosons: photon $(\gamma), \mathrm{Z}^{0}$ and $\mathrm{W}^{ \pm}$.

The gauge theory of the weak interaction views $e_{L}^{-}$and $v_{e L}$ as two states of the same 'particle' under the charged current (CC) processes. Assuming the particles $e_{L}^{-}$and $v_{e L}$ are different states of the same underlying particle suggests that this pair transform as a doublet under some symmetry group, a similar transformation property would also hold for the pairs, $\mu_{L}^{-} \leftrightarrow v_{\mu L}, \tau_{L}^{-} \leftrightarrow v_{\tau L}$. The $S U$ (2) group was originally proposed as the weak interaction group by Glashow [18], then expanded 
upon by Weinberg [19] and Salam [20].

The weak interaction group is usually labeled $S U(2)_{L}$ to denote the fact that it is only the left-handed component of the fields that participate in the weak interaction, and also to distinguish it from the standard $S U(2)$ group. Within the weak gauge theory the left-handed component of the fields that enter into the weak interaction corresponds to transformations in the internal space of the weak isospin. The $S U(2)$ group is an isomorphic group to the $S O(3)$ group, thus these transformations can be considered to be rotations in a three-dimensional weak isospin-space. $I$ and $I_{3}$ are used to denote the quantum numbers of weak isospin, which have the following assignments for the leptonic fields:

$$
I=\frac{1}{2} \quad\left\{\begin{array}{l}
I_{3}=+1 / 2 \\
I_{3}=-1 / 2
\end{array} \quad\left(\begin{array}{c}
\hat{\mathrm{v}}_{e} \\
\hat{e}^{-}
\end{array}\right)_{L}\left(\begin{array}{c}
\hat{\mathrm{v}}_{\mu} \\
\hat{\mu}^{-}
\end{array}\right)_{L}\left(\begin{array}{c}
\hat{\mathrm{v}}_{\tau} \\
\hat{\tau}^{-}
\end{array}\right)_{L}\right.
$$

These transformations can be rewritten as:

$$
\left(\begin{array}{c}
\hat{\mathrm{v}}_{e} \\
\hat{e}^{-}
\end{array}\right)_{L}^{\prime}=e^{-i \frac{\alpha \cdot \tau}{2}}\left(\begin{array}{c}
\hat{\mathrm{v}}_{e} \\
\hat{e}^{-}
\end{array}\right)_{L}
$$

where $\tau$ denotes Pauli spin matrices that act in the internal isospin space. This group should be considered as locally gauge invariant, thus local transformations $\boldsymbol{\alpha}\left(x^{\mu}\right)$ are allowed, without changing the observed physics. This group introduces three gauge fields (one for each of the three axes in weak isospin-space); two of the fields should have 'charge' \pm 1 and the third should be a neutral field. The charged fields allow for transitions between the doublet members. The charged fields corresponds to the charged current type interactions while the neutral field corresponds to the neutral current (NC) interactions. NC interactions were first observed in 1973 by the Gargamelle bubble chamber experiment at CERN [21]. It was learned from the Gargamelle experiment that NC interactions are not pure VA, thus the neutral gauge field of the weak interaction, is not completely described by the V-A theory of the weak interaction. 
The proposed solution was the unification of the weak force with the electromagnetic force, this was done via the addition of an extra $U(1)$ gauge group which resulted in a new $S U(2)_{L} \otimes U(1)$ structure for the unified electroweak force. The new gauge group needed to include a mechanism to deal with the right-handed electron (for electromagnetic interactions). Because the V-A structure works just fine for $\mathrm{CC}$ weak interactions, this new mechanism needs to be a singlet in weak isospin-space. As an example consider the first generation of leptons and quarks, arranged into two $S U(2)$ doublets:

$$
\left(\begin{array}{c}
\hat{\mathrm{v}}_{e} \\
\hat{e}^{-}
\end{array}\right)_{L} \quad\left(\begin{array}{c}
\hat{u} \\
\hat{d}
\end{array}\right)_{L}
$$

and three $S U(2)$ singlets; $\hat{e}_{R}^{-}, \hat{u}_{R}, \hat{d}_{R}$. The weak hypercharge, $Y$, was introduced to differentiate between left-handed doublet and right-handed singlet particles. The weak hypercharge is defined as:

$$
Y=2 Q-2 I_{3}
$$

where $Q$ is the electric charge and $I_{3}$ the third component of weak isospin. Both the weak isospin and the local phase change fromt he $U(1)$ transform should be local gauge symmetries, thus local transformations should not change the observed physics. This can be expressed as:

$$
\hat{\chi}_{L}^{\prime}=\exp \left(i g_{w} \frac{\boldsymbol{\alpha}\left(x^{\mu}\right) \cdot \tau}{2}+i g^{\prime} \gamma^{0}\left(x^{\mu}\right) Y\right) \hat{\chi}_{L}
$$

where $\hat{\chi}_{L}$ is a left-handed chiral doublet, $g_{w}$ and $g^{\prime}$ are coupling constants and $x^{\mu}$ is a space-time point. The Lagrangian must be kept invariant under the local $S U(2) \otimes U(1)$ transformation, this is done by introducing four new gauge fields; two charged fields $\left(W_{1,2}^{\mu}\right)$ and one neutral field $\left(W_{3}^{\mu}\right)$ for the $S U(2)$ part of the symmetry group, a second neutral field $\left(B^{\mu}\right)$ for the $U(1)$ part of the symmetry group. Just as in the V-A theory of weak interactions the charged fields are re- 
sponsible for raising and lowering of the left-handed chiral doublets, and the two neutral fields will account for the weak NC interaction and the electromagnetic interaction respectively. This gauge invariant Lagrangian can be written as:

$$
\begin{aligned}
\hat{\bar{\chi}}_{L} \gamma^{\mu}\left(i \partial_{\mu}-g_{w} \frac{\tau}{2} \cdot \hat{\mathbf{W}}_{\mu}-\frac{g^{\prime}}{2} Y \hat{B}_{\mu}\right) \hat{\chi}_{L} & +\hat{\bar{\chi}}_{R} \gamma^{\mu}\left(i \partial_{\mu}-\frac{g^{\prime}}{2} Y \hat{B}_{\mu}\right) \hat{\chi}_{R} \\
& -\frac{1}{4} \hat{W}_{\mu v} \hat{W}^{\mu v}-\frac{1}{4} \hat{B}_{\mu v} \hat{B}^{\mu v}
\end{aligned}
$$

In equation 2.30 the final two terms are the self-interactions of the introduced gauge fields and the right-handed chiral fields interact only via the $\hat{B}^{\mu}$, this makes the electromagnetic force free form the $\gamma_{5}$ and parity violating terms.

There are no mass terms in Equation 2.30 (the electroweak Lagrangian) this is because by gauge fields must be mass less to obey gauge invariance. However, through the process of spontaneous symmetry breaking the massless $\hat{W}_{1}^{\mu}$ and $\hat{W}_{2}^{\mu}$ combine to form the massive $\hat{W}^{ \pm}$fields and the massless $\hat{W}_{3}^{\mu}$ and $\hat{B}^{\mu}$ combine to form the massive $\hat{Z}^{0}$ and the massless photon fields. These fields are viewed as propagators, exchanged virtual bosons, and are used in Standard Model Feynman diagrams, shown in Figure 2.1. The matrix element for the transition shown in

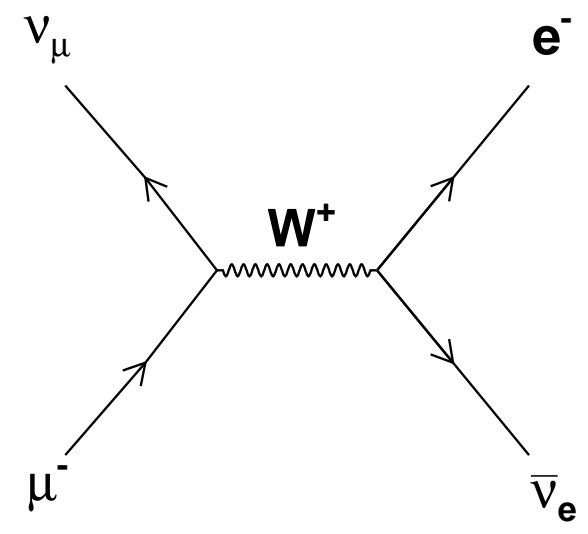

Figure 2.1: Feynman diagram of the muon decay process $\left(\mu^{-} \rightarrow v_{\mu}+\right.$ $\left.\overline{\mathrm{v}}_{e} e^{-}\right)$ 
Figure 2.1 written in terms of Dirac spinors and the exchanged virtual bosons is:

$$
\left.\left[-\frac{i g_{w}}{\sqrt{2}} \bar{u}_{v_{\mu}} \frac{1}{2} \gamma^{\mu}\left(1-\gamma_{5}\right) u_{\mu^{-}}\right]\left[i \frac{-g^{\mu \nu}+q^{\mu} q^{v} / M_{W}^{2}}{q^{2}-M_{W}^{2}}\right]\left[\frac{i g_{w}}{\sqrt{2}} \bar{u}_{e^{-}} \frac{1}{2} \gamma_{\mu}\left(1-\gamma_{5}\right) u_{e^{-}}\right)\right]
$$

where $g_{w} / \sqrt{2}$ is the coupling strength at the vertexes, $g^{\mu v}$ is the metric tensor and $q^{2}$ the squared four-momentum transfer between the vertices.

At low $q^{2}\left(q^{2} \ll M_{W}^{2}\right)$ the W-propagator can be replaced by the constant term $g^{\mu v} / M_{W}^{2}$ leading to the matrix element shown in Equation 2.33. Equation 2.33 is very similar to the current-current form of the weak interaction. Thus the V-A current-current form is the low energy approximation of the full GlashowWeinburg-Salam gauge theory of electroweak interactions.

$$
\begin{gathered}
-\frac{i g_{w}^{2}}{8 M_{W}^{2}} \bar{u}_{v_{\mu}} \frac{1}{2} \gamma^{\mu}\left(1-\gamma_{5}\right) u_{\mu^{-}}-\bar{u}_{e^{-}} \frac{1}{2} \gamma^{\mu}\left(1-\gamma_{5}\right) u_{e^{-}} \\
\text {where } \quad \frac{G_{F}}{\sqrt{2}}=\frac{g_{w}^{2}}{8 M_{W}^{2}}
\end{gathered}
$$

Simple leptonic scattering in the Standard Model can be built from V-A current involving Dirac spinors, this is demonstrated by Equation 2.33 which shows how to calculate the V-A transition amplitude. All of the known leptonic pairs undergo this form of the V-A coupling, and have the same 'strength parameter'. This property of weak interactions means that cross sections for all of the completely leptonic scattering interactions can be computed using the formalism from Equation 2.33. The simpleness of this formalism is complicated when the target of the neutrino scattering lies within a nucleon or the nucleon is bound within a nucleus. 


\subsection{Neutrino-Nucleus Scattering}

\subsubsection{Neutrino Scattering Off of Nucleons}

When the momentum transferred by the weak boson is low $\left(q^{2} \ll M_{W}^{2}\right)$, the pure $\mathrm{V}$-A description of weak interactions is a valid approximation. Even though the previous discussion of weak interactions only addressed the lepton sector, the V-A structure is also a valid description of vertices with a weak current between quarks such as:

$$
\bar{u}_{u} \gamma_{\mu}\left(1-\gamma_{5}\right) u_{d}
$$

Complications arise when going from the vertices involving lone quarks to vertices involving multiple quarks bound together in a nucleon, however. When considering neutrino scattering off of nucleons, it is important to consider additional strong interaction effects. Unlike the leptons (and quarks), the nucleons (proton and neutron) cannot be described as point like particles, consequently the internal structure of the nucleons must be included in any description of scattering off of nucleons. Describing the effects of the nucleon internal structure involves the use of quantum chromodynamics (QCD). QCD allows for many additional processes such as quark-antiquark pair production from gluons and gluon emission from valence quarks. Even with these additional complications from QCD the total electric charge must be always be conserved (the proton always has a charge of $e$ ), however there is no reason to assume this is also true for the weak interaction. As an example, consider the formation of quark-antiquark pair, the net contribution to the weak interaction from this quark-antiquark pair may not be zero.

Ignoring for a moment intermediate vector bosons and $q^{2}$ considerations, the strong interaction effects can be accounted for by making the following replacement in the weak current:

$$
\left(1-\gamma_{5}\right) \rightarrow\left(c_{V}-c_{A} \gamma_{5}\right)
$$


Here $c_{V}$ and $c_{A}$ are experimentally determined correction factors. These correction factors have been measured through $\beta$-decay experiments to be:

$$
c_{V}=1.000 \pm 0.003 \quad, \quad c_{A}=1.26 \pm 0.02
$$

Equation 2.36 demonstrates that the vector part of the weak current is not modified by the QCD structure of the nucleon. This indicates that there may be some conservation law that 'protects' the vector current in the same way that the electromagnetic charge is protected. This conservation law is known as the Conserved Vector Current (CVC) hypothesis, which was first proposed by Feynman and GellMann [22]. They speculated that the vector part of the weak current $\bar{u}_{p} \gamma_{\mu} u_{n}$, its conjugate current $\bar{u}_{n} \gamma_{\mu} u_{p}$, and the electromagnetic current $\bar{u}_{p} \gamma_{\mu} u_{p}$ form a triplet of conserved currents in the internal isospin space of the strong interaction.

\subsubsection{Neutrino-Nucleon Scattering Kinematics}

It will be easier to discuss the theory of neutrino-nucleon scattering by first addressing the general kinematic quantities of $\mathrm{CC}$ neutrino-nucleon scattering. Figure 2.2 shows a diagram for the scattering process $v_{\mu}+N \rightarrow \mu^{-}+X$ (here $X$ represents the hadronic final state). Figure 2.2 labels the measured quantities for an event detected using in the MINOS detectors and the center of mass frame four-momentum of the particles in the interaction.

Table 2.2 lists how to calculate the Lorentz invariant kinematic quantities that describe a general $\mathrm{CC} v_{\mu}-N$ interaction. The calculations are shown for both the center of momentum frame (using four-momentum quantities), and for the lab frame (detector measured quantities).

In quasi-elastic scattering (QE) event, $v_{\mu}+n \rightarrow \mu^{-}+p$, the neutrino is considered to scatter off of the entire nucleon, and not the individual partons. In a QE interaction the target nucleus does not break up but is just modified. If an interaction is considered to be quasi-elastic then the hadronic system will consist of a single proton $\left(W^{2}=M_{\text {proton }}^{2}\right)$. QE scattering can be contrasted with resonances 


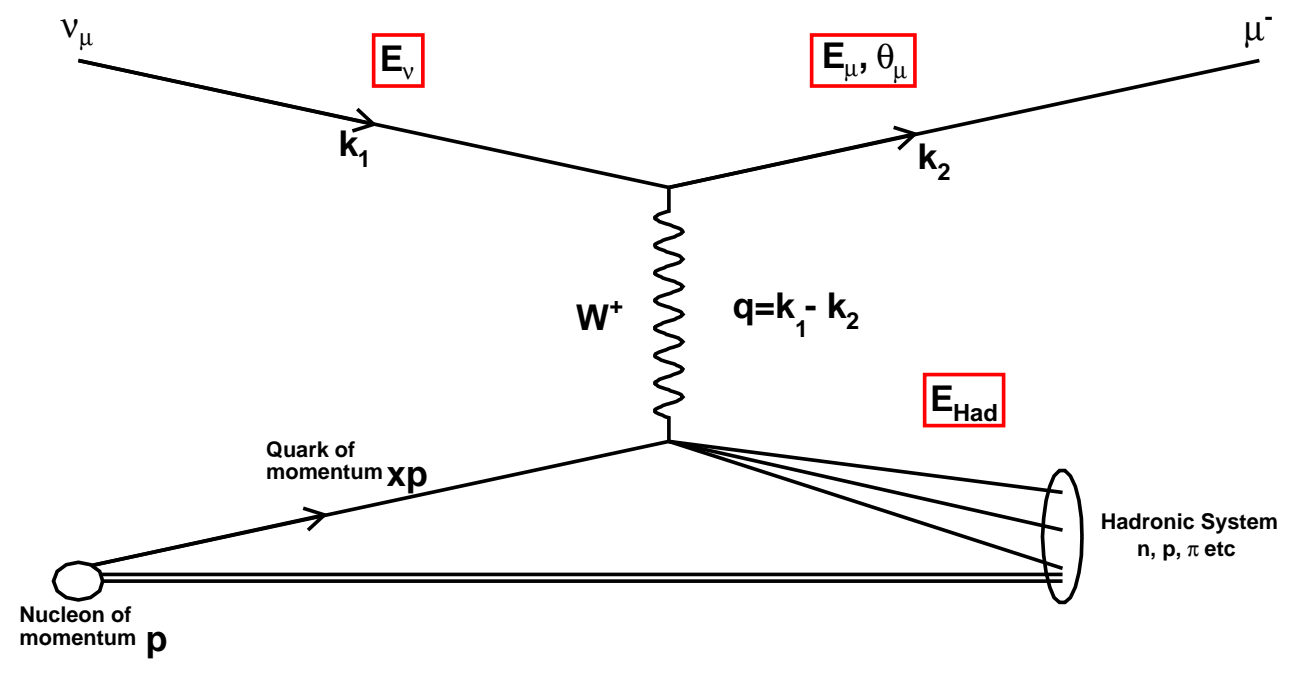

Figure 2.2: $\mathrm{CC}_{\mu}-N$ scattering kinematics. Inside the red boxes are the variables that are measured in the MINOS detectors (in the lab frame). The diagram also labels the four-momentum of the particles in the center of mass frame. The Bjorken scaling variable, $x$, denotes the fraction of the nucleon momentum carried by the struck quark.

production (Res) and deep inelastic scattering (DIS) which involve ever deeper probing of the quark structure of the nucleon.

Resonances production, $v_{\mu}+N \rightarrow \mu^{-}+\Delta \rightarrow \mu^{-}+\pi+N^{\prime}$, occurs when the scattering of the neutrino off of the nucleon, excites the nucleon into a higher energy state called resonance particle (usually a $\Delta(1232)$. The $\Delta$ quickly decays into a pion and a nucleon.

Deep inelastic scattering, $v_{\mu}+N \rightarrow \mu^{-}+X$ (hadrons), occurs when the neutrino penetrates the nucleon and interacts with just one of the constituent quarks. This knocks the quark out of the nucleon, however due to color confinement bare quarks are not allowed, this causes the creation of a hadronic shower.

One of the consequences of assuming an interaction is a QE interaction is that several of the kinematic variables listed in Table 2.2 can be calculated using just 


\begin{tabular}{|c|c|c|}
\hline Variable Description & $\begin{array}{c}\text { Center of Momentum } \\
\text { Frame Calculation }\end{array}$ & $\begin{array}{c}\text { Lab Frame } \\
\text { Calculation }\end{array}$ \\
\hline \hline $\begin{array}{c}\text { Energy transfered to } \\
\text { the hadronic system: } v\end{array}$ & $\boldsymbol{p} \cdot \boldsymbol{q} / M$ & $E_{\text {had }}$ \\
\hline Inelasticity: $y$ & $(\boldsymbol{p} \cdot \boldsymbol{q}) /\left(\boldsymbol{p} \cdot \boldsymbol{k}_{1}\right)$ & $E_{\text {had }} / E_{\mathrm{v}}$ \\
\hline $\begin{array}{c}\text { Squared four-momentum } \\
\text { transfer: } Q^{2}\end{array}$ & $-\boldsymbol{q}^{2}$ & $2 E_{\mathrm{v}} E_{\mu}\left(1-\cos \left(\theta_{\mu}\right)\right)$ \\
\hline Bjorken scaling variable: $x$ & $Q^{2} / 2 \boldsymbol{p} \cdot \boldsymbol{q}$ & $Q^{2} / 2 E_{\text {had }} M$ \\
\hline $\begin{array}{c}\text { Squared invariant mass of } \\
\text { of the hadronic system: } W^{2}\end{array}$ & $(\boldsymbol{p}+\boldsymbol{q})^{2}$ & $M^{2}+2 E_{\text {had }} M-Q^{2}$ \\
\hline
\end{tabular}

Table 2.2: Lorentz invariant kinematic variables that describe the charged current neutrino-nucleon scattering. The mass $M$ is the mass of the struck nucleon.

the measured quantities that correspond to the outgoing muon. The QE-assumed kinematic quantities introduced here along with the quantities in Table 2.2 will be used extensively in the rest of this thesis.

- Neutrino Energy: In MINOS the usual way to reconstruct the neutrino energy is by summing the visible energy in the detector for a given event, $E_{v}=E_{\mu}+E_{\text {had }}$, where $E_{\text {had }}$ is calculated from the sum of the visible energy not originated from the muon. However if an event is assumed to be due to a QE interaction then a QE-assumed neutrino energy can be calculated instead. This neutrino energy can be calculated using just the measured muon kinematics according to:

$$
E_{v}^{Q E}=\frac{E_{\mu} M-\left(M_{\mu}^{2}\right)}{M-E_{\mu}+p_{\mu} \cos \left(\theta_{\mu}\right)}
$$

here $M$ is the mass of the struck nucleon, $M_{\mu}$ is the mass of the out going muon. Equation 2.37 neglects terms involving the binding energy of the struck nucleon. 
- Squared four-momentum transfer: The QE-assumed four-momentum transfer from the lepton to the hadronic system, it can also be calculated using just the outgoing muon kinematics from:

$$
Q_{Q E}^{2}=-2 E_{v}^{Q E}\left(E_{\mu}-p_{\mu} \cos \left(\theta_{\mu}\right)\right)+M_{\mu}^{2}
$$

\subsubsection{Nucleon Form Factors and the QE Cross Section}

A $v_{\mu}$-CC QE scattering interaction can be described by:

$$
\mathrm{v}_{\mu}(p)+n(P) \rightarrow \mu^{-}\left(p^{\prime}\right)+p\left(P^{\prime}\right)
$$

where $p\left(p^{\prime}\right)$ is the incoming (outgoing) momentum of the lepton and $P\left(P^{\prime}\right)$ is the incoming (outgoing) momentum of the nucleon. The Feynman diagram for this interaction is shown in Figure 2.3. The matrix element for this interaction

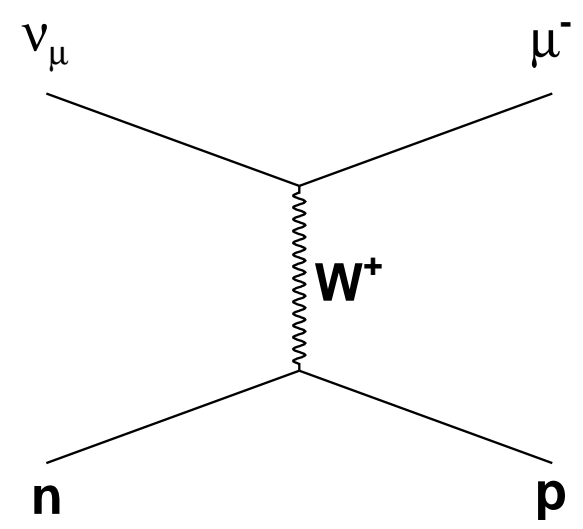

Figure 2.3: Feynman diagram of the $v_{\mu}$-CC QE interaction, showing both the $v_{\mu}, W^{+}, \mu^{-}$and the $n, W^{+}, p$ vertices.

can be calculated by modifying Equation 2.33 using equation 2.34 then including the further effects of having the quarks bound within a nucleon. This is given in 
Equation 2.40:

$$
\frac{G_{F}}{\sqrt{2}} u_{\mu^{-}}^{-}\left(p^{\prime}\right) \gamma_{\mu}\left(1-\gamma_{5}\right) u_{v_{\mu}}(p) \cos \left(\theta_{C}\right) \bar{u}_{p}\left(P^{\prime}\right) \Gamma_{C C}^{\mu}\left(q^{2}\right) u_{n}(P)
$$

where $\theta_{C}$ is the Cabibbo angle (which measures the probability of quark flavor change due to the weak interaction [23] [24]) and $\Gamma_{C C}^{\mu}\left(q^{2}\right)$ is a term that contains the complex weak nucleon form factors given by Equation 2.41:

$$
\begin{aligned}
\Gamma_{C C}^{\mu} & =\gamma^{\mu} F_{V}^{2}\left(q^{2}\right)+\frac{i \sigma^{\mu v} q^{v}}{2 M} \xi F_{V}^{2}\left(q^{2}\right)+\frac{q_{\mu}}{M} F_{S}\left(q^{2}\right) \\
& +\left(\gamma^{\mu} F_{A}\left(q^{2}\right)+\frac{i \sigma^{\mu v} q^{v}}{2 M} F_{T}\left(q^{2}\right)+\frac{q_{\mu}}{M} F_{P}\left(q^{2}\right)\right) \gamma_{5}
\end{aligned}
$$

where $M$ is the mass of the struck nucleon. Each term in $\Gamma_{C C}^{\mu}$ is a form factor that parameterizes the amount of each type of weak current participating the interaction. The form factors are functions of the vector boson 'probe' squared four-momentum, $q^{2}$, which reflects the fact that the vector boson interacts with the nucleon internal structure at different 'levels' depending on the bosons $q^{2}$. These form factors are related to the correction factors of Equation 2.35, $c_{V}$ and $c_{A}$, in the $q^{2}=0$ limit.

Where $\xi=k_{p}-k_{n}+1$, with $k_{p} / k_{n}$ being the anomalous magnetic moments of the proton and neutron. Examining Table 2.1 the V-type nucleon from factors are: the vector form factors $F_{V}^{1}, F_{V}^{2}$, and the scalar form factor $F_{S}$ (which doesn't contribute to the weak interaction). In the standard model the A-type nucleon form factors are: the axial-vector form factor $F_{A}$, the pseudotensor form factor $F_{T}$ (which doesn't contribute to the weak interaction), and the pseudoscalar form factor $F_{P}$.

Llewellyn-Smith explained why the nucleon form factors must be real. In [25], Llewellyn-Smith pointed out that time reversal symmetry $(\mathrm{T})$ requires that, all of the nucleon form factors must be real and charge-exchange symmetry $(\mathrm{C})$ requires 
$F_{S}$, and $F_{T}$ to be imaginary. Thus $F_{S}$, and $F_{T}$ must be zero:

$$
F_{S}\left(q^{2}\right)=F_{T}\left(q^{2}\right)=0 \quad \forall q^{2}
$$

The muon mass multiplies the nucleon form factor $F_{P}$, thus for neutrino energies where $m_{\mu}^{2} \ll E_{v}^{2}$ the pseudoscalar from factor can be neglected. Setting $F_{S}$ and $F_{T}$ equal to 0 and neglecting the pseudoscalar form factor turns Equation 2.41 into:

$$
\Gamma_{C C}^{\mu}=\gamma^{\mu}\left(F_{V}\left(q^{2}\right)-\gamma_{5} F_{A}\left(q^{2}\right)\right)+\frac{i \sigma^{\mu v} q^{\mu}}{2 M} \xi F_{V}^{2}\left(q^{2}\right)
$$

Inserting Equation 2.43 into Equation 2.40 yields the full expression for the matrix element:

$$
\begin{aligned}
\frac{G_{F}}{\sqrt{2}} u_{\mu^{-}}^{-}\left(p^{\prime}\right) \gamma_{\mu}\left(1-\gamma_{5}\right) u_{v_{\mu}}(p) \cos \left(\theta_{C}\right) \bar{u}_{p}\left(P^{\prime}\right) & \left(\gamma^{\mu}\left(F_{V}^{1}\left(q^{2}\right)-\gamma_{5} F_{A}\left(q^{2}\right)\right)\right. \\
& \left.+\frac{i \sigma^{\mu v} q^{v}}{2 M} \xi F_{V}^{2}\left(q^{2}\right)\right) u_{n}(P)
\end{aligned}
$$

Turning the reduced form of the matrix element (Equation 2.44) into a differential cross section with respect to $q^{2}$, for QE scattering yields Equations 2.45 and 2.46.

$$
\begin{gathered}
\frac{d \sigma}{d\left|q^{2}\right|}=\frac{M^{2} G_{F}^{2} \cos ^{2}\left(\theta_{c}\right)}{8 \pi E_{v}^{2}}\left[A\left(q^{2}\right)-B\left(q^{2}\right) \frac{s-u}{M^{2}}+C\left(q^{2}\right) \frac{(s-u)^{2}}{M^{4}}\right] \\
\frac{d \sigma}{d\left|q^{2}\right|}=\frac{M^{2} G_{F}^{2} \cos ^{2}\left(\theta_{c}\right)}{8 \pi E_{v}^{2}}\left[A\left(q^{2}\right)+B\left(q^{2}\right) \frac{s-u}{M^{2}}+C\left(q^{2}\right) \frac{(s-u)^{2}}{M^{4}}\right]
\end{gathered}
$$

where Equation 2.45 is for the interaction $v+n \rightarrow l^{-}+p$, and Equation 2.46 is for the interaction $\bar{v}+p \rightarrow l^{-}+n$. In Equations 2.45 and $2.46 s-u=E_{\mathrm{v}} M-q^{2}-$ $m_{l}^{2}, m_{l}$ is the mass of the outgoing lepton and the $A\left(q^{2}\right), B\left(q^{2}\right), C\left(q^{2}\right)$ can be 
expressed as:

$$
\begin{gathered}
A\left(q^{2}\right)=\frac{m_{l}^{2}-q^{2}}{4 M^{2}}\left[\left(4-\frac{q^{2}}{M^{2}}\right)\left|F_{A}\right|^{2}-\left(4+\frac{q^{2}}{M^{2}}\right)\left|F_{V}^{1}\right|^{2}\right. \\
-\frac{q^{2}}{M^{2}}\left|\xi F_{V}^{2}\right|^{2}\left(1+\frac{q^{2}}{4 M^{2}}\right)-\frac{4 q^{2} \Re\left(F_{V}^{1} \xi F_{V}^{2}\right)}{M^{2}} \\
\left.-\frac{m_{l}^{2}}{M^{2}}\left(\left(F_{V}^{1}+\xi F_{V}^{2}\right)^{2}+\left|F_{A}\right|^{2}\right)\right] \\
B\left(q^{2}\right)=-\frac{q^{2}}{M^{2}}\left[\left(F_{V}^{1}+\xi F_{V}^{2}\right) \Re\left(F_{A}\right)\right] \\
C\left(q^{2}\right)=\frac{1}{4}\left(\left|F_{A}\right|^{2}+\left|F_{V}^{1}\right|^{2}-\frac{q^{2}}{4 M^{2}}\left|\xi F_{V}^{2}\right|^{2}\right)
\end{gathered}
$$

It is possible to further constrain the vector form factors of Equations 2.45 and 2.46 by considering $e+N$ elastic scattering. The nucleons cannot be described as point like particles, but in fact have an internal structure to both their charge distribution and their magnetic moment. In 1950 Rosenbluth [26] proposed treating the charge distribution and magnetic moment separately and describing the contribution to the cross section with form factors. It can be shown that when Rosenbluth's ideas are applied to electron-proton elastic scattering (for example in [27]) the electromagnetic current contribution to the hadronic vertex has this form:

$$
\bar{u}_{p, n}\left(P^{\prime}\right)\left[\gamma^{\mu} F_{p, n}^{1}\left(q^{2}\right)-\frac{\sigma^{\mu v} q^{v}}{2 M} F_{p, n}^{2}\left(q^{2}\right)\right] u_{n, p}(P)
$$

with $F_{p, n}^{1}$ describing the deviation of the nucleon from a point charge (the Dirac form factor), and $F_{p, n}^{2}$ describing the deviation of the nucleon from a 'pointlike' magnetic moment (the Pauli form factor).

The Sachs [28] form factors are defined in terms of the Dirac and Pauli form 
factors:

$$
\begin{aligned}
G_{E}^{p, n}\left(Q^{2}\right) & =F_{p, n}^{1}-\frac{Q^{2}}{4 M^{2}} F_{p, n}^{2}\left(Q^{2}\right) \\
G_{M}^{p, n}\left(Q^{2}\right) & =F_{p, n}^{1}+F_{p, n}^{2}\left(Q^{2}\right)
\end{aligned}
$$

Electron-nucleon scattering experiments have constrained these form factors with a great degree of precision while also establishing the dipole form for all of the form factors.

$$
G_{D}\left(Q^{2}\right)=\frac{1}{\left(1+\frac{Q^{2}}{M_{V}^{2}}\right)^{2}}
$$

where $M_{V}$ is the vector mass. Expressing the Sachs form factors in terms of the dipole form factor yields:

$$
\begin{aligned}
G_{E}^{p}\left(Q^{2}\right) & =G_{D}\left(Q^{2}\right) \\
G_{E}^{n}\left(Q^{2}\right) & =0 \\
G_{M}^{p}\left(Q^{2}\right) & =\mu_{p} G_{D}\left(Q^{2}\right) \\
G_{M}^{n}\left(Q^{2}\right) & =\mu_{n} G_{D}\left(Q^{2}\right)
\end{aligned}
$$

where $\mu_{n, p}$ is the magnetic moment of the proton or neutron. The vector mass $M_{V}$ in equation 2.52 is well constrained by electron scattering experiments.

By assuming the validity of the CVC hypothesis, the Sachs form factors can be used to define the weak nucleon vector form factors from Equation 2.43:

$$
\begin{gathered}
F_{V}^{1}\left(Q^{2}\right)=\frac{\left[G_{E}^{p}\left(Q^{2}\right)-G_{E}^{n}\left(Q^{2}\right)\right]+\frac{Q^{2}}{4 M^{2}}\left[G_{M}^{p}\left(Q^{2}\right)-G_{M}^{n}\left(Q^{2}\right)\right]}{1+\frac{Q^{2}}{4 M^{2}}} \\
F_{V}^{2}\left(Q^{2}\right)=\frac{\left[G_{M}^{p}\left(Q^{2}\right)-G_{M}^{n}\left(Q^{2}\right)\right]-\left[G_{E}^{p}\left(Q^{2}\right)-G_{E}^{n}\left(Q^{2}\right)\right]}{1+\frac{Q^{2}}{4 M^{2}}}
\end{gathered}
$$

Equations 2.54 and 2.55 demonstrate that the weak vector current form factors for the CC- $v_{\mu}$ QE scattering calculation can be constrained by the Sachs form factors 
using the CVC hypothesis. Because the Sachs form factors are well constrained by electron scattering experiments the only remaining unconstrained quantity in the CC- $v_{\mu}$ QE cross section calculation is the axial-vector form factor, $F_{A}\left(Q^{2}\right)$. The axial-vector form factor can be constructed in a similar manner, by analogy with the vector form factor the axial vector form factor is:

$$
F_{A}\left(Q^{2}\right)=\frac{F_{A}(0)}{\left(1+\frac{Q^{2}}{\left(M_{A}^{Q E}\right)^{2}}\right)^{2}}
$$

where the axial-vector mass is $M_{A}^{Q E}$, and $F_{A}\left(Q^{2}=0\right)$ has been measured in neutron $\beta$-decay experiments. Thus the only remaining quantity to be measured is the $Q^{2}$ behavior of the axial-vector form factor. Which, if the dipole form of the axial-vector form factor holds, is the same as measuring the axial-vector mass $M_{A}^{Q E}$.

Figure 2.4 shows the differential cross section with respect to $Q^{2}$ for the CC$v_{\mu} \mathrm{QE}$ interaction. The $Q^{2}$ distributions are for $1 \mathrm{GeV}$ energy neutrinos scattering off of a free nucleon.

\subsubsection{Nuclear Effects}

The previous discussion has only dealt with neutrino-nucleon scattering. However in MINOS the target nucleon lies within a nucleus (primarily iron), and this causes additional complications due to interactions with the other non-target nucleons within the nucleus. These effects can change both the cross-section, and the apparent final state particle multiplicity.

When the squared four-momentum of the vector boson probe $\left(W^{+}\right.$for CC- $v_{\mu}$ QE interactions) exceeds $\sim 0.2 \mathrm{GeV}^{2}$ it's wavelength is comparable to the diameter of an iron nucleus. Thus for $Q^{2}$ values less than $\sim 0.2 \mathrm{GeV}^{2}$ the boson probe does not just the target nucleon but is also affected by the internal structure of the nucleus. 

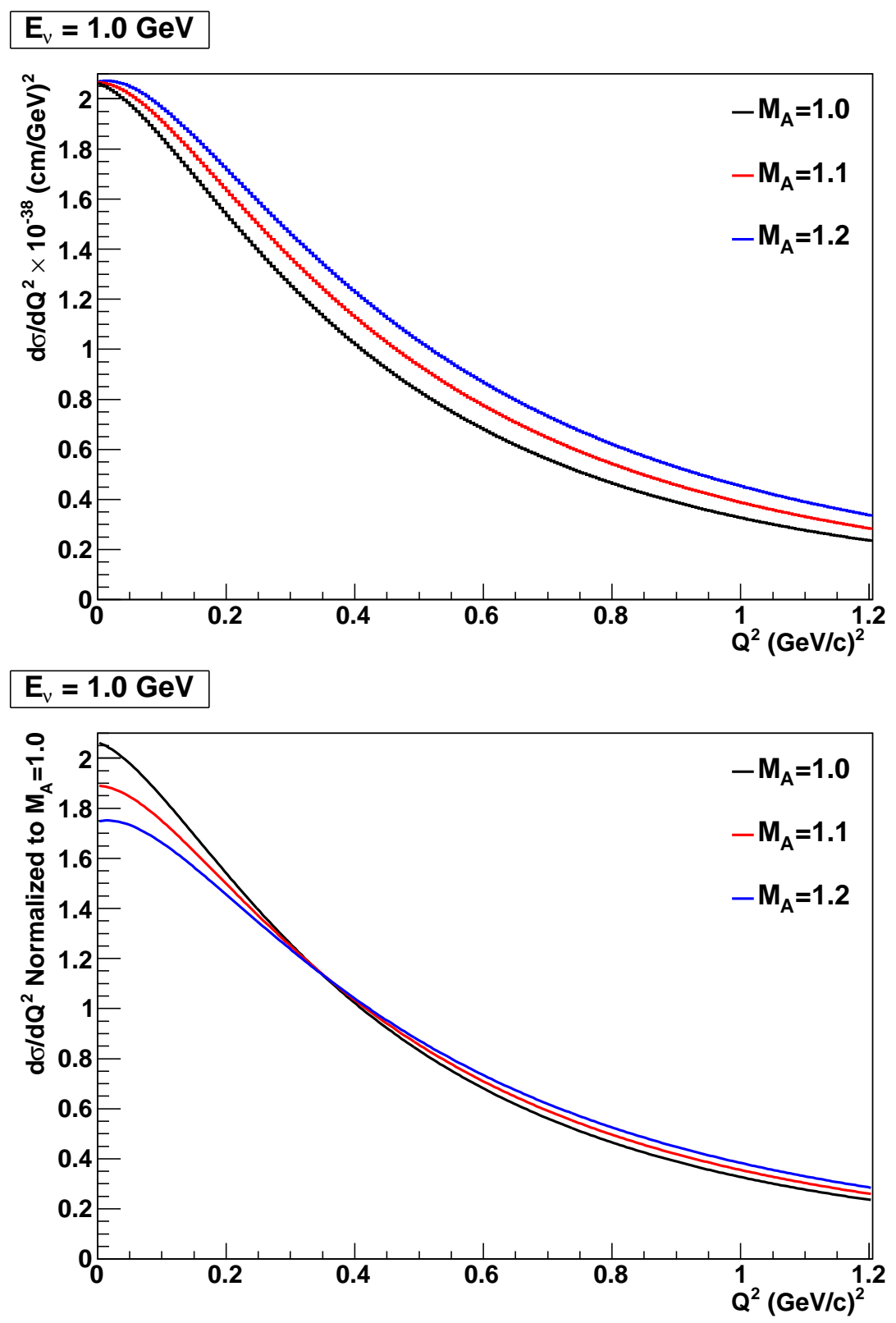

Figure 2.4: Above: quasi-elastic differential cross section off of a free nucleon with respect to $Q^{2}$. Each distribution corresponds to a different value of the axial-vector mass parameter. Below: The same $Q^{2}$ distribution as above except the red and blue distributions are normalized to the area of the black $Q^{2}$ distribution. 
Nuclear effects can change both the QE cross section $\left(\sigma_{Q E}\right)$, the kinematics of the final state, and the final state particle multiplicity. They include: the Fermi motion of the struck nucleon, the binding energy of the nucleus, Pauli-blocking (a consequence of the application of the Pauli exclusion principle to the nucleus), final state interactions (FSIs) (such as intranuclear re-scattering), and the Short Range Correlations (SRC) and Meson Exchange Currents (MEC) between nucleons.

\section{Pauli-Blocking}

Pauli-blocking is a consequence of the application of the Pauli exclusion principle to the interactions between nucleons within the nucleus. Under the Pauli exclusion principle it is not possible for any to fermions to occupy the same quantum state. This means that in a QE interaction it is not possible for the struck nucleon to be excited into a quantum state that is already occupied. Thus in a QE interaction the struck nucleus can only be excited if there is an unoccupied final state for the outgoing nucleon. Recent experiments have used the Fermi model to describe the the Pauli-blocking, and nucleon Fermi motion for QE scattering. In the Fermi Gas (FG) model the excitation of the nucleon is described as a transition across the Fermi surface, from below to above. The Fermi surface is a certain value of nucleon momentum (typically between 200-300 MeV) below which all of the quantum states are filled. The FG model assumes that the nucleus is composed of an infinite number of nucleons and that the nucleus is translationally invariant, with the momentum distribution of the nucleons given by:

$$
n(|\mathbf{p}|)=\frac{\tau}{\frac{4}{3} \pi k_{F}^{3}} \Theta\left(k_{F}-|\mathbf{p}|\right)
$$

where $\Theta$ is the Heavyside step function, $\tau$ is either the atomic number $Z$ or the neutron number $N$ for the nucleus in question, $k_{F}$ is the Fermi momentum and $\mathbf{p}$ is the three-momentum of the nucleon. When simulating the FG model all of the energy levels up to the Fermi surface are assumed to be filled. Thus any interaction 
where the transfered momentum excites the final state nucleon into a momentum state with less than the Fermi momentum is considered Pauli-blocked.

The FG model as it applies to QE neutrino-nucleus scattering was examined by Smith and Moniz in 1972 [29]. The FG model can have a large effect on both the QE cross section and the expected event rate at low values of $Q^{2}(<$ $0.2 \mathrm{GeV}^{2}$ ). Figure 2.5 shows several $Q^{2}$ distributions both without Pauli blocking (free nucleon) and with several values of the Fermi momentum.

The FG model of the nucleus improves agreement with neutrino-nucleus scattering data, relative to the free nucleon simulation, however the agreement is not perfect and more low $Q^{2}$ suppression by increasing the effective $k_{F}$ value may be required [30][31]. Progress has also be made by moving past the FG model to simulate these nuclear effects. Benhar proposes the use of nuclear spectral functions which provided an improved description of the nucleon momentum distribution within the nucleus [32].

\section{Intranuclear Re-Scattering}

While Pauli-blocking has a dramatic effect on the apparent cross section of CC$v_{\mu}$ QE neutrino-nucleus scattering particularly at low neutrino energies and in the low $Q^{2}$ region. However final state interactions are also very important. FSIs are interactions between the struck nucleon and other nucleons as the struck nucleon passes through the nucleus. Thus for $\mathrm{CCv}_{\mu} \mathrm{QE}$ interactions FSIs change the kinematics of the final state recoil proton and also determine whether the recoil proton is re-absorbed which can lead to different final state particle multiplicities. In MINOS determination of the final state is handled by a an intranuclear cascade model via the INTRANUKE simulation within the NEUGEN MC [33].

\section{Other Nuclear Effects}

All of the neutrino generators in common use by modern experiments simulate the nucleus using a variation on the relativistic fermi gas (RFG) model. The RFG model simulates the nucleus as a collection of non-interacting nucleons that obey 


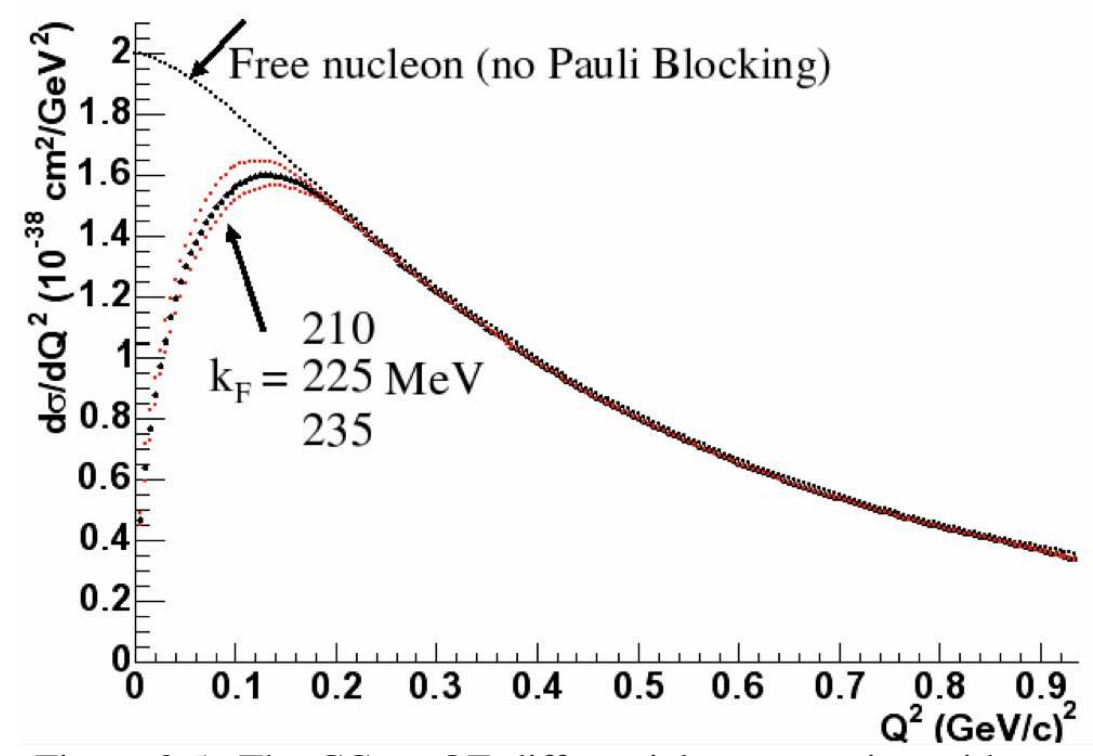

Figure 2.5: The $\mathrm{CC}-\mathrm{v}_{\mu} \mathrm{QE}$ differential cross section with respect to $Q^{2}$ with several values of $k_{F}$ compared to the free nucleon case, shows the low $Q^{2}$ suppression characteristic of Pauli-blocking. Taken from a talk given by M. Sakuda at the 2005 NuFact conference.

Fermi-Dirac statistics. The RFG is a relatively simple model with physics that is easy to calculate within an MC simulation. However the RFG model is an over simplification of the nucleus because the nucleons do interact with each other.

Measurements of the CCQE cross-section over the last ten years have revealed a 20-30\% increase in both the value of the CCQE cross section and in the measured value of $M_{A}^{Q E}$, the only free parameter in the CCQE model. The global mean for $M_{A}^{Q E}$ from early bubble chamber neutrino experiments is $1.02 \pm 0.06 \mathrm{GeV}$. Since then K2K, MiniBooNE, MINOS, and SciBooNE have all measured $M_{A}^{Q E}$ to be $20-30 \%$ higher, with only the NOMAD experiment measuring an $M_{A}^{Q E}$ that is consistent with the earlier global mean. The early experiments used deuterium as the nuclear target, while the more recent high statistics experiments have used higher A targets: carbon (MiniBooNE, SciBooNE and NOMAD), oxygen (K2K), 


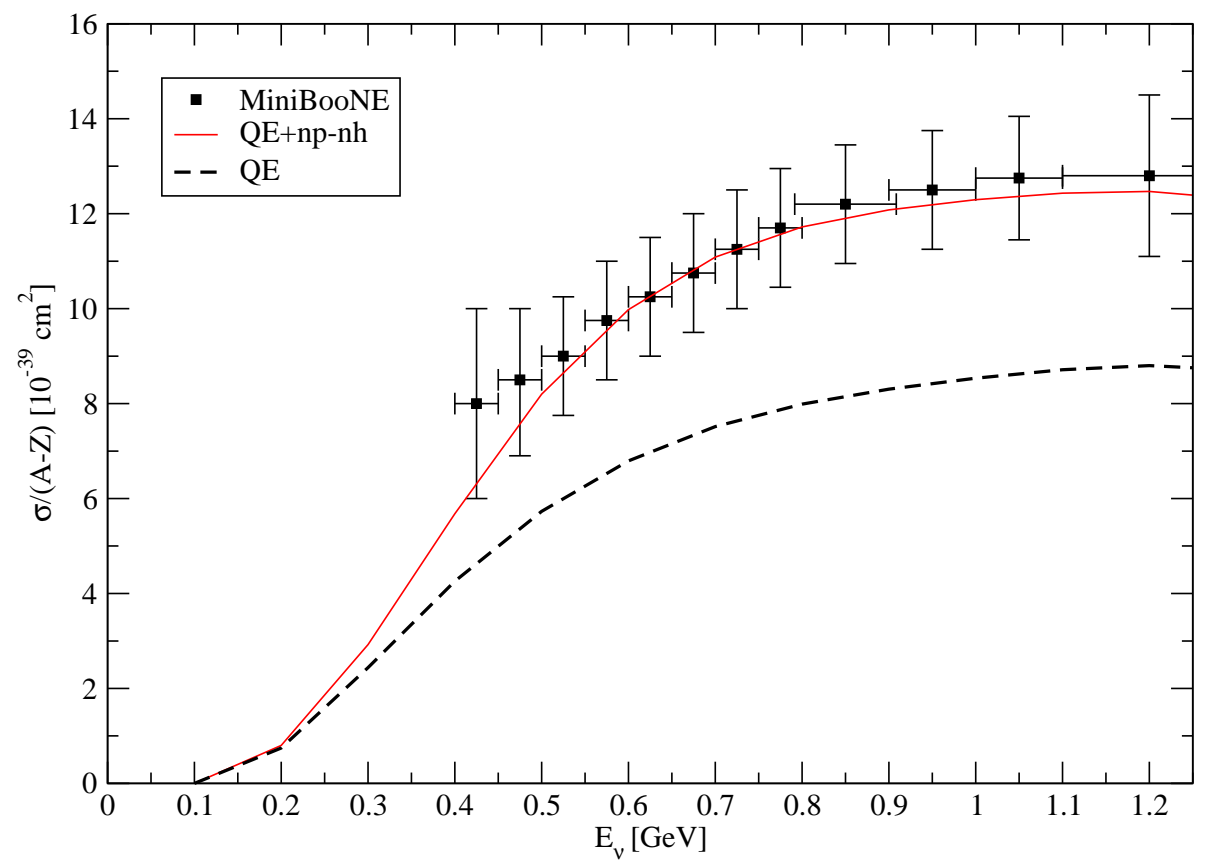

Figure 2.6: The MiniBooNE $v_{\mu}$ CCQE cross section as a function of the incident neutrino energy. The points are the MiniBooNE data, the red line is the $v_{\mu}$ CCQE cross section with an $M_{A}^{Q E}$ value of $1.02 \mathrm{GeV}$ and including the effect of the most important types of SRC interactions. The black dashed line is the same $v_{\mu}$ CCQE cross section without the SRT interactions. There is remarkable agreement between the MiniBooNE data and the $Q E+n p-n h$ model. This agreement indicates that the $20-30 \%$ discrepancy in the recent high statistics interaction experiments could, in part, be due to these SRC type interactions.

The MiniBooNE experiment has measured the $v_{\mu}$ CCQE cross section in a manner that is largely model independent [34]. This cross section measurement is consistent with an $M_{A}^{Q E}$ value of $1.35 \mathrm{GeV}$. This is more than $30 \%$ above the global mean of $1.02 \mathrm{GeV}$. The MiniBooNE detector is a spherical container of 
800 tons of mineral oil surrounded by 1280 photomultiplier tubes. MiniBooNE is a Cherenkov detector so they are largely insensitive to the recoil proton of the $v_{\mu}$ CCQE interaction. They would also be insensitive to the secondary nucleon from a CCQE-like SRC type interaction. M. Martini et al. have calculated the CCQE cross section with an $M_{A}^{Q E}$ value $1.02 \mathrm{GeV}$ and including SRC [35]. As shown in Figure 2.6 they find good agreement with the MiniBooNE data. 


\section{Chapter 3}

\section{Survey of Current Results}

Electron scattering experiments have measured the nucleon vector form factors $\left(F_{V}^{1}\right.$ and $F_{V}^{2}$ ), neutron $\beta$-decay experiments have measured the axial-vector form factor at $Q^{2}=0\left(F_{A}\left(Q^{2}=0\right)\right.$. Both of these measurements have been made with high precision. Only neutrino-nucleon scattering is sensitive to the $Q^{2}$ dependence of the axial-vector form factor $\left(F_{A}\right)$. Thus if the dipole form of the axial-vector form factor is assumed then measurements of the $\mathrm{CC}-v_{\mu} \mathrm{QE}$ cross section are equivalent to measuring the axial-vector mass $M_{A}^{Q E}$.

\subsection{Generalized Method for Measuring $M_{A}^{Q E}$}

Fundamentally the dipole assumption for the axial-vector form factor is only driven by the success of the dipole form in describing the vector form factors. There is no underlying physical theory driving the choice of the dipole form, except the success of the dipole form in describing the vector form factors. However the use of the dipole form to describe the axial-vector form factor has several advantages, most prominent of which is the use of symbolic form with a proven track record that has only one free parameter.

The axial-vector form factor is the only quantity currently not well measured within the theoretical framework describing neutrino-nucleon QE scattering (as 
given by Equation 2.45). When the axial-vector form factor is described using the dipole approximation the only free parameter is the axial-vector mass $\left(M_{A}^{Q E}\right)$. Thus the way neutrino scattering experiments measure the $M_{A}^{Q E}$ parameter is by examining (usually by fitting) the $Q^{2}$ distribution for charged current $\mathrm{QE}$ interactions.

Figure 2.4 from Section 2.2.3 shows how changes in the $M_{A}^{Q E}$ parameter results in changes to the CC- $v_{\mu}$ QE $Q^{2}$ spectrum. The upper plot in Figure 2.4 shows how changing $M_{A}^{Q E}$ changes the absolute cross section and the lower plot in Figure 2.4 shows how changing $M_{A}^{Q E}$ changes the shape of the $Q^{2}$ distribution when normalization is ignored. Thus there are two possible ways to measure the $M_{A}^{Q E}$ parameter using the $Q^{2}$ spectrum of CCQE interactions. It is possible to measure $M_{A}^{Q E}$ by examining the shape and rate information within the CCQE $Q^{2}$ spectrum, or it is also possible to measure $M_{A}^{Q E}$ just by examining the shape of the $Q^{2}$ distribution.

\subsection{Summary of World Average Values}

Table 3.1 summarizes many previous measurement of the axial-vector mass $M_{A}^{Q E}$. The weighted average for $M_{A}^{Q E}$ from neutrino-nuclei scattering experiments compiled in 2001 (deliberately chosen to exclude the modern high $Z$ target experiments) is $1.026 \pm 0.021 \mathrm{GeV}$.

When considering these measurements a number of factors are important to fully understand the differences between the measurements of the axial-vector mass $M_{A}^{Q E}$, these include but are not limited to: the nuclear target, analysis methodology, neutrino flux uncertainties, background cross section uncertainties, the formalism used in describing the form factors, and the nuclear model used. Depending on the details of how the analysis addresses all of these factors, the eventual measurement of $M_{A}^{Q E}$ could be considered as an effective measurement of the axial-vector mass, where other unknown physics are being absorbed into the measurement of the axial-vector mass. The relative importance of these factors are 
summarized below:

- Target Nucleus: The modeling uncertainty for higher $Z$ nuclear targets is largest in the low $Q^{2}$ region. Most of the previous measurement of the axialvector mass have used a low $Q^{2}$ cutoff. Most of the experiments that used a low $Q^{2}$ cutoff demonstrated consistency to small changes to this cutoff value. It isn't yet clear whether $M_{A}^{Q E}$, when treated as a effective parameter, should be a constant for all nuclei, or if it has a $\mathrm{Z}$ dependence.

- Fit Methodology: There have been several different methods used to determine the axial-vector mass used in previous analysis of $\mathrm{CC}-\mathrm{v}_{\mu} \mathrm{QE}$ data, these methods include: rate only, shape only, rate and shape, flux independent, fitting the $Q^{2}$ distribution, fitting the $Q^{2}$ distribution in slices of $E_{\mathrm{V}}$, and fitting the $Q^{2}$ and $E_{\mathrm{v}}$ distribution simultaneously. As more information is included in the fit, the analysis becomes sensitive to a larger number of uncertainties. The rate and shape method maximizes the available information, thus the analysis would become more sensitive to uncertainties in the background normalization and also uncertainties in the neutrino flux. Some previous experiments have used multiple methods, those that have usually find self-consistent values for the axial-vector mass.

- Flux Uncertainties: A significant number of the previous measurements were unable to use rate information when extracting their value of the axialvector mass because they did not have sufficient knowledge of the incident neutrino flux. These experiments would use the shape only or flux independent methods to minimize the effect that their flux uncertainty had on their measurement of the axial-vector mass. This is because the $Q^{2}$ distribution is not strongly dependent on the neutrino energy.

- Background Uncertainties: The deuterium bubble chamber experiments (such as the ANL one discussed in Section 3.3.1) were able to achieve very high QE purity, thus mostly eliminating the effect that uncertainties in the 
background cross section and $Q^{2}$ distributions had on the final axial-vector mass uncertainty. The dominant background for QE scattering is single pion production (primarily from resonant particle decay). There is a corresponding axial-vector mass for single pion production $M_{A}^{R E S}$ (also called $M_{A}^{1 \pi}$ ). When the QE selection has a lower purity of QE interactions the extracted $M_{A}^{Q E}$ value could have an artificially high or low value due to this background contamination. Also correlations between $M_{A}^{Q E}$ and $M_{A}^{R E S}$ will have the effect of increasing the uncertainty in best fit QE axial-vector mass.

- Vector Form Factors: Previous measurements of the axial-vector mass have assumed the dipole form for the nucleon weak vector form factors. However, recent work by Bodek et al [36] [37] suggests an alternate form for these form factors. Because the extraction of the axial-vector mass is sensitive to the choice of these vector form factors, and also to other parameters used in model of the nucleon ( $\operatorname{such}$ as $F_{A}\left(Q^{2}=0\right)$ ), the choice of global average could have an effect on the extracted axial-vector mass.

- Nuclear Model Used: Most neutrino experiments are using or have used the RFG model to describe the behavior of the nucleus. It seems pretty clear now that the RFG model is inadequate to describe the actual behavior of the nucleus. Newer neutrino event generator MC (such as GENIE [38]) are beginning to included improved models of Pauli-blocking such as nuclear spectral functions. The treatment of Pauli-blocking is not the only element missing from neutrino event generators, as discussed in Sections 3.4, and 2.2.4, there are other nuclear effects due to correlations and interactions between nucleons that are not considered in neutrino-nuclei scattering, but appear to be more important that previously thought. 


\begin{tabular}{|c|c|c|c|c|c|}
\hline Experiment & Target & Events & Method & $M_{A}^{Q E} \mathrm{GeV}$ & Ref. \\
\hline ANL 69 & Steel & & Shape & $1.05 \pm 0.20$ & [39] \\
\hline ANL 73 & Deuterium & 166 & $\begin{array}{l}\text { Cross Section } \\
\text { Shape } \\
\text { Rate and Shape }\end{array}$ & $\begin{array}{l}0.97 \pm 0.16 \\
0.94 \pm 0.18 \\
0.95 \pm 0.12\end{array}$ & [40] \\
\hline ANL 77 & Deuterium & $\sim 600$ & $\begin{array}{l}\text { Cross Section } \\
\text { Shape } \\
\text { Rate and Shape }\end{array}$ & $\begin{array}{r}0.75_{-0.11}^{+0.13} \\
1.01 \pm 0.09 \\
0.95 \pm 0.09\end{array}$ & [41] \\
\hline $\begin{array}{l}\text { ANL } 82 \\
\text { (See Section 3.3.1) }\end{array}$ & Deuterium & 1737 & $\begin{array}{l}\text { Cross Section } \\
\text { Shape } \\
\text { Rate and Shape }\end{array}$ & $\begin{array}{l}0.74 \pm 0.12 \\
1.05 \pm 0.05 \\
1.03 \pm 0.05\end{array}$ & [42] \\
\hline BNL 81 & Deuterium & 1138 & Shape & $1.07 \pm 0.06$ & [43] \\
\hline BNL 90 & Deuterium & 2538 & Shape & $1.070_{-0.045}^{+0.040}$ & [44] \\
\hline FNAL 81 & Deuterium & 362 & Shape & $1.07 \pm 0.06$ & [45] \\
\hline NuTeV 04 & Steel & 21614 & Cross Section & $1.11 \pm 0.08$ & [46] \\
\hline $\begin{array}{l}\text { MiniBooNE } \\
\text { (See Section 3.3.3) }\end{array}$ & Mineral Oil & 193709 & $\begin{array}{l}\text { Shape } \\
\text { Rate and Shape }\end{array}$ & $\begin{array}{l}1.23 \pm 0.20 \\
1.35 \pm 0.17 \\
\end{array}$ & $\begin{array}{l}{[31]} \\
{[34]}\end{array}$ \\
\hline CERN HLBC 64 & Freon & 236 & Shape & $\begin{array}{l}1.00_{-0.20}^{+0.35} \\
\end{array}$ & [47] \\
\hline CERN HLBC 69 & Propane & 130 & Rate and Shape & $0.70 \pm 0.20$ & [48] \\
\hline CERN GGM 77 & Freon & 687 & $\begin{array}{l}\text { Shape } \\
\text { Rate }\end{array}$ & $\begin{array}{l}0.96 \pm 0.16 \\
0.88 \pm 0.19\end{array}$ & [49] \\
\hline CERN GGM 79 & Propane/Freon & 556 & $\begin{array}{l}\text { Shape } \\
\text { Rate }\end{array}$ & $\begin{array}{l}0.99 \pm 0.12 \\
0.87 \pm 0.18\end{array}$ & {$[50]$} \\
\hline CERN BEBC 90 & Deuterium & 552 & $\begin{array}{l}\text { Shape } \\
\text { Rate }\end{array}$ & $\begin{array}{l}1.08 \pm 0.08 \\
0.94 \pm 0.07\end{array}$ & $\begin{array}{l}51] \\
\end{array}$ \\
\hline $\begin{array}{l}\text { NOMAD } \\
\text { (See Section 3.3.2) }\end{array}$ & Argon/Ethane & 14021 & Rate and Shape & $\begin{array}{r}1.05 \pm 0.02 \\
\pm 0.06\end{array}$ & {$[52]$} \\
\hline IHEP 82 & Aluminum & 898 & Shape & $1.00 \pm 0.007$ & {$[53]$} \\
\hline IHEP 85 & Aluminum & 1753 & Shape $(v+\bar{v})$ & $1.00 \pm 0.04$ & {$[54]$} \\
\hline IHEP SCAT 88 & Freon & 464 & Rate and Shape & $0.96 \pm 0.15$ & {$[55]$} \\
\hline IHEP SCAT 90 & Freon & & $\begin{array}{l}\text { Rate } \\
\text { Shape } \\
\text { Rate and Shape }\end{array}$ & $\begin{array}{l}1.08 \pm 0.07 \\
1.05 \pm 0.07 \\
1.06 \pm 0.05\end{array}$ & {$[56]$} \\
\hline K2K 06, SciFi & Water & $\sim 12000$ & Shape & $1.20 \pm 0.12$ & [57] \\
\hline K2K 08, SciBar & Carbon & & Shape & $1.144 \pm 0.077$ & [58] \\
\hline
\end{tabular}

Table 3.1: Summary of some $M_{A}^{Q E}$ measurements. Table reproduced from [52]. 


\subsection{Survey of $M_{A}^{Q E}$ Measurements}

Table 3.1 summarizes measured results for the quasi-elastic axial-vector mass $M_{A}^{Q E}$. What follows will be a more detailed look at a few of these publications. These results are presented as 'case-studies' of the different ways that $M_{A}^{Q E}$ can be measured, while also exploring sources of some of the tension that is seen between the earlier measurements and the more recent measurements.

\subsubsection{Argonne 12-Foot Bubble Chamber}

Argonne National Lab (ANL) has the honor of having made the most accurate measurement of $M_{A}^{Q E}$. This measurement was made using the twelve foot bubble chamber at ANL, which was exposed to a neutrino beam produced at the Zero Gradient Synchrotron (ZGS) facility. The ZGS focused $12.4 \mathrm{GeV}$ protons onto a beryllium target, the resulting hadrons were focused by two magnetic horns. The focused hadrons (mainly $\pi^{+}$) were allowed to decay to into $\mu^{+}$and $v_{\mu}$, any un-decayed hadrons and the charged leptons where absorbed by the steel and lead absorber at the end of the $30 \mathrm{~m}$ decay region. The remaining muon neutrinos would pass through the absorber, this resulting beam of neutrinos were incident on the deuterium filled bubble chamber.

The resulting muon neutrino beam from the ZGS facility had a peak neutrino energy of $\sim 0.5 \mathrm{GeV}$ with a tail that reached up to $\sim 6 \mathrm{GeV}$. This neutrino beam was modeled using a simulation of the neutrino beamline, the simulation was tuned using the measured hadron production from $p$-Be scattering. This constrained the flux to an estimated uncertainty of $\pm 15 \%$ except in the high energy tail where a significant fraction of the neutrino production came from kaon decays, here the lack of $\mathrm{K}^{+}$production measurements increased the flux uncertainty to $\pm 25 \%$.

The Argonne twelve foot bubble chamber was filed with liquid deuterium that had been heated to just below the boiling point for deuterium. The pressure within the bubble chamber was mechanically reduced in anticipation of particles passing through the chamber. This lower pressure causes the deuterium to en- 
ter a metastable superheated phase, thus when charged particles enter the chamber the would leave an ionization trail. The ionization would cause bubbles to form around the ionization path (hence 'bubble' chamber). As the pressure in the chamber was continued to be reduced, the bubbles would expand until they were big enough to be seen by an array of cameras which would photograph the bubble chamber activity from multiple angles. The chamber was positioned inside a solenoidal magnetic field thus the particles would travel in a helical path. Since the bubble density around the particle's path is proportional to the particle's energy loss it was possible to tag individual particle types (through energy loss) and the particle's momentum (through through the helical path and the Lorenz force law). The final analysis on the twelve foot bubble chamber neutrino data used $2.4 \times 10^{6}$ photographs of the bubble chamber activity.

The analysis was done by having physicists visually scan the photographs of the bubble chamber, who would flag the interesting one, two, and three prong events. Every photograph was scanned by at least two different physicists (some were scanned by three). Using the information from multiple scanners the scanning efficiency was estimated to be $98 \pm 2 \%$ for fiducial events. Because the target of the incident neutrino was deuterium, after the neutrino struck the nuclei it was also possible to see the nuclear remnant proton (called the spectator proton because it doesn't participate in the fundamental interaction). Thus the scanners looked for three prong events along with one and two prong events. All of the two and three prong events underwent geometric reconstruction and kinematic fitting to the QE hypothesis ${ }^{1}$. This lead to a final background estimate of $2 \pm 2 \%$ and the final analysis used 1737 event photographs with an estimated background of $35 \pm 35$ events [42].

\footnotetext{
${ }^{1}$ Because the QE interaction is a simple two body interaction (two particles in the initial and final state) the kinematics of the QE interaction are constrained such that only the measured information about the outgoing lepton are needed to determine the incident neutrino energy. This constraint means that the muon kinematics plus the neutrino beam direction can be used to predict the energy and momentum of the final state recoil proton. The reconstructed momentum of the recoil proton can be compared to the predicted momentum of the recoil proton, disagreements can be used to reject background to $\mathrm{QE}$ signal
} 
The $Q^{2}$ distribution for the $\mathrm{QE}$ selected event sample was fit using the axialvector dipole description of the form factors from Chapter 2. This involved making all of the same assumptions that went into the derivations of the $\mathrm{QE}$ differential cross section; assumptions such as, the CVC hypothesis, time-reversal symmetry, charge symmetry, etc. Nuclear effects were taken into account as well through the use of a correction function that changed as a function of $Q^{2}$, though these effects were not particularly strong given that the neutrino target was deuterium.

The analysis used several different methods to extract the $M_{A}^{Q E}$ parameter: rate-only, shape-only, rate and shape, and a flux independent method. Each of these analysis methods used a different likelihood function. These likelihood functions are shown in Equations 3.1 to 3.4:

$$
\begin{gathered}
\mathcal{L}_{\text {rate }}=-\ln \left(2^{\frac{1}{2}} N_{\text {data }}^{\frac{1}{2}} \sigma_{N}\right)-\frac{1}{2}\left(\frac{N_{\text {data }}-N_{\text {theory }}}{\sigma_{n}}\right)^{2} \\
\mathcal{L}_{\text {shape }}=\sum_{i=1}^{N_{\text {data }}} W\left(Q_{i}^{2}\right)\left[\frac{\frac{d \sigma}{d Q^{2}}\left(Q_{i}^{2}, E_{\mathrm{v} i} ; M_{A}^{Q E}, M_{V}\right) R\left(Q_{i}^{2}\right) \Phi\left(E_{\mathrm{v} i}\right)}{\iint \frac{d \sigma}{d Q^{2}}\left(Q_{i}^{2}, E_{\mathrm{v} i} ; M_{A}^{Q E}, M_{V}\right) R\left(Q^{2}\right) \Phi\left(E_{\mathrm{v} i}\right) d Q^{2} d E_{\mathrm{v}}}\right] \\
\mathcal{L}_{\text {total }}=\mathcal{L}_{\text {rate }}+\mathcal{L}_{\text {shape }} \\
\mathcal{L}_{F I}=\sum_{i=1}^{N_{\text {data }}} W\left(Q_{i}^{2}\right)\left[\frac{\frac{d \sigma}{d Q^{2}}\left(Q_{i}^{2}, E_{\mathrm{v} i} ; M_{A}^{Q E}, M_{V}\right) R\left(Q_{i}^{2}\right)}{\int \frac{d \sigma}{d Q^{2}}\left(Q_{i}^{2}, E_{\mathrm{v} i} ; M_{A}^{Q E}, M_{V}\right) R\left(Q^{2}\right) d Q^{2}}\right]
\end{gathered}
$$

where $W\left(Q^{2}\right)$ is the weight due to scanning efficiency, $R\left(Q_{i}^{2}\right)$ is the correction factor accounting for nuclear effects, $\Phi\left(E_{v i}\right)$ is the neutrino flux, and $\frac{d \sigma}{d Q^{2}}$ is the differential cross section. The results from [42] are shown in Table 3.2.

Figure 3.1 shows the $Q^{2}$ distribution of the QE selected events from [42], also contains the theoretical prediction based on Equation 2.45 using the best fit $M_{A}^{Q E}$ value from the flux independent likelihood analysis. This fit was only performed on events with $Q^{2}>0.05 \mathrm{GeV}^{2}$. 


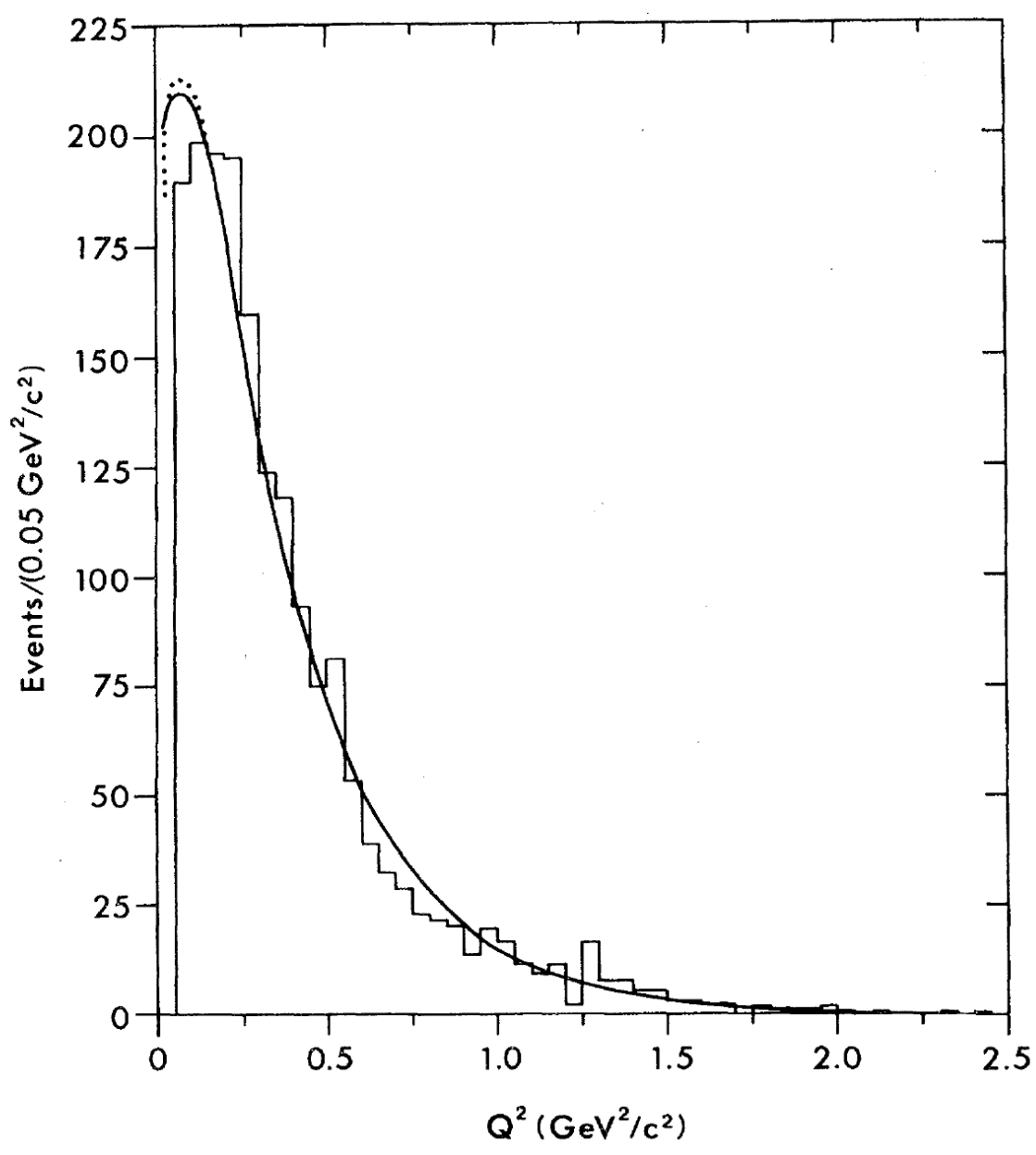

Figure 3.1: $Q^{2}$ distribution from [42]. The data corresponds to the shown histogram, the solid curve corresponds to the dipole axialvector form factor utilizing the best fit from the flux independent analysis from Table 3.2. The dotted curve corresponds to an alternate form of the axial-vector form factor with $M_{A}^{Q E}=1.11 \mathrm{GeV}$. 


\begin{tabular}{|c|c|}
\hline Analysis & $M_{A}^{Q E}(\mathrm{GeV})$ \\
\hline \hline Rate & $0.74 \pm 0.12$ \\
\hline Shape & $1.05 \pm 0.05$ \\
\hline Rate + Shape & $1.03 \pm 0.05$ \\
\hline Flux Independent & $1.00 \pm 0.05$ \\
\hline
\end{tabular}

Table 3.2: Best fit results for $M_{A}^{Q E}$ using the likelihood functions given in Equations 3.1 to 3.4 as discussed in [42].

\subsubsection{Results From the NOMAD Experiment}

The NOMAD experiment used neutrinos generated by the CERN $450 \mathrm{GeV}$ proton synchrotron (SPS). Protons were taken from the SPS and allowed to interact with a beryllium target, the resulting hadrons (primarily pions and kaons) were then focused using a magnetic horn. The focused beam of pions and kaons produced a beam of predominantly muon neutrinos as a result of further decays. The resulting neutrino flux spanned $O(1) \leq E_{\mathrm{v}} \leq 300 \mathrm{GeV}$, with a peak flux at $\sim 17 \mathrm{GeV}$ and a mean energy of $24.3 \mathrm{GeV}$ [59].

The NOMAD detector consisted of 44 drift chambers filled with an argonethane gas mixture as an active target with a total fiducial mass of 2.7 tons, the main detector region is located within a $0.4 \mathrm{~T}$ magnetic field. The average density of the gas in the drift chambers was $0.1 \mathrm{~g} / \mathrm{cm}^{3}$ and provided an overall track reconstruction efficiency of $95 \%$. Particle identification was provided by the transition radiation detector (TRD) placed after the main drift chambers of the detector.

NOMAD constrains the overall normalization of their flux by examining the high energy of their neutrino spectrum which is dominated by deep inelastic scattering. This theoretically and experimentally well known cross section $(\sigma)$ is used to extract the flux $(\Phi)$ from the measured event rate $\left(N_{\text {events }}\right)$, where $N_{\text {events }}=\Phi \sigma$. then projecting the flux into the regions with less well known cross sections.

NOMAD's CCQE analysis [52] uses two separate and exclusive event selections both of which are dominated by CCQE interactions. These event samples 
are; A one track event selection where only the muon track is visible, and a two track event selection where the muon and the recoil proton are both visible. The two track selection has a proton momentum threshold of $300 \mathrm{MeV} / \mathrm{c}$ if the momentum of the candidate second track does not exceed this value than the event is still classified as a one track event. NOMAD extracted the CCQE cross section from both of these selections separately as well as in a combined manner, shown in Figure 3.2. The nuclear formation time $\left(\tau_{0}\right)$ is a major systematic that causes event navigation between the one and two track event se-

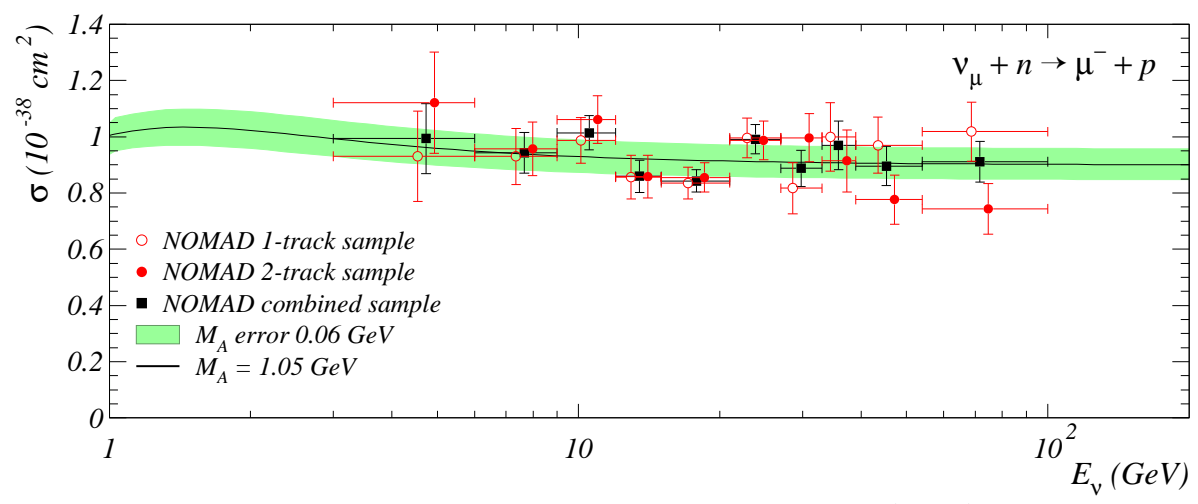

Figure 3.2: From [52]. Comparison the NOMAD $\left\langle\sigma_{q e l}\right\rangle_{v_{\mu}}$ measurements as a function of the neutrino energy in the 1-track and 2-track subsamples.

lections. This event migration causes a strong correlation between $\tau_{0}$ and $M_{A}^{Q E}$ when the axial-vector mass is extracted from the one track selection and an anticorrelation between $\tau_{0}$ and $M_{A}^{Q E}$ when the axial-vector mass is extracted from the two track selection. This correlation is broken when the $M_{A}^{Q E}$ extraction is done on both selections simultaneously. This extracted best fit axial-vector mass value is $M_{A}^{Q E}=1.05 \pm 0.02($ stat $) \pm 0.06($ syst $) \mathrm{GeV}$. 


\subsubsection{Results From the MiniBooNE Experiment}

The MiniBooNE experiment has published two separate papers on CCQE scattering results: [31], and [34]. These publications documented two separate analyses that extracted different (though consistent) values of the the $M_{A}^{Q E}$ parameter. The first publication [31] presented a simple shape-only fit using the MiniBooNE MC that attempted to parameterize the low $Q^{2}$ region by fitting for a Pauli blocking parameter simultaneously. The second publication [34] produced a axial-vector mass measurement as a side product to a more inclusive model independent crosssection and double differential cross section measurement of the detector observables.

The MiniBooNE experiment at Fermi National Accelerator Lab (FNAL) measures a beam of muon neutrinos incident on 818 tons of mineral oil $\left(\mathrm{CH}_{5}\right)$ contained within a spherical detector of radius of 6.1 meters and surrounded by 1280 8in photo multiplier tubes (PMTs) which capture the Cherenkov light as charged particles pass through the detector. The FNAL booster provides protons accelerated to $8 \mathrm{GeV}$ kinetic energy. The protons from the booster beam scatter off of a beryllium target located inside a magnetic horn. The magnetic field provided by the horn focuses primarily $\pi^{+}$but also focuses some $K^{+}$. These hadrons will then decay to anti-muons and muon neutrinos. Most of the anti-muons are absorbed by the earth between the booster beam and the MiniBooNE detector leaving just a beam of muon neutrinos. The booster generates a neutrino beam with a mean neutrino energy of $0.7 \mathrm{GeV}$ and has an energy distribution such that $99 \%$ of the neutrinos have energy less than $2.5 \mathrm{GeV}$.

MiniBooNE identifies QE interaction candidates by looking for a lone muon with no secondary hadron. MiniBooNE identifies muons by looking for the muon ring and the secondary Michel electron ring from the decay of the muon. Looking for the Michel electron helps to distinguish the muon ring from a potential pion ring from an NC interaction where there will be two decay electrons from the pion decay chain. This selection consisted of 193709 selected events, the estimated efficiency was $35 \%$ with an estimated purity of $74 \%$. 


\section{The MiniBooNE Axial-Vector Mass Measurement}

The MiniBooNE extraction of the axial-vector mass proceeds in a manner that is similar to the way the ANL bubble chamber experiment measured $M_{A}^{Q E}$, by fitting the $Q^{2}$ distribution of CC- $v_{\mu} \mathrm{QE}$ interactions for the axial-vector mass parameter of the dipole form factor. The MiniBooNE experiment uses a Relativistic Fermi Gas (RFG) model within their neutrino interaction simulation to model the scattering of neutrinos off of carbon nuclei. It was necessary to fully understand any sources of differences in the spectrum due to the interaction model for the purposes of the MiniBooNE oscillation analysis [60], thus MiniBooNE attempted to parameterize the low $Q^{2}$ region of their $Q^{2}$ spectrum where nuclear effects are expected to dominate. MiniBooNE did this by introducing an additional parameter, $\kappa$, that changes the amount of Pauli-blocking in a QE interaction. MiniBooNE fits the shape of the $Q^{2}$ distribution all the down to $Q^{2}=0 \mathrm{GeV}^{2}$, from this fit MiniBooNE obtains the best fit values of: $M_{A}^{Q E}=1.23 \pm 0.20 \mathrm{GeV}$ for the axial-vector mass, and $\kappa=1.019 \pm 0.011$. Figure 3.3 from [31] shows the data and best fit MC $Q^{2}$ distribution, along with a $1 \sigma$ contour of $M_{A}^{Q E}$ vs. $\kappa$.

\section{The MiniBooNE QE Cross Section Measurement}

Later MiniBooNE attempted a more sophisticated model independent measurement of the CC- $v_{\mu}$ QE double differential cross section with respect to the detector observables: muon kinetic energy $\left(T_{\mu}\right)$, and the muon scattering angle $\left(\theta_{\mu}\right)$. Because this analysis measured a cross section it was necessary to perform several additional analysis steps that were not done in the MiniBooNE axial-vector mass measurement. These additional steps included a correction to the distribution based on the efficiency as a function of observables $\left(T_{\mu}\right.$ and $\left.\theta_{\mu}\right)$, and a subtraction of the non-CCQE background.

MiniBooNE does an in situ measurement of the non-CCQE background. The in situ measurement is done by identifying an event sample that is dominated by non-CCQE interactions. The MiniBooNE non-CCQE event selection looks for 


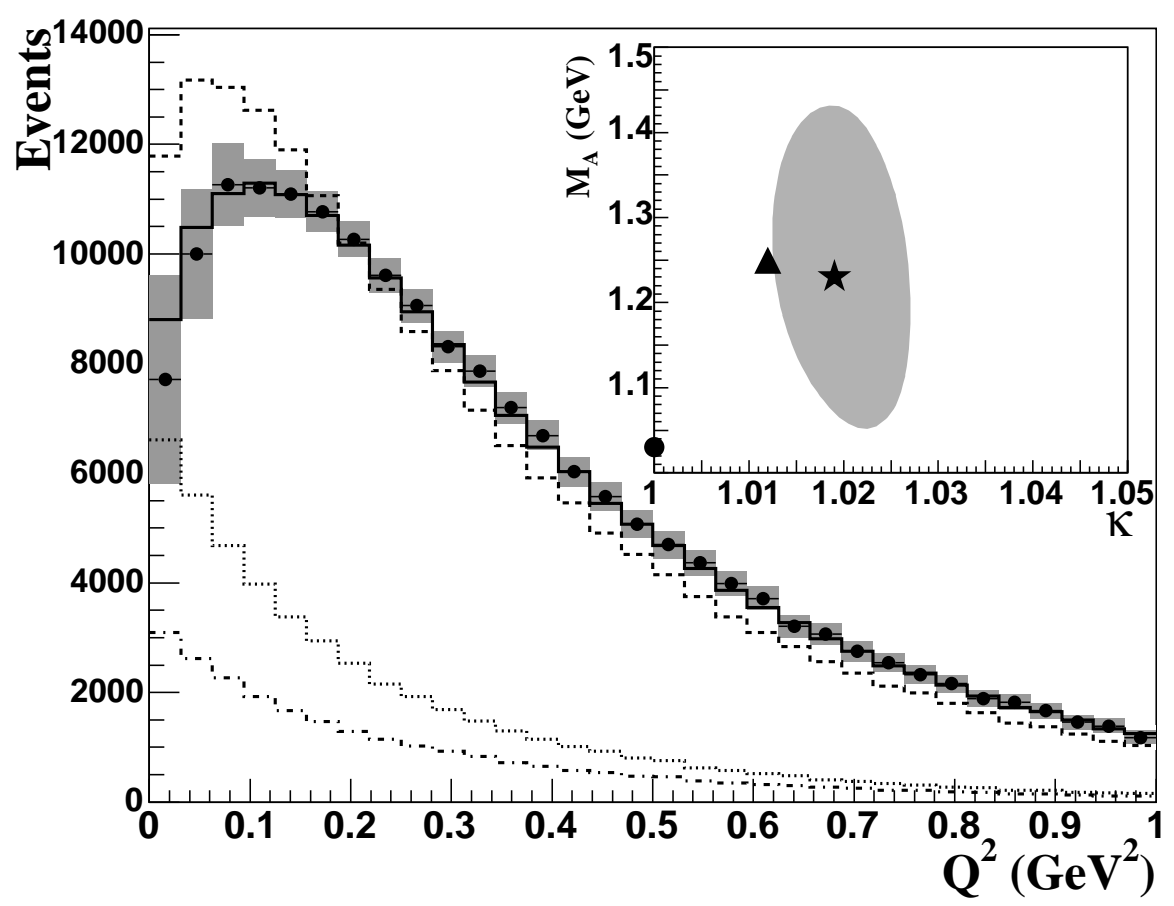

Figure 3.3: From [31]. Reconstructed $Q^{2}$ for $v_{\mu}$ CCQE events including systematic errors. The simulation, before (dashed) and after (solid) the fit, is normalized to data. The dotted (dot-dash) curve shows backgrounds that are not CCQE (not "CCQE-like"). The inset shows the $1 \sigma \mathrm{CL}$ contour for the best-fit parameters (star), along with the starting values (circle), and fit results after varying the background shape (triangle).

an additional (third) Cherenkov ring outside of the first two Cherenkov rings of the CCQE selection. This non-CCQE event selection is composed of $90 \%$ events with a single muon plus single pion in the final state. Muon plus single pion are the dominant background to the CCQE selected events. The CC1 $\pi$ MC event sample is then reweighted such that the MC reconstructed $Q^{2}$ distribution matches the data reconstructed $Q^{2}$ distribution. The $\mathrm{MC} C \mathrm{CC} 1 \pi$ correction function is then applied to the MC CC1 $\pi$ background to the CCQE MC event sample, then an equal number of events are subtracted from the CCQE data event sample. This 

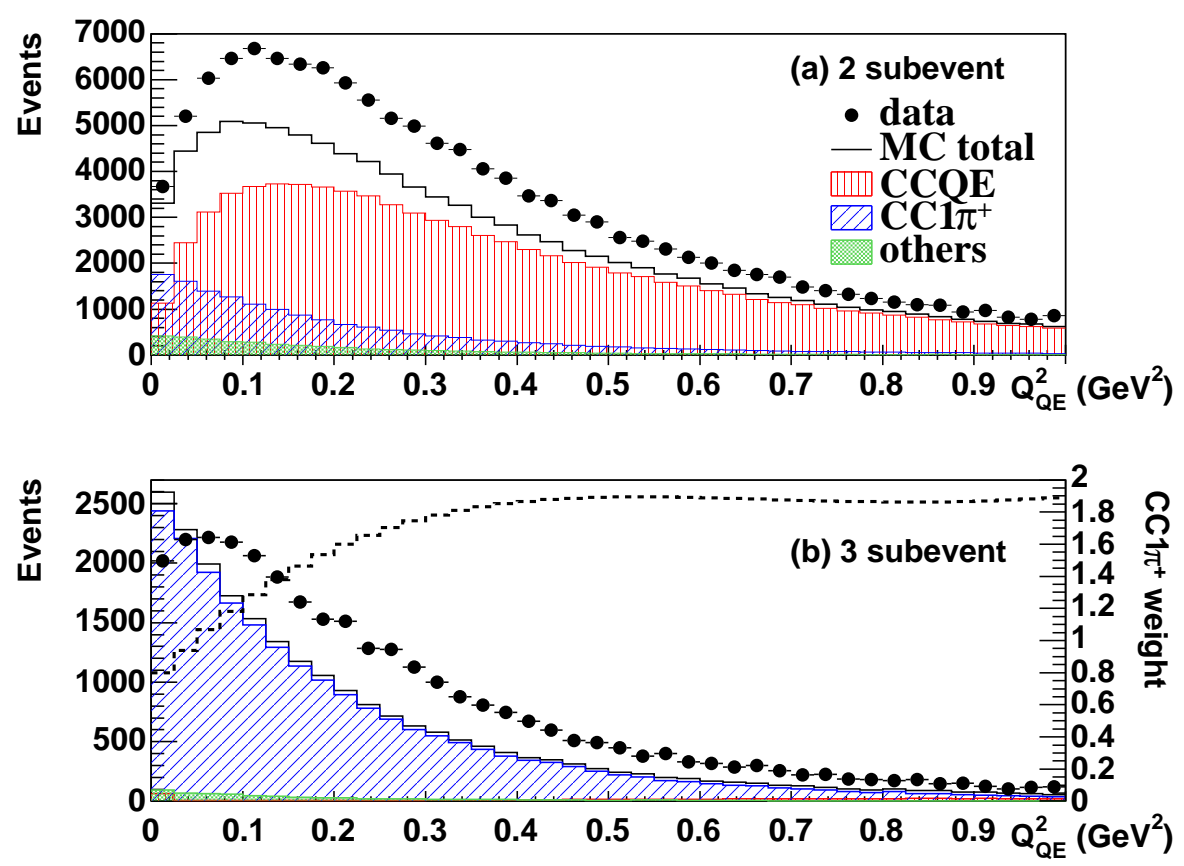

Figure 3.4: From [34]. Distribution of events in $Q^{2} \mathrm{QE}$ for the (a) 2 and (b) 3 subevent samples before the application of the $\mathrm{CC} 1$ background correction. Data and MC samples are shown along with the individual MC contributions from CCQE, CC1 $\pi$, and other channels. In (b), the dashed line shows the $\mathrm{CC} 1 \pi$ reweighting function (with the $y$-axis scale on the right) as determined from the background $t$ procedure.

gives an approximately pure sample of CCQE events. The CCQE and CC1 $\pi$ event samples are shown in Figure 3.4.

MiniBooNE uses the background subtracted (and efficiency corrected) CCQE event sample to make measurements of the cross section as function of several different kinematic variables. They also, as a separate measurement, perform a fit of the $Q^{2}$ distribution using the background subtracted data and MC, CCQE selected events for the axial-vector mass and the $\kappa$ parameter. This fit produces best fit values of $M_{A}^{Q E}=1.35 \pm 0.17 \mathrm{GeV}$ and $\kappa=1.007 \pm 0.012$. This axial- 

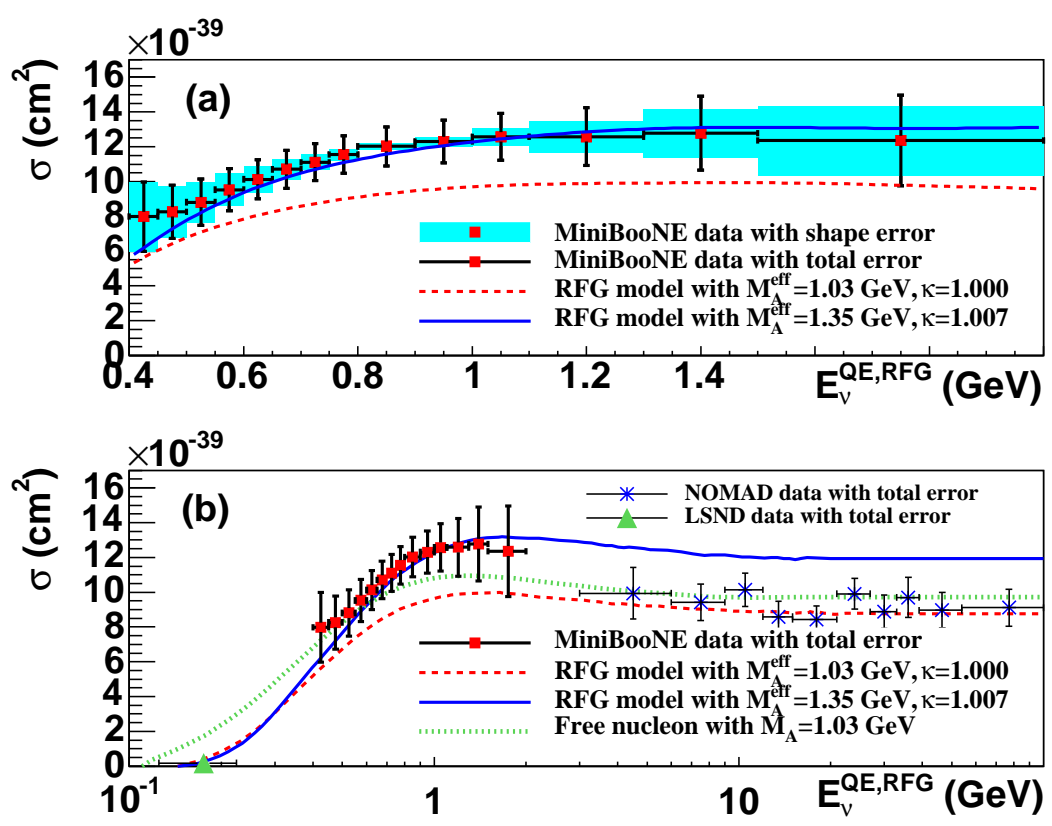

Figure 3.5: From [34]. Flux-unfolded MiniBooNE $v_{\mu}$ CCQE cross section per neutron as a function of neutrino energy. In (a), shape errors are shown as shaded boxes along with the total errors as bars. In (b), a larger energy range is shown along with results from the LSND [61] and NOMAD [52] experiments. Also shown are predictions from the NUANCE simulation for an RFG model with two different parameter variations and for scattering from free nucleons with the world-average MA value. Numerical values are provided in Table $\mathrm{X}$ in the appendix (of [34]).

vector mass value is consistent with previous higher $N$ nuclear target neutrino experiments such as the previous MiniBooNE measurement [31], the $\mathrm{K} 2 \mathrm{~K}$ axial mass measurement [57] and others (see Table 3.1). The best fit value for $\kappa$ is consistent with the standard RFG model where $\kappa$ equals 1.00 . The cross section as a function of neutrino energy is shown in Figure 3.5 this plot also shows the NOMAD measured cross section as function of neutrino energy and the RFG theoretical prediction for $M_{A}^{Q E}=1.35, \kappa=1.007$, and also for $M_{A}^{Q E}=1.03$ and 
$\kappa=1.000$.

\subsection{Resolving the MiniBooNE-NOMAD Discrepancy}

Figure 3.5(b) shows the neutrino cross section as a function of energy from two separate experiments (MiniBooNE and NOMAD) performed at two very different neutrino energies. Figure 3.5(b) also shows the theoretical prediction for the best fit values from each of these experiments. It is difficult to reconcile these two experimental results using the formalism of Llewellyn-Smith and the RFG model. Experiments such as the ANL twelve foot bubble chamber have well measured the axial-vector mass on deuterium, it seems unlikely that the Llewellyn-Smith formalism is significantly wrong. Thus the likely contributor would appear to be the nuclear model implemented in most neutrino scattering experiments, the RFG model.

The RFG model assumes that the nucleons bound within the nucleus do not interact with the other nucleons within the nucleus. This is clearly not the case, however it was believed that the contribution from nucleon-nucleon interactions was small enough to ignore for the purposes of neutrino-nucleus scattering. There is an ever increasing body of evidence that these nucleon-nucleon interactions are important for neutrino-nucleus scattering.

The MiniBooNE and NOMAD results, taken together, require that the effect these nucleon-nucleon interactions have on neutrino-nucleus scattering must be neutrino energy dependent. There is a strong enhancement of the neutrino-nucleus cross section at the low neutrino energies of MiniBooNE. There is no detectable effect at the higher neutrino energies of NOMAD. Meson Exchange Currents (MECs) can explain the apparent energy dependence of the MiniBooNE/NOMADanomaly. MECs result in an enhancement of the transverse (high $\theta_{\mu}$ ) component of the cross section without a corresponding enhancement in the longitudinal (low $\theta_{\mu}$ ) component of the cross section. Another consequence of MECs is a stronger enhancement of the neutrino-nucleus scattering cross section than the 

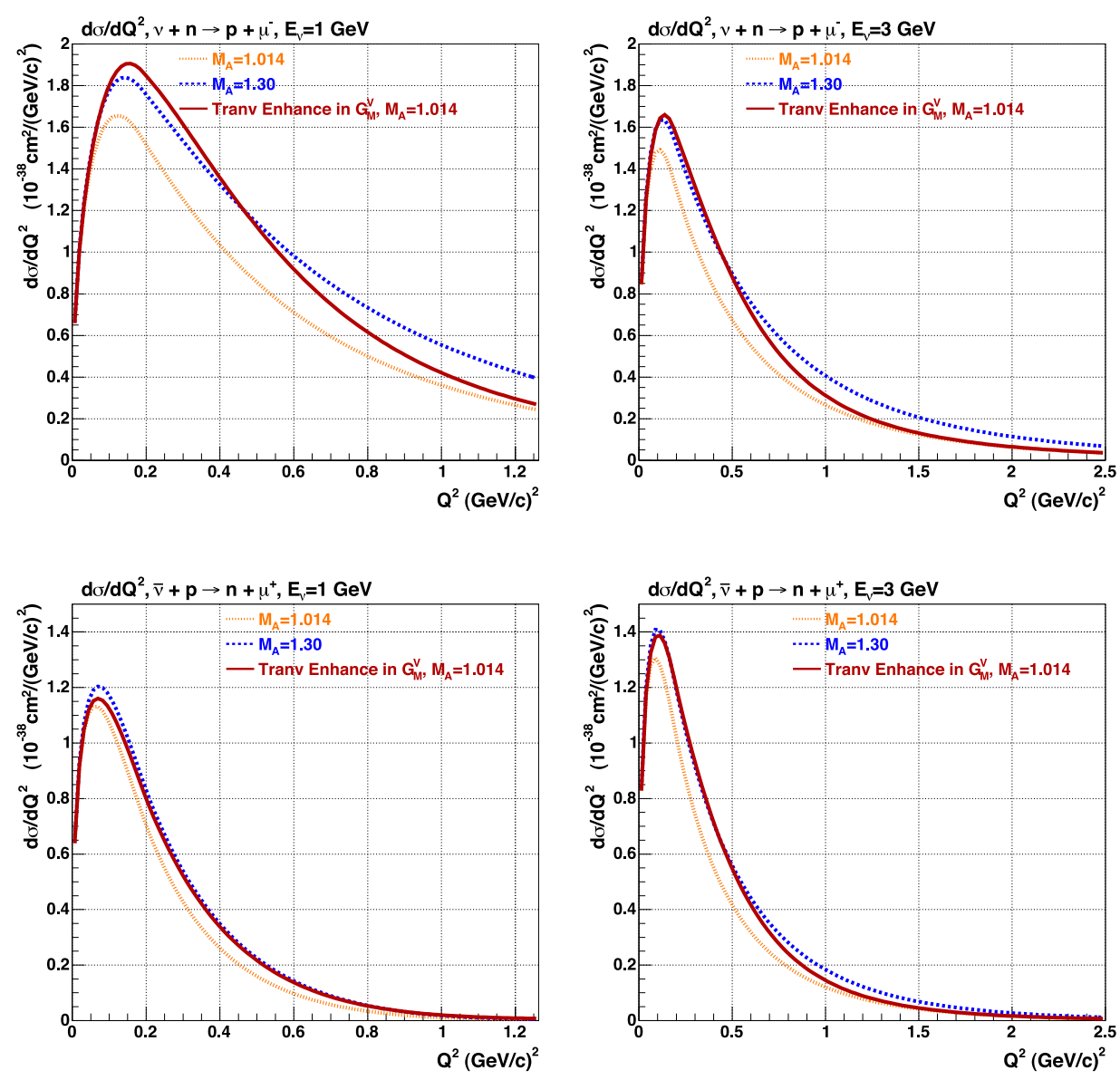

Figure 3.6: From [62]. Left: The QE differential cross section $\left(\mathrm{d} \sigma / \mathrm{d} Q^{2}\right)$ as a function of $Q^{2}$ for $\mathrm{v}_{\mu}, \overline{\mathrm{v}}_{\mu}$ energies of $1.0 \mathrm{GeV}$ (maximum accessible $\left.Q_{\max }^{2}=1.3(\mathrm{GeV} / \mathrm{c})^{2}\right)$. Here, the orange dotted line is the prediction of the "Independent Nucleon $\left(M_{A}=1.014\right)$ " model. The blue dashed line is the prediction of the the "Larger $M_{A}\left(M_{A}=1.3\right)$ " model. The red line is prediction of the "Transverse Enhancement" model. Top (a): $v_{\mu}$ differential QE cross sections. Bottom (b): $\overline{\mathrm{v}}_{\mu}$ differential QE cross sections. Right: Same as Left for $v_{\mu}, \bar{v}_{\mu}$ energies of $3.0 \mathrm{GeV}$ (maximum accessible $Q_{\max }^{2}=$ $\left.4.9(\mathrm{GeV} / \mathrm{c})^{2}\right)$. 
antineutrino-nucleus scattering cross section. As shown in Figure 3.6 from [62] the transversely enhanced neutrino-carbon differential cross section of $1 \mathrm{GeV}$ neutrinos is $\sim 25 \%$ larger than the neutrino-nulceon differential cross section of $1 \mathrm{GeV}$ neutrinos. While the transversely enhanced antineutrino-carbon cross section is $\sim 5 \%$ larger than the neutrino-nucleon cross section. This trend is smaller for the $3 \mathrm{GeV}$ neutrinos/antineutrinos.

The other possibility is that this is not a neutrino energy effect but is instead because of final state identification. The MiniBooNE QE identification procedure does not use any information about the presence of the recoil proton in the final state, because the MiniBooNE detector is very insensitive to the presence of the recoil proton. This is contrasted with the NOMAD experiment which is very good at identifying the presence of final state particles. NOMAD has a proton momentum threshold of $300 \mathrm{MeV} / c$; above this threshold the event is classified as a candidate two track event, below this threshold the event is classified as a one track event. It is possible that this discrepancy in final state identification is responsible for the discrepancy in the measured axial-vector mass values. This would happen if NOMAD rejected as being not QE like interactions that have additional particles in the final state that would appear as a result of the nucleonnucleon interactions, while MiniBooNE would not be able to distinguish these interactions from QE interactions. 


\section{Chapter 4}

\section{MINOS Experiment}

The Main Injector Neutrino Oscillation Search (MINOS) experiment [63] uses two nearly identical neutrino detectors positioned $734 \mathrm{~km}$ away from each other. The two detectors measure the energy spectrum of the neutrino beam produced by the Neutrinos at the Main Injector (NuMI) facility at FNAL [64] with a baseline of $734 \mathrm{~km}$. The neutrinos pass through the Near Detector (ND) approximately $1 \mathrm{~km}$ from the NuMI production target. The neutrinos then pass through $734 \mathrm{~km}$ of earth before reaching the Far Detector (FD) at the end of the baseline. The general geographic layout of the MINOS experiment is shown in Figure 4.1. For more details about the MINOS experimental configuration see [65].

\subsection{The NuMI Beamline}

The first stage of proton acceleration is the FNAL linear accelerator (Linac) which accelerates the protons up to $400 \mathrm{MeV} / \mathrm{c}$. The Booster circular accelerator takes protons from the linac and accelerates them up to $8 \mathrm{GeV} / \mathrm{c}$. The Booster then feeds the protons into the Main Injector (MI) circular accelerator which accelerates the protons up to $120 \mathrm{GeV} / \mathrm{c}$ which finally feeds into the Tevatron. While accelerating the protons up to $120 \mathrm{GeV} / \mathrm{c}$ the MI forms the protons into seven batches in the MI. Of these seven, two would go to the Tevatron while the rest would be used 


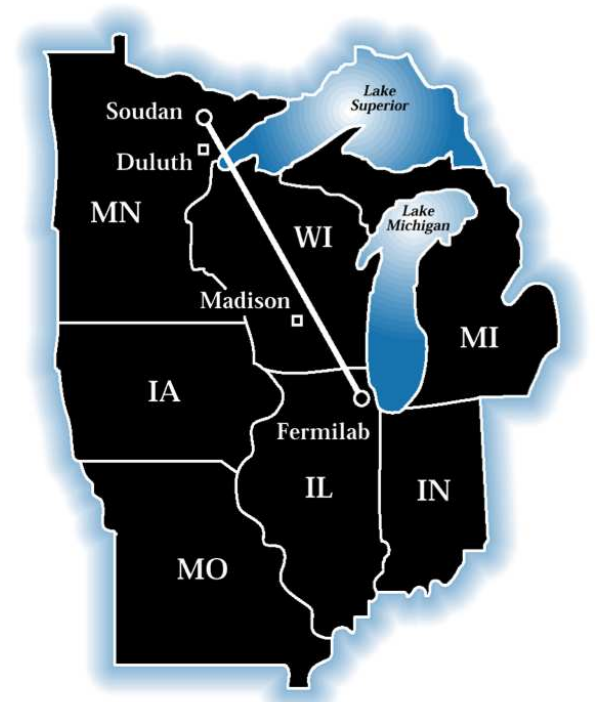

Figure 4.1: From [66]. Geograpical view of the MINOS experimental layout.

for neutrino production at NuMi. The Fermilab accelerator layout is shown in Figure 4.2.

It takes $\sim 1.6 \mathrm{~s}$ for the entire acceleration process to complete at which point the protons in the MI are extracted to either the Tevatron or the NuMI. The beam of protons used for NuMI are bent downward at an angle of $58 \mathrm{mrad}$, which is pointed right at the FD $734 \mathrm{~km}$ away and $2312 \mathrm{ft}$ underground. The protons batches are extracted from the MI in 8.6 $\mu$ s spills which usually contained $\sim 2.1 \times 10^{13}$ protons giving the MI an average beam power of up to $400 \mathrm{~kW}$.

NuMI uses a water-cooled graphite rod as an interaction target for the protons from the MI (Figure 4.3). The target measures $6.4 \times 15 \times 940 \mathrm{~mm}^{3}$, and is segmented into forty-seven fins in the longitudinal direction. The target was designed long and narrow to maximize the longitudinal interactions of the primary proton beam while simultaneously minimizing the reabsorption and re-interactions of the secondary hadron showers. The proton collisions within the carbon target produce a spray of mainly pions and kaons. These secondary particles are focused or defo- 


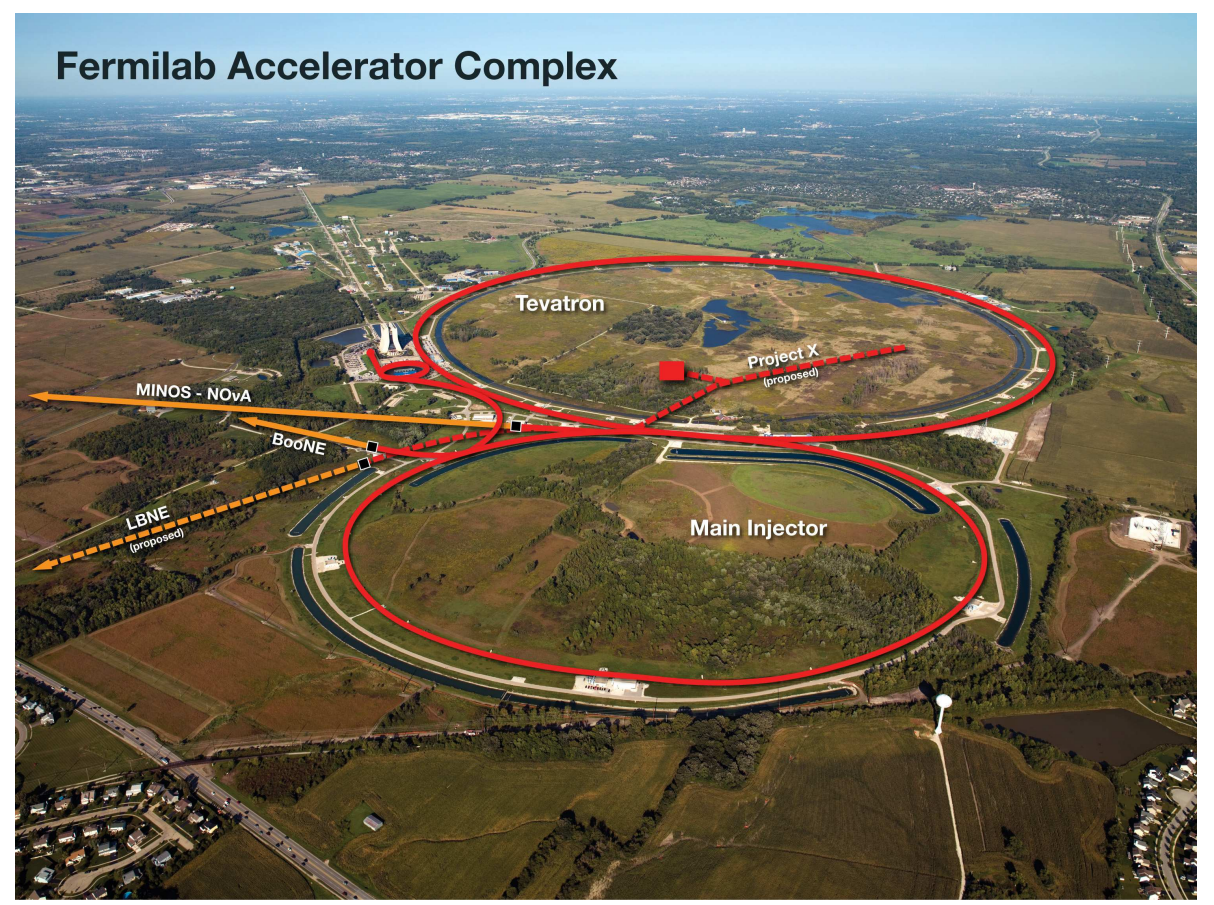

Figure 4.2: From [66]. The layout of the various FNAL proton accelerators.

cused (depending on particle charge) by a pair of parabolic focussing horns. The horns are pulsed with a nominal current of $185 \mathrm{kA}$ which produces a toroidal magnetic field with a maximum strength of $30 \mathrm{kG}$. These horns act as magnetic 'lenses' that selects the secondary hadrons based on hadron momentum and charge sign, for example in neutrino mode the horn would 'focus' $\pi^{+}$while 'defocusing' $\pi^{-}$. The focusing horns are shown in Figure 4.4.

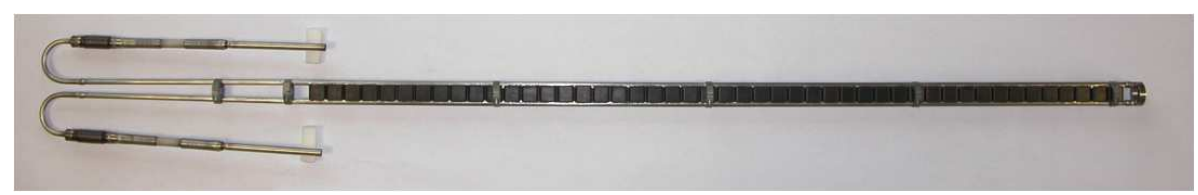

Figure 4.3: From [66]. The NuMI target. 
Once the charged hadrons have been focused (or defocused) by the magnetic horns, the focused particles enter a $675 \mathrm{~m}$ long, evacuated (and later helium filled) decay pipe. Here many of the secondary hadrons (mostly pions and kaon) decay to neutrinos via a process similar to $\pi \rightarrow \mu^{+}+v_{\mu}$. Hadrons that do not decay within the decay pipe are stopped by a $5 \mathrm{~m}$ hadron absorber which consists of a water cooled aluminum core surrounded by steel blocks and a layer of concrete.
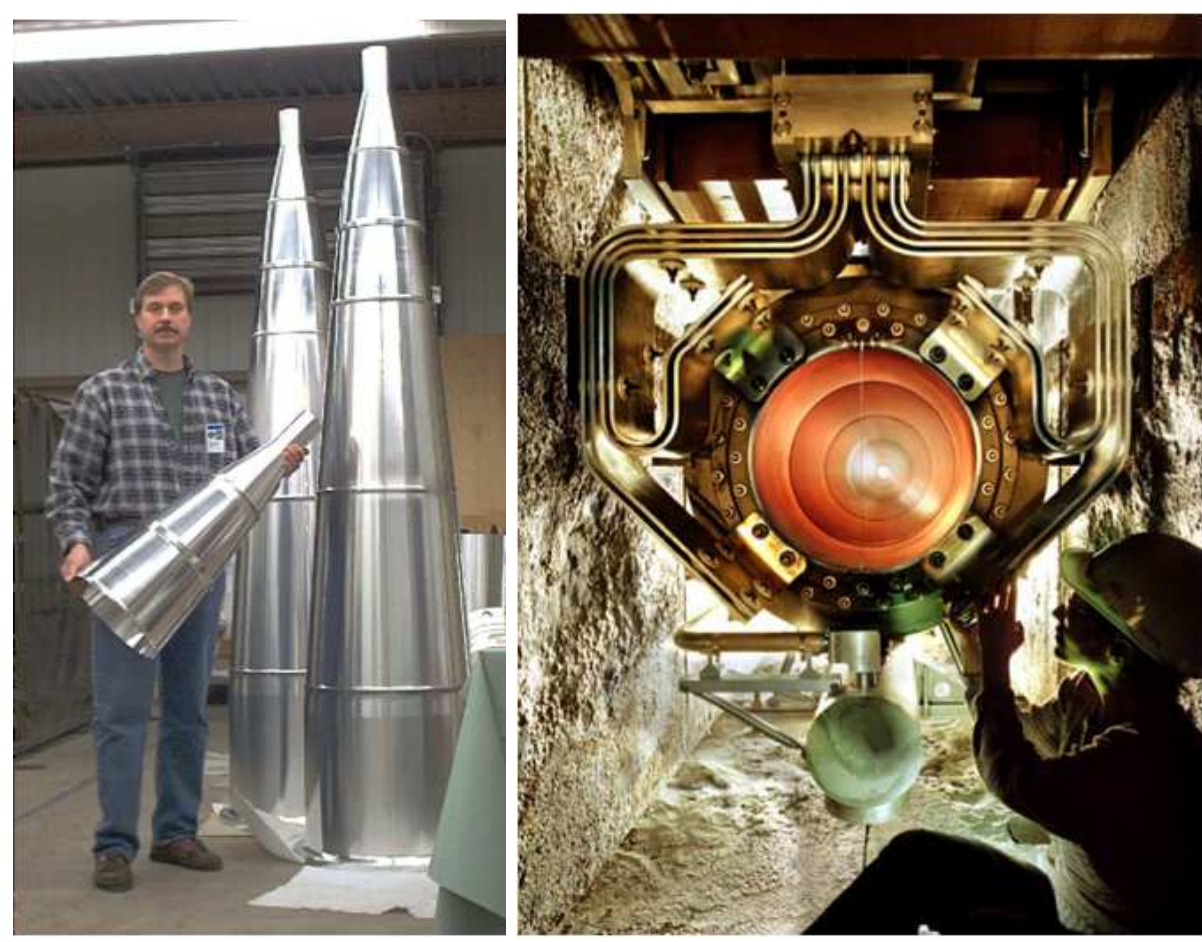

Figure 4.4: From [66]. Left: Photograph of inner conductors of the NuMI parabolic focusing horn. Right: Photograph of the first focusing horn.

After the undecayed hadrons have been stopped by the hadron absorber, the tertiary beam of muons are stopped by the $300 \mathrm{~m}$ of dolomite rock that lies between the NuMI target and the MINOS ND cavern. This leaves a beam of the neutrinos whose path will take them through the MINOS ND and later the MINOS FD. A schematic of the various components of the NuMI beam line is shown 
in Figure 4.5. The NuMI beam is composed of $\sim 97.8 \% v_{\mu}$ with the majority of the contamination coming from $\sim 1.8 \% \overline{\mathrm{v}}_{\mu}$ and the remaining $\sim 0.4 \% \mathrm{v}_{e}$. These estimates come from a MC simulation of the neutrino beamline and hadron production off of the NuMI target.

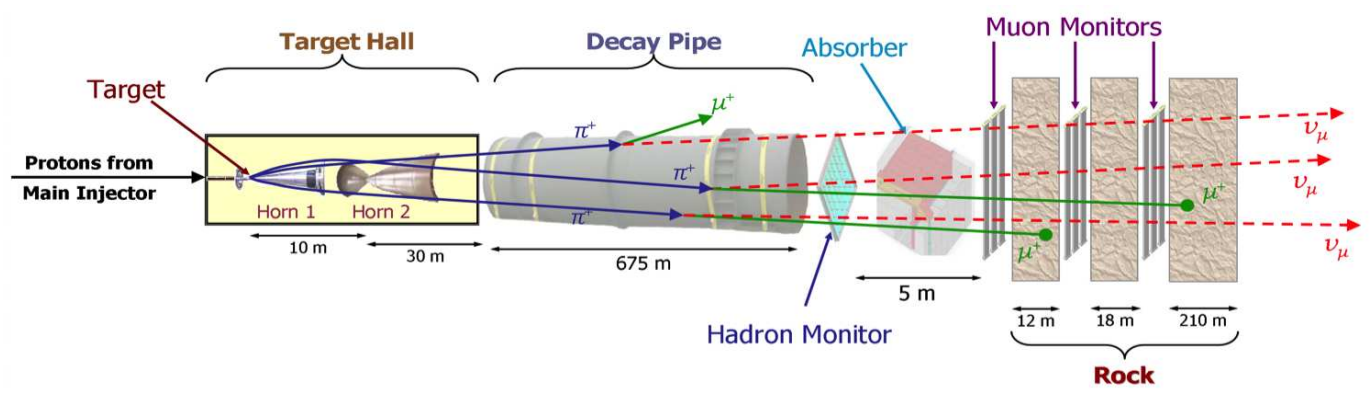

Figure 4.5: From [66]. The various components of the NuMI beamline.

NuMI was designed to be capable of many different experimental modes. The neutrino energy spectrum can be tuned by changing either the current going through the focusing horns or changing the position of the target relative to the first focusing horn. These changes result in the focusing of different momentum hadrons, which in turn leads to different energies of the tertiary neutrino beam. The direction can also be reversed which changes the charge sign of the particles focused and defocused which will produce a beam composed of predominantly $\overline{\mathrm{v}}_{\mu}$.

\subsection{The MINOS Detectors}

Throughout the development of the MINOS experiment three distinct detectors have been used: the Near and Far detectors which measure the energy spectrum of the NuMI neutrino beam, and the smaller Calibration detector (CalDet) which was exposed to a test beam at the CERN proton synchrotron. The results from CalDet are used to characterize the response of the other MINOS detectors to 
electrons, muon, protons, and pions. The MINOS detectors are designed to be as identical as possible given the given the often conflicting demands of expense and experimental sensitivity. By making the detectors functionally identical, the systematic uncertainties that would be associated with the two detector configuration of the oscillation analysis are minimized.

\subsubsection{Common Detector Features}

All of the MINOS detectors are steel-scintillator tracking calorimeters. The Near and Far detectors are magnetized with toroidal fields that have a field strength up to $1.5 \mathrm{~T}$. The magnetic fields allow for the measurement of both the muon momentum by path curvature and muon/anti-muon identification.

These detectors consist of a number of steel-scintillator planes. Each plane consists of $2.54 \mathrm{~cm}(1 \mathrm{in})$ thick steel plate that is attached to a $1 \mathrm{~cm}$ thick layer of segmented scintillator strips. The detectors are composed of large numbers of these planes, hung perpendicularly to the neutrino beam, with a $2.4 \mathrm{~cm}$ air gap between each plane. The iron in the steel planes provide $95 \%$ of the interaction nucleons with the remaining interaction nucleons provided by carbon and other trace elements. While the interaction targets are provided by the steel planes, the scintillator strips provide the active detector element.

The layers of scintillator consist of strips $1.0 \mathrm{~cm}$ thick by $4.1 \mathrm{~cm}$ wide of varying length (up to $8 \mathrm{~m}$ long). They are primarily composed of polystyrene that has been doped with flours 2,5-diphenyloxazole (PPO) at 1\% and 1,4-bis-2-(5phenyloxazolyl)-benzene (POPOP) at $0.03 \%$. The strips are arranged side by side and encased in aluminum, which forms the scintillator strips into light tight modules which are mounted onto the steel plates. The strips are oriented in such a way that they are orthogonal to adjacent planes. This orientation allows for three dimensional reconstruction of the neutrino interaction products within the detectors.

As particles pass through the scintillator strips they occasionally excite electrons in the flours, when these electrons de-excite they emit light, the emitted 
light is collected by $1.2 \mathrm{~mm}$ diameter wavelength shifting (WLS) optical fibers. The optical fibers are glued into a $2 \mathrm{~mm}$ groove that runs along the center of the scintillator strip. The WLS fiber change the average wavelength of the emitted light from blue region $(460 \mathrm{~nm})$ to the green region $(530 \mathrm{~nm})$. The strips are coated with a $\mathrm{TiO}_{2}$ doped paint, which helps to reflect and trap the scintillation light, which maximizes the likelihood that the light will be collected by the WLS fibers. The WLS fiber is routed from the individual strips and collected into a manifold which guides the fibers into an optical connection. Figure 4.6 shows an individual scintillator strip along with strips that have collected into strip module.

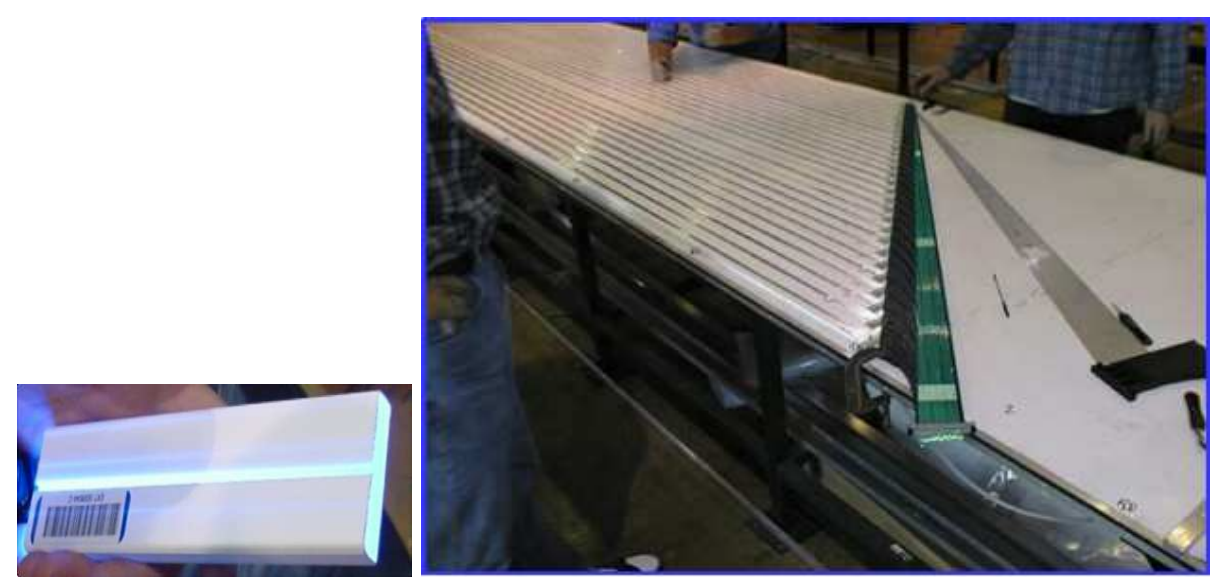

Figure 4.6: From [66]. Left: Individual scintillator strip. Right: Strips that have collected into a detector module.

Once photons reach the optical connector, they are routed via clear optical fibers to multi anode photomultiplier tubes (PMTs). Clear optical fibers are used because they have a much longer attenuation length. The PMTs are used to turn the optical signal from the scintillator into an electronic signal that can be processed by the data acquisition (DAQ) system. A schematic of this system is shown in Figure 4.7. 

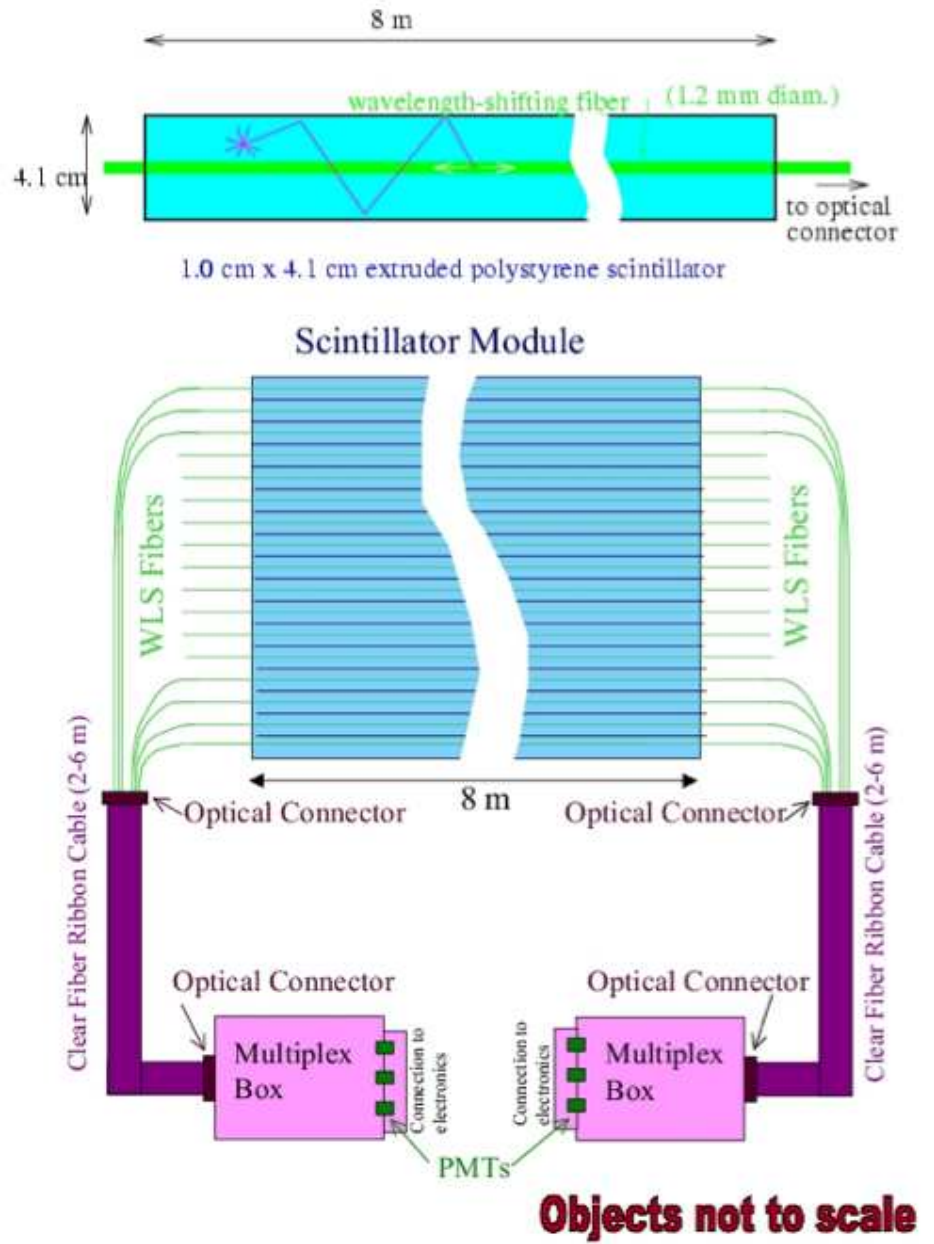

Figure 4.7: From [66]. Schematic of a scintillator plane readout system.

\subsubsection{The Calibration Detector}

The Calibration detector weighed 12 tons and was constructed to measure the response and topology of the Near and Far detectors to hadronic and leptonic particles passing through the detector elements. CalDet was active from 20012003 and exposed to beams composed of $p^{+}, e^{ \pm}, \mu^{ \pm}$, and $\pi^{ \pm}$in the few-GeV 
region at the CERN proton synchrotron accelerator. CalDet consisted of sixty $1 \mathrm{~m}$ square planes, each plane is composed of twenty four scintillator strips. CalDet was configured so that it could be operated with electronics and readouts that either simulated the Near or Far detector systems. CalDet was also equipped with a time-of-flight system, which acted as a trigger and discriminated between $e, \pi$, and $p$, and a Cherenkov counters that were used to tag electrons in the detector. A photograph of the Calibration detector is shown in Figure 4.8.

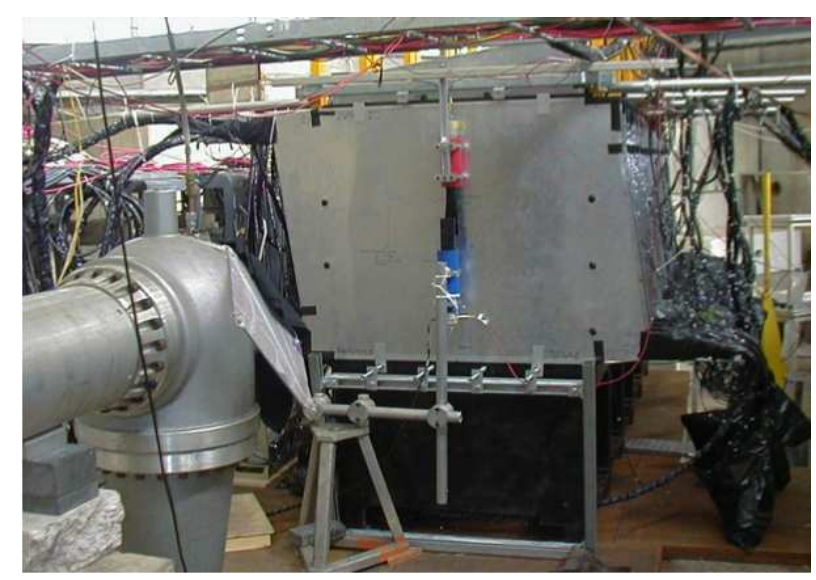

Figure 4.8: From [66]. Photograph of the MINOS Calibration detector.

\subsubsection{The Far Detector}

The MINOS Far detector, at $5.4 \mathrm{kT}$ is the largest of the MINOS detectors. The Far detector lies in the Soudan Underground Laboratory in Soudan Minnesota $2312 \mathrm{ft}$ (705 m) underground and $735 \mathrm{~km}$ away from the NuMI target. The Far detector is composed of a total of 484 planes formed into an octagonal cylinder that measures $8 \mathrm{~m}$ across. Due to limitations on the length of the coil that supplies the magnetic field the to Far detector, the Far detector is divided into two 'supermodules', separated by an air gap of $1.2 \mathrm{~m}$. The individual supermodules are composed of 249 planes and 237 planes, and each are independently magnetized. Each of the coils 
are supplied by a current of $\sim 15 \mathrm{kA}$ which produces an average magnetic field of $\sim$ '1.27 T. A photograph of the completed Far detector is shown in Figure 4.9.

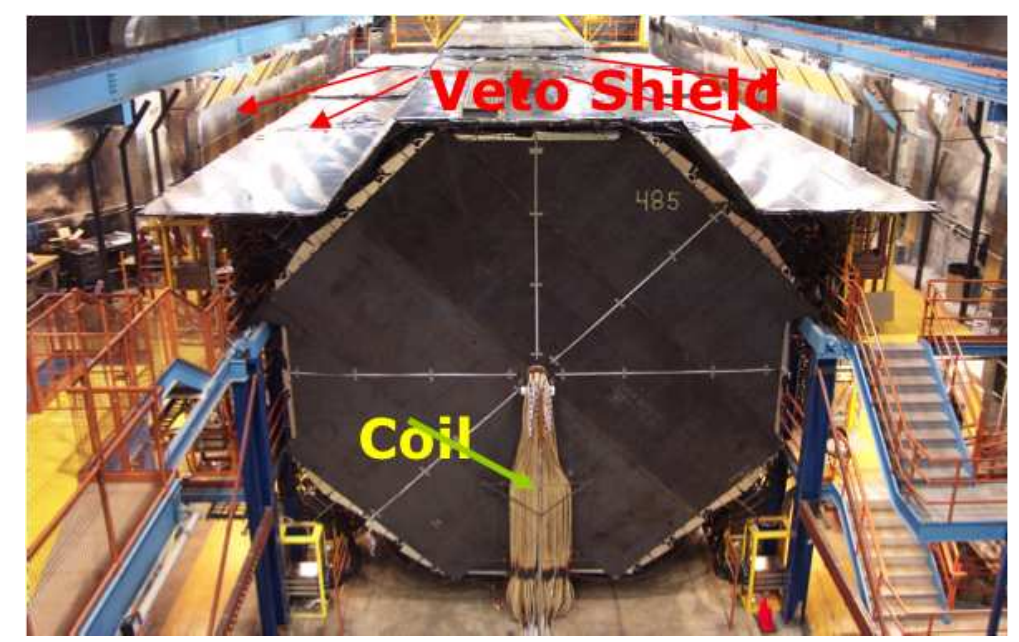

Figure 4.9: From [66]. Photograph of the completed Far detector.

Each plane is constructed with 192 scintillator strips, which are laid out side by side. The strips are oriented at $45^{\circ}$ from the horizontal and read out from both ends by Hamamatsu M16 PMTs. Eight different strip ends are read out by a single PMT pixel. This 'multiplexing' technique was done to minimize the expense of PMTs due to the large number of strips ends that need to be read out (185,856 strip ends). However multiplexing introduces an ambiguity when trying to perform three-dimensional reconstruction, to resolve this ambiguity the strip-to pixel pattern is different for each side of the strip readout.

The FD front end electronics use a multi-channel application specific integrated circuit (ASIC), these ASIC's are also known as 'VA chips'. One VA chip is used with each M16 PMT. The FD was designed to operate continuously, thus each PMT was configured in such a way that they can trigger independently. The function of the VA chip is to digitize the signals from the PMTs, which they do with a precision of $2 \mathrm{fC}$. The PMTs are triggered when the summed signal in every pixel in a single PMT exceeds a threshold of $\sim 0.25$ photoelectrons. Finally these 
front end electronic boards time stamp the signal with a precision of $1.56 \mathrm{~ns}$.

The signal collected from the front end boards are pedestal suppressed, then passed to a computer farm, the computer farm then applies higher level triggering conditions which help determine whether the data should be saved. The primary triggering condition is the 'spill trigger', which determines whether the FD readout is coincident with a beam spill from the MI. GPS clocks are used to the synchronize the Near and Far detectors. When a beam spill occurs at the MI, a time stamped signal is sent to the FD through an internet connection. The FD will record all of the hit information in a time window $80 \mu$ s wide and centered on the time of the MI beam spill. This 'spill window' can be extended to ensure that there is an activity free period of at least $156 \mathrm{~ns}$ so that a candidate neutrino event wont be split into two separate spill windows.

\subsubsection{The Near Detector}

The Near detector is the smaller of the two primary MINOS detectors with a mass of $\sim 980$ tons, it is located $\sim 320 \mathrm{ft}$ ( $\sim 98 \mathrm{~m}$ ) underground on site at FNAL $\sim 1 \mathrm{~km}$ from the NuMI target. Because the neutrino beam has diverged very little in the $\sim 1 \mathrm{~km}$ between the target and the ND, and NuMI has an inherently high neutrino rate, the ND was designed to be able to contend with this high interaction rate. The ND is composed of 282 planes arranged in a 'squashed' octagonal shape, where each plane is $6.2 \mathrm{~m}$ wide and $3.8 \mathrm{~m}$ high. There is a $30 \times 30 \mathrm{~cm}^{2}$ hole offset $0.56 \mathrm{~m}$ from the horizontal center of the ND through which the coil that supplies the magnetic field passes through. The center of the neutrino beam spot lies $0.93 \mathrm{~m}$ from the horizontal center of the plane, in the opposite direction of the coil. The magnetic field coil carries a current of 40kA which creates a magnetic field of $1.17 \mathrm{~T}$ in the vicinity of the beam center. Figure 4.10 shows a photograph of the Near detector.

The ND is divided into two different components: the first 120 upstream planes compose the calorimeter region, while the remaining downstream planes compose the spectrometer region. The calorimeter region is designed to maxi- 


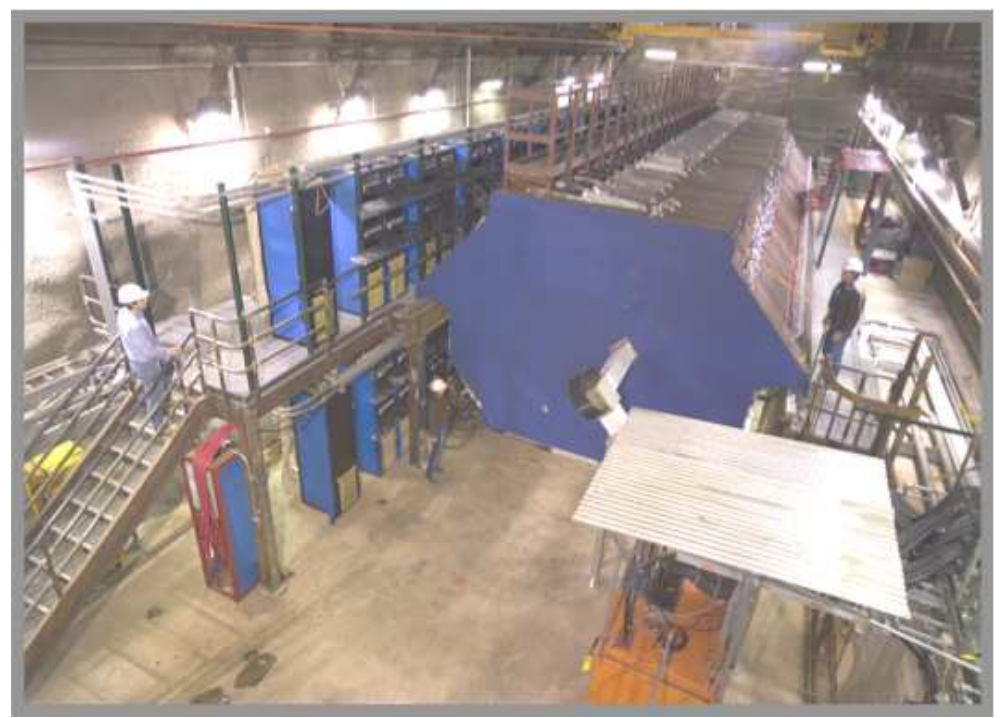

Figure 4.10: From [66]. Photograph of the Near detector.

mize the precision of interaction vertex and vertex activity measurements, while the spectrometer is designed to measure the momentum of the muons that are produced via the neutrino interactions in the calorimeter region.

The calorimeter region of the ND is the first 120 planes of the detector. While every plane of the calorimeter is instrumented with scintillator strips, only a fifth of the planes are fully instrumented. The remaining four-fifths of the planes are only partially instrumented. The scintillator strips in the partially instrumented region are centered around the beam center. These scintillator strips are sufficient for measuring the development of hadronic showers near the interaction vertex.

The spectrometer region is the final 142 downstream planes of the ND. The spectrometer continues the every fifth instrumented plane sequence of the calorimeter, only the partially instrumented planes are excluded. The spectrometer region is used to measure the momentum of muons produced within the calorimeter region. This momentum measurement can be done using two different means: if the muon stops in the detector the momentum can be measured via range, or if the muon exits out of the detector the magnetic field can be used to measure the 
momentum from curvature of the track. A schematic illustrating the differences between a partially instrumented and a fully instrumented plane is shown in Figure 4.11 .
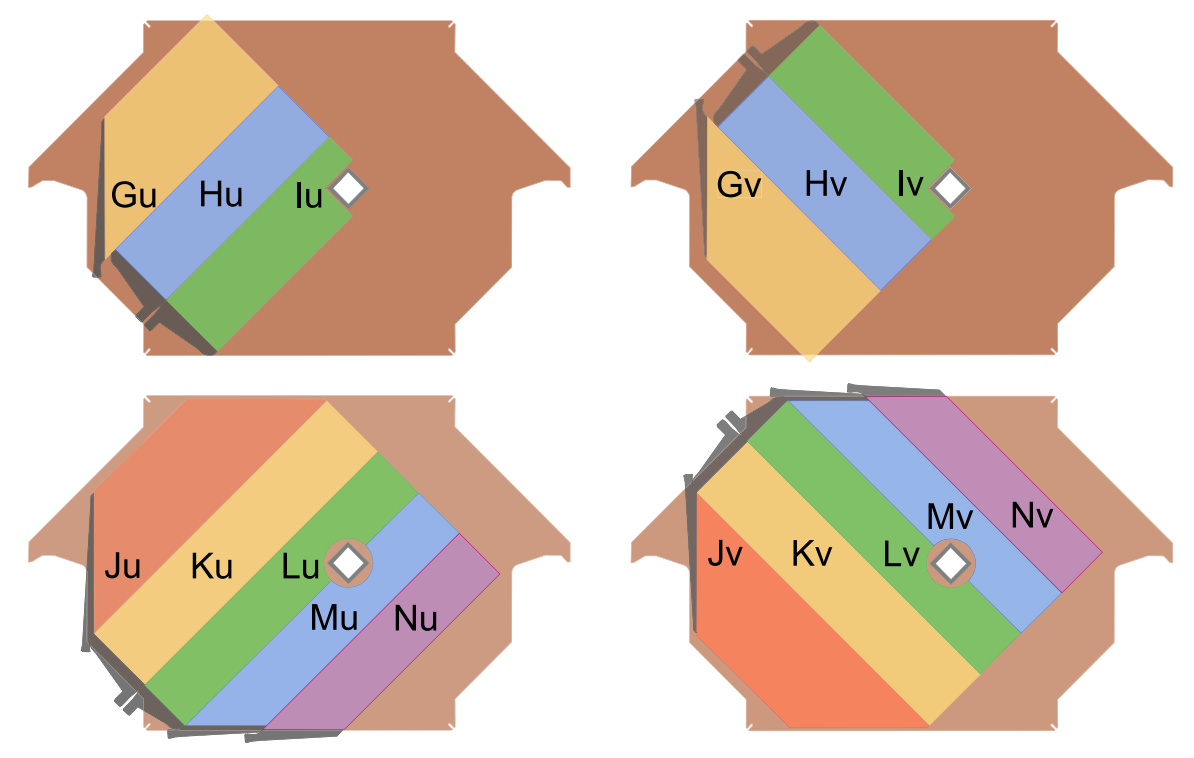

Figure 4.11: From [65]. Strip configuration of the MINOS ND, divided into partially instrumented (above) and fully instrument (below)planes, and also U-view (left) and V-view (right) planes.

The ND strips are readout from only one end (unlike the FD). This is because the scintillator strips for the ND are shorter than the scintillator strips used in the FD. To compensate for the single read out end and also to maximize the light collection, the end opposite from the read out end is coated with a reflective material. These strips are read out using Hamamatsu M64 multi-anode PMTs, each partially instrumented plane requires a single PMT for readout, while a fully instrumented planes requires one and a half PMTs for readout. The amount of instrumentation is reduced in the spectrometer by summing the signal from sets of four pixels into a single electronics channel. The adjacent pixels are not connected to adjacent strips, thus the four-fold ambiguity can be resolved through the tracking of events 
in the calorimeter (where there is no ambiguity) into the spectrometer allowing for discrimination between the four possibilities. Thus it is not possible to resolve this ambiguity for events whose vertex doesn't lie within the calorimeter.

On average there are sixteen neutrino interactions in the ND during each beam spill (when in the L010z185i beam configuration). Thus the ND requires special high speed and deadtime less front end electronics. This electronic system is based on the QIE chips that were used in the CDF and KTEV experiments and developed at FNAL. The primary trigger is the spill trigger, when triggered for a $13 \mu$ s window the output of every PMT pixel is readout starting $1.5 \mu$ s before the beamspill. 


\section{Chapter 5}

\section{MINOS Data and Monte Carlo}

\subsection{MINOS Data}

This analysis will use data collected using just the Near detector. The data was collected during the first MINOS data taking run, from May 20, 2005 through February 2, 2006. This constituted $\sim 1.27 \times 10^{26}$ protons on target (POT) at NuMI. The data as originally recorded by the MINOS ND consists of PMT pixel pulse height as a function of time with nanosecond precision. This information about single detector strips needs to be calibrated for the individual strip along with a calibration for the location along the strip. Then the reconstruction software associates the information about individual hits into higher level reconstructed quantities such as tracks and showers, which are grouped into events (tracks and showers that are associated by space and time in the detector) ideally these events represent a single neutrino interaction within the detector.

\subsubsection{Reconstruction}

The MINOS reconstruction software uses the raw data of the energy deposited within a strip and the strip location within the detector to attempt to identify the path of individual higher energy particles (tracks) or cascades of lower energy par- 
ticles (showers). In the ND there are up to sixteen neutrino interactions per beam spill. Every neutrino interaction will produce several secondary particles. Thus there will be as few as 20 and possibly many more potentially visible particles produced in the ND in a single beam spill. The first reconstruction step in the ND is to group together hits that are near each other (both spatially and temporarily). These slices of the beam spill trigger are assumed to be due to a single neutrino interaction.

\section{Tracks}

When a massive charged particle relativistically passes through matter, the particle looses energy through the ionization of the surrounding material and through the excitation of nearby nuclei. The rate of energy loss is given by [67], which contains updated tables of muon energy loss in matter. Included in [67] are updates to the Bethe-Bloch theory of ionization energy loss [68]. The Bethe-Bloch equation describes the energy loss (due to ionization) of particles as they pass through matter, the full Bethe-Bloch equation is:

$$
-\frac{d E}{d x}=K z^{2} \frac{Z}{A} \frac{1}{\beta^{2}}\left[\frac{1}{2} \ln \left(\frac{2 m_{e} c^{2} \beta^{2} \gamma^{2} T_{\max }}{I^{2}}\right)-\beta^{2}-\frac{\delta(\beta \gamma)}{2}\right]
$$

where

$$
T_{\max }=\frac{2 m_{e} c^{2} \beta^{2} \gamma^{2}}{1+2 \gamma m_{e} / M+\left(m_{e} / M\right)^{2}}
$$

is the maximum kinetic energy that can be transferred to a free electron in a single collision. $z$ is the charge of the incident particle relative to the electrons charge, $I$ is the average excitation energy of the medium, $Z$ is the atomic mass and $A$ is the atomic number or the medium. $m_{e}$ is the mass of the electron, $M$ is the mass of the incident particle. $K$ is an amalgamation of several constants: $K / A=$ $4 \pi N_{A} r_{e}^{2} m_{e} c^{2} \approx 0.307 \mathrm{MeVg}^{-} 1 \mathrm{~cm}^{2}$ for $A=1$, where $r_{e}$ is the charge radius of the electron and $N_{A}$ is Avogadro's number.

In Equation 5.1, the $\delta(\beta \gamma)$ term is a correction that accounts for ionization 
energy lossed due to the effect of the medium density, this term can be neglected for $\beta \gamma \approx 100$. There are other possible radiative processes when charged particles pass through matter, such as bremsstrahlung and $e^{+} e^{-}$pair production, however these effects can be neglected for muons with energy less than $\sim 400 \mathrm{GeV}$ [68].

The minimum of the Bethe-Bloch equation (Equation 5.1) as a function of $\beta \gamma$ occurs between $2<\beta \gamma<4$, while the Bethe-Bloch equation plateaus at higher energies, where $\frac{d E}{d x}$ is slightly larger than $\left.\frac{d E}{d x}\right|_{\text {min }}$. Particles with $\beta \gamma$ near the minimum energy loss are called Minimum Ionizing Particles (MIP). Muons produced in the ND from CC interactions are usually relativistic, and thus are minimum-ionizing. After a particle has lost enough energy to no longer be minimum-ionizing the particle will lose increasingly more energy per distance traveled. The stopping power of muons as a function of muon momentum for both data and $\mathrm{MC}$ along with the Bethe-Bloch equation is shown in Figure 5.1.

Muons in the ND are produced with energy $\gtrsim 1 \mathrm{GeV}$, and are thus relativistic and minimum-ionizing. The kinetic energy of the muons produced within the ND can be estimated to first order by simply counting the number of planes that the muon passes through before the muon stops. This approximation improves as the muon gets closer to normal incidence, where the approximation becomes exact. This is unlikely to happen because the muon will usually carry some transverse momentum from the neutrino $\mathrm{CC}$ interaction, and the curvature due to the magnetic field. MINOS has good energy resolution from $\frac{d E}{d x}$ in the steel planes, with $\sigma_{E_{\mu}}^{\text {stop }}=2 \%$. The uncertainty in the energy of the stopping muon track is due to the uncertainty in the total path length of the muon and the uncertainty in the thickness of the individual planes.

While it is possible to measure the muon energy from the muon's track length, this requires that muon stop within the ND, it is also possible that the muon exits the instrumented region of the detector before stopping. This makes an estimate of the muon energy from the Bethe-Bloch equation impossible. When a muon exits the the instrumented region of the ND before stopping it is still possible to estimate the muon momentum from the curvature of the track. Estimating the 


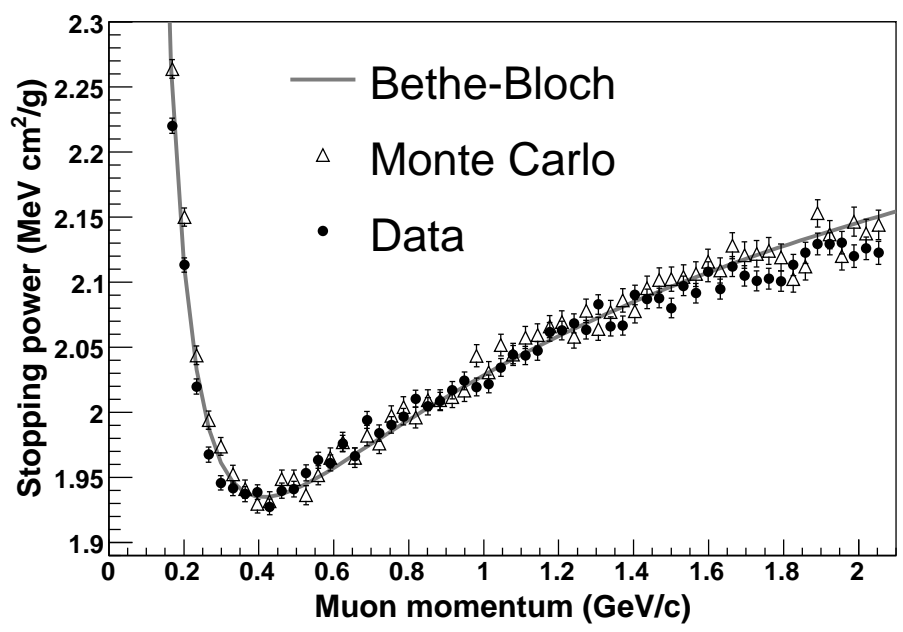

Figure 5.1: From [65]. Stopping power for muons. The gray line shows the Bethe-Bloch calculation of the stopping power for muons in polystyrene scintillator. The solid circles and open triangles show the response of stopping muons in the far detector data and GEANT3 Monte Carlo simulations respectively. Both data and Monte Carlo points have been normalized to the Bethe-Bloch calculation to give the expected stopping power at the minimum ionizing point.

muon momentum from track curvature is done through the use of a Kalman filter algorithm [69][70].

The Kalman filter algorithm is a iterative algorithm for calculating the path of an object based on sparse (and potentially incomplete) data. The advantage of using the Kalman filter is that the filter can apply known physics to assist the algorithm as the algorithm attempts to make predictions. The Kalman filter predicts the value of five different parameters that describe the muons path through the detector medium and the applied magnetic field, these parameters are $u, v, \frac{d u}{d z}, \frac{d v}{d z}$, and $q / p$, where $u, v$ are the transverse position of the strips in the detector coordinate system, $z$ is the longitudinal position of the strips, $q$ is the charge expressed relative to the electron's charge, and $p$ is the momentum of the muon. The first four variables are known from point to point and serve as input at each iteration 
step, while the last variable $(q / p)$ is the final output of the Kalman filter. The muon is tracked by the Kalman filter through the entire length of the track, it takes both energy loss due to ionization and the bending of the muon's path due to the magnetic field into account when making predictions for the next iteration.

Another output of the Kalman filter is an error matrix that expresses the accuracy of the filter's predictions at each iteration step. Information from the error matrix can be used to calculate an estimate of the uncertainty in the quantity $q / p$, $\sigma_{q / p}$. Multiple scattering of the muon is the most important contribution to the estimation of $\sigma_{q / p}$. Multiple scattering is the scattering of the muon off of many different nuclei, which causes small perturbations in the muon's position (and their derivatives) within an individual strip. These small perturbations cause the measurement of the muon's momentum from curvature to be less precise than the momentum from range, the uncertainty in the muon momentum from curvature is $5 \%$.

\section{Showers}

No attempt is made by the standard MINOS reconstruction to reconstruct individual particles produced as a result of neutrino induced hadronic showers. Instead, calorimetric information about the showers are used to estimate the total energy of the shower.

When a neutrino interacts with a nucleus, the neutrino will transfer some fraction of it's initial momentum to the scattering target. Often this transferred momentum is enough to produce a shower large enough to be visible in the detector. Because the secondary particles produced during a hadronic shower are hadrons, the secondary particles will lose energy by interacting via the strong force, this will produce more low-energy hadrons, and though many of the particles produced during a hadron shower will also lose energy through the Bethe-Bloch process, the dominant processes is through strong interactions. As hadronic showers develop many different strong processes along with nuclear processes effect the development of the shower. Some of these processes such as pion absorption and 
neutron production are very difficult to see within the MINOS detectors. These effects cause the resolution on the shower energy to be large (relative to the muon track energy). This energy resolution along with the absolute energy measurement is very dependent on the energy calibration of the detectors, which is discussed in the next section (Section 5.1.2).

\subsubsection{Calibration}

Calibration of the MINOS detectors is done by combining information from the CalDet detector, cosmic-ray muons, test-bench measurements of the scintillator systems, and a LED-based light-injection (LI) system. The purpose of the calibration procedure is to receive as input the raw detector output $Q_{\text {raw }}(d, s, t, x)$ and convert it into a fully corrected signal $Q_{c o r}$ where $d$ is the detector used, $s$ is the strip the signal comes from, $t$ the time the signal was recorded, and $x$ is the position along the strip from which the signal originated. This correction is done through the use of calibration-constants which are scale factors on the raw signal $Q_{\text {raw }}$ :

$$
Q_{\text {cor }}=Q_{\text {raw }} \times D(d, t) \times L\left(d, s, Q_{\text {raw }}\right) \times S(d, s) \times A(d, s, x) \times M(d)
$$

the constants $D, L, S, A, M$ correspond to the following corrections:

- Drift Correction $(D)$ : a correction that removes the changes to the detector response as function of time. Detector components, such as PMTs, detector electronics, and the scintillator can change their response simply due to the passage of time. This correction is an attempt to account for those changes. This correction was originally performed use the LI data, to measure the individual changes of the strips, however it was discovered that the time drift of the individual strips was consistent enough for the drift calibration to be performed from the mean signal per plane that comes from cosmic ray muons that completely pass through the detector. Any remaining differences between strip outputs can be corrected away by the strip-to-strip cor- 
rection. Figure 5.2 shows the $\%$ change in the detector response over from the start of data taking through the end of year 2007. There was $< \pm 1 \%$ change in detector response for the data taking period this analysis.
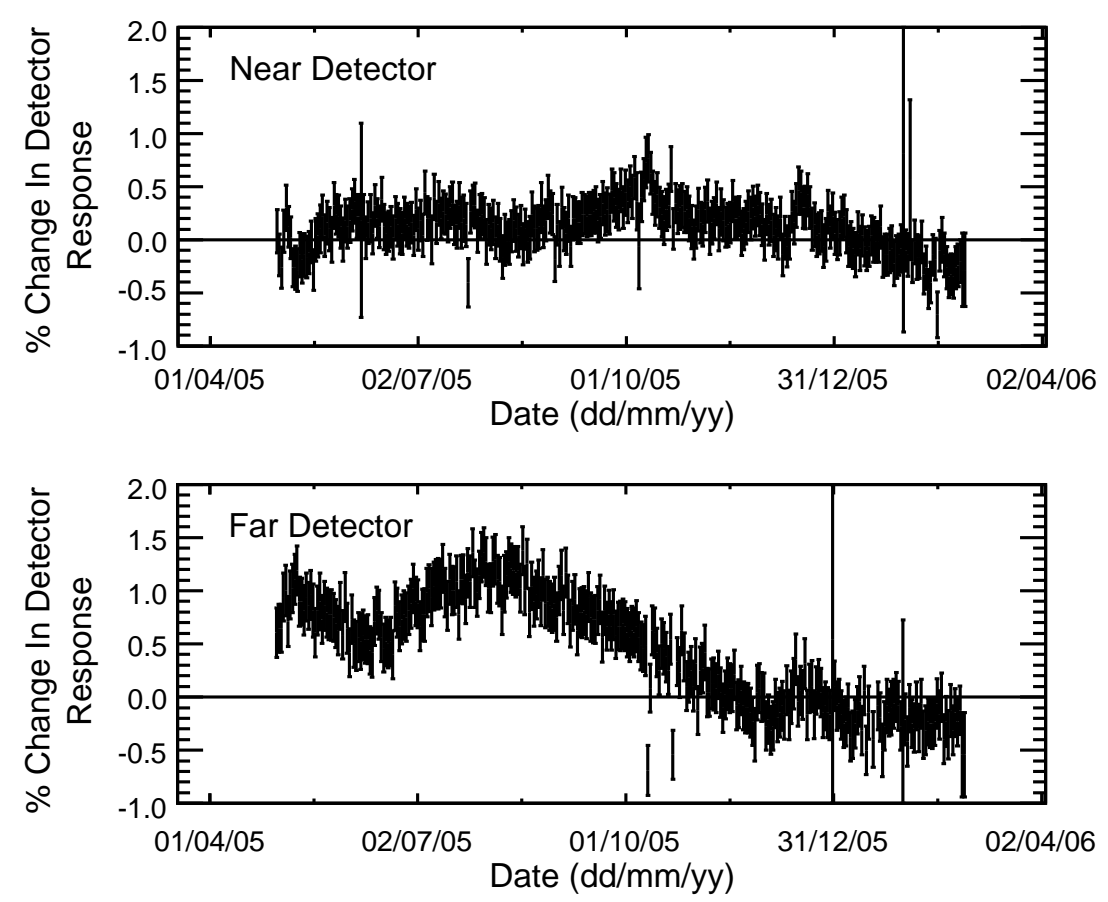

Figure 5.2: From [71]. Variations in the median signal per plane deposited by through-going cosmic-ray muons observed during the data-taking period covered by this paper. The time dependence is largely due to variations in the environmental conditions in the Near and Far Detector halls and aging of the scintillator. The zero point on the ordinate is arbitrary.

- Linearity Correction $(L)$ : a correction that accounts for the non-linearities in the response of the PMTs and readout electronics as a function of the deposited signal, or pulse height (PH). The LI system produces 'gain curves' for each strip, these gain curves describe the response a function of $\mathrm{PH}$. 
These linearity corrections are measured monthly. The deviation from linearity for both the Near and Far detectors is shown in Figure 5.3.
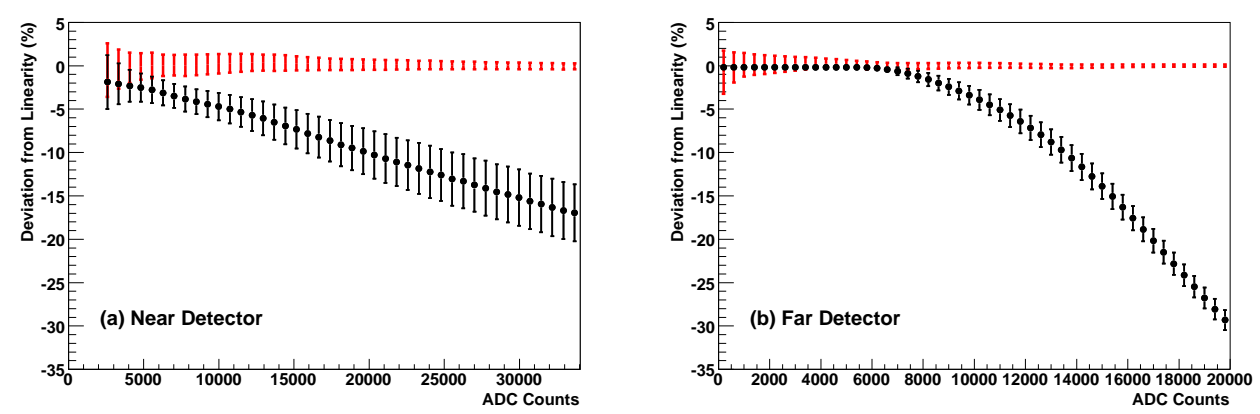

Figure 5.3: From [65]. Nonlinearity calibration of near and far detector instrumentation. The intrinsic nonlinearity of PMT response (circles) and its residual after calibration (error bars only) are shown for the near (a) and far (b) detectors. The error bars depict the rms spread of channels in each detector. For scale, a minimum ionizing particle normal to the plane will generate roughly 500-600 ADCs of charge each detector. A single photoelectron is roughly 75 ADCs (Far) or 100 ADCs (Near).

- Strip-to-Strip Correction $(L)$ : a correction that accounts for the differences between the individual strips. This correction is done by looking at the through going cosmic-ray muons. By accounting for the variations in the light-output between different strips the detector response is made more uniform, this correction uses the attenuation correction on an event-by-event basis to account for the muon path length through the detector, while also taking into account the expected inefficiencies that arise due to the relatively low light levels (the muon may not leave any signal) of the muon tracks. The strip-to-strip calibration constants are measured every month in the ND. The relative response of the strip ends in the ND is shown in Figure 5.4.

- Attenuation Correction $(A)$ : accounts for the attenuation of the signal from each strip. This correction is depends on the position along the length 


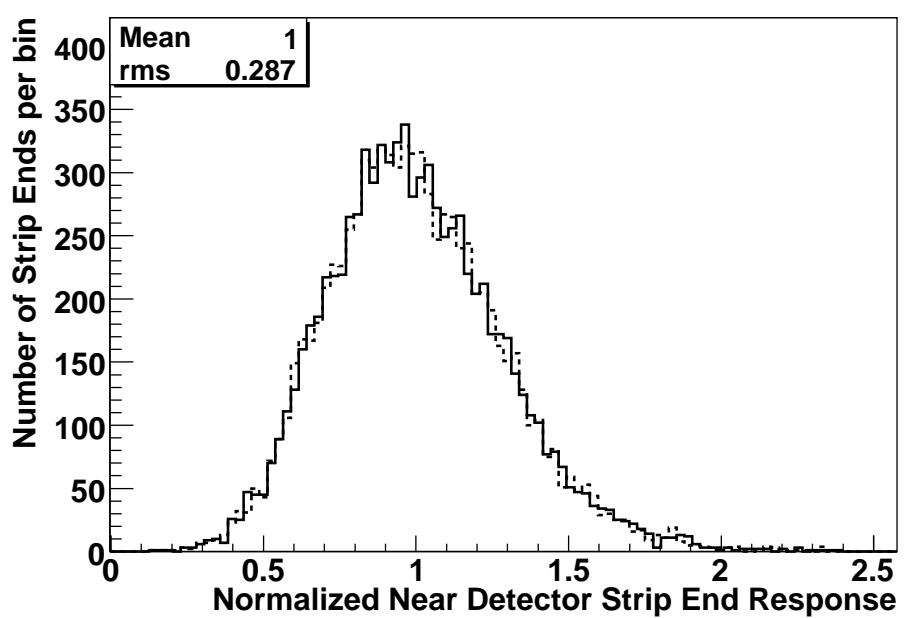

Figure 5.4: From [65]. Mean value of the strip-end responses normalized to the detector average. The mean response of the strip ends varies by approximately 29\%. The solid and dashed lines are the calculated responses of two separate data sets from June 2005. The statistical variation in the individual calibration constants from these two data sets is on the order of $2.1 \%$.

of the strip. The data necessary to calculate these calibration constants was measured in test bench setup prior to installation of the detectors. This test bench setup used a radioactive source to measure the signal output of each scintillator module at several different locations along the length of the modules. These results were fit to a double exponential:

$$
A(x)=A_{1} \exp \left(-x / L_{1}\right)+A_{2} \exp \left(-x / L_{2}\right)
$$

where $x$ is the length along the strip and $L_{1}, L_{2}$ are the attenuation lengths of the WLS fiber that runs along the strip and the clear optical fiber that carries the signal from the optical connectors to the PMTs. Figure 5.5 shows an example fit to the double exponential which is compared to the cosmic ray muon data curve from one module. 


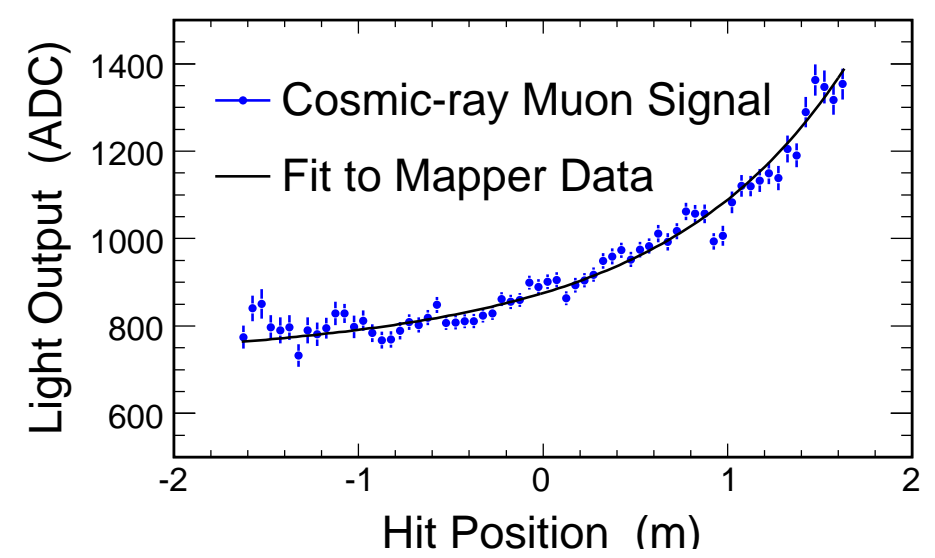

Figure 5.5: From [65]. Comparison of cosmic ray muon data (points) with module mapper fitting results (solid curve) for a typical strip in the near detector.

- Absolute Energy Scale $(M)$ : is a final scale factor that sets the absolute energy scale of the signal that is recored from the individual strips. The calibration constant is calculated from the end region of the cosmic ray muons that stop in the detectors [72]. Stopping the ending parts of cosmic ray muons are used because this is the region of muon energy loss that is best understood. The average response for each strip is calculated, then the mean value from all the strips defines a muon equivalent unit (MEU).

The final conversion factor converts the energy deposited in the detector in MEU into a standard expression of energy the $\mathrm{GeV}$. This needs to be done separately for muon tracks, and hadronic and electromagnetic showers. The MEU to GeV conversion factor for muons, comparisons of the corrected data at the ND and FD is made to the $\mathrm{MC}$ simulation, along with the information on muon stopping power from [67], this gives a MEU to GeV conversion factor of $2.00 \pm 0.02 \mathrm{MeV} / \mathrm{MEU}$.

The results from the CalDet were used to extract a MEU to $\mathrm{GeV}$ conversion factor for electromagnetic and hadronic showers, the CalDet data was compared 
to the hadronic MC simulations. The combination GEANT/GCALOR [73] was found to best describe the CalDet data (shown in Figure 5.6) which agrees with the data at the $1-5 \%$ level. The MEU to $\mathrm{GeV}$ conversion factor was determined to be energy and shower type dependent (hadronic or electromagnetic).

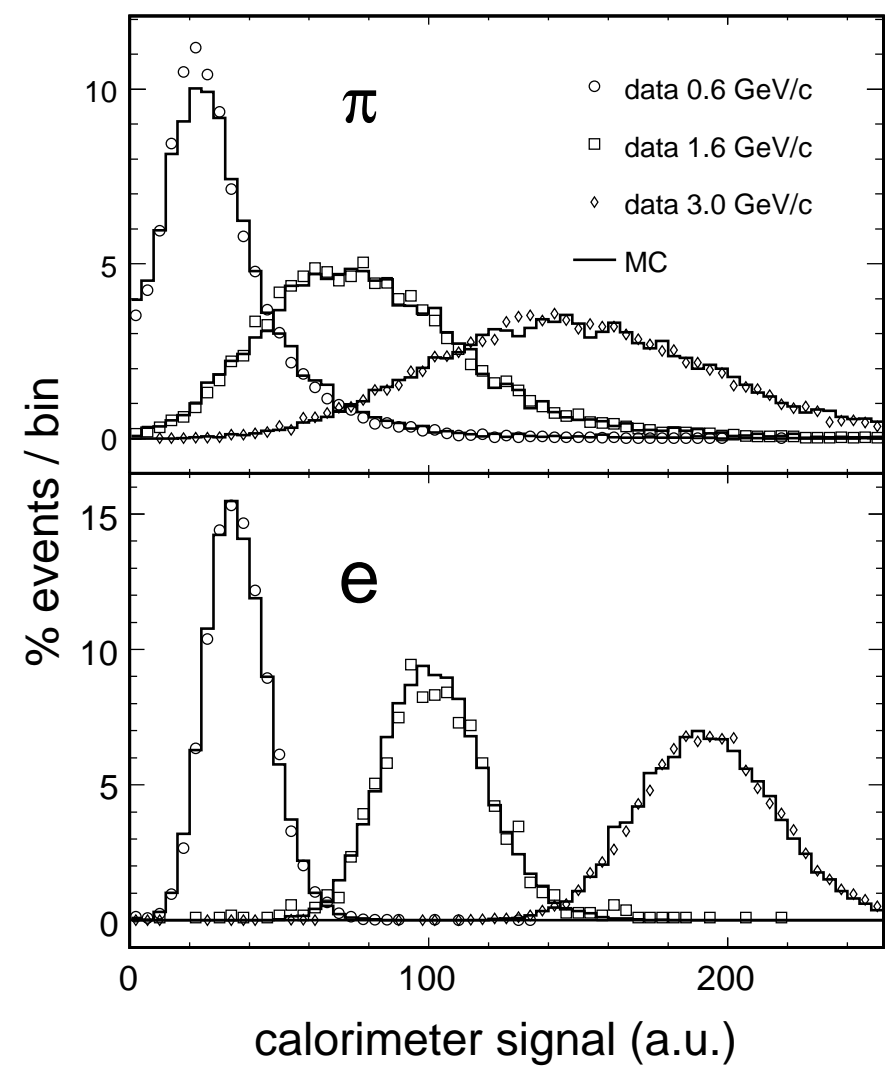

Figure 5.6: From [65]. MINOS calorimetric response to pions and electrons at three momenta. The calorimeter-signal scale is in arbitrary units. The data (open symbols), obtained from the calibration detector exposure to CERN test beams, are compared to distributions from Monte Carlo simulations. 


\subsection{MINOS MC}

MINOS uses a Monte Carlo simulation (MC) to model: the production of hadrons produced by the $120 \mathrm{GeV}$ protons colliding with the NuNI target, the propagation of the hadrons off of the NuMI target through the beam system, the production of the tertiary neutrinos in the NuMI beam, the neutrino interactions within the Near and Far detectors, and the propagation of the secondary particles from the neutrino interactions through the Near and Far detectors.

The FLUKA0 5 MC simulates the hadron production off of and through the NuMI target. The rest of the NuMI beamline is simulated using the GEANT - 3 [74] based software package GNUMI. Every hadronic decay that produce a neutrino is saved to be used later for the neutrino interaction simulation.

Neutrino scattering off of nuclei within the Near and Far detectors are simulated by the NEUGEN [33] neutrino event generator. The NEUGEN simulation contains descriptions of the dominant neutrino interaction processes that can happen in the MINOS detectors. These are: quasi-elastic scattering $(\mathrm{QE})$, resonance production (RES), deep-inelastic scattering (DIS), and coherent pion production (Coh). The NEUGEN cross section predictions for some of these interaction processes along with data from a few different experiments used to tune NEUGEN are shown in Figure 5.7.

QE scattering is modeled, using the formalism discussed in Chapter 2, with the BBBA-2005 vector form factors and the dipole form for the axial-vector form factor. NEUGEN uses -1.267 for $F_{A}(0)$ and a nominal value of $0.99 \mathrm{GeV}$ for the axial-vector mass $M_{A}^{Q E}$. NEUGEN simulates the effect of the nucleus on the recoil proton using a RFG model, the RFG model includes the effects of Fermi motion along with Pauli blocking.

NEUGEN uses the Rein-Seghal [75] model of resonance production and a modified Bodek-Yang [76] model of DIS interactions. The Bodek-Yang model used by NEUGEN has been extended to improve the treatment of the resonance to DIS transition region [77]. For invariant masses less than $2.3 \mathrm{GeV}(W<2.3 \mathrm{GeV})$ a modified KNO [78] scaling is used to describe the multiplicity of the final state. How- 


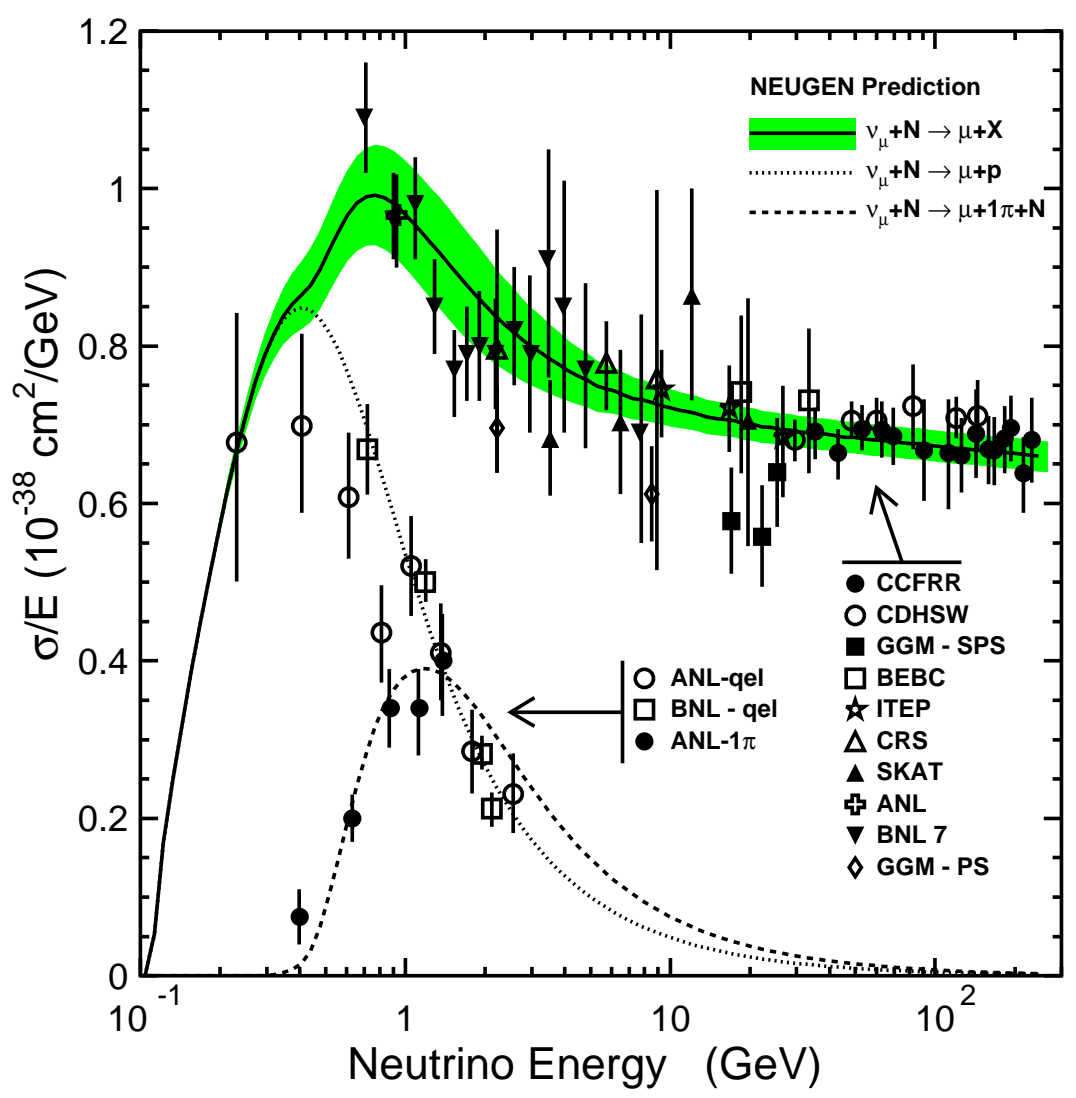

Figure 5.7: From [33][71]. The NEUGEN calculated cross sections for the $v_{\mu}$-CC inclusive, QE scattering and single pion production as a function of neutrino energy. These cross sections are calculated assuming an isoscalar averaged nucleon. The shaded band corresponds to the assumed uncertainty on the inclusive cross section. There is an assumed 3\% normalization uncertainty in the DIS cross section (defined as $W>1.7 \mathrm{GeV}$ ), there is an additional 10\% assumed normalization uncertainty in the QE and RES cross sections.

ever at higher invariant masses the PYTHIA/JETSET [79] model of hadronic showers is used. The transition from the KNO model to PYTHIA/JETSET is 
done gradually in the invariant mass region of $2.3<W<3.0 \mathrm{GeV}$ above which the PYTHIA/JETSET model is the sole model of hadronic showers.

The MINOS detectors and the most of the NuMI beamline use the GEANT-3 based program GMINOS. The simulation randomly samples the neutrinos that were saved at the FLUKA05/GNUMI step and propagates them through the MINOS detectors. Neutrino interactions are simulated in both physical detectors and the surrounding rock, the interaction products of neutrino interactions within the rock are propagated into the physical detectors. GMINOS is interfaced with NEUGEN, which generates the neutrino interactions. Final state interactions (FSIs) are modeled in NEUGEN using the INTRANUKE software package [80] [81]. INTRANUKE governs the passage of particles produced in the primary neutrinonucleon interaction through the internal structure of the nucleus. The final state particles generated by NEUGEN are passed back to GMINOS which propagates the particles through the detectors. Hadronic interactions are simulated using the GCALOR package. All of the simulated energy depositions are converted into light signals and electronic signals in the PMTs and front-end electronics. This simulated data is then passed to the MINOS reconstruction in an identical form to the MINOS data.

\subsubsection{Beam Re-Weighting}

Neutrinos are created as the result of the decay of pions and kaons within the NuMI decay pipe. Modeling the correct population of these secondary pions and kaons in the decay pipe, with the correct momentum is crucial for correctly modeling the neutrino energy spectrum. Model uncertainties in the production of these secondary hadrons generate significant uncertainty in the predicted neutrino flux. Furthermore comparisons between ND data and nominal ND MC simulations demonstrate significant disagreement in the high energy edge of the beam peak in the LE beam configuration. There is much less disagreement in the same energy range of other beam configurations. This is evidence that this discrepancy is not due to mis-modeling of detector acceptance or neutrino cross sections. A 
beam fitting procedure was implemented to further constrain the MC simulation, and thus produce a more accurate neutrino flux prediction [82].

The ND is used to measure the neutrino flux in several different beam configurations, each configuration has a corresponding MC simulation. Table 5.1 contains all of the different beam configurations used in the beam fit along with exposure of each configuration. A multi-variable fit of the MC simulations was performed until there was good agreement between the fit $\mathrm{MC}$ and the recorded ND data. Penalty terms within the fit were created to constrain the $\pi^{+} / \pi^{-}$ratio to the FLUKA0 5 MC simulation along with the NA49 hadron production experiment [83]. The fit was performed simultaneously on the different beam configurations, the best fit values were then used to calculate an importance weight for each simulated neutrino interaction.

\begin{tabular}{|l|c|c|c|c|}
\hline $\begin{array}{l}\text { Beam } \\
\text { Configuration }\end{array}$ & $\begin{array}{c}\text { Target } \\
\text { Position }(\mathrm{cm})\end{array}$ & $\begin{array}{c}\text { Horn } \\
\text { Current }(\mathrm{kA})\end{array}$ & $\begin{array}{c}\text { Peak } \\
E_{\mathrm{v}} \pm \text { r.m.s }(\mathrm{GeV})\end{array}$ & $\begin{array}{c}\text { Exposure } \\
10^{18} \mathrm{GeV}\end{array}$ \\
\hline \hline LE010/0kA & 10 & 0 & $7.4 \pm 4.1$ & 2.69 \\
LE010/170kA & 10 & 170 & $3.1 \pm 1.1$ & 1.34 \\
LE010/185kA & 10 & 185 & $3.3 \pm 1.1$ & 127.0 \\
LE010/200kA & 10 & 200 & $3.5 \pm 1.1$ & 1.26 \\
LE100/200kA & 100 & 200 & $5.6 \pm 1.5$ & 1.11 \\
LE250/200kA & 250 & 200 & $8.6 \pm 2.7$ & 1.55 \\
\hline
\end{tabular}

Table 5.1: The target position refers to the distance the target was displaced upstream of its default position inside the rst focusing horn. The peak (i.e., most probable) neutrino energy $E_{\mathrm{v}}$ is determined after multiplying the muon-neutrino ux predicted by the beam Monte Carlo simulation by charged-current cross-section. The r.m.s. refers to the root mean square of the peak of the neutrino energy distribution. The $0 \mathrm{kA}$ "horn-off" beam is unfocused and has a broad energy distribution.

The beam tuning procedure uses the BMPT parameterization [84] of hadron 
production. The BMPT parameterization is:

$$
\frac{d^{2} N}{d x_{F} d p_{T}}=\left[A\left(p_{z}\right)+B\left(p_{z}\right) p_{T}\right] \exp \left(-C\left(p_{z}\right) p_{T}^{3 / 2}\right)
$$

The functions $A\left(p_{z}\right), B\left(p_{z}\right), C\left(p_{z}\right)$ are each warped using a linear function of the fit parameters and $x_{F}$, where $x_{F}$ is the fraction of momentum in the longitudinal direction $\left(x_{F}=p_{z} / 120 \mathrm{GeV}\right)$. The linear warping of these functions are:

$$
\begin{aligned}
& A^{\prime}\left(x_{F}\right)=\left(p_{0}+p_{1} x_{F}\right) A\left(x_{F}\right) \\
& B^{\prime}\left(x_{F}\right)=\left(p_{2}+p_{3} x_{F}\right) B\left(x_{F}\right) \\
& C^{\prime}\left(x_{F}\right)=\left(p_{4}+p_{5} x_{F}\right) C\left(x_{F}\right)
\end{aligned}
$$

This procedure is the same for both $\pi^{+}$and $K^{+}$with the parameters $p_{0}$ through $p_{5}$ applying to $\pi^{+}$production and $p_{6}$ through $p_{1} 1$ applying to $K^{+}$production. The importance weight for positive pion and kaon production is given by:

$$
W\left(\pi^{+} / K^{+}, p_{T}, p_{z}\right)=\frac{\left(A^{\prime}+B^{\prime} p_{T}\right) \exp \left(-C^{\prime} p_{T}^{3 / 2}\right)}{\left(A+B p_{T}\right) \exp \left(-C p_{T}^{3 / 2}\right)}
$$

Two additional parameters are used to define a linear correlation between $v_{\mu}$ importance weights and $\bar{v}_{\mu}$ weights from $\pi^{-}$decay, as a function of $x_{F}$ and two more parameters for $\bar{v}_{\mu}$ from $K^{-}$decay. Which brings the total number of physics parameters to sixteen. All of the different ND data sets used for this beam fitting procedure are shown in Figure 5.8.

The beam tuning fit also includes six parameters that describe the effect of the beam optics uncertainties, along with effect that reconstruction and detector uncertainties have on the neutrino energy spectrum. These six additional parameters are constrained as nuisance parameters, three of these parameters account for beam optics effects which include uncertainties on the horn focussing, the error on the counting of the number of protons on target (POT), and scraping of the beam 


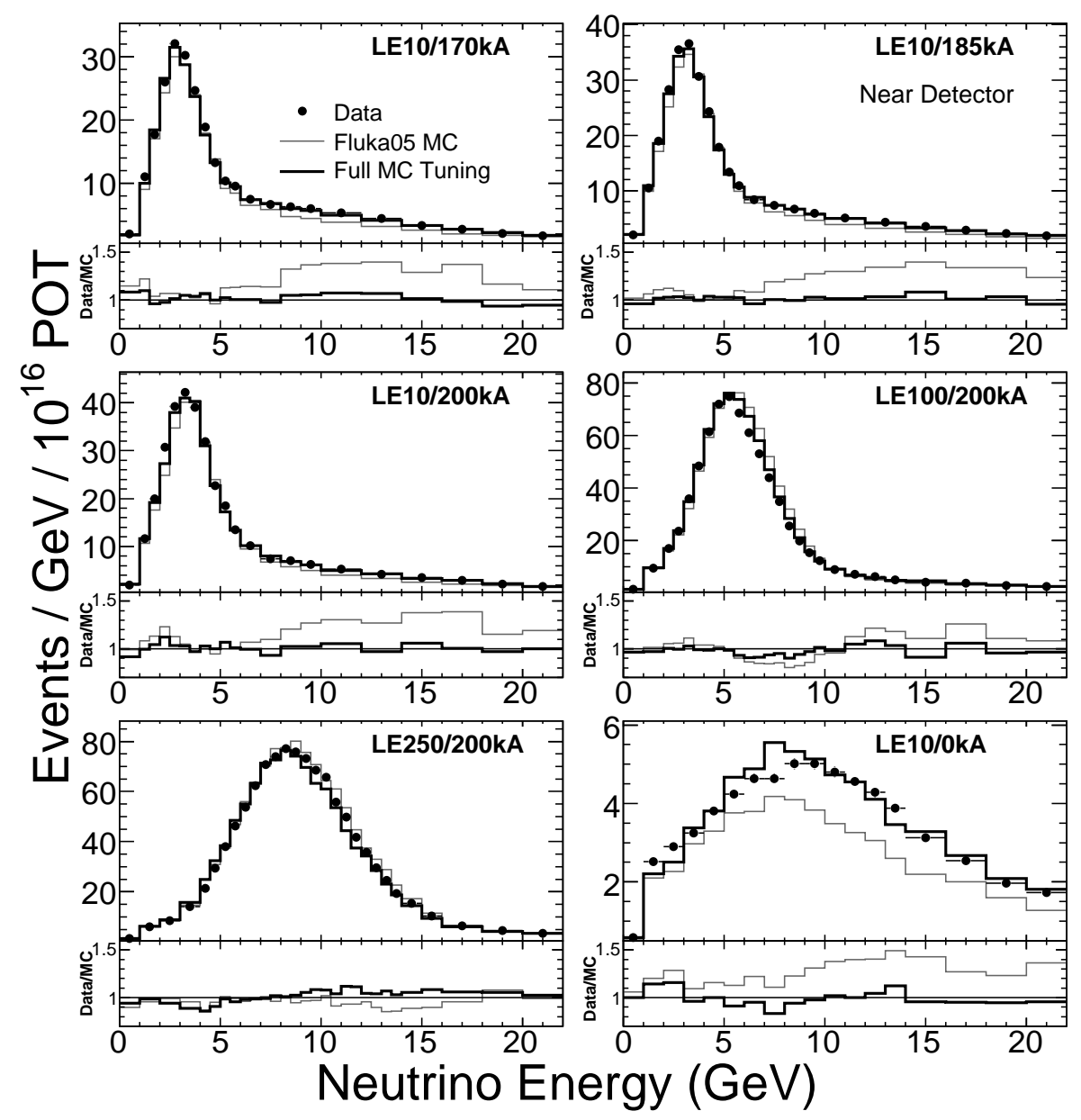

Figure 5.8: From [71]. $v_{\mu}$ charged-current energy spectra measured in the six beam configurations of Table 5.1 and compared with the Monte Carlo prediction. Two Monte Carlo predictions are shown: one (thin line) with the ab initio calculation based on FLUKA05, the other (thick line) after constraining hadron production, focusing and detector parameters with the neutrino data. Panels along the bottom of each figure show the ratio of the measured and simulated spectra.

along the collimator baffle. The contribution of these parameters to the predicted neutrino energy spectrum is shown in Figure 5.9. The remaining three parameters 


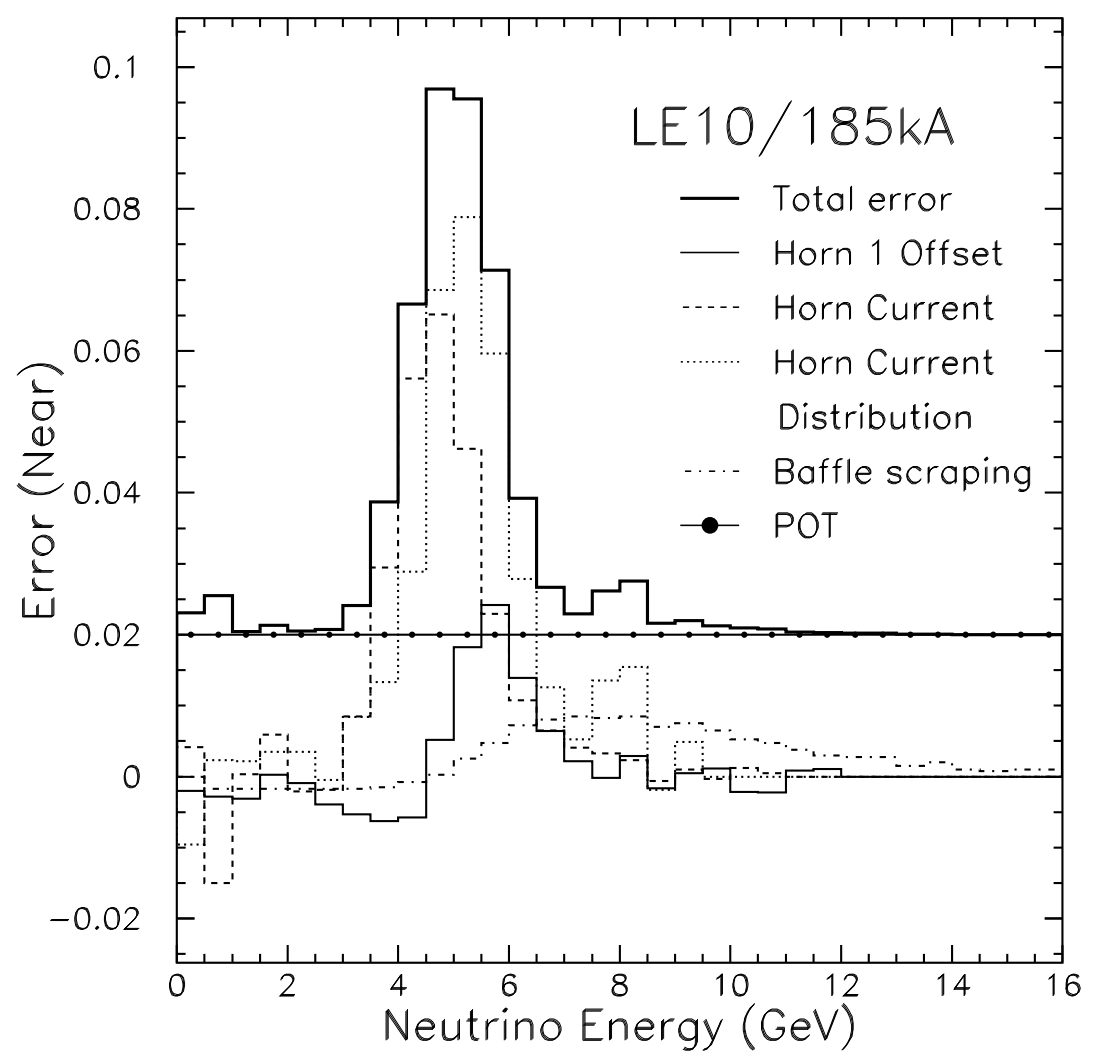

Figure 5.9: Figure From [71]. The error contribution to the predicted energy spectrum due to the current in the horn, the distribution of the horn current, offset in the horn, the scraping of the beam along the collimator baffle, and the counting of the protons on target as a function.

account for detector based effects, these parameters are included to ensure that the beam optics and hadron production parameters do not 'absorb' any real disagreement between data and MC that stems from physics based mis-modeling of either cross section or detector effects including reconstruction and calibration uncertainties. These final three parameters are a scale factor on the neutrino energy, an 
offset on the hadronic energy scale, and a scale factor on the true NC background that is naturally in the CC selected sample. 


\section{Chapter 6}

\section{Event Selection}

This analysis will measure the axial-vector mass $\left(M_{A}^{Q E}\right)$ by fitting the shape of the $Q^{2}$ distribution of QE dominated sub-samples of the $v_{\mu}$-CC event sample. Multiple sub-sample each with different kinematic compositions will be fit simultaneously, which will minimize the impact of important detector and physics based systematics to this analysis.

The event selection procedure is divided into several steps. First a $v_{\mu}$-CC enhanced (CC-like) event sample is selected. This CC-like event sample is divided into several different subsamples, these subsamples have different compositions of the interaction types (QE, RES, DIS).

\section{1 $v_{\mu}$-CC Event Selection}

The procedure for selecting $\mathrm{v}_{\mu}$-CC events is divided into several different subselection criteria. First a number of requirements are imposed that ensure that a candidate interaction comes from a high-quality neutrino beam in the proper configuration. Next additional selection criteria are imposed which ensure that the interaction originated from a beam neutrino and that all of the information necessary to properly reconstruct the interaction event was recorded within the detector. The final selection criteria ensure that the candidate interaction is a $v_{\mu^{-}}$ 
$\mathrm{CC}$ interaction, this includes a multivariate $v_{\mu}$-CC identification parameter.

\subsubsection{Beam Quality Criteria}

The first series of selection criteria are imposed on the data only (not on MC) these selection criteria ensure that a high-quality proton beam was delivered to $\mathrm{NuMI}$, and that NuMI is in the proper beam configuration. It is not necessary to apply these selection criteria to the MC because low quality proton beams are not simulated in the MC, and the various beam configurations used are simulated individually.

- Beam Configuration: Only data taken in the LE010185i beam configuration is used to measure the quasi-elastic axial mass parameter in this analysis. Data taken in the other beam configurations listed in Table 5.1 are not used to extract $M_{A}^{Q E}$ The data taken in the other beam configurations are only used for the beam tuning procedure (Section 5.2.1).

- Beam Spot Position: The position of the beam spot on the target is a good measure of the stability of the proton beam that is delivered to NuMI. The MC does not simulate neutrino interactions that originated from an offtarget beam, thus this cut will also ensure that $\mathrm{MC}$ and data originate from the beam position on the target.

- Horizontal and Vertical Beam Width: The horizontal and vertical beam width are also good measures of the quality of the proton beam delivered to NuMI. The MC does not simulate an increased or reduced beam radius, thus this selection criteria ensures that the data and MC originate from a beam with the same horizontal and vertical width.

- Horn Current: The amount of current through the focusing horns effects the neutrino energy spectrum seen by the ND, thus a check is made that the correct current is flowing through the horns. Thus selection criteria en- 
sures the interaction candidates in the data come from the same underlying neutrino flux.

There were $\sim 64$ million interaction candidates recorded in the MINOS near detector during the first MINOS run. Of these interaction candidates $\sim 54$ million pass the beam quality criteria. These selection criteria are applied to select a closer to ideal beam (the MC is generated using an ideal neutrino beam).

\subsubsection{Near Detector Event Quality Criteria:}

After the beam quality selections have been applied, additional selection criteria are applied to ensure quality data at the ND. These selection criteria ensure that the ND interaction candidate originated from a beam neutrino, while also ensuring that all of the necessary information to reconstruct the neutrino interaction is present.

- Coincidence with Spill Trigger: requiring that any candidate neutrino interactions occur in coincidence with the NuMI beam spill ensures that the majority of cosmic-ray induced muons that pass through the ND are removed. The spill trigger window is $16 \mu$ s wide starting $2 \mu$ s before the recorded spill trigger time and closing $14 \mu$ s after the spill trigger.

- Near Detector Magnetic Field: a check is made to ensure that the ND magnetic field coil is active. This selection criteria ensures that the information on the curvature of particle tracks is available for analysis.

- Fiducial Volume: the reconstructed event vertex must line within a $1 \mathrm{~m}$ diameter cylinder centered around the neutrino beam spot and $4 \mathrm{~m}$ along the $\mathrm{z}$-axis of the detector starting $1 \mathrm{~m}$ from the front face of the detector stopping $5 \mathrm{~m}$ inside the detector. The diameter of the cylinder ensures that for most neutrino interaction candidates the reconstructed hadronic shower is fully contained by the ND, and also that charged particles entering through the detector sides are rejected. 
There are $\sim 19$ million $\mathrm{MC}$ interaction candidates within the fiducial volume. $\sim 16$ million $(\sim 13$ million true $\mathrm{CC}, \sim 2$ million true CCQE) interaction candidates are reconstructed within the fiducial volume, of these interaction candidates $\sim 2$ million $(\sim 1.3$ million true $\mathrm{CC}, \sim 130$ thousand true $\mathrm{CCQE})$ did not actually truly interact within the fiducial volume. There are $\sim 5.6$ million data interaction candidates reconstructed in the fiducial volume.

\subsubsection{Charged Current Preselection}

After the ND event quality criteria are applied, several simple selection criteria are used to remove the easily identified NC background to the CC signal. These preselection criteria are:

- Presence of a Track: there must be at least one reconstructed track in the interaction candidate. This selection criteria removes a large portion of the NC background in the selected sample. The remaining NC interactions compose $\sim 15 \%$ of selected interaction candidates.

- Track Reconstruction Quality: the Kalman filter responsible for track finding and reconstruction quantifies the quality of the reconstructed track for any given interaction candidate. This track quality metric is based on the reduced $\chi^{2}$ from the fit of the particle's trajectory. This selection criteria requires that Kalman filter considers the track to be well reconstructed. The selection criteria also requires that the longitudinal difference in the track vertex position in the $\mathrm{U}$ and $\mathrm{V}$ views of the ND are different by no more than six planes. This selection criteria removes interaction candidates in which the bad track fit could cause the curvature measurement of the muon momentum to be very inaccurate. This criteria also removes interaction candidates where a mis-reconstructed track vertex can cause both estimates of the track momentum to be very inaccurate. Finally this criteria will also remove NC events where the reconstructed 'track' is in fact just a random collection of hadron shower hits. 
- Track Charge Sign: the Kalman filter's estimate of the track momentum from curvature is coupled to an estimate of the particles charge. Under the assumption that the track is produced by a muon, tracks with a 'negative' curvature are due to negatively charged particles. This selection criteria requires negative curvature, which will remove both NC interactions candidates with a positive pion track and interactions candidates that originate from $\bar{v}_{\mu}$-CC interaction.

After applying the charged current preselection criteria $~ 10.6$ million MC interaction candidates remain, of these $\sim 9.6$ million are true $\mathrm{CC}$ interactions and $\sim 1.5$ million are true CCQE interactions. $\sim 3.6$ million data interaction candidates pass the charged current preselection criteria.

\subsection{4 $v_{\mu}$-CC Multivariate Identification Parameter}

One of the criteria used to select for $v_{\mu}$-CC candidate interactions is a multivariate identification parameter. This identification parameter is calculated using a " $k$ Nearest-Neighbors" ( $\mathrm{kNN}$ ) algorithm. The kNN algorithm $v_{\mu}$-CC identification parameter [85] uses the values of $n$ reconstructed variables from a MC 'trainingsample' which contains both information about the reconstructed values and also information about the "true" interaction. This training sample is used to populate an $n$ dimensional space of reconstructed quantities that show good discrimination between charged current interactions and neutral current interactions. A data interaction candidate is placed within the $n$ dimensional space and compared to the $k$ nearest neighbors in the $n$ dimensional space. These $k$ nearest neighbors "vote" to classify the test interaction candidate, where the vote is based on the MC truth of the each of the $k$ nearest neighbors. Thus if the $\mathrm{kNN}$ output value is 0.90 , then $90 \%$ of the $k$ nearest neighbors are true $v_{\mu}$-CC interactions and voted to classify the test interaction candidate as a $v_{\mu}$-CC interaction and the remaining $10 \%$ of the $k$ nearest neighbors are true $\mathrm{NC}$ interactions and voted to classify the test interaction candidate as an $\mathrm{NC}$ interaction. The input variables along with the values 
$k$ and $n$ where tuned to maximize the product of selection purity and efficiency where purity and efficiency are defined as:

$$
\begin{aligned}
\text { purity } & =\frac{\# \text { True CC Events }}{\# \text { Selected Events }} \\
\text { efficiency } & =\frac{\# \text { Selected CC Events }}{\# \text { Total true CC Events }}
\end{aligned}
$$

the optimum value for $k$ was found to be 80 nearest neighbors, while the variables with the greatest distinguishing power are:

- Number of Planes in Interaction Candidate: $\mathrm{A} v_{\mu}-\mathrm{CC}$ interaction will produce a muon, muons usually have long tracks in the ND. However NC interactions will only produce a hadronic shower. Hadronic showers will, in general, not extend as far in the $z$-direction as far as muon track will. The data and MC distribution of this variable is shown in Figure 6.1a.

- Mean Energy Deposited per Strip: defined as the total pulse-height in an event, measured in MIP, divided by the total number of strips in the interaction candidate. Muons at MINOS energies are minimum-ionizing particles, and will have many strip hits with very little energy. Pions, which also interact strongly, will knock out protons as they propagate through the MINOS detectors. The knocked out protons are not minimum-ionizing particles and thus will deposit a lot of energy in each strip as the pions pass through. This variable will thus differentiate $\mathrm{NC}$ type interactions from $\mathrm{CC}$ interactions. The data and MC distributions for this variable are shown in Figure 6.1b.

- Signal Fluctuation Parameter: defined as the number of low pulse-height strips per high pulse-height strips in and around the track, where high and low pulse-height are defined relative to the average pulse-height of strips within the track. Pions are likely to be absorbed by a nucleus at the end pion track, while this is very rare for muons. The data and $\mathrm{MC}$ distributions for this variable are shown in Figure 6.1c. 

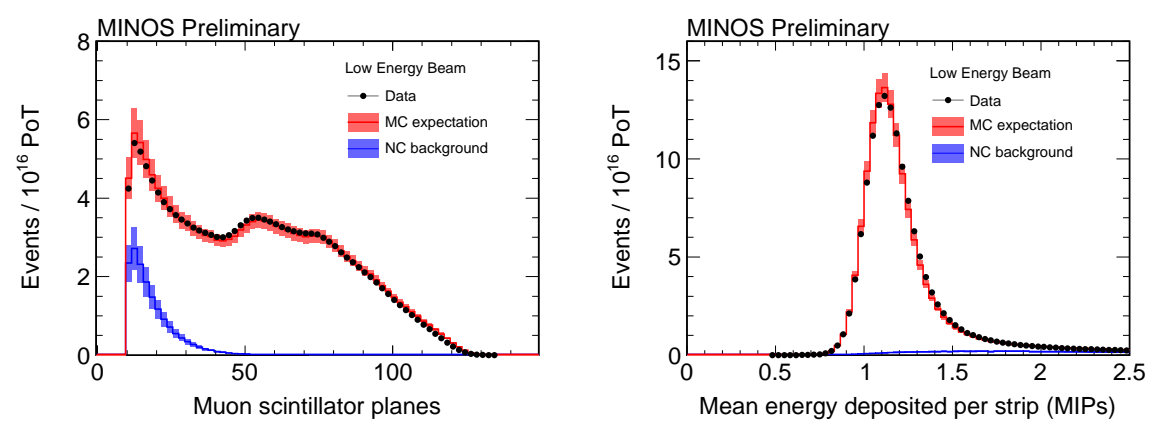

(a) Number of planes in $v_{\mu^{-}}$-CC interaction(b) Mean energy deposited per strip for $v_{\mu^{-}}$ candidates.

$\mathrm{CC}$ interaction candidates.
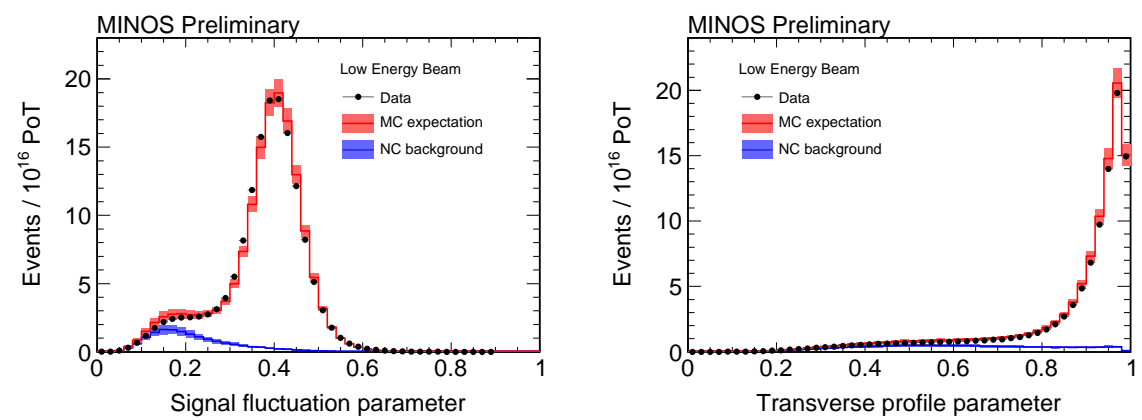

(c) Signal fluctuation parameter for $v_{\mu}$-CC(d) Transverse profile parameter for $v_{\mu}$-CC interaction candidates. interaction candidates.

Figure 6.1: Input parameters for the kNN multivariate identification parameter. Red: the MC prediction which includes the beam tuning reweighting described in Section 5.2.1. Blue: the NC contamination. Black Points: the data recorded in the LE010/185 beam configuration.

- Transverse Profile Parameter: defined as the pulse height of strips identified as track strips divided by the number of non-track strips near the track over threshold. This variable allows for the separation of the $\mu$-like track hadronic shower. Pion tracks will show fluctuations along the length of the track due to pion-nucleon rescattering. The data and MC distributions for this variable are shown in Figure 6.1d. 
The first $20 \%$ of the track is excluded from the calculation of these variables. This is done to remove most of the noise due to the hadronic shower of the interaction candidate. The data and MC distributions for the kNN multivariate identification variable along with are shown in Figure 6.2. The final $v_{\mu}$-CC selection criteria requires that the $\mathrm{kNN}$ multivariate identification variable be greater than 0.3. The final $v_{\mu}$-CC selection efficiency along with the NC contamination is shown in Figure 6.3. $\sim 3$ million data interaction candidates are selected by this multivariate identification parameter.

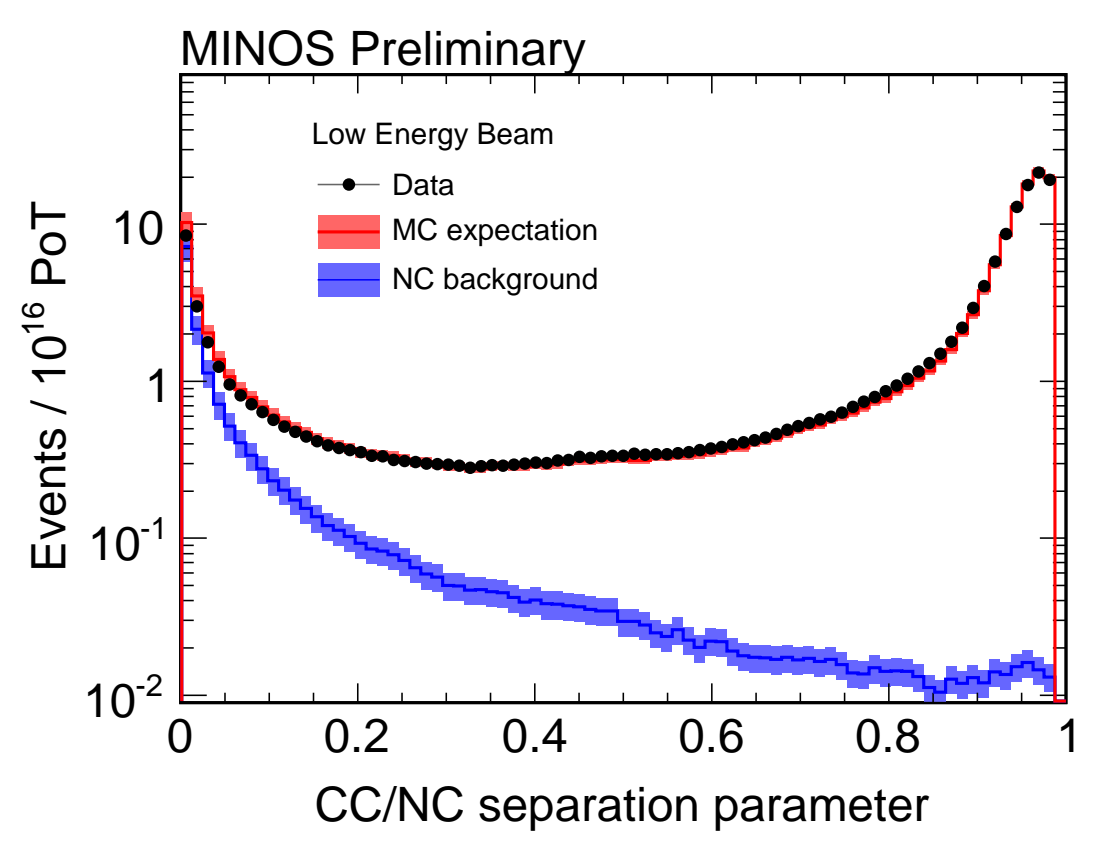

Figure 6.2: the kNN multivariate identification parameter for candidate $v_{\mu}$-CC interactions. Red: the MC prediction which includes the beam tuning reweighting described in Section 5.2.1. Blue: the NC contamination. Black Points: the data recorded in the LE010/185 beam configuration. 


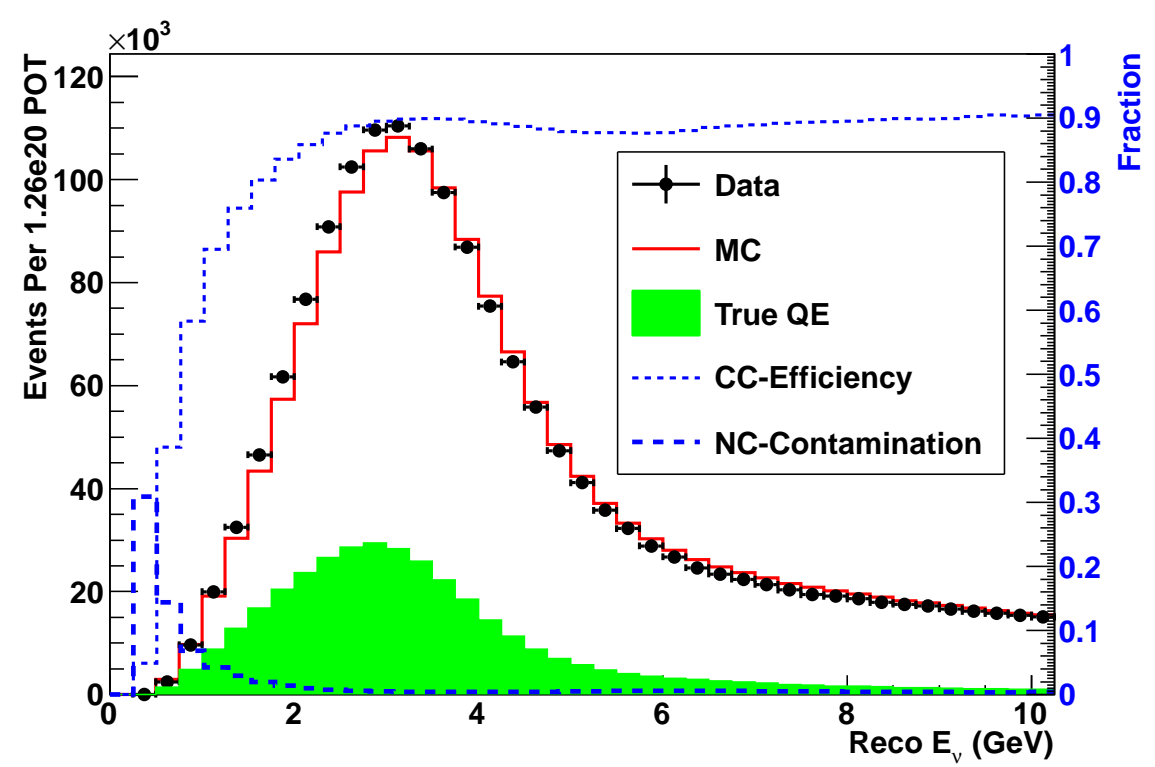

Figure 6.3: The $v_{\mu}$-CC energy spectrum of the MC (red), data (black), and QE-component (green) of the MC. The efficiency and NC contamination of interaction candidates selected by the multivariate identification parameter relative to the $\mathrm{CC}$ preselection are shown in blue with the $y$-axis on the right (also in blue).

\subsection{Partial Proton Track Reconstruction}

In MINOS the selection of charged current quasi-elastic (CCQE) like events is done in two stages. First a pre-selection stage that selects predominantly $v_{\mu} \mathrm{CC}$ like events, finally one of two QE-like selections loosely corresponding to one of the two QE detection channels. The CC pre-selection consists of a fiducial volume cut, track quality cut, track charge sign cut, and a muon PID. Both QElike selections require that there be exactly one track in the event.

There are two basic CC- $v_{\mu}$ QE final states. One possible CC- $v_{\mu}$ QE final state has muon and no visible recoil proton. It is possible that the recoil proton is not visible in the neutrino detector, because the recoil proton doesn't escape the nu- 
cleus or because the proton has energy below the energy threshold of the detector. The other CC- $v_{\mu}$ QE final state contains both a visible muon and recoil proton. This analysis will define two sub-samples of the $v_{\mu} \mathrm{CC}$ sample each composed primarily of one of these $\mathrm{CC}-\mathrm{v}_{\mu} \mathrm{QE}$ final states.

\subsubsection{Reconstruction Algorithm}

The standard MINOS reconstruction only reconstructs tracks that pass through 5 planes, which translates to a minimum proton kinetic energy of $2.5 \mathrm{GeV}$. The typical energy of a QE recoil proton is less than $2 \mathrm{GeV}$ figure (6.4), with most (95\%) of QE interactions having less than $1 \mathrm{GeV}$ this means that the standard MINOS reconstruction is unable to reconstruct the vast majority of recoil protons from QE interactions. To use the additional kinematic constraints from the proton to select QE-like interactions we have developed a new reconstruction algorithm to reconstruct the recoil proton. Reconstruction of the proton proceeds according to the following steps.

1. Reconstruct the primary muon track: This is done by the standard MINOS reconstruction.

2. Filter hits from the primary track: When attempting to reconstruct the potentially very short recoil proton tracks it is necessary to account for the possibility that the recoil proton may partially overlap with the reconstructed muon track. This is done by subtracting the ionization energy of the muon from the hits on the muon track. Any energy left in any given strip that remains could be from the recoil proton. In MINOS the average ionization energy of muons has been measured to be 600 sigcor, a quantity in units of raw ADCs where drift, linearity, and strip to strip corrections have been applied, but fiber attenuation which is a small effect has not.

3. Filter superfluous hits: In addition to filtering hits that are associated with the primary track, the algorithm applies two secondary hit filters. The first 


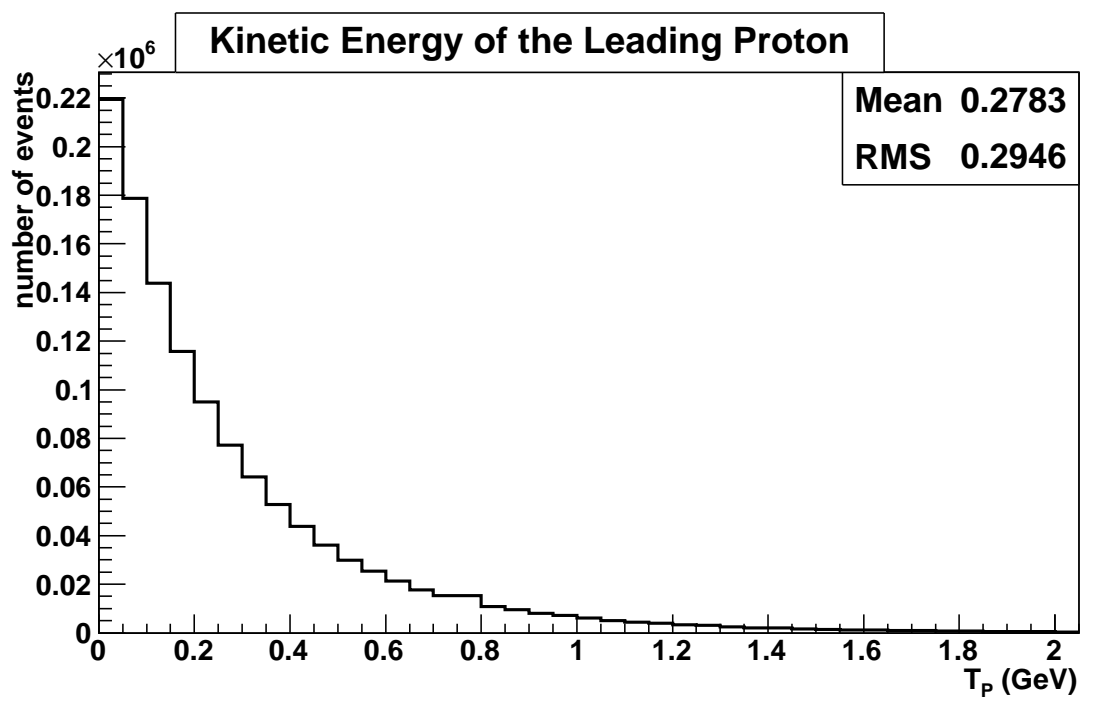

Figure 6.4: True kinetic energy of the leading proton in true QE events. The majority, $95 \%$, of the recoil have energy less than $1 \mathrm{GeV}$, nearly $99 \%$ have energy less than $2 \mathrm{GeV}$.

removes hits that are further than one meter down stream from the candidate interaction vertex which are very unlikely to be from a proton track because they are much too far away from the vertex to be caused by a proton interaction. The second filter removes very low energy hits, sigcor less than 150 , which are associated with photomultiplier tube crosstalk.

This analysis requires a full three-dimensional trajectory for the recoil proton, hence candidates proton tracks are required to have at least one hit in each view.

The algorithm for reconstructing the candidate proton track is illustrated in figure 6.5. Only hits that are within a box centered on the vertex, 20 planes long and 20 strips wide, are considered. These hits are grouped into candidate tracks by drawing a line between the center of each hit and the event vertex. The distance from the line to each other hit in the box is computed and the hit is included if the hit is further than one plane from the vertex and the distance is less than or equal to 

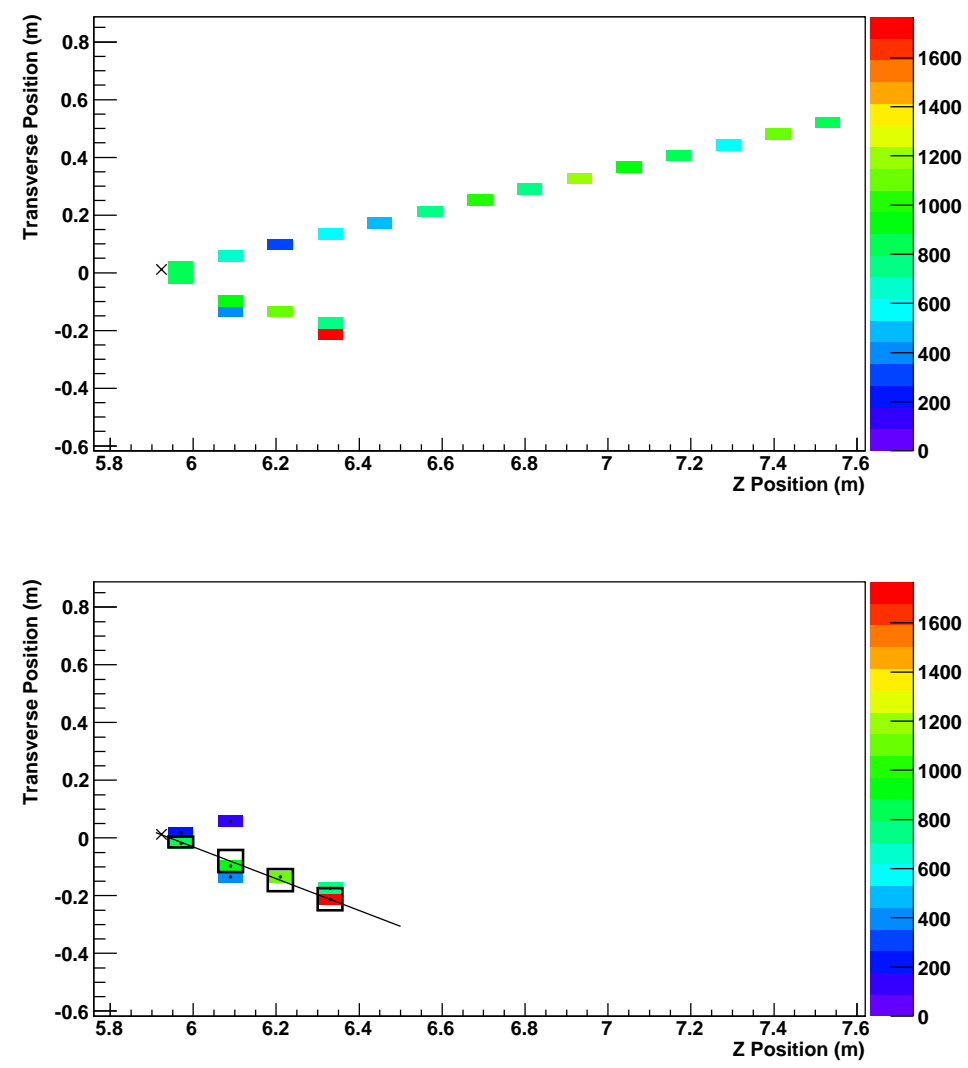

Figure 6.5: Event display of a characteristic true QE event in MINOS. On the top is the full event before any of the hit filters are applied. The plot on the bottom illustrates the grouping algorithm at work. In this case the line is drawn between the event vertex and the red hit that is in the candidate proton track furthest down stream from the event vertex. The black boxes represent the regions included in the grouping algorithm. Any hits whose hit centers are within one of the black boxes will be counted as part of the candidate proton track.

one strip width or the hit is within one plane and the distance is less than or equal to one half a strip width. The final track is the one that has the most hits. After the track has been formed within the 20 plane by 20 strip box, the 20 plane by 20 strip 
restriction is removed and the final line extended out to infinity accumulating hits that are within one strip. The difference between the reconstructed track direction and the true proton trajectory is shown in figure 6.6.
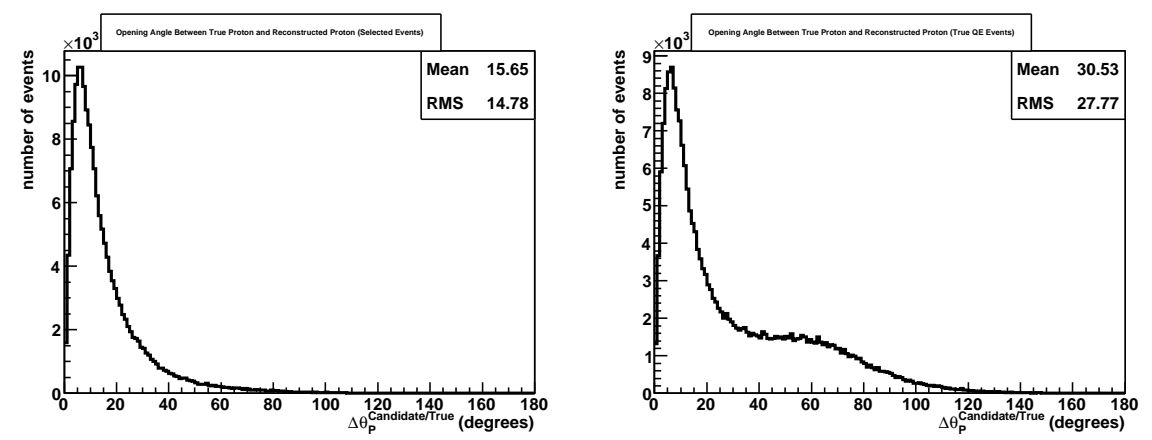

Figure 6.6: Left: The opening angle between the MC true proton momentum and the reconstructed proton trajectory for all two track $\mathrm{QE}$ selected events. Right: The opening angle between the MC true proton momentum and the reconstructed proton trajectory for all MC true CCQE events with a candidate proton track. The "shoulder" near $60^{\circ}$ in the plot on the right is due to incomplete removal of the muon track. When there is more than 600 sigcor of pulse height near the event vertex, and the true proton is below detection threshold, it is possible for this algorithm to get confused and interpret the remaining muon hits as coming from a proton. This effect is illustrated in Figure 6.5. In the bottom panel of Figure 6.5 there are clearly two hits that were left over from the muon track. It is these hits that could potentially confuse this algorithm and create this shoulder.

No attempt is made to reconstruct either the momentum or the energy of the candidate proton track, only the direction. In addition, the number of hits and their total pulse height, the total length of the candidate proton track, the number of planes between the highest pulse height and the furthest downstream hit in the proton candidate, and the number of unused hits in the vertex hadron shower with their total pulse height are also recorded. 
In $\mathrm{QE}$ interactions the recoil proton kinematics is known given the full muon kinematics and the neutrino beam direction. In this analysis, the reconstructed muon kinematics and the known beam direction are used to predict the recoil proton direction using a $\mathrm{QE}$ assumption and compared to the direction of the reconstructed candidate proton track. The comparison is done by calculating the opening angle, $\Delta \theta_{p}$, between these two vectors. True $\mathrm{QE}$ interactions should pile up near $\Delta \theta_{p}$ of zero. However due to detector resolution and solid angle effects the peak of this distribution for true QE interactions will be at low angle and not necessarily peaked at zero.

\subsection{The Interaction Sub-Sample Selections}

A preselection criteria is applied to the $v_{\mu}$-CC event sample. These preselection criteria remove obvious non quasi-elastic interactions such as DIS interactions with very large hadronic showers. This preselection criteria also removes all candidate interactions in which the muon track is not fully contained within the ND. These selection criteria are:

- Single Track: the number of reconstructed tracks in the interaction candidate is required to be exactly one. This selection criteria removes candidate interactions that are obviously not quasi-elastic, such as DIS interactions with very energetic hadron showers. Energetic hadron showers are more likely to have additional energetic particles that produce secondary muonlike tracks. The selection removes such events.

- Interaction Time Window: the high interaction rate at the MINOS ND can cause multiple neutrino interactions to overlap in the same region of the detector at the same time. When this happens it becomes very difficult separating the different interactions into different interaction candidates. The solution is to require that the separation in between different interaction candidates be greater than $70 \mathrm{~ns}$. 
- Stopping Muons: because the momentum resolution on muons that stop in the ND is better than the momentum resolution on muons that exit the detector. The resolution is better on stopping muons because the momentum can be measured from the track range which has better resolution than the momentum measured from curvature.

$\sim 2$ million data interaction candidates are retained by the sub-sample preselection criteria.

Once the preselection criteria has been applied the remaining interaction candidates are divided into four different sub-samples of interactions candidates. These sub-samples of the $v_{\mu}$-CC stopping muon sample are: the low hadronic energy quasi-elastic like sample, the two track quasi-elastic like sample, the two track background like sample, and an additional sub-sample which consists of interaction candidates that fail to be classified in either of the other sub-samples, the final event sub-sample is not used in this analysis. When discussing the properties of these sub-samples it is useful to define the interaction type efficiency and purity these are defined as:

$$
\begin{gathered}
\text { purity }=\frac{\text { \# True } \mathrm{I}_{\text {type }} \text { Events }}{\text { \# Selected Events }} \\
\text { efficiency }=\frac{\text { \# Selected True } \mathrm{I}_{\text {type }} \text { Events }}{\text { \# Total True } \mathrm{I}_{\text {type }} \text { Events In CC Sample }}
\end{gathered}
$$

where $\mathrm{I}_{\text {type }}$ is one of the possible interaction types (QE, RES, DIS, Coh).

\subsubsection{Low Hadronic Energy Quasi-Elastic Like Selection}

This selection is dominated by $v_{\mu}$-CC QE interactions with only muons in the final state. This sub-sample contains most of the true quasi-elastic interactions within the $v_{\mu}$-CC sample. However this sub-sample is also dominated by quasielastic interactions in the lower $Q^{2}$ region where nuclear effects dominate. This sample is primarily used to constrain the low $Q^{2}$ region behavior of the true quasielastic interactions, though this sub-sample does have some sensitivity to the $M_{A}^{Q E}$ parameter in the higher $Q^{2}$ region. The selection requirement for this sub-sample 
is:

- Reconstructed Hadronic Shower Energy: vertex shower energy less than $250 \mathrm{MeV}$. Three different types of neutrino interactions make up the majority of all the $v_{\mu}$-CC interactions these are the QE, RES, and DIS interactions (with a very small contribution form Coh). When considering these interactions at the neutrino generator level, they can be defined by considering the multiplicity of the final state, where QE interactions will produce just a single proton, RES interactions will produce a proton and a pion, and DIS interactions will potentially have many protons and pions. Thus QE interactions will leave the least amount of energy (on average) than these other interaction types. Figure 6.7 shows the reconstructed vertex shower energy. This Figure demonstrates the region below $250 \mathrm{MeV}$ is dominated by QE interactions.
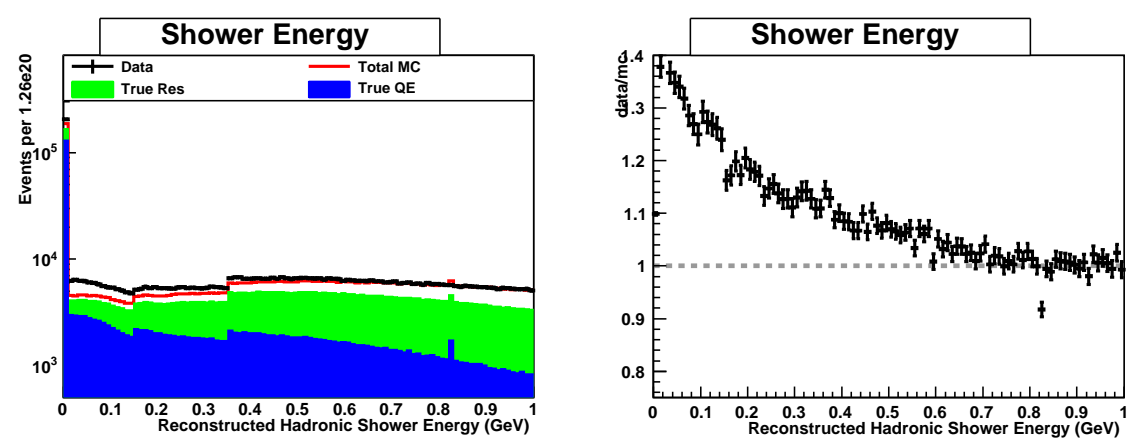

Figure 6.7: The reconstructed hadronic shower energy of the neutrino event. The histograms show the dominant NEUGEN interaction types. The event is flagged as QE like if the the reconstructed hadronic shower energy is less than $0.25 \mathrm{GeV}$ 


\subsubsection{Two Track Quasi-Elastic Like Selection}

This sub-sample is dominated by QE interactions with higher values of $Q^{2}$. This sub-sample has very little true $\mathrm{QE}$ interactions within the low $Q^{2}$ region, thus this sample has little sensitivity to the nuclear effects that dominate the low $Q^{2}$ region. This selection provides most of the sensitivity to the axial-vector mass parameter, due to higher QE purity of the sample at higher $Q^{2}$.

- Reconstructed Hadronic Shower Energy: vertex shower energy greater than $250 \mathrm{MeV}$. The intent of these sup-samples is to divide the preselected $v_{\mu}$-CC sample in several different exclusive sub-samples. This selection criteria ensures that there are no interaction candidates from the low hadronic energy QE-like selection.

- $\Delta \theta_{p}<30^{\circ}$

- Number of unused hits $<$ 6: Require that the number of hits in the vertex hadron shower that are not used in the candidate proton track must be less than 6. QE events should have very little vertex activity outside of the activity due to the muon track and the proton track.

- Energy profile consistent with proton: Require that the maximum pulse height hit of the candidate proton track be within one plane of the furthest downstream hit. Protons lose their energy very rapidly at the end of their track, this selection criteria looks for the signature of rapid energy loss at the end of the proton track.

\subsubsection{Two Track Background Like Selection}

This sub-sample is dominated by resonance interactions and is used to constrain the resonance background to the QE dominated sample. Even though this subsample is not dominated by $\mathrm{QE}$ interactions the $Q^{2}$ distribution is reconstructed using the QE assumption as Equation 2.38 instead of an alternative $Q^{2}$ calculation 
that may be more precise. The $\mathrm{QE}$ assumption $Q^{2}$ calculation is used because it is important to accurately model this mis-calculation of the non-QE background to the $\mathrm{QE}$ selections.

- Reconstructed Hadronic Shower Energy: vertex shower energy greater than $250 \mathrm{MeV}$. The intent of these sup-samples is to divide the preselected $v_{\mu}$-CC sample in several different exclusive sub-samples. This selection criteria ensures that there are no interaction candidates from the low hadronic energy QE-like selection.

- $\Delta \theta_{p}$ cut: if the number of unused hits is less than 6 , require that the trajectory of the proton predicted from muon kinematics be further than 30 degrees away from the candidate proton track. On occasion one of the $\Delta(1232)$ decay products is below the threshold of the detector. When this happens the number of unused hits may pass in to the range of the QE-like cuts. These events can be recovered by keep events previously removed by the QE-like $\Delta \theta_{p}$ cut.

- Number of unused hits < 13: require that the number of hits in the vertex hadronic shower that are not used in the candidate proton track be less than 13. The QE background is dominated by decays of $\Delta(1232)$ resonances. The $\Delta(1232)$ will decay to a nucleon and a pion, because the $\Delta(1232)$ decays to two particles, resonance events will leave additional hits in the vertex hadronic shower.

- Energy profile consistent with proton: Require that the maximum pulse height hit of the candidate proton track be within one plane of the furthest downstream hit. Protons lose their energy very rapidly at the end of their track, this cut looks for that signature. 


\subsubsection{Selection Purity and Efficiency}

There are three different event selections outlined above. Each enhances different interaction types, and with different kinematic characteristics. Because this analysis will be extracting a value for $M_{A}^{Q E}$ by simultaneously fitting the $Q^{2}$ distribution of all three of these selections, it is important to understand how well the different interaction types are enhanced within each of these event selections as well as understanding how efficiently the interaction types are selected, all as a function of $Q^{2}$. Figure 6.8 shows the fraction of true $\mathrm{QE}$ interactions in each of the selections and each selections efficiency at selecting true QE interactions. Figure 6.9 shows the fraction of true resonance interactions in each of the selections and each selections efficiency at selecting true resonance interactions.
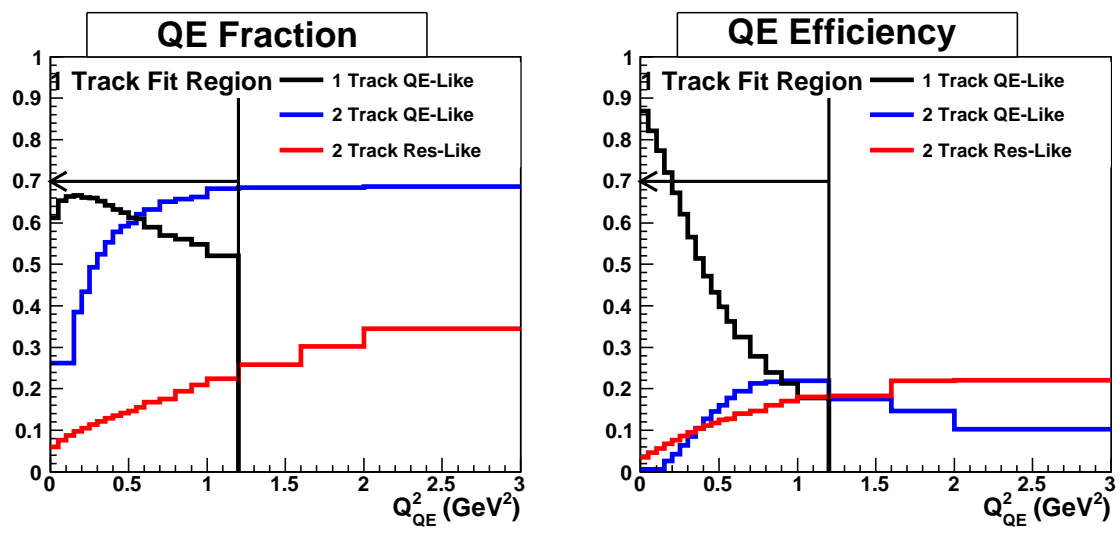

Figure 6.8: Left: The fraction of true $\mathrm{QE}$ interactions in the given selection. Right: The efficiency of selecting true $\mathrm{QE}$ interactions relative to the number of true $\mathrm{QE}$ interactions in the $\mathrm{v}_{\mu}$-CC selection. The one track QE selection is in black, the two track QE is blue, and the two track background selection is red. 

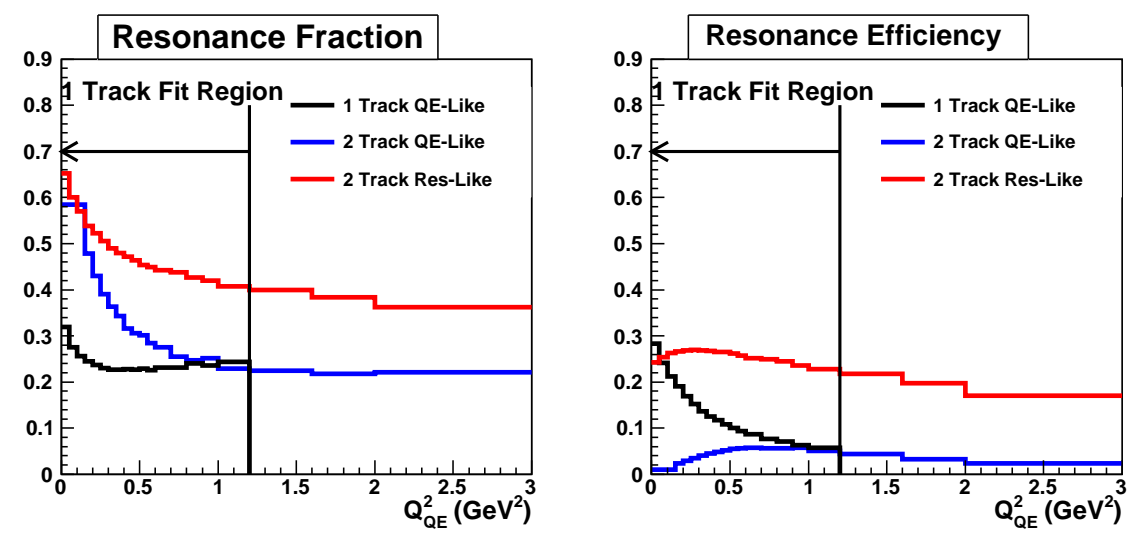

Figure 6.9: Left: The fraction of true resonance interactions in the given selection. Right: The efficiency of selecting true resonance interactions relative to the number of true resonance interactions in the $v_{\mu}$-CC selection. The one track QE selection is in black, the two track QE is blue, and the two track background selection is red.

\subsection{Comparisons of Data to Monte Carlo}

Because the threshold for reconstruction of proton candidates has been set at the absolute minimum to be considered a track, it is very important to check that the MC simulation is accurately modeling these low energy protons. This check is made by comparing reconstructed proton candidate quantities in the data to the same quantities in MC. Figures 6.10 through 6.12 are distributions of quantities that are not used in this analysis except for crosscheck purposes, while Figures 6.13 through 6.15 are for quantities that are used to select two track QE-like interaction candidates. When the plots show variables that are used for selecting one of the interaction sub-samples a selection based on the other selection variables is applied, thus the plots of the selection variables will show both the signal region and the sideband region with respect to that variable only. After examining these plots, some differences between the data and the $\mathrm{MC}$ become apparent. The two distributions that show the greatest data/MC differences are Figure 6.12 
which shows the distribution of the length of the candidate proton track and Figure 6.15 which shows the distribution of $\Delta \theta_{p}$. In Figure 6.6 the shoulder in the true $\mathrm{QE}$ distribution in the 60-80 degree range is due to the proton reconstruction algorithm finding the remains of the muon track instead of the recoil proton. This region is also the source of major disagreement between data and $\mathrm{MC}$. There are significantly more interaction candidates with excess energy very near the muon track in the data than in the MC. The physics implications of this data/MC difference is the topic of the Section 6.4.1. The data/MC differences in the track length could be due to strip to strip alignment; in the MC simulation the alignment is known perfectly and exactly matches the design, while real detector is aligned to $\pm 1 \mathrm{~mm}[86]$. 

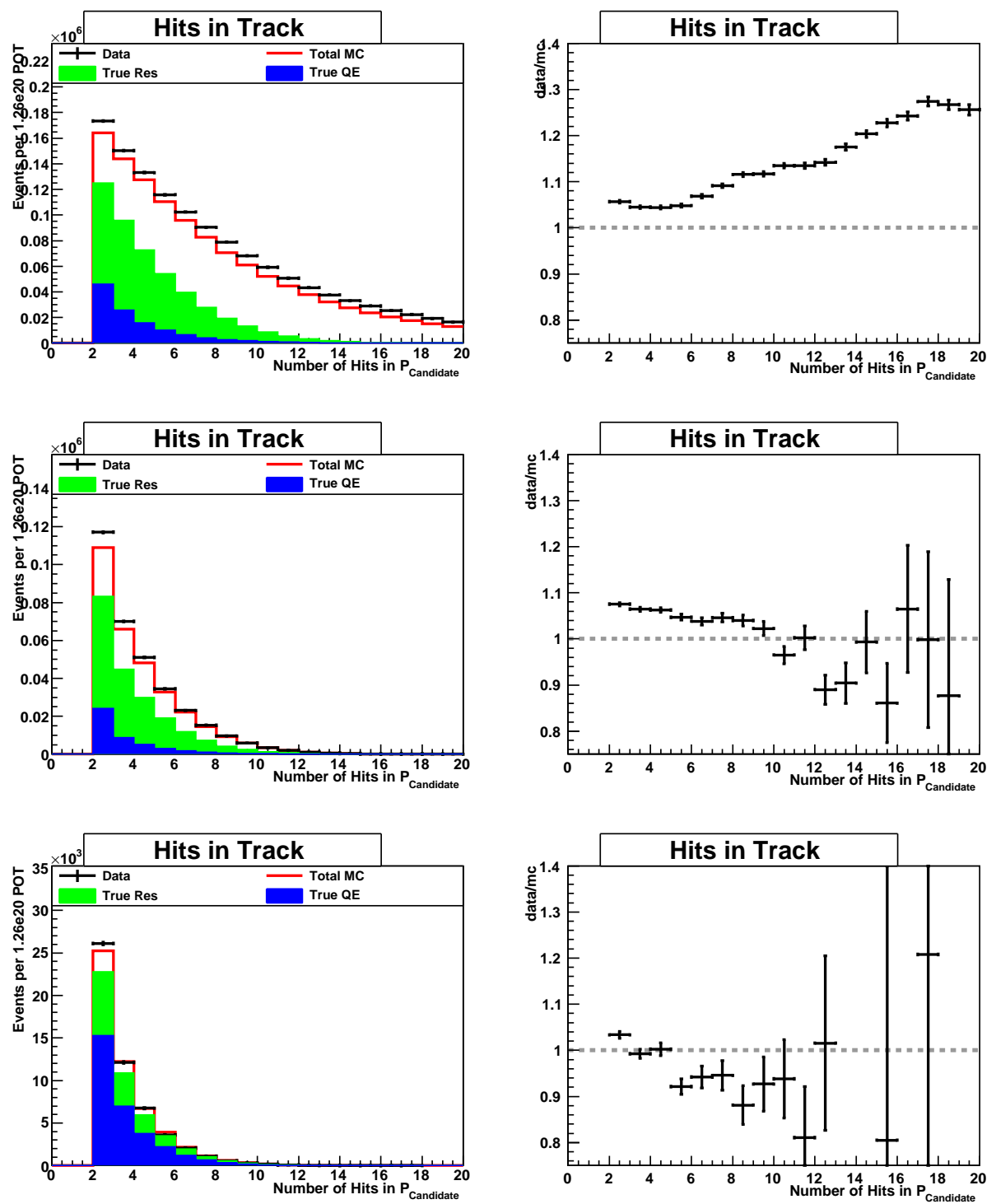

Figure 6.10: Number hits in the candidate proton track. Top: Preselected $\left(v_{\mu} \mathrm{CC}\right)$ events. Middle: Two track background selection. Bottom: Selected two track QE events. Left: Distributions with MC normalized to total data POT. Individual topological components of of the MC are shown as stacked histograms. Right: Ratio of data to MC. 

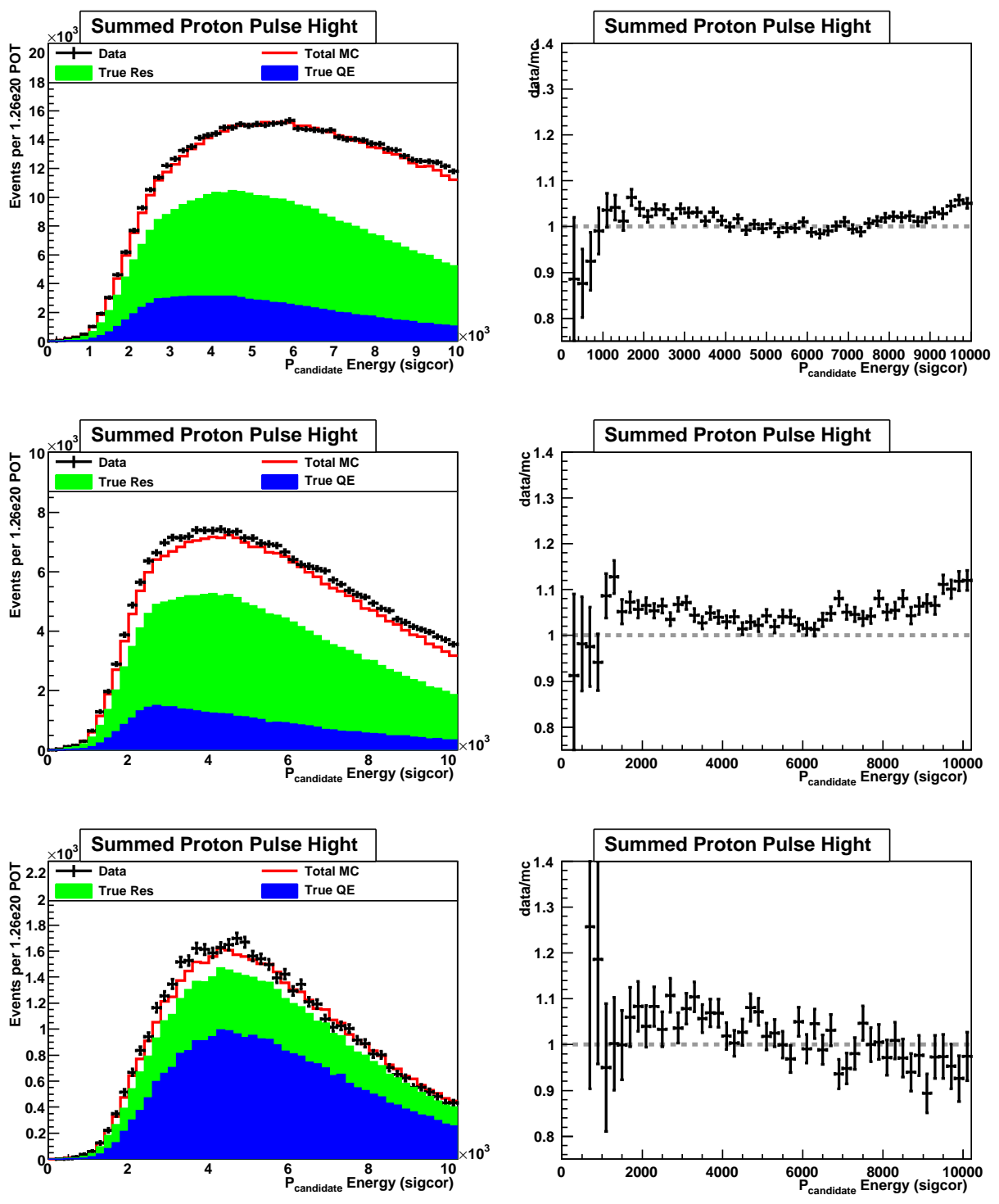

Figure 6.11: Summed pulse height of the scintillator strips in the candidate proton track. Top: Pre-selected $\left(\mathrm{v}_{\mu} \mathrm{CC}\right)$ events. Middle: Two track background selection.Bottom: Selected two track QE events. Left: Distributions with MC normalized to total data POT. Individual topological components of of the MC are shown as stacked histograms. Right: Ratio of data to MC. 

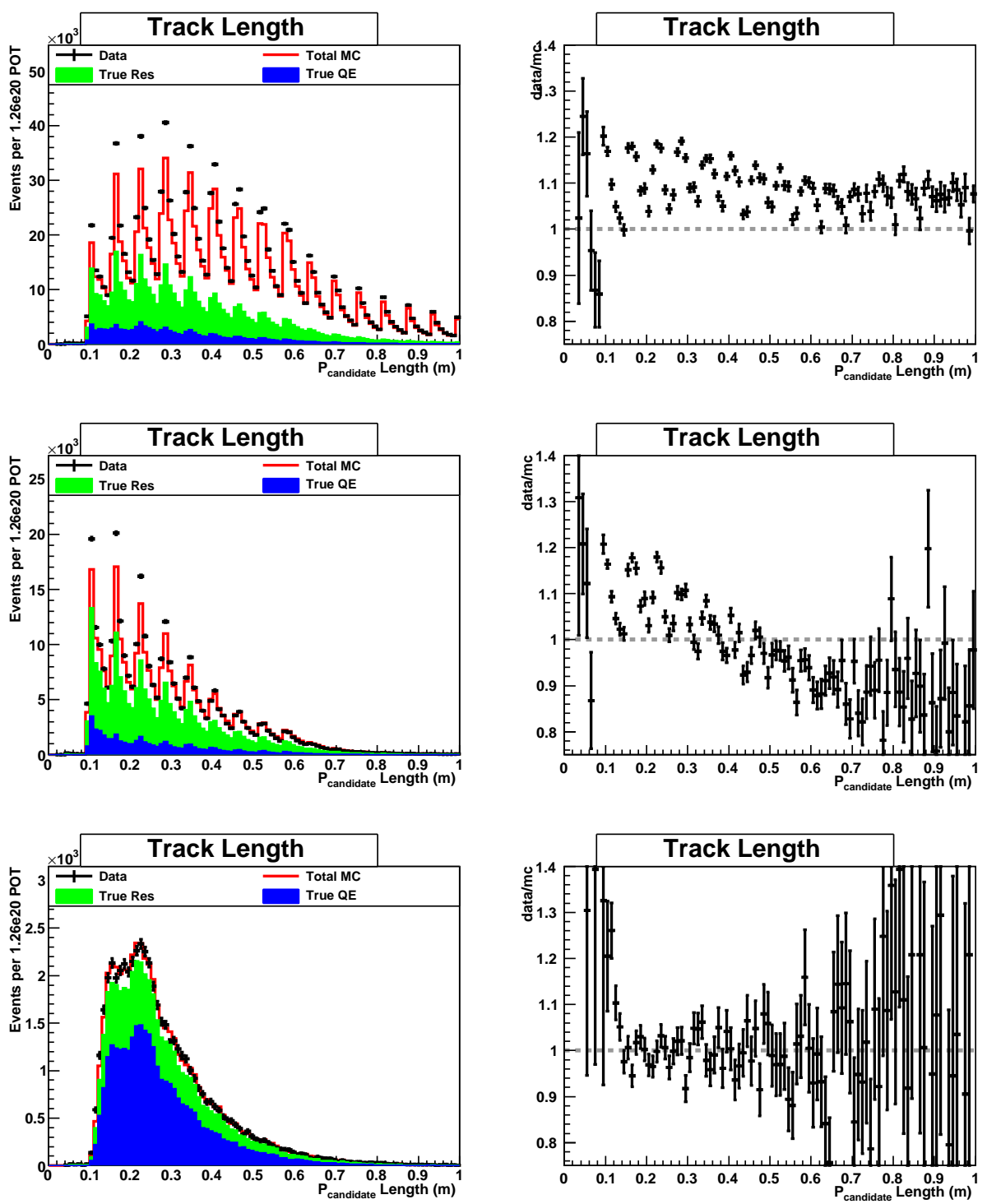

Figure 6.12: Total path length of the candidate proton track. Top: Preselected $\left(v_{\mu} \mathrm{CC}\right)$ events. Middle: Two track background selection. Bottom: Selected two track QE events. Left: Distributions with MC normalized to total data POT. Individual topological components of of the MC are shown as stacked histograms. Right: Ratio of data to $\mathrm{MC}$. 

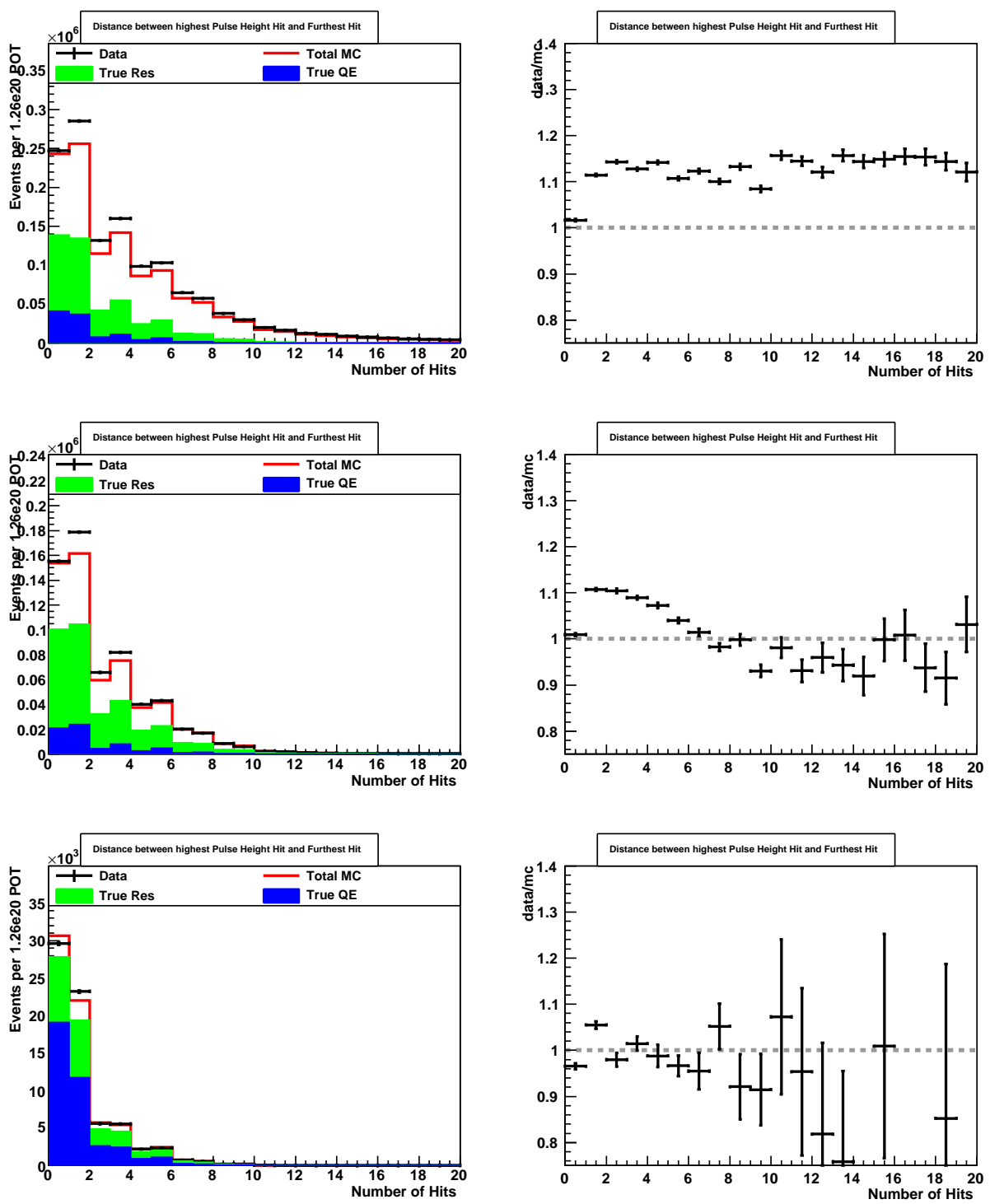

Figure 6.13: Distance between maximum pulse height hit and furthest downstream hit. Top: Pre-selected $\left(v_{\mu} \mathrm{CC}\right)$ events. Middle: Two track background selection. Bottom: Selected two track QE events. Left: Distributions with MC normalized to total data POT. Individual topological components of of the MC are shown as stacked histograms. Right: Ratio of data to MC. 

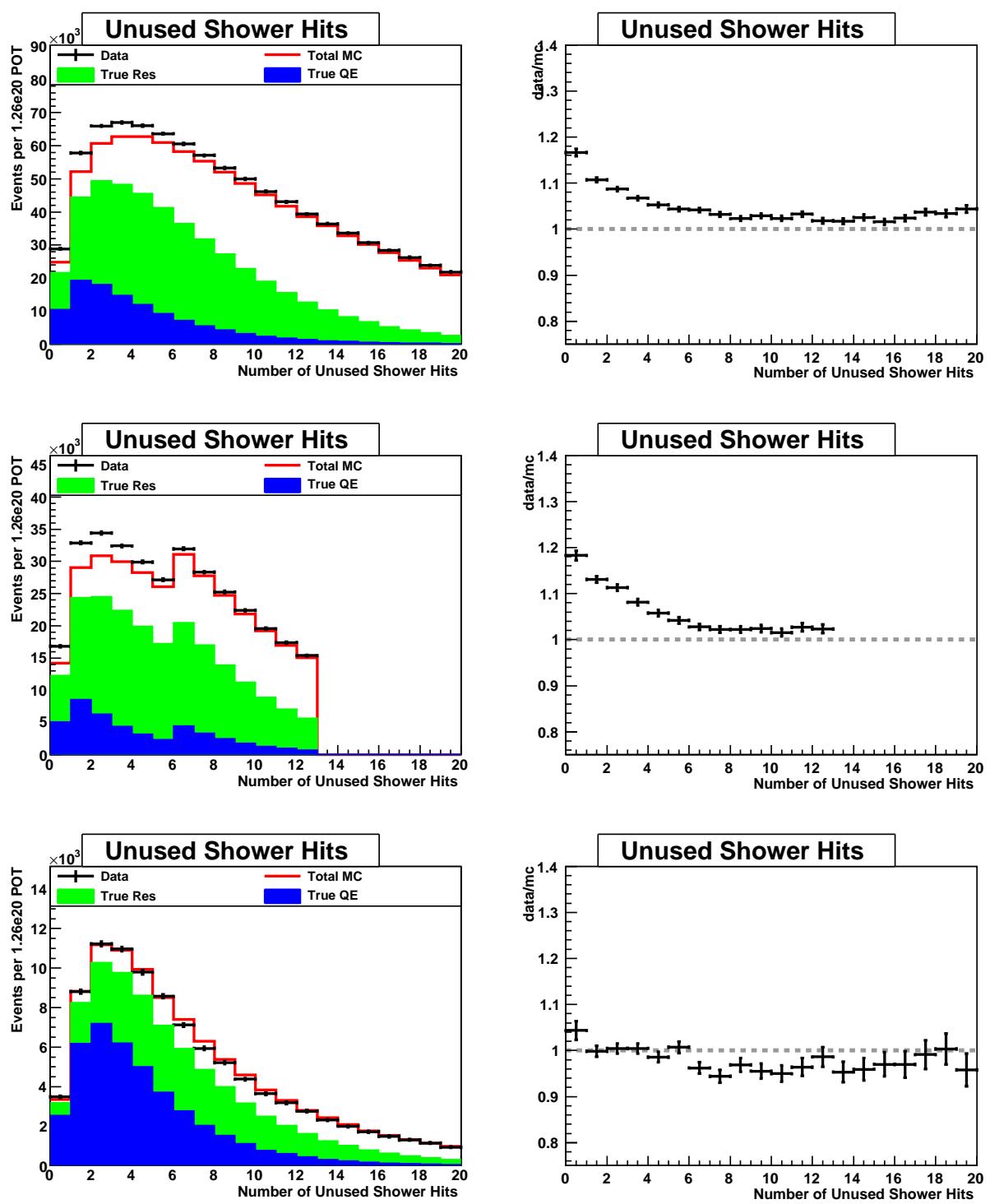

Figure 6.14: Number of unused hits in the vertex hadronic shower. Top: Pre-selected $\left(v_{\mu} \mathrm{CC}\right)$ events. Middle: Two track background selection. Bottom: Selected two track QE events. Left: Distributions with MC normalized to total data POT. Individual topological components of of the MC are shown as stacked histograms. Right: Ratio of data to MC. 

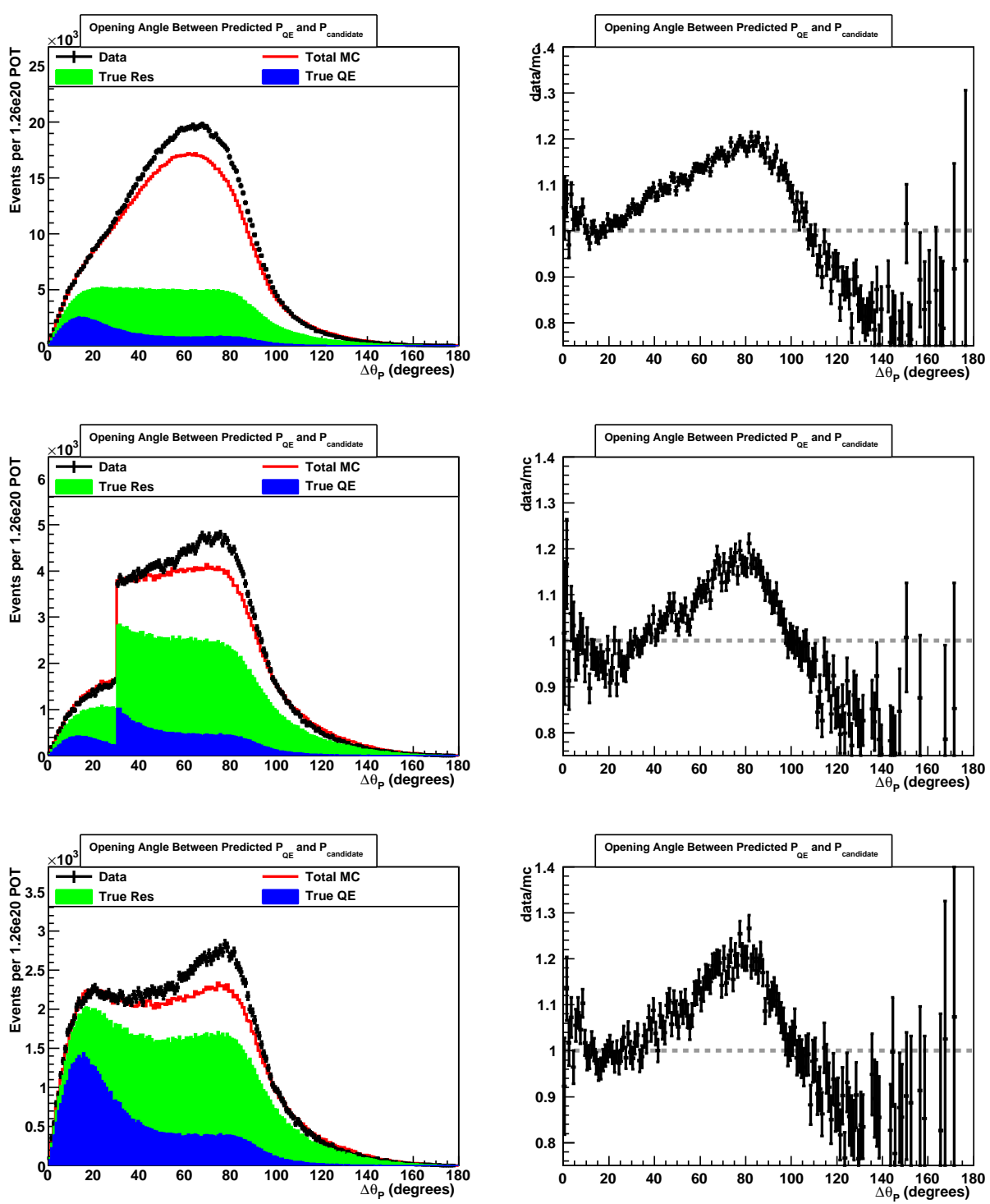

Figure 6.15: Opening angle between the candidate proton track and the proton trajectory predicted from muon kinematics $\left(\Delta \theta_{p}\right)$. Top: Pre-selected $\left(v_{\mu} C C\right)$ events. Middle: Two track background selection. Bottom: Selected two track QE events. Left: Distributions with MC normalized to total data POT. Individual topological components of of the MC are shown as stacked histograms. Right: Ratio of data to $\mathrm{MC}$. 


\subsubsection{Nuclear Correlations in MINOS}

MINOS is a coarse grained tracking detector, but as has been shown earlier in this document MINOS has the ability to partially reconstruct the recoil proton in $v_{\mu}$ CCQE interactions. However, the secondary nucleon of an SRC type interaction is unlikely to be reconstructable in MINOS, because the secondary nucleon has a much lower energy than the primary recoil nucleon. While it isn't possible to identify SRC type interactions on an event by event basis in MINOS, we can look for differences between data and the MC prediction of distributions that describe the activity in the first few strips near the event vertex which would be impacted by the presence of SRC.

In the $\mathrm{QE}$ selected plots of $\Delta \theta_{p}$ in Figure 6.15 there is a deficit in the $\mathrm{MC}$ compared to the data in the 60 to 90 degree region. This region is where the proton finding algorithm picks up the remnants of the muon track instead of the proton track. This deficit in the MC prediction implies that the muon track is not removed as cleanly in the data as it is in the MC. This deficit is because an occasional event has excess energy very near the muon that doesn't get subtracted from the muon track when the muon hits are filtered out of the event. The excess energy near the vertex may be due to the energy of the secondary nucleon from an SRC type interaction. If the excess energy near the muon track is due to an extra nucleon from an SRC type interaction than we should see a difference in the shape between the data and the MC in the total energy that isn't accounted for in either the candidate proton track or the muon track. Under an SRC assumption events with $\Delta \theta_{p}$ greater than $30^{\circ}$ should have large amounts of excess energy which is not accounted for in either the muon track or the proton track. This excess energy would not appear in the $\Delta \theta_{p}$ less than $30^{\circ}$ sample. The distribution of the excess energy is shown in Figure 6.16 where the MC exhibits a deficit relative to the data consistent with the pattern suggested by SRC. The MC deficit extends up to 2000 sigcor (3.2 MIPs) which is a substantial amount of energy. It seems unlikely that this could be due mis-modeled detector effects. 

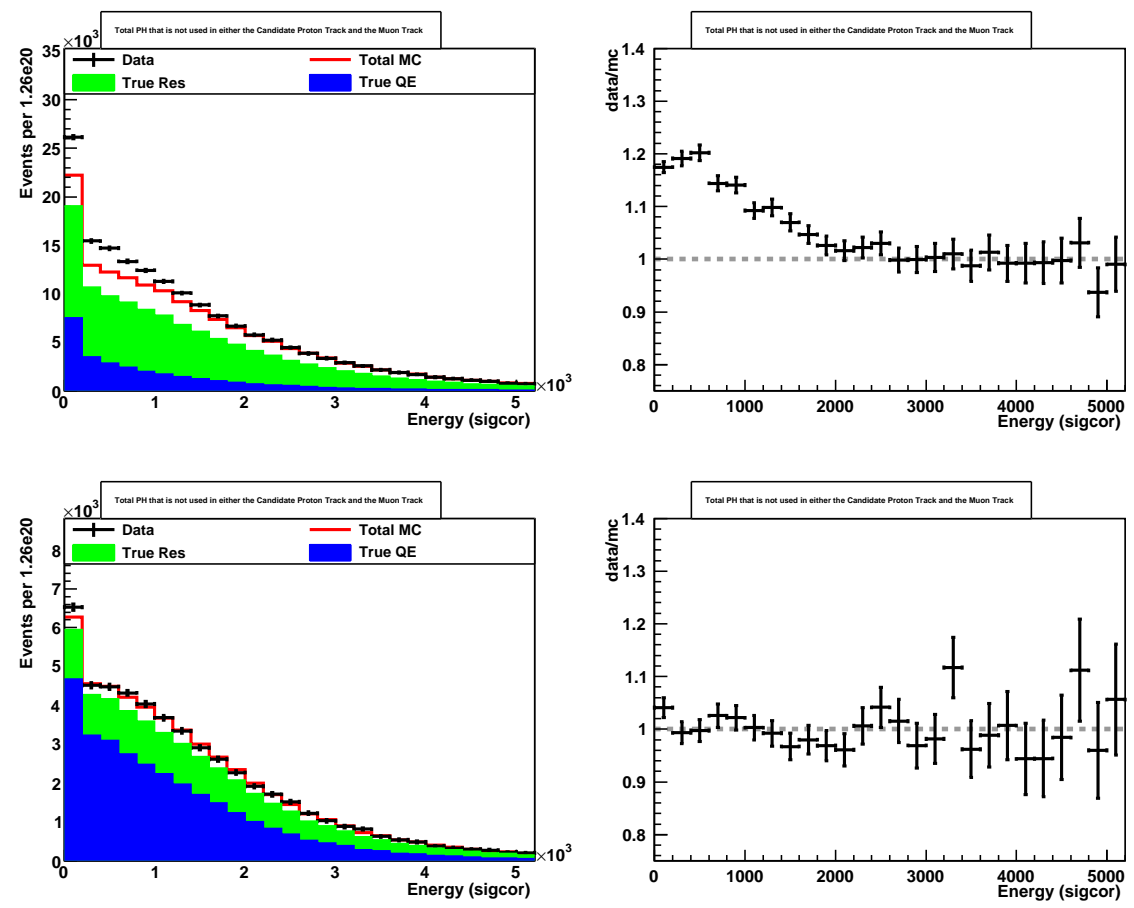

Figure 6.16: Summed pulse height in sigcor of all of the hits that are not used in either the muon track or the candidate proton track. Top: $\Delta \theta_{p}$ sideband selections (QE-like except $\Delta \theta_{p}>30$ ). Bottom: Selected two track QE events. Left: Distributions with MC normalized to total data POT. Individual topological components of of the $\mathrm{MC}$ are shown as stacked histograms. Right: Ratio of data to MC. The MC deficit in the 0-2000 sigcor region of the top plots when considered. along side the MC deficit in the 60-90 degree region of Figure 6.15 is further evidence of a secondary nucleon from an SRC type interaction. The extra energy that we are seeing is substantial enough that additional quanta seems like a more likely explanation than a mismodeling of detector effects 


\section{Chapter 7}

\section{Extracting the Axial-Vector Mass}

The MINOS CCQE-like sub-samples have a significant contribution from nonCCQE like interactions, this is unlike the NOMAD and MiniBooNE measurements of $M_{A}^{Q E}$ both of which have a smaller contribution from non-CCQE interactions in their CCQE-like sub-samples. This is further complicated because the MINOS non-CCQE sub-samples have a significant contribution from CCQE interactions. Thus characterizing the background must be done simultaneously with any measurement of $M_{A}^{Q E}$. First a Mock Data study was done to demonstrate the validity and quantify the improvements of a multi sub-sample fit procedure, this study is presented in Section 7.2. Sections 7.3 and 7.4 document attempted fit procedures that where unable to simultaneously describe the QE-like sub-samples and the background-like sub-samples. Finally Section 7.5 implements a method for simultaneously constraining the background while fitting for the $M_{A}^{Q E}$ parameter, the fit procedure outlined in Section 7.5 is the procedure used to calculate the results presented in this dissertation.

\subsection{Fitting Procedure and Fit Parameters}

The three interaction candidate sub-samples discussed in Section 6.3 are fit simultaneously in bins of $Q_{Q E}^{2}$. Each selection is binned in a slightly different manner. 
The low hadronic energy sub-sample is divided into 18 bins over the $Q_{Q E}^{2}$ range of $0<Q_{Q E}^{2}<1.2 \mathrm{GeV}^{2}$. The two track $\mathrm{QE}$ sub-sample divided into 20 bins over the $Q_{Q E}^{2}$ range of $0<Q_{Q E}^{2}<3.0 \mathrm{GeV}^{2}$, and the two track background subsample is divided into 21 bins also over the $Q_{Q E}^{2}$ of $0<Q_{Q E}^{2}<3.0 \mathrm{GeV}^{2}$. The expected number of interaction candidates for each bin is calculated from the MC for different values of the $M_{A}^{Q E}$ parameter along with some other systematic error parameters. A modified $\chi^{2}$ is performed by minimizing the $\chi^{2}$ function:

$$
\chi_{M C \text { stats }}^{2}=\sum_{i=1}^{n B i n s} \frac{\left(o_{i}-e_{i}\left(\alpha_{1}, \ldots, \alpha_{N}\right)\right)^{2}}{o_{i}+S e_{i}\left(\alpha_{1}, \ldots, \alpha_{N}\right)}+\sum_{j=2}^{N} \frac{\Delta \alpha_{j}^{2}}{\sigma_{\alpha_{j}}^{2}}
$$

In which $o_{i}$ is the number of observed events in mock data for bin i, $e_{i}\left(\alpha_{1}, \ldots, \alpha_{N}\right)$ is the expected number of events in the Monte Carlo for bin i given the fit parameters, $S$ is the scale factor applied to normalize the MC histogram and the penalty term is computed with $\Delta \alpha_{j}$, the shift from nominal for the $j^{\text {th }}$ systematic parameter, and $\sigma_{\alpha_{j}}$, the assumed $1 \sigma$ error on the $j^{\text {th }}$ systematic parameter. The first fit parameter $\alpha_{1} \equiv M_{A}^{Q E}$ and is treated as a free parameter in the fit and does not influence the penalty term.

In addition to considering $M_{A}^{Q E}$, the fit also includes some of the following four systematic parameters that are considered to be the most dominant for a shapeonly fit to $Q_{Q E}^{2}$ : The effect of a $\pm 30 \%$ shift to $M_{A}^{Q E}$ parameter in the two track QE-like sample is shown in Figure 7.1

- Muon energy scale: The muon energy scale is the most prominent systematic error when fitting for $M_{A}^{Q E}$ and it directly affects the calculation of $Q_{Q E}^{2}$. Given that the uncertainty on the range-based momentum of muons that stop in the Near Detector is smaller than that for the curvature-based momentum of muons that exit, only stopping muons are included when fitting for $M_{A}^{Q E}$. This does not result in a large decrease in QE-like sample statistics because there are relatively fewer quasielastic interactions in events with higher energy muons. As such, the assumed one sigma uncertainty in the muon energy for stopping muons is taken to be $2 \%$ for the 

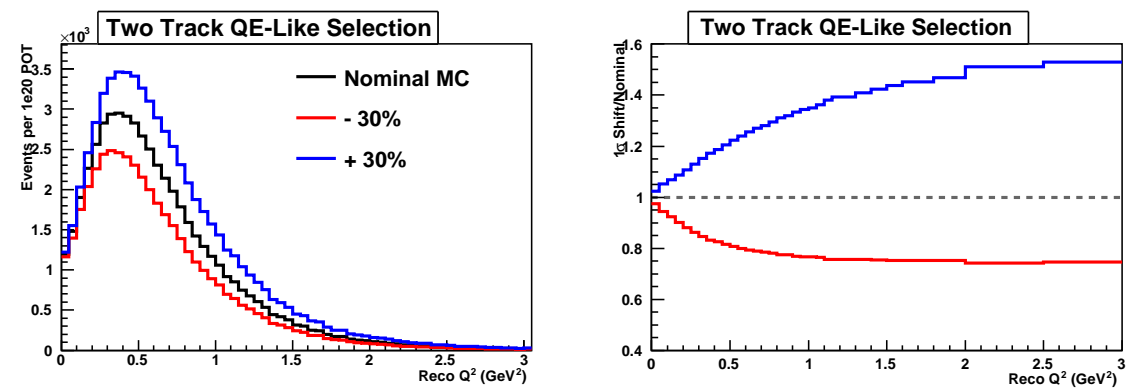

Figure 7.1: Comparison of the nominal MC to reweighted MC with $\pm 30 \%$ changes to the value of $M_{A}^{Q E}$. Only stopping muon events are included in this figure.

purposes of calculating the $\chi^{2}$ penalty term. This uncertainty is driven by the uncertainty in the thickness of the steel planes in the MINOS detectors along with the uncertainty in the final stopping location of the muon.The effect of $\pm 2 \%$ changes to the muon energy scale on the $Q_{Q E}^{2}$ distribution for the 1 track QE-like sample are illustrated in Figure 7.2.
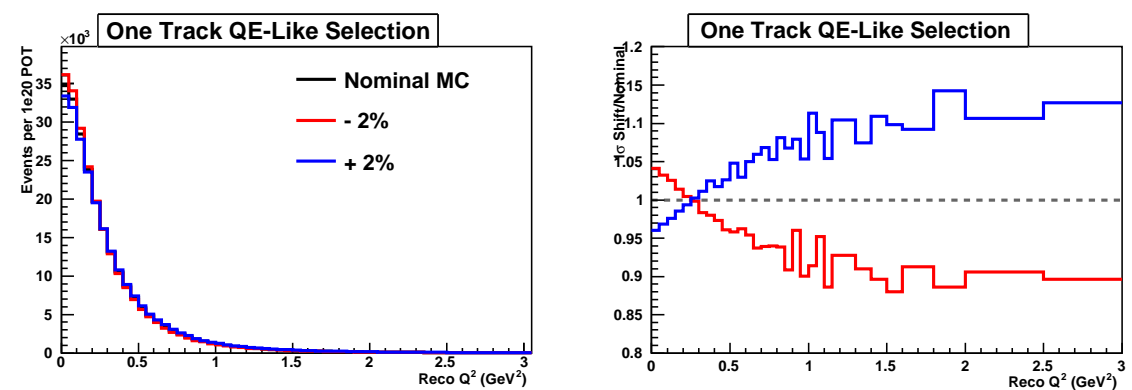

Figure 7.2: Comparison of the nominal MC to scaled MC with $\pm 2 \%$ scalings of the muon energy. Only stopping muon events are included in this figure.

- $\boldsymbol{M}_{A}^{R E S}$ : This parameter plays a similar role to $M_{A}^{Q E}$ but in the modeling of resonant interactions. Changes to $M_{A}^{R E S}$ will affect both the shape and rate 
of the resonance production cross section as a function of $Q^{2}$. Resonance events form the dominant background in the QE-like sample (although the extent to which they can be labeled a background is arguable given that they are very kinematically similar to the true quasielastic events) and this parameter assures that the fit does not give a biased $M_{\tilde{A}}^{Q E}$ value due to the mis-modeling of these events. The standard uncertainty of $15 \%$ is used for this parameter when calculating the penalty term $\chi^{2}$ penalty term. This value for the uncertainty is driven by the from a global analysis of the resonances axial-vector mass. Figure 7.3 shows the effect of changes to this parameter on the 1 track QE-like sample.
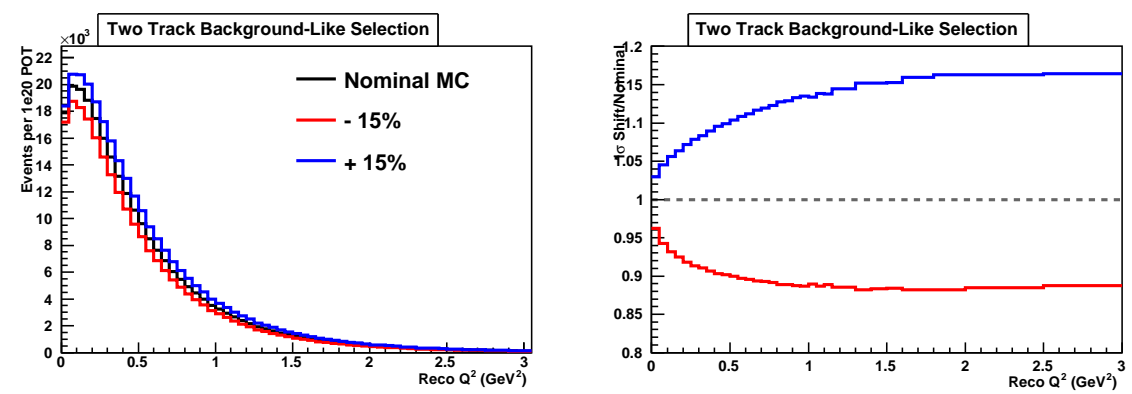

Figure 7.3: Comparison of the nominal MC to reweighted MC with $\pm 15 \%$ changes to the value of $M_{A}^{R E S}$. Only stopping muon events are included in this figure.

- Effective low $\boldsymbol{Q}_{Q E}^{2}$ quasi-elastic suppression $\left(k_{F e r m i}^{Q E}\right)$ : It is expected that the MC will mis-model the data in the very low $Q^{2}$ region due to the poorly understood contribution of nuclear effects to the CCQE cross section. This parameter is a scale factor applied to the value of the Fermi momenta (for either protons or neutrons) used to determine whether a given quasielastic event is Pauli-blocked. The uncertainty on this parameter is hard to determine and likely fairly large (at least 20-30\%) thus an assumed a 30\% error on this parameter for the $\chi^{2}$ penalty term is used. This value for the uncertainty in this parameter is driven by difference in low $Q^{2}$ suppression due 
to different models of the nucleus. In later fits the uncertainty is irrelevant because this parameter is fit unconstrained. In NEUGEN any quasielastic interaction that leaves the initial hadronic state nucleon (pre-INTRANUKE) with a momentum less than the Fermi momentum ( $251 \mathrm{MeV}$ for protons) is considered Pauli-blocked and removed. As such, it is only possible to consider scaling up the value of the Fermi momentum. Figure 7.4 shows the effect of a $+30 \%$ change to this parameter on the 1 track QE-like sample.
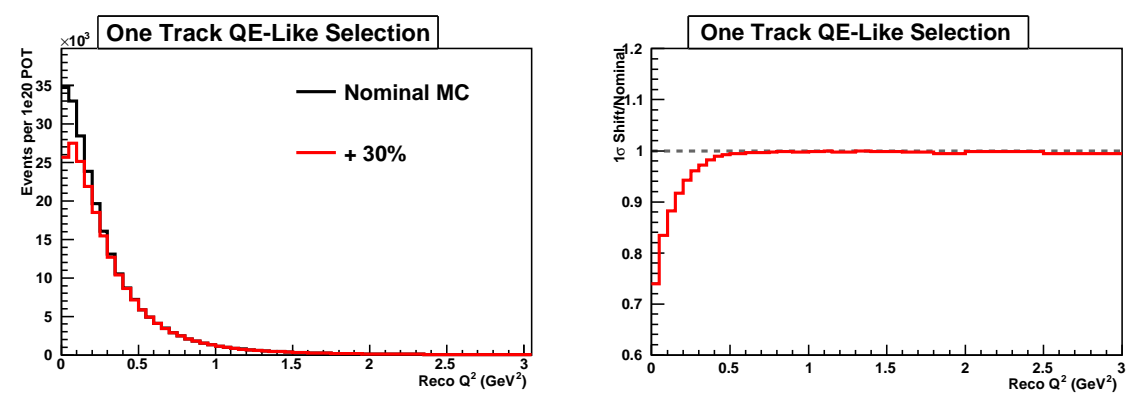

Figure 7.4: Comparison of the nominal MC to MC with a $+30 \%$ scale of the values of the Fermi momentum for protons and neutrons. Only stopping muon events are included in this figure.

- Effective low $\boldsymbol{Q}_{Q E}^{2}$ resonance suppression $\left(k_{F e r m i}^{R E S}\right)$ : This parameter plays a similar role to the effective low $Q_{Q E}^{2}$ suppression parameter but in the modeling of resonant interactions. This parameter suppresses resonance interactions by implementing an ad hoc Pauli-blocking mechanism. In the standard MC, resonant events are not Pauli-blocked, because the intermediate $\Delta(1232)$ does not get Pauli-blocked in the nucleus due to the different quantum numbers of the $\Delta$ and nucleons. The Pauli-blocking is implemented in a semi-physical manner by requiring that the final state nucleon decay products of the $\Delta$ have momentum greater than the Fermi momentum of the nucleons, which is $263 \mathrm{MeV}$ for neutron and $251 \mathrm{MeV}$ for protons. As with the effective low $Q_{Q E}^{2}$ suppression parameter the uncertainty on this parameter is hard to determine and also likely fairly large (at least 20-30\%) and 
so an assumed $30 \%$ uncertainty on this parameter for the $\chi^{2}$ penalty term is used. The uncertainty used for this parameter is also driven by the amount of low $Q^{2}$ suppression seen by different models of the nucleus. However the quoted uncertainty will be irrelevant because this parameter will not be used for the ultimate results of this dissertation. Fig. 7.5 shows the effect of a shift in the effective low $Q_{R E S}^{2}$ to the background-like sample.
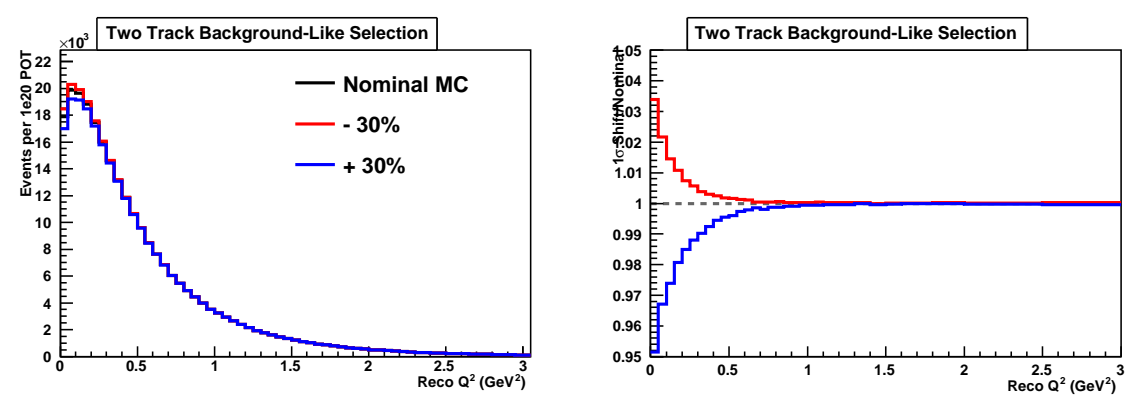

Figure 7.5: Comparison of the nominal MC to MC with a $\pm 30 \%$ scale on the values of the Fermi momentum for protons and neutrons applied to the background like two track selection. Only stopping muon events are included in this figure.

- Hadronic Shower Energy Offset: Because this analysis calculates $Q^{2}$ using the quasi-elastic assumption the only effect this parameter has on the shape of the $Q^{2}$ spectrum is through event migration between event samples or potential migration out of the selected events entirely. With this parameter the analysis allows for the possibility that there is a difference between the shower energy in the MC (presumably perfect) and the data. This parameter allows for the possibility that the shower energy calibration in the data and in the MC is different in the simplest way possible, through an additive constant. A more complicated correction is probably not needed because this parameter only contributes to changes in the shape of the $Q^{2}$ distribution through event migration. 


\subsection{Mock Data Analysis}

There is a total of approximately 725,000 interactions candidates between the three selections that are used in this analysis. With $46 \%$ of the interaction candidates in both the low hadronic energy and the two track background-like subsamples, the remaining $8 \%$ of the interaction candidates are in the two track QElike sub-sample. This means that the statistical error in each bin of $Q^{2}$ is between approximately 1 and 2\%, making this analysis dominated not by statistical uncertainties but systematic uncertainties in the neutrino interaction models. It is useful to finalize all of the the major analysis decisions (how many samples, $Q^{2}$ binning, goodness of fit metric, etc) before proceeding with the analysis on data this will limit the impact of selection biases on the final result. This is done by analyzing a mock data sample first. The mock data sample consists of a subset of the MC that has had the model parameters set to some arbitrary value, while the remaining MC is then used to attempt to recover the mock data model parameters. The analysis procedure is tuned to minimize the uncertainty of the mock data analysis while also minimizing the potential of systematic effects. Once this is done the analysis is repeated on real data, and potentially changed based on what is learned from the differences between data and mock data.

\subsubsection{Mock Data Construction}

The entire MC sample was used to generate a mock data $Q^{2}$ distribution for all three selections. The fit parameters for the mock data distributions were set to the best fit values for the low hadronic energy analysis that has already been done. These $Q^{2}$ distributions were then normalized to the run I pot (1.27e20). These $Q^{2}$ distributions were then used as a PDF to generate new $Q^{2}$ distributions with a number of entries equal to the Poisson fluctuated integral of the original $Q^{2}$ distributions. 


\subsubsection{Mock Data Fit Results}

The fit to the mock data was performed as described in Section 7.1 and generated as described above in Section 7.1. This fit will be compared to the fit to only the low hadronic energy analysis that was presented at the 6th International Workshop on Neutrino-Nucleus Interactions in the Few-GeV Region (NuInt09) [87]. A summary of both of these results are given in Table 7.1, while the $Q^{2}$ distributions for the mock data fits are shown in Figure 7.6.

\begin{tabular}{|l|c|c|}
\hline Parameter & NuInt09 Results & Mock Data Study \\
\hline \hline$M_{A}^{Q E}(\mathrm{GeV})$ & 1.192 & 1.17 \\
$E_{\mu}-$ scale & 0.988 & 0.989 \\
$M_{A}^{R E S}(\mathrm{GeV})$ & 1.112 & 1.100 \\
$k_{F}^{Q E}-$ scale & 1.284 & 1.282 \\
$k_{F}^{R E S}-$ scale & 1.0 (Fixed) & 1.085 \\
\hline$\chi^{2} /$ ndf & 1.023 & 0.536 \\
\hline
\end{tabular}

Table 7.1: Best fit results and for the two fit configurations and reduced $\chi^{2}$ values according to 7.1 for the best fit MC.

The increase in sensitivity from this analysis come from two sources. Breaking of correlations between parameters, and different strengths in different kinematic regions. The three sub-sample analysis reduces the correlations between $M_{A}^{Q E}$ and $k_{F e r m i}^{Q E}$ and also the correlations between $M_{A}^{Q E}$ and $M_{A}^{R E S}$. Referring back to Figure 6.8 the fraction of true QE interactions in the two track QE-like subsample (the blue line from Figure 6.8) shows that there are no true QE interactions within the low $Q^{2}$ region of this sub-sample, thus any low $Q^{2}$ suppression that is seen in this sub-sample can not be coming from true QE interactions. Furthermore by adding a selection that is dominated by the background to the QE-like sub-samples, the behavior of the background (as represented by the $k_{F e r m i}^{R E S}$ and $M_{A}^{R E S}$ parameters) in the QE-like sub-samples can be significantly constrained. These additional sub-samples don't do anything to break the correlations between the $E_{\mu}$-scale however because the other selections much more tightly constrain 
$M_{A}^{Q E}$, the $E_{\mu}$ scale is also constrained due to the $M_{A}^{Q E}-E_{\mu}$ correlations.

\subsubsection{Conclusion}

This mock data study estimates that the three sub-sample fit will reduce by a factor of 2-3 the impact of the fit error to our measurement of the effective $M_{A}^{Q E}$ parameter. And given the impact that other systematics had on the NuInt09 results the expectation would be that those uncertainties would dominate the uncertainty of this analysis.

\subsection{Initial Fit to Data}

Two things were learned from the mock data fit. The mock data fit quantified the expected improvement to the $M_{A}^{Q E}$ measurement from the inclusion of the two, two track selections, while simultaneously demonstrating how the fit parameters are correlated when the fit is performed with multiple sub-samples. Because the two track selections do a good job of breaking the correlations between, $M_{A}^{Q E}$ and $k_{F e r m i}$, and $M_{A}^{Q E}$ and $M_{A}^{R e s}$ it becomes possible to fit these parameters unconstrained by a penalty term. Thus the first fit to data is performed with all of the parameters from the mock data fit as free parameters with the exception of the muon energy scale.

\section{Fit Results}

The initial fit to data was performed as described in Section 7.1 with the modifications outlined in Section 7.3. The resulting $Q_{Q E}^{2}$ distributions are shown in Figure 7.8. The best fit values are shown in Table 7.2.

The best fit values for this fit configuration are very different from the best fit values given by the MINOS NuInt 2009 analysis [87]. Furthermore the $\chi^{2}$ for this fit configuration is $\sim 18 \sigma$ from the expected reduced $\chi^{2}$ for a fit with 49 degrees 

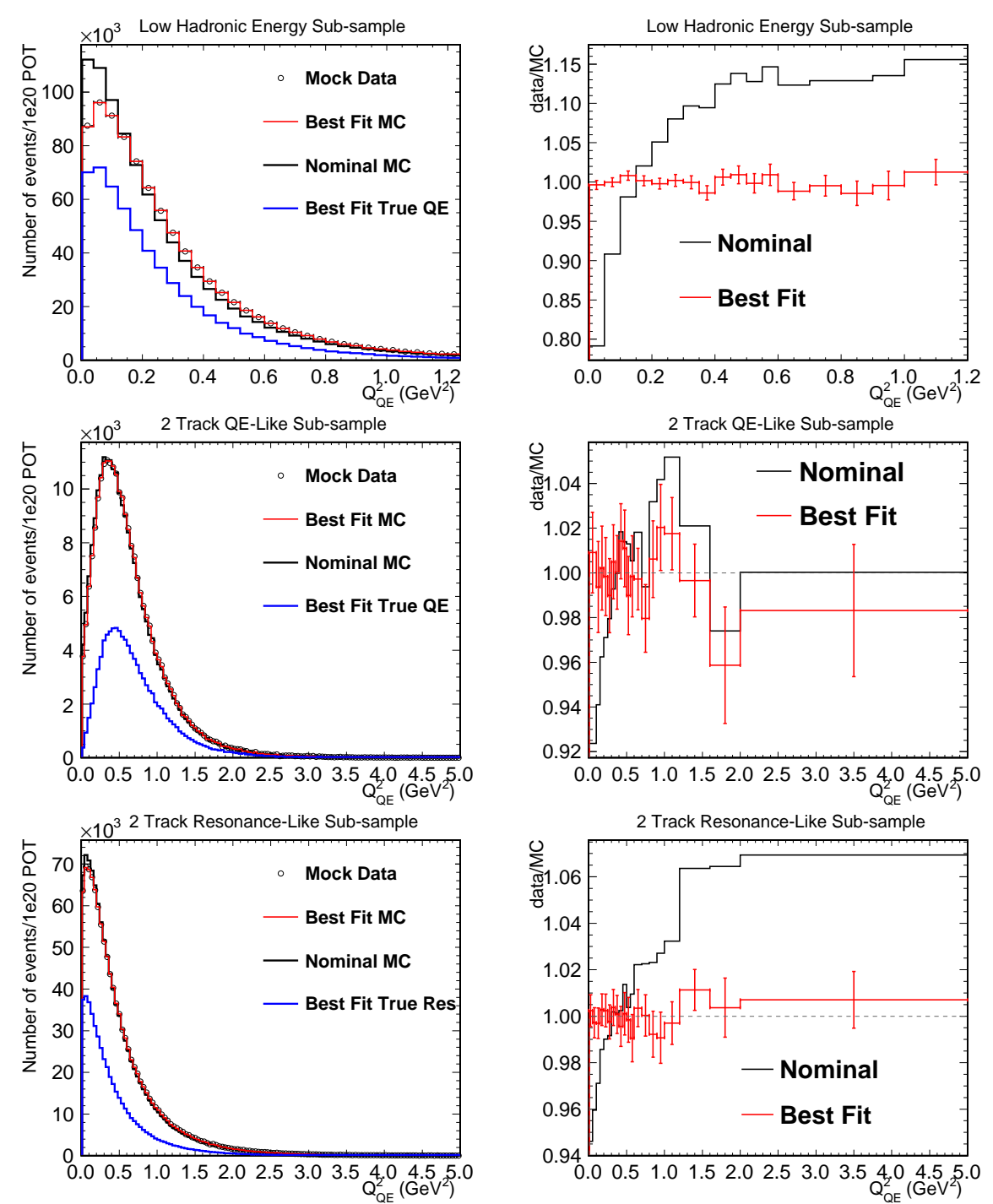

Figure 7.6: Starting from the top and moving down are the $Q^{2}$ distributions for the low hadronic shower energy sub-sample, the 2 track QE-like sub-sample, and the 2 track background-like sub-sample. On the left are the actual $Q^{2}$ distributions using a binning that is much finer than the binning actually used in the fits. On the right are data/MC plots using the actual fit binning. The blue histograms show the dominant topology for each of the three selections. 

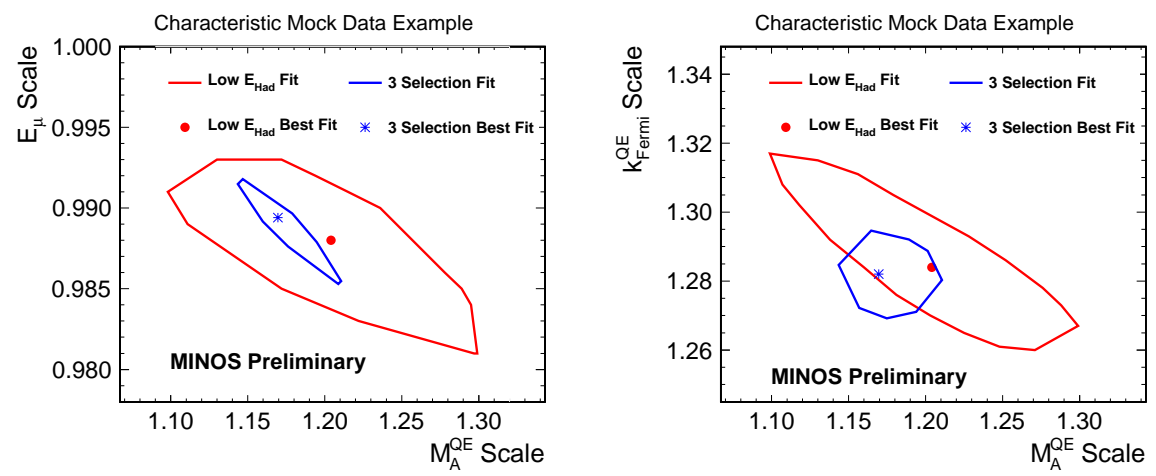

Figure 7.7: The 1- $\sigma$ (defined as $\Delta \chi^{2}=1$ ) contours for the 1 track NuInt09 analysis and this mock data analysis. The contour for $M_{A}^{Q E}$ and the $E_{\mu}-$ scale is shown on the left. The contour for $M_{A}^{Q E}$ and $k_{\text {Fermi }}^{Q E}-$ scale is shown on the right. The one track NuInt09 result contour is shown in red. The contours from this mock data analysis are shown in blue. The three selection analysis significantly improves the size of the allowed parameter space. In addition the additional selections break the correlation between $M_{A}^{Q E}$ and the $k_{F e r m i}^{Q E}$-scale.

\begin{tabular}{|l|c|}
\hline Parameter & Initial fit to data \\
\hline \hline$M_{A}^{Q E}(\mathrm{GeV})$ & $1.000 \pm 0.033$ \\
$E_{\mathrm{V}}-$ scale & $0.992 \pm 0.002$ \\
$M_{A}^{R E S}(\mathrm{GeV})$ & $1.078 \pm 0.024$ \\
$k_{F}^{Q E}-$ scale & $2.87 \pm 0.04$ \\
$k_{F}^{R E S}-$ scale & $1.14 \pm 0.01$ \\
\hline$\chi^{2} /$ ndf & 2.65 \\
\hline
\end{tabular}

Table 7.2: Best fit values for the fit to data. The errors shown are the MINUIT returned HESSE errors.

of freedom. Thus this fit is a poor description of the data and the best fit values for the fit parameters are meaningless.

In this fit, the parameter $k_{F e r m i}^{R e s}$ has a very different value from the assumed nominal value of 1 . The mock data fit had a assumed one sigma error of $30 \%$, the 

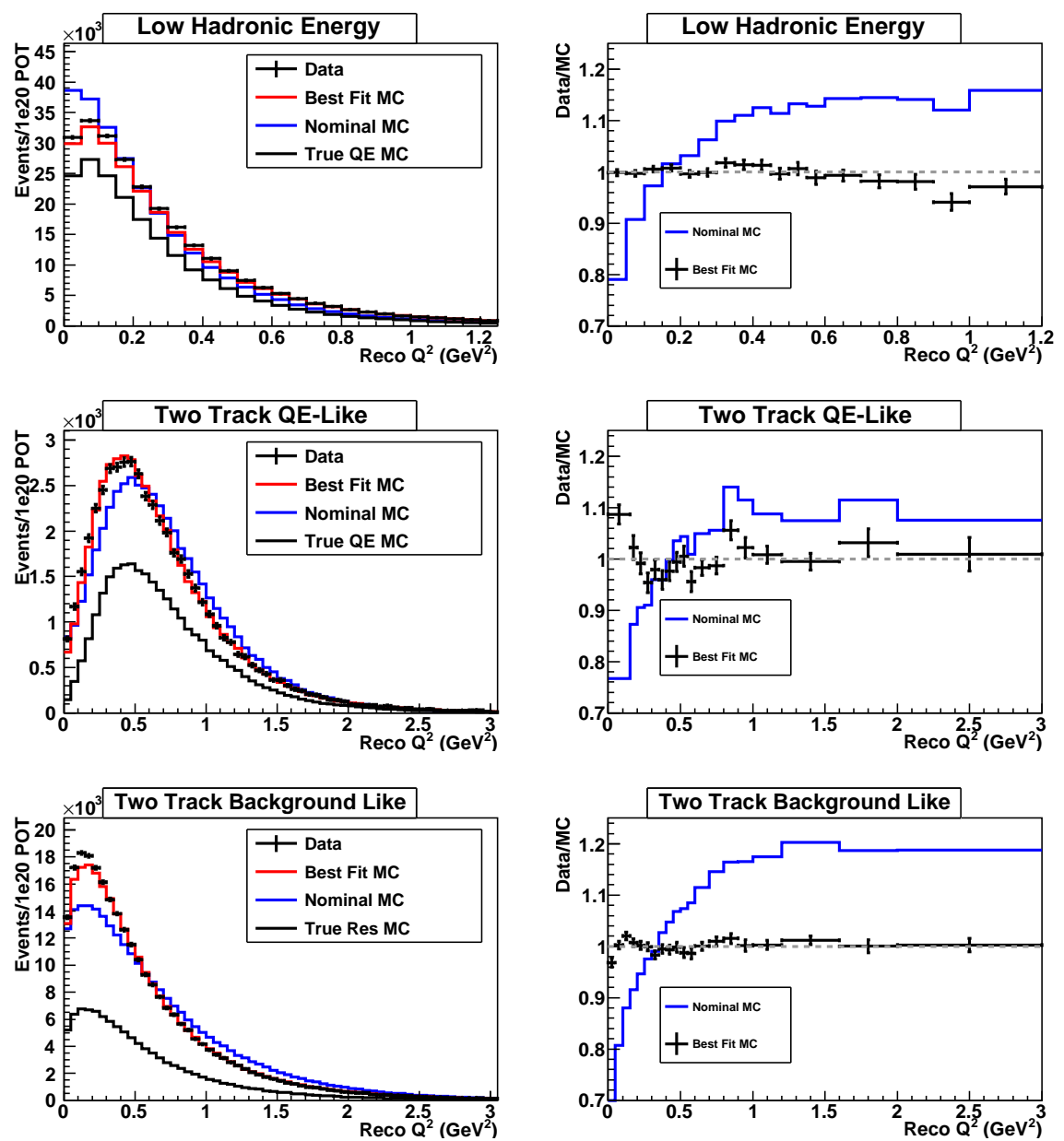

Figure 7.8: Best fit $Q_{Q E}^{2}$ distributions for the fit to data that matches the mock data procedure. Top: One track QE-like selection. Middle: Two track QE-like selection. Bottom: Two track resonance-like selection. Left: Finely binned $Q_{Q E}^{2}$ distribution. Red: Best fit $Q_{Q E}^{2}$ distribution. Blue: Nominal $Q_{Q E}^{2}$ distribution. Black: Best fit $Q_{Q E}^{2}$ distribution of the dominant interaction type of each selection. Right: Data/MC ratio binned using the same binning as the fit. 
best fit value here is $6 \sigma$ away from the mock data fit assumed nominal value. This $k_{F e r m i}^{R e s}$ is suspiciously large, Figure 7.9 compares the nominal resonance MC to the resonance $\mathrm{MC}$ with $k_{F e r m i}^{Q E}$ equal to 2.87. Figure 7.9 demonstrates that even as high as a $Q_{Q E}^{2}$ value of 1 , this suppression scheme removes $10 \%$ of the resonances events. The larger than expected suppression of resonance events in the moderate $Q^{2}$ region produced by $k_{F e r m i}^{R e s}$ equal to 2.87 disagrees with the ad-hoc suppression function from R. Gran's and N. Graf's work in [88].
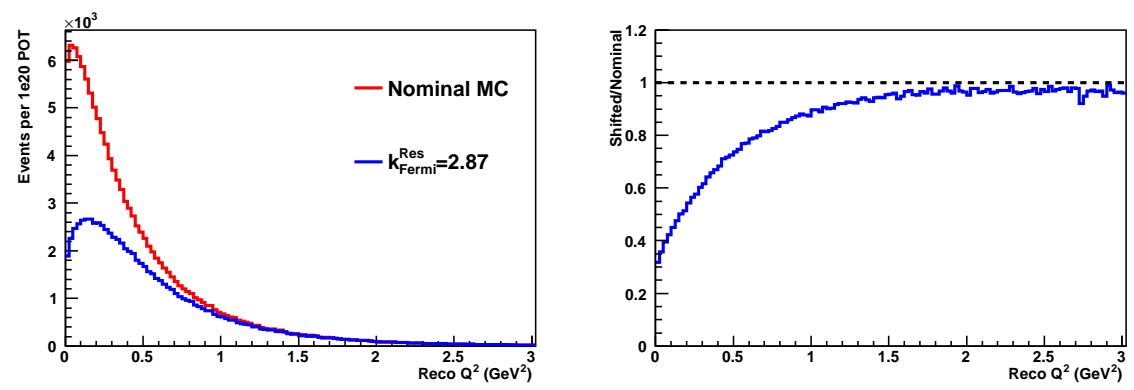

Figure 7.9: Left: true resonance $Q_{Q E}^{2}$ distributions. Blue: distribution for $k_{F e r m i}^{R e s}$ equal to 2.87. Red: nominal true resonance $Q_{Q E}^{2}$ distribution. Right: the $k_{F e r m i}^{R e s}$ equal to 2.87 distribution divided by the nominal distribution.

In [88] the authors extract a suppression function for the $Q^{2}$ distribution of the resonances using two separate resonance enhanced sub-samples. These event sub-samples are defined using a simple cut on $\mathrm{W}$, the hadronic invariant mass. The two selections they define are: The Delta sub-sample defined as $\mathrm{W}$ between 0 and $1.3 \mathrm{GeV}$ and the Transition sub-sample defined as $\mathrm{W}$ between 1.3 and $2.0 \mathrm{GeV}$. The Delta sub-sample is dominated by resonances and QE, while the Transition is dominated by resonances and DIS. Using these two sub-samples the authors implemented a smoothly shaped suppression function with a shape motivated by theoretical [89][90] and experimental [34] work on the $Q^{2}$ shape of resonance interactions. Using this shape as a starting point the authors then tuned the shape by reducing the residuals in the Delta and Transition sub-samples to get the final 
suppression shape shown in Figure 7.10 which is compared to the suppression that results from a $k_{F e r m i}^{R e s}$ value of 2.87. By design the suppression function from [88] turns off for $Q^{2}$ values greater than $0.6 \mathrm{GeV}^{2}$, while the $k_{F e r m i}^{\text {Res }}$ suppression in the same region is still about $70 \%$. The natural conclusion from the poor $\chi^{2}$ calculated from this fit and the poor agreement between the $k_{\text {Fermi }}^{\text {Res }}$ suppression and the suppression from [88] is that the $k_{F e r m i}^{R e s}$ suppression is unphysical.
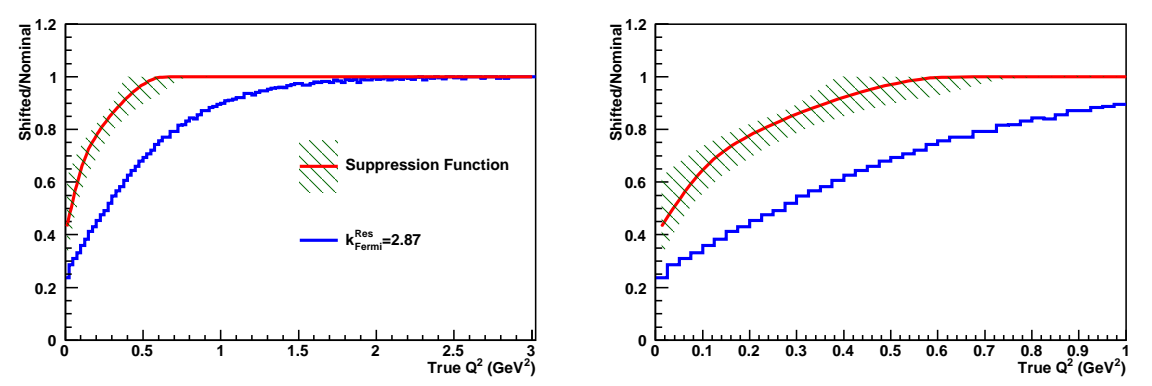

Figure 7.10: Red: suppression function from [88] with error band that includes both statistical and systematic uncertainty. Blue: suppression function from a $k_{F e r m i}^{R e s}$ value of 2.87 .

\subsection{Fit Using The Resonance Suppression Function}

The natural progression from the initial fit to the data is a fit that incorporates the resonance suppression function from [88] instead of the $k_{F e r m i}^{R e s}$ based suppression. This fit will use a more realistic suppression function, however the low $Q^{2}$ suppression cannot be further tuned to match the data, consequently there is no longer a parameter that describes the behavior of the low $Q^{2}$ resonances interactions. The resulting $Q^{2}$ distribution from this fit are shown in Figure 7.11. The best fit values for the parameters are shown in Table 7.3. This fit has 50 degrees of freedom instead of 49 because there is one less fit parameter.

This fit with the low $Q^{2}$ resonances suppression function is slightly worse than 

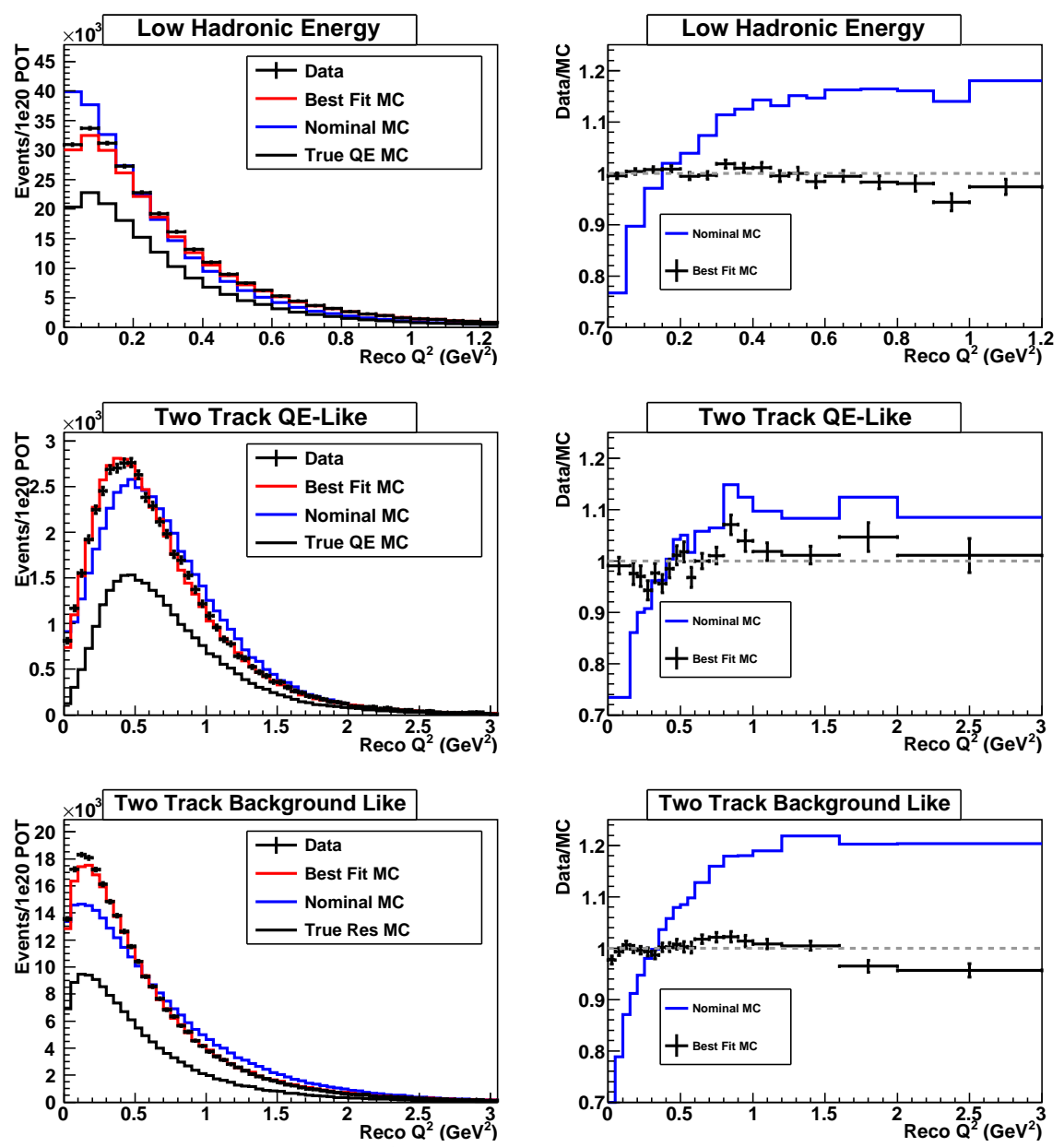

Figure 7.11: Best fit $Q_{Q E}^{2}$ distributions for the fit to data that uses the low $Q^{2}$ suppression function from [88]. Top: One track QE-like selection. Middle: Two track QE-like selection. Bottom: Two track resonance-like selection. Left: Finely binned $Q_{Q E}^{2}$ distribution. Red: Best fit $Q_{Q E}^{2}$ distribution. Blue: Nominal $Q_{Q E}^{2}$ distribution. Black: Best fit $Q_{Q E}^{2}$ distribution of the dominant interaction type of each selection. Right: Data/MC ratio binned using the same binning as the fit. 


\begin{tabular}{|l|c|}
\hline Parameter & Fit With Suppression Function \\
\hline \hline$M_{A}^{Q E}(\mathrm{GeV})$ & $1.274 \pm 0.032$ \\
$E_{\mathrm{v}}-\mathrm{scale}$ & $0.978 \pm 0.003$ \\
$M_{A}^{R E S}(\mathrm{GeV})$ & $1.502 \pm 0.019$ \\
$k_{F}^{Q E}-$ scale & $1.107 \pm 0.007$ \\
\hline$\chi^{2} / \mathrm{ndf}$ & 2.87 \\
\hline
\end{tabular}

Table 7.3: Best fit values for the fit to data using the suppression function from [88]. The errors shown are the MINUIT returned HESSE errors.

the fit in section 7.3, which can be attributed to the lack of a parameter that tunes the low $Q^{2}$ suppression of the resonances. The largest source of disagreement between the data and the MC is in the middle and bottom plots in Figure 7.11 in the less than $1 \mathrm{GeV}^{2}$ region. It would be useful to look at the contribution to the $\chi^{2}$ from each individual sub-sample. The contribution to the $\chi^{2}$ from each individual sub-sample helps determine which sub-samples are being poorly modeled by the parameters in the $\mathrm{MC}$ and what sub-samples are being modeled well. Determining the $\chi^{2}$ contribution from the individual selections can be done by looking at confidence contours for each individual fit and for the combined fit. These contours are shown in Figure 7.12.

From Figure 7.12 the strengths and weakness of the individual sub-samples relative to the combined fit really become apparent. The background-like subsample (depicted in violet) prefers significantly different values for the parameters compared to the other individual sub-samples, except for the $M_{A}^{\text {Res }}$ parameter the background-like sub-sample does not overlap with any of the other individual subsample. Section 6.4.1 discussed significant differences between the MC and the data, differences that are in the resonance dominated sideband to the two track QElike sub-sample. All of the interaction candidates selected in the two track QElike sideband are also in the two track background-like selection. The two track resonance selection adds a some interaction candidates due to the loosening of the 

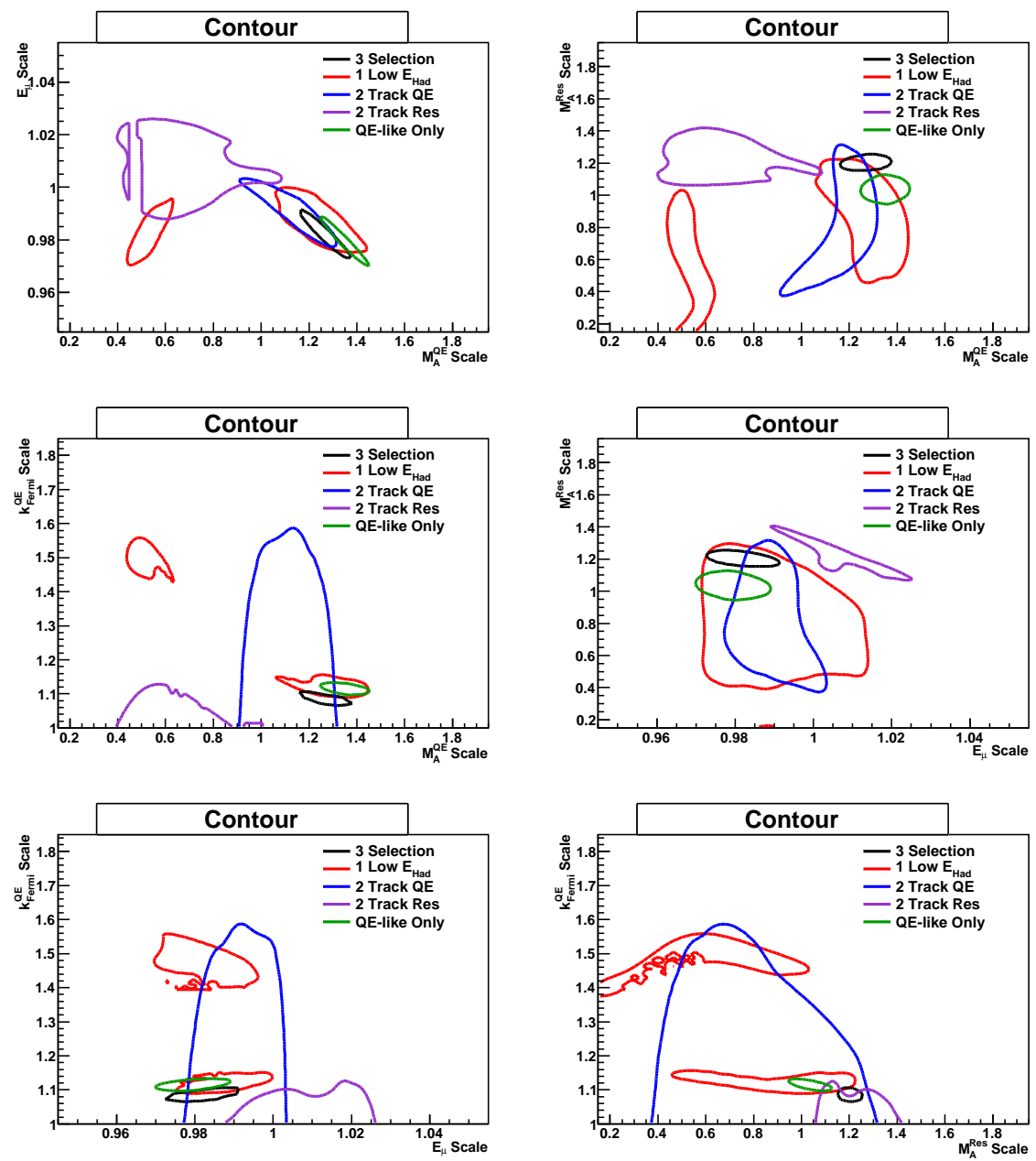

Figure 7.12: One sigma contours for all the different pairs of fit parameters. For each plot the parameters not shown are marginalized. Black: three sample combined fit. Red: one track QE only fit. Blue: two track QE only fit. Violet: two track resonance only fit. Green: one and two track QE combined fit. Starting with the top left: $M_{A}^{Q E}$ versus $E_{\mu}$-scale, $M_{A}^{Q E}$ versus $M_{A}^{R e s}, M_{A}^{Q E}$ versus $k_{F e r m i}^{Q E}, E_{\mu}$-scale versus $M_{A}^{\text {Res }}, E_{\mu}$-scale versus $k_{\text {Fermi }}^{Q E}, M_{A}^{\text {Res }}$ versus $k_{\text {Fermi }}^{Q E}$. 
unused hits selection criteria. Thus any differences between data and MC in the two track QE sideband are expected to show up in the two track background-like sub-sample. The parameters used to describe the three sub-sample in this analysis are sufficient to describe each individual selection, however they are not sufficient to describe all three sub-samples simultaneously. This supports the hypothesis that there is one or more unsimulated types of interactions in the data.

There is enough evidence that there are one or more types of unsimulated interactions getting classified as background interactions to warrant the inclusion of some kind of background correction function within this analysis. However there are clearly correlations between the parameters that control the shape of the background and the parameters that control the shape of the signal. The ideal solution is what has already been tried, fit everything simultaneously, however that no longer seems possible. It appears that a whole new fit procedure is needed.

\subsection{New Fit Procedure}

Unsurprisingly the tension that exists between the two track background-like subsample and the two QE-like sub-samples is primarily in the parameter that controls the shape of the background. Individually $M_{A}^{R e s}$ is sufficient to describe the shape of the background. The $\chi^{2}$ for all the individual fits are reasonable given the number of degrees of freedom, $M_{A}^{R E S}$ is only insufficient when trying to use it to describe the shape of the resonances in all three sub-samples simultaneously. Because $M_{A}^{\text {Res }}$ is sufficient to describe the shape of the resonances in each selection individually, but not sufficient to describe all three selections simultaneously, it is necessary to develop a fit procedure that extracts the data/MC differences in the resonances events that aren't being described using the $M_{A}^{R e s}$ parameter.

\subsubsection{Fit Steps}

A fit procedure that meets all of the preceding requirements can be constructed using the following steps: 
1. Fit: fit only the QE-like selections.

2. Characterize the QE: feed the best fit parameters from the simultaneous fit of both QE-like selections into the two track resonance selection.

3. Event Subtraction: subtract the predicted true QE events from the data in the two track resonance selection.

4. Fit two track resonance: fit the two track resonance selection (with no true QE events) using the $M_{A}^{\text {Res }}$ parameter as the only free parameter, every other parameter is fixed.

5. Background Correction: use the data/MC spectrum from the two track resonance selection, parameterized using a cubic polynomial, as a correction to the background of the QE-like selections.

6. Repeat Fit: perform this procedure again until the background correction function agrees with the background correction function of the previous iteration.

The disadvantage in this fit procedure is the best fit value of the $M_{A}^{\text {Res }}$ parameter is essentially meaningless. In effect there are two different best fit values for $M_{A}^{\text {Res }}$ : One from the simultaneous fit of the QE-like sub-samples, and one from the from the fit of the two track background-like sub-sample. The $M_{A}^{\text {Res }}$ parameter is being used instead to describe (along with the background correction function) the shape of what appears to be the resonances plus some other type of interactions that are not simulated in the MINOS MC.

Even though the value for the $M_{A}^{\text {Res }}$ parameter is essentially meaningless, the background correction function provides more confidence in the measurement for $M_{A}^{Q E}$. By using the background correction function plus the shape from $M_{A}^{\text {Res }}$, it is possible to account for correlations between the background shape and $M_{A}^{Q E}$, while also accounting for large differences in the background between the data and model. 


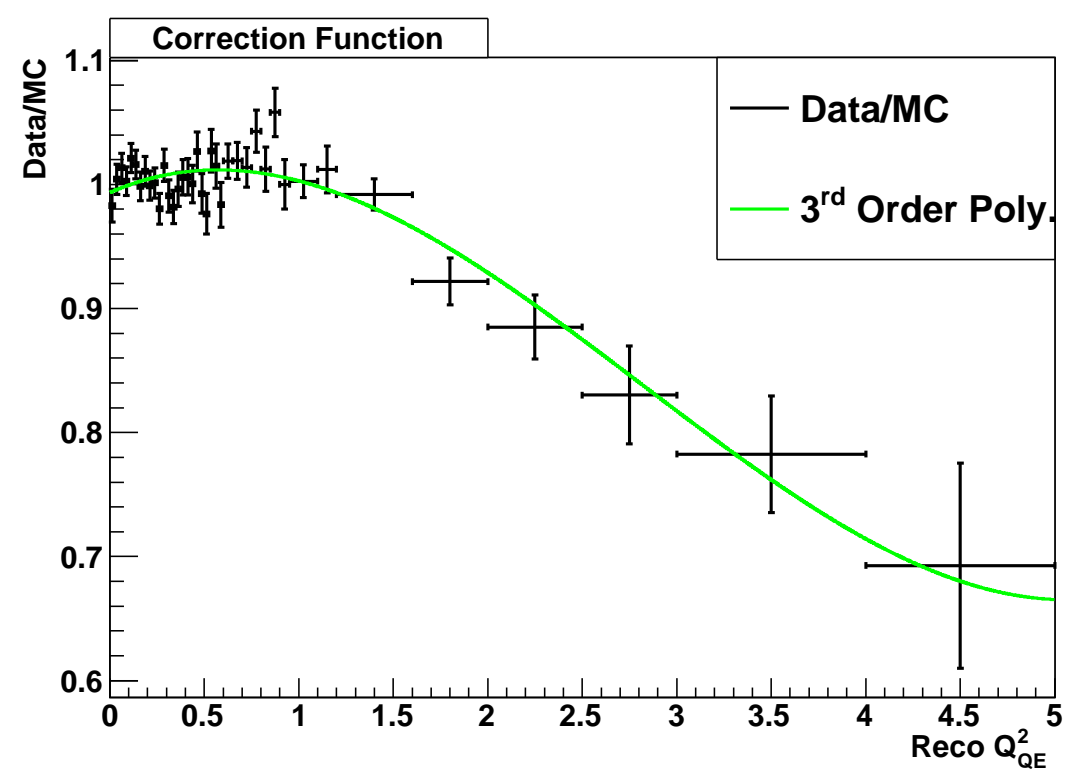

Figure 7.13: The background correction function for the primary fit using the procedure outlined in Section 7.5.1. The correction function is calculated from by fitting the ratio of the data to the $\mathrm{MC}$ from the two track background like sub-sample. This data is fit to a third order polynomial which is the green line shown in the Figure.

\subsubsection{Hadronic Shower Energy Offset}

The hadronic shower energy offset fit parameter changes the $Q^{2}$ spectrum by causing interactions candidates to migrate between the three sub-samples. Because this new fit procedure doesn't fit the signal and the background simultaneously it is important that the any large scale migrations are accounted for in the first fit iteration. The hadronic shower energy cut is the largest source of the these large scale migration of interaction candidates, and the hadronic shower energy offset was one of the largest sources of systematic error in the first MINOS CCQE analysis [91]. This analysis may be more sensitive to the hadronic energy offset because interaction candidates don't just migrate into and out of the sub-samples by can also migrate between event samples. Because the hadronic energy offset 
had a large effect on the first MINOS CCQE analysis and this analysis may be more sensitive to the hadronic energy offset, the hadronic energy offset is fit as an additional parameter in the new fit procedure.

\subsubsection{Fit Results}

The fit was performed as outlined above. It was found that the background correction function from the third iteration agreed with the background correction function from the second iteration, thus a third fit of the QE-like sub-samples was not necessary. The best fit results from both the first and second fit iterations are shown in Table 7.4. The $Q_{Q E}^{2}$. distributions for the $\mathrm{QE}$-like sub-samples are shown in Figure 7.14 and the background correction function is shown in Figure 7.13.

\begin{tabular}{|l|c|c|}
\hline Parameter & $1^{\text {st }}$ Iteration & $2^{\text {st }}$ Iteration \\
\hline \hline$M_{A}^{Q E}(\mathrm{GeV})$ & $1.312 \pm 0.033$ & $1.315 \pm 0.034$ \\
$E_{\mu}$-scale & $0.985 \pm 0.003$ & $0.986 \pm 0.003$ \\
$E_{\text {had }}$-offset & $0.0381 \pm 0.035$ & $0.0381 \pm 0.035$ \\
$M_{A}^{R E S}(\mathrm{GeV})$ & $1.283 \pm 0.057$ & $1.256 \pm 0.057$ \\
$k_{\text {Fermi }}^{Q \text {-scale }}$ & $1.124 \pm 0.009$ & $1.120 \pm 0.009$ \\
\hline$\chi^{2} /$ ndf & 1.201 & 1.091 \\
\hline
\end{tabular}

Table 7.4: Best fit values for the baseline first and second fit iteration using the procedure outlined in Section 7.5.1. No further iterations were necessary because there was very little change in the background correction function. The errors shown are the HESSE errors from the MINUIT software package.

There is very little change in the $M_{A}^{Q E}$ parameter from the first iteration to the second iteration. While there is a larger (though not statistically significant) change in the $M_{A}^{R E S}$ parameter. This gives confidence that the $M_{A}^{Q E}$ parameter is modeling the behavior of the true quasi-elastic interactions within the sub-samples and not instead accounting for potential model errors within the resonance model. Furthermore the $\chi^{2}$ is reduced from the first to the second iteration, this indicates 
that the background correction function that is defined by the background like sub-sample is increasing the goodness of fit in the QE-like sub-sample.
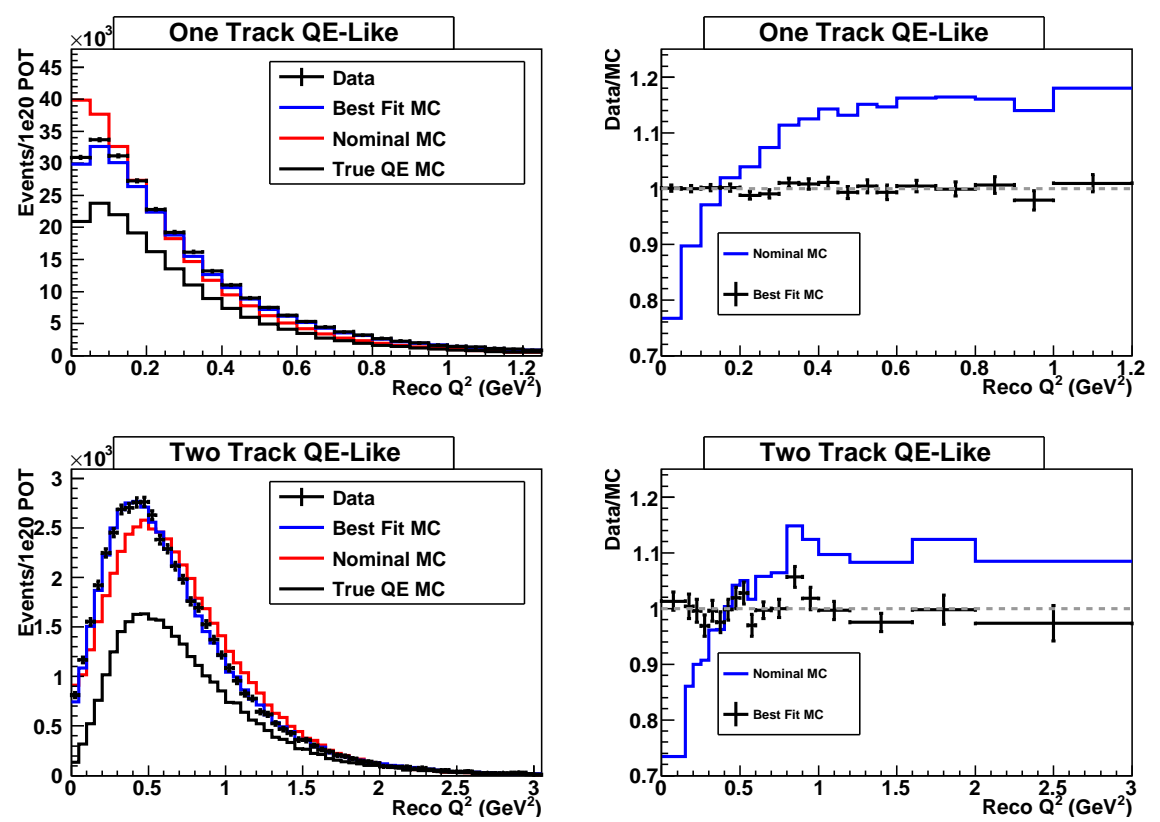

Figure 7.14: Best fit $Q_{Q E}^{2}$ distributions for the fit to data using the procedure outlined in Section 7.5.1. Top: One track QE-like selection. Bottom: Two track QE-like selection. Red: Best fit $Q_{Q E}^{2}$ distribution. Blue: Nominal $Q_{Q E}^{2}$ distribution. Black: Best fit $Q_{Q E}^{2}$ distribution of the true $\mathrm{QE}$ composition of the sub-sample. Right: Data/MC ratio binned using the same binning as the fit.

\subsubsection{Systematic Error Contribution}

Many other factors contribute to the systematic uncertainty in the quasi-elastic axial-vector mass, beyond those that are included directly in the fit. These additional factors (outlined below) are calculated by re-performing the fit procedure outline in Section 7.5.1, only the final fit results (from Table 7.4 are used as the 
initial seed to the fit. The contribution from these various categories are shown in Table 7.5.

\section{Sub-sample Selection Criteria}

This set of systematic shifts consist of changes to the selection criteria that is used to define the various sub-samples used in this analysis. Changing any of these parameters causes interaction candidates to migrate between the defined sub-samples, or potentially out of one of the defined sub-samples and into a the sub-sample of interaction candidates that passed the preselection cuts but were not sorted into one of the defined sub-samples. The quadratic sum of all of the systematic shift is ${ }_{-0.183}^{+0.100}$.
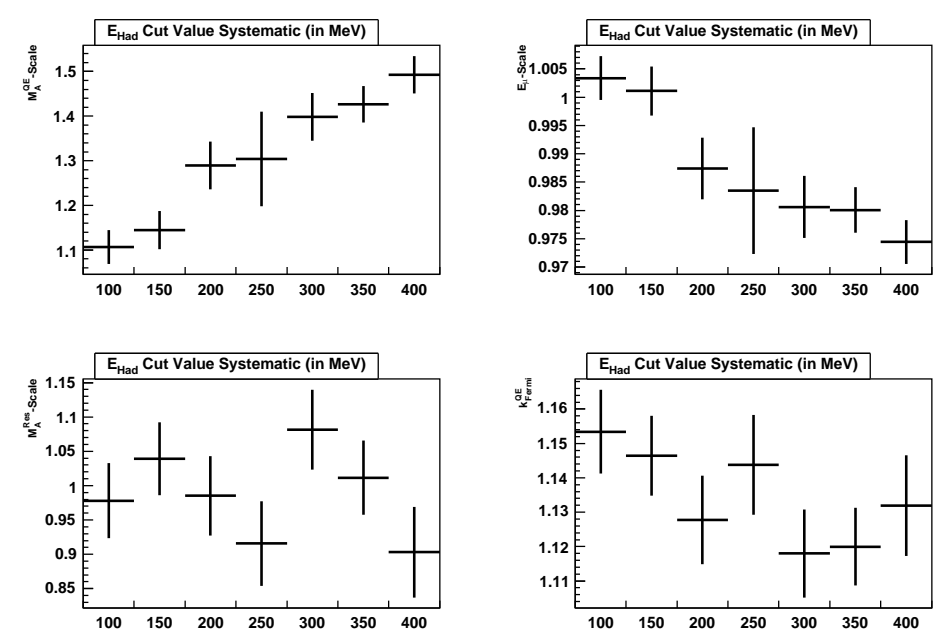

Figure 7.15: Best fit values (along with the HESSE) errors for different definitions of the low hadronic energy sub-sample.

- Low Hadronic Energy Selection Criteria: the low hadronic energy subsample is defined as: all of the preselected interaction candidate with reconstructed hadronic shower energy less than $250 \mathrm{MeV}$. Shown in Figure 7.15 

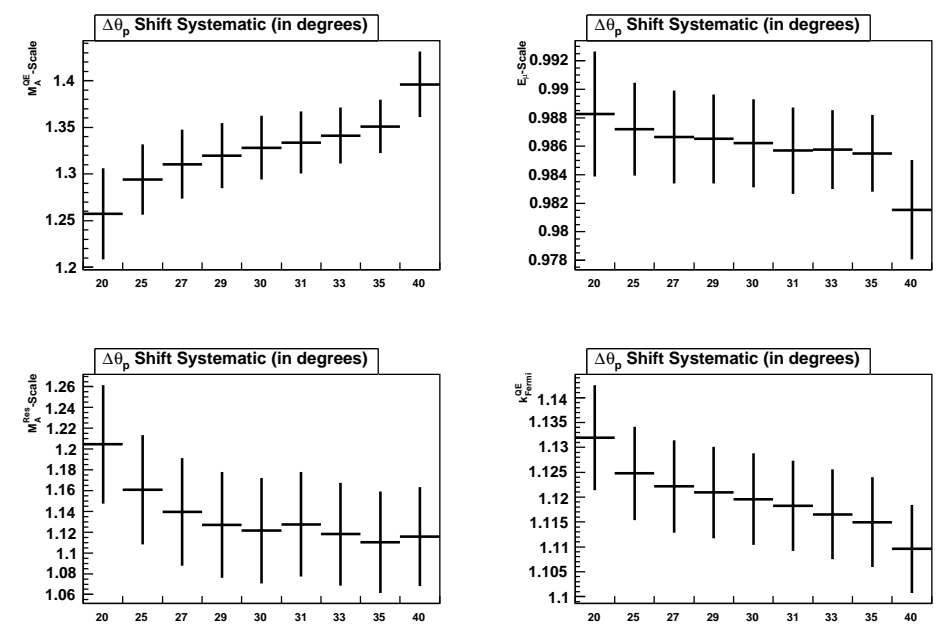

Figure 7.16: Best fit values (along with the HESSE errors) for different definitions of the $\Delta \theta_{p}$ dividing line between the two track QE-like and two track background like sub-samples.

are distributions of possible alternative definitions to the low hadronic energy selection criteria. A previous study of kinematic resolution in MINOS showed that the appropriate (though conservative) value to express a ' $1 \sigma$ ' change in the definition of the low hadronic energy sub-sampel is $\pm 100 \mathrm{MeV}$. This systematic one of the largest single contributions to the uncertainty on the quasi-elastic axial-vector mass.

- $\Delta \theta_{p}$ Selection: this selection variable in part defines the border between the two track QE-like sub-sample and the two track background like subsample. This definition was chosen to select the most low $\Delta \theta_{p}$ 'bump' of true $\mathrm{QE}$ interactions shown in Figure 7.16, while also optimizing the purity of the two track QE like subsample. This somewhat arbitrary choice of selection criteria is allowed to vary along the downward slope of this true QE bump. $\pm 5^{\circ}$ defines the ' $1 \sigma$ ' change in this systematic. 

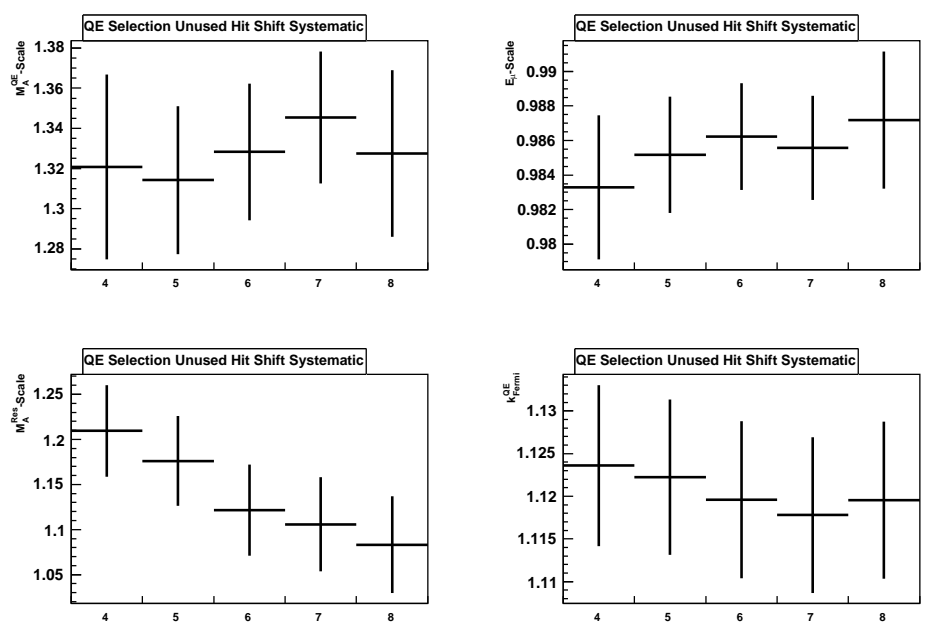

Figure 7.17: Best fit values (along with the HESSE errors) for different definitions of the unused shower strip hit dividing line between the two track QE-like and two track background like sub-samples.

- Unused Hit Selection: the unused hit variable is the other variable that is used to define the separation between two track QE-like sub-sample from the two track background like sub-sample, the assumed ' $1 \sigma$ ' error in this value is \pm 2 strip hits. A different larger value of this variable defines the separation between the two track background like sub-sample from interaction candidates that are not used in this analysis, the assumed ' $1 \sigma$ ' error for this other selection criteria is \pm 4 strip hits. This variable shows very little difference between data and MC, thus it is expected that there will be little change in the best fit values as these selection criteria are changed, and in fact this is what happens for both of these selection criteria. The parameter best fit shifts for these two selection criteria is shown in Figure 7.17 and Figure 7.18. 

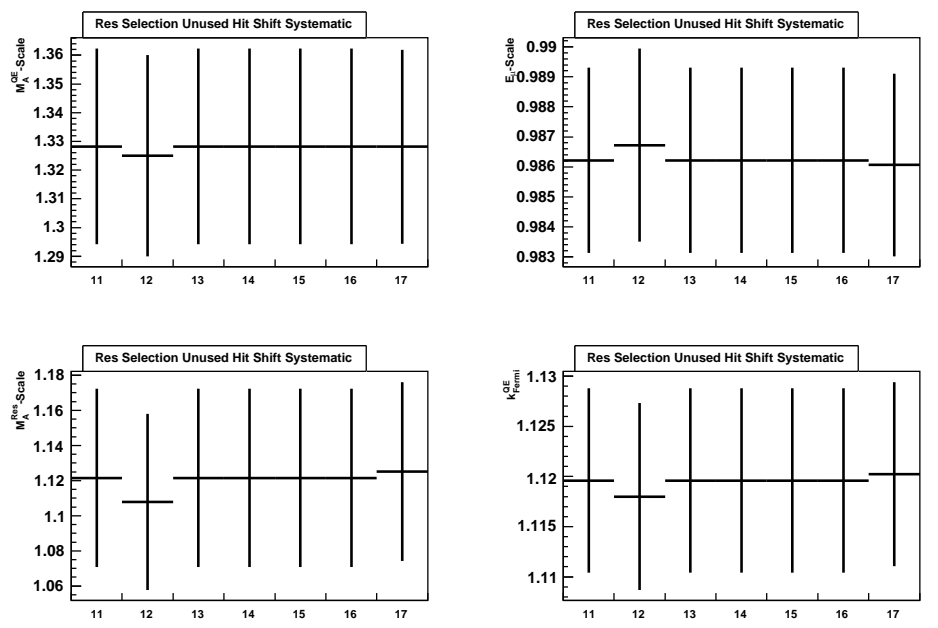

Figure 7.18: Best fit values (along with the HESSE errors) for different definitions of the unused shower strip hit dividing line between the two track background like and the sub-sample of interaction candidates that are not used in this analysis.

\section{Beam Flux Uncertainty}

The beam flux is applied a little differently in this analysis then in the MINOS oscillation analysis for which the procedure was originally developed. Hadron production weights are applied to the primary fit (Table 7.4) then systematic shifts are considered for all of the beam optics nuance parameters (excepting the POT error which is only a normalization error and irrelevant for a shape only fit). While parameters very similar to the detector parameters are already applied within the primary fit. Furthermore additional systematics are considered where the hadron production parameters are reset to there nominal values. An additional shift of $\pm 5 \mathrm{~cm}$ shift in the $z$ position of the target is also considered. This final systematic shift does not express an uncertainty of $\pm 5 \mathrm{~cm}$ to the actually target position but is instead a stand in for some other unknown effect that could be causing the peak of the entire neutrino energy distribution to move. The quadratic sum of the beam 

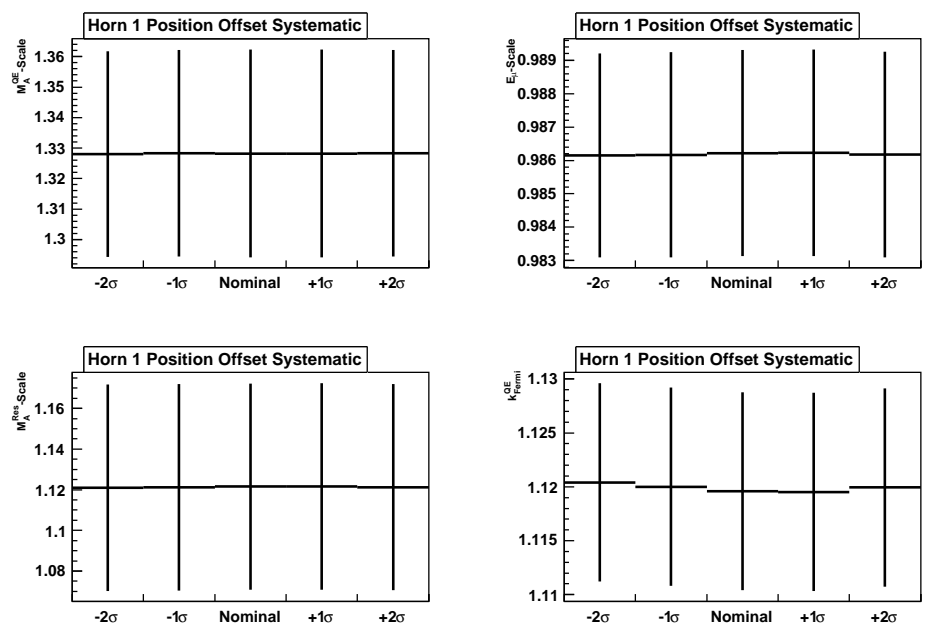

Figure 7.19: Best fit values (along with the HESSE errors) for the horn position systematic.
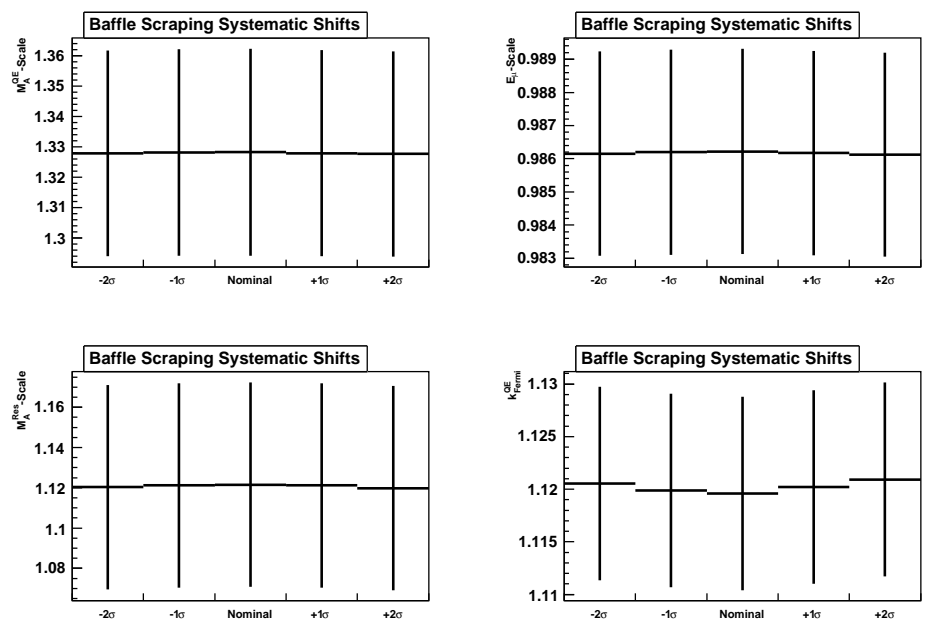

Figure 7.20: Best fit values (along with the HESSE errors) for the baffle scrapping systematic. 

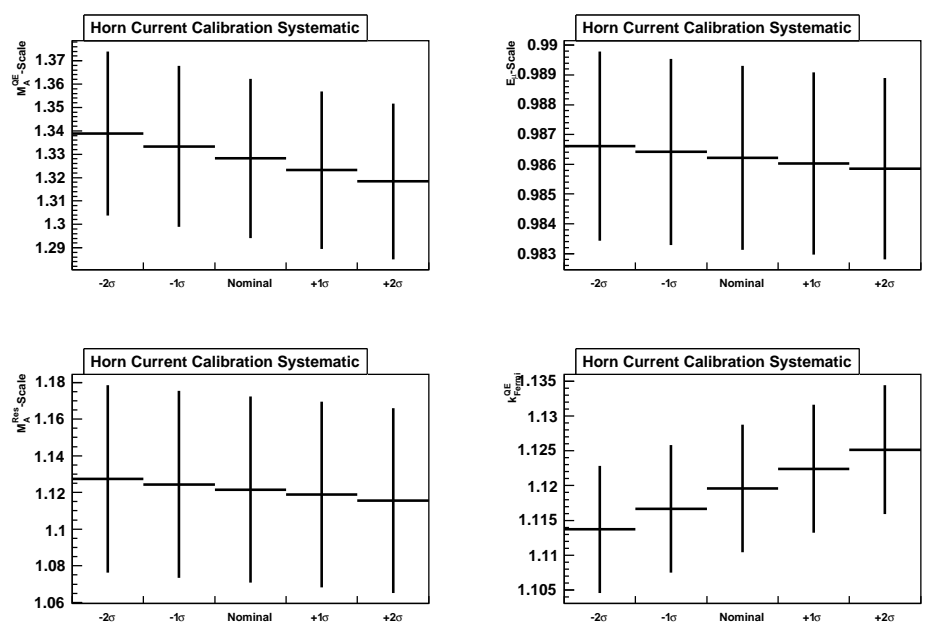

Figure 7.21: Best fit values (along with the HESSE errors) for the horn current calibration systematic.
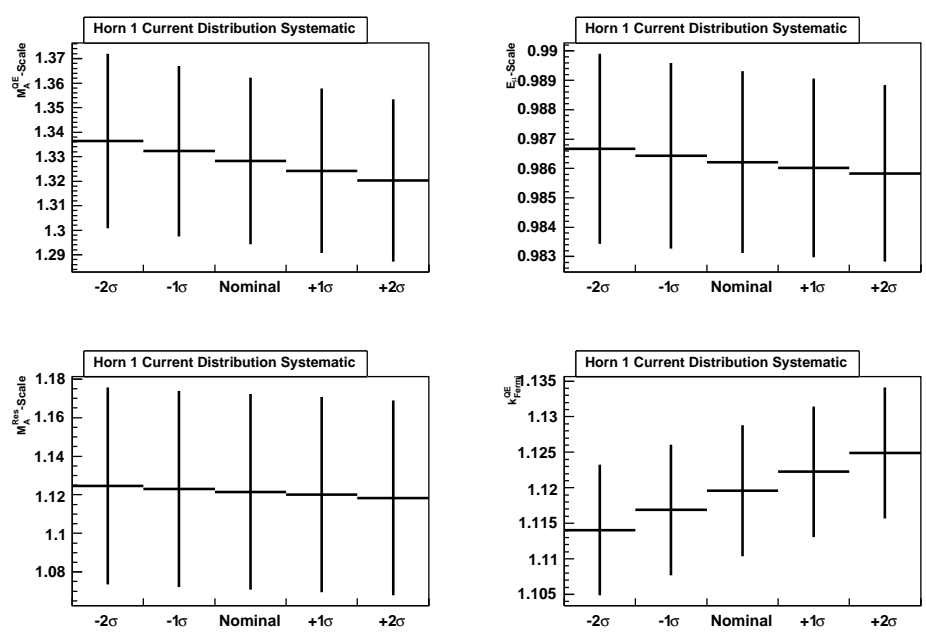

Figure 7.22: Best fit values (along with the HESSE errors) for the horn current distribution systematic. 
flux uncertainty contribution to the quasi-elastic axial-vector mass is ${ }_{-0.019}^{+0.020}$.
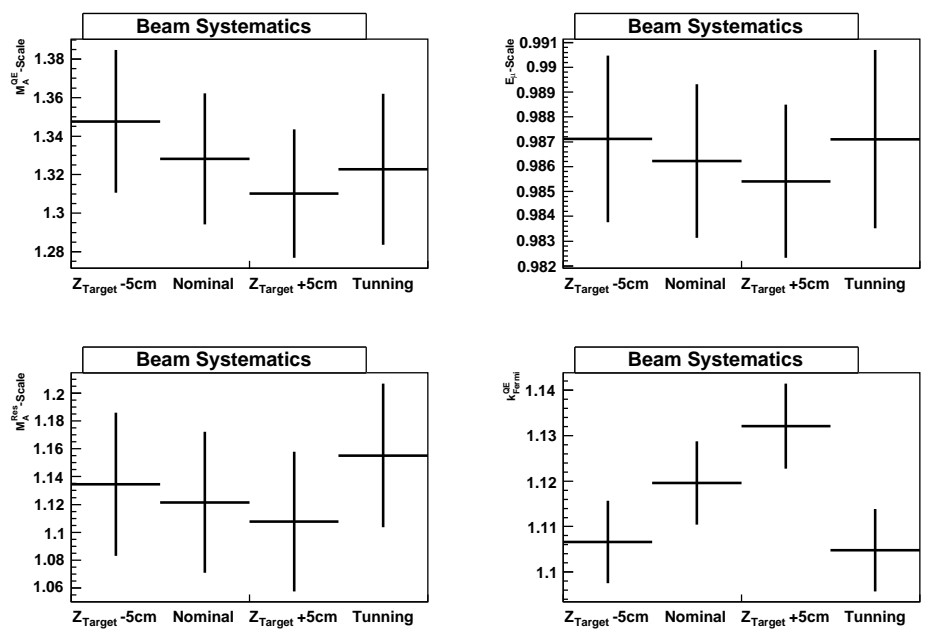

Figure 7.23: Best fit values (along with the HESSE errors) for the target $z$ position along with hadron production systematics.

\section{DIS Model Uncertainty}

DIS interactions contribute $\sim 10 \%$ of the number of interaction candidates to the QE-like sub-samples, and $\sim 25 \%$ to the two track background like sub-sample. Thus the primary way that the DIS model contributes to the measurement of the $M_{A}^{Q E}$ parameter is through the shape of the background correction function. Uncertainties in the DIS model are addressed by considering $\pm 1 \sigma$ changes to the $r_{i j 2}$ and $r_{i j 3}$ parameters of the KNO model of DIS interactions. These parameters increase or decrease the contribution of two pions $\left(r_{i j 2}\right)$, and three pions $\left(r_{i j 3}\right)$ final states to the $Q^{2}$ distributions. The quadratic sum of the DIS model uncertainty contribution to the QE axial-vector mass is ${ }_{-0.025}^{+0.014}$. 

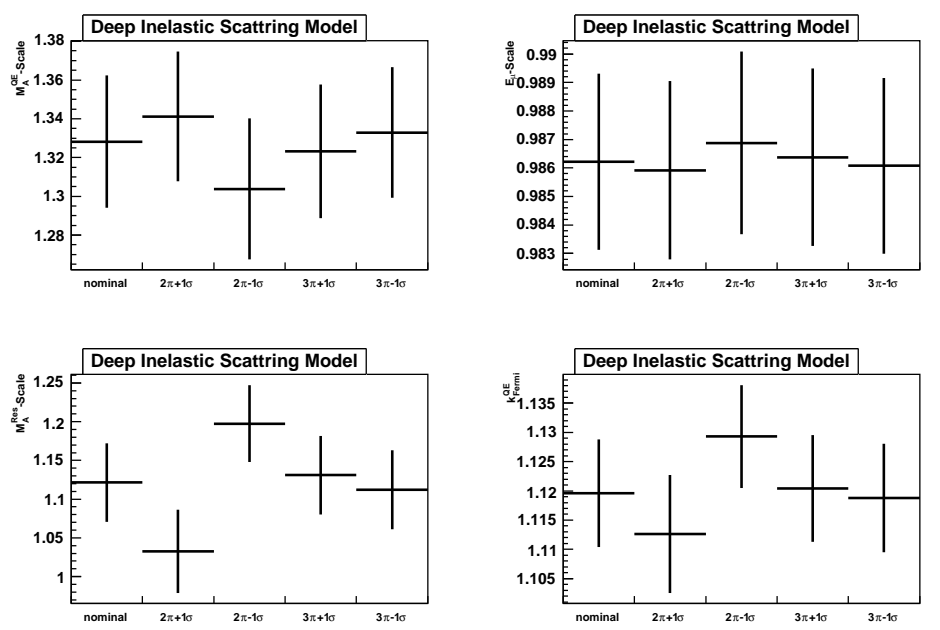

Figure 7.24: Best fit values (along with the HESSE errors) for the DIS model systematic.
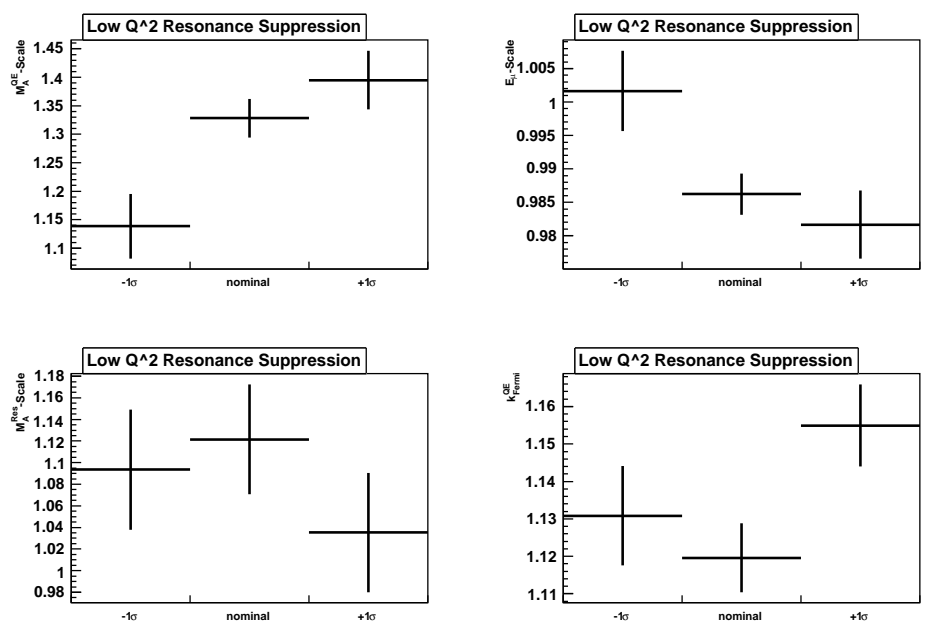

Figure 7.25: Best fit values (along with the HESSE errors) due to the low $Q^{2}$ resonance suppression function. 


\section{Resonance Suppression Function}

The resonance suppression function developed in [88]also addressed a corresponding error band on the low $Q^{2}$ resonances suppression function. This error band includes model uncertainties that include the value of the resonance axial-vector mass $\left(M_{A}^{R E S}\right.$ ), low $Q^{2}$ DIS migration, along with a contribution from alternative models to the suppression function, and other smaller contributions to the error band. The assumed $1 \sigma$ error for this systematic comes from the defined error band. The shift in the best fit values from changing the resonance suppression function are shown in Figure 7.25. The resonance suppression function error band is the largest contributor to the uncertainty in $M_{A}^{Q E}$ (on the minus side of the primary best fit) contributing ${ }_{-0.188}^{+0.066}$ to the $M_{A}^{Q E}$ uncertainty.
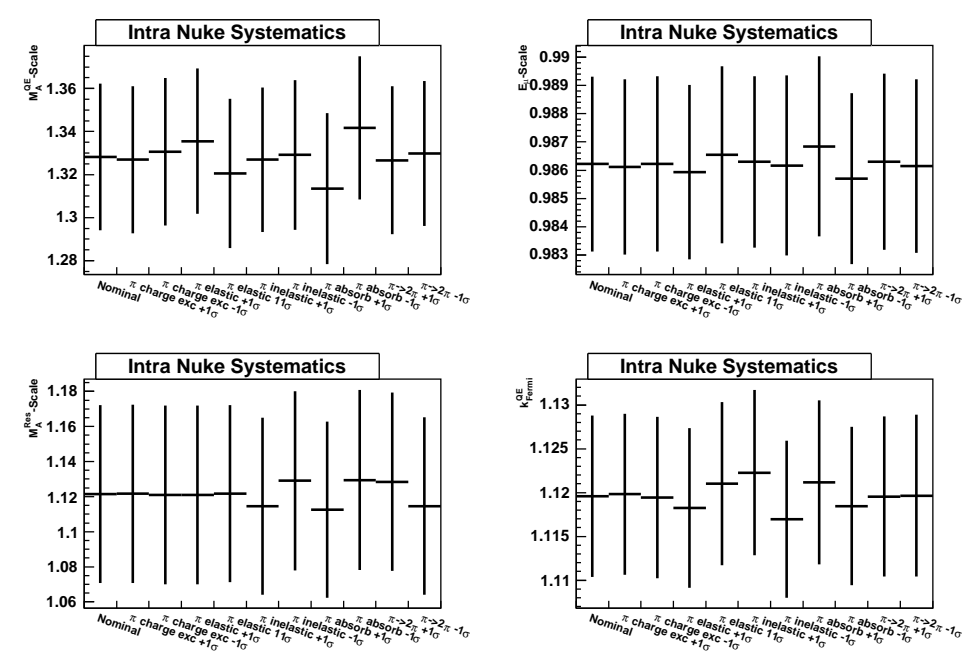

Figure 7.26: Best fit values (along with the HESSE errors) for some of the intranuclear rescattering uncertainties.

\section{Intranuclear Rescattering}

The NEUGEN neutrino event generator, uses the INTRANUKE software package to describe the intranuclear rescattering [80]. Using the INTRANUKE package 
a reweighting procedure was created [81]. This reweighting procedure divides the known possible final states into different branching rations or 'fates.' Uncertainties in physical quantities, such as pion charge exchange, pion absorption, or nucleon knockout, are explored by changing the importance weight of the fate or fates that are effected by the particular physical quantity being examined. Simultaneously all of the other fates are changed in such a manner that the total probability remains unitary. Uncertainties from $\pm 0.25 \sigma$ up to $\pm 2 \sigma$ in the physical quantities can be considered. However only $\pm 1 \sigma$ are considered, unless the $\pm 1 \sigma$ shifts show a large or unusual change in the best fit values. Figures 7.26 and 7.27 show the effect of $\pm 1 \sigma$ shifts on the best fit values. Figure 7.27 shows
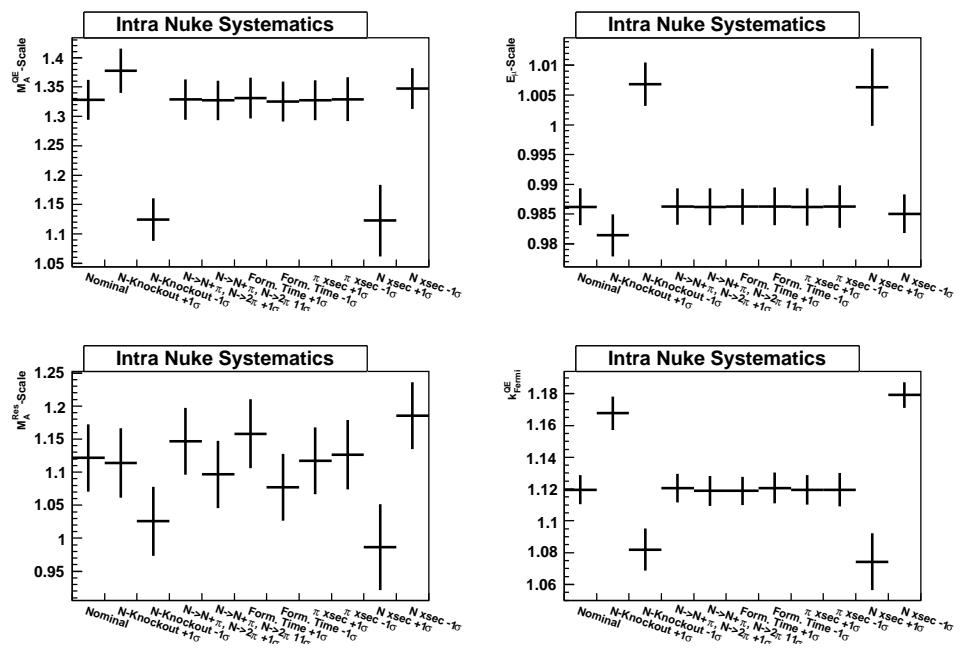

Figure 7.27: Best fit values (along with the HESSE errors) for some of the intranuclear rescattering uncertainties.

that there are two intranuclear rescattering parameters that produce large changes in the best fit values. These parameters control the nucleon knockout and the nucleon cross section. There is a very strong anti-correlation between the nucleon knockout and the nucleon cross section as would be expected from theoretical models. The anti-correlation between nucleon knockout and the nucleon cross section can be understood by considering a single nucleon liberated from the nu- 
cleus as a result of a neutrino scattering interaction. When a nucleon is liberated from the nucleus, the nucleon recoils from the parent collision with significant additional momentum. As the recoil nucleon scatters away from the parent collision the recoil nucleon may re-scatter off of other nucleons within the nucleus. At the lower momentum transfers $(<2 \mathrm{GeV})$ selected in this analysis, every secondary nucleon-nucleon collision divides the originally imparted between both of the nucleons. Increasing the nucleon cross section will increase the number of these nucleon-nucleon interactions. If the original recoil nucleon losses enough momentum due to these nucleon-nucleon interactions, the original recoil nucleon, and likely all of the subsequent nucleons will not leave the nucleus. Thus increasing the nucleon cross section will reduce the number of nucleons in the final state. Figure 7.28 demonstrates this correlation between nucleon knockout and the nucleon cross section.

Further examination of Figure 7.28 also reveals that the low hadronic shower energy sub-sample is more sensitive to the effect of these intranuclear rescattering systematics, than the two track QE-like sub-sample. The low hadronic shower energy sub-sample shows $\sim 5 \%$ change in the $Q_{Q E}^{2}$ distribution while the two track QE-like sample shows an $\sim 2 \%$ change in the $Q_{Q E}^{2}$ distribution (except for the lowest $Q_{Q E}^{2}$ bin). Because the low hadronic shower energy sub-sample is sensitive to these parameters, it is possible to further constrain these parameters, this is done by including these intranuclear rescattering parameters directly in the fit. However this is impossible due to limitations in the INTRANUKE reweighting system. INTRANUKE's reweigting system is configured to conserve probability between the final state branching ratios, if two INTRANUKE reweight parameters are applied simultaneously probability would not be conserved between the final state branching ratios. The alternative to including the parameters directly in the fit is to scan along $\chi^{2}$ for different values of these INTRANUKE systematics. From this scan of the $Q^{2}$ distribution the defined ' $\pm 1 \sigma$ ' uncertainty due to the nucleon knockout and nucleon cross section will be the fractional $1 \sigma$ change that results in a $\Delta \chi^{2}$ of 1 from the minimum $\chi^{2}$. These $\chi^{2}$ scans are shown in 

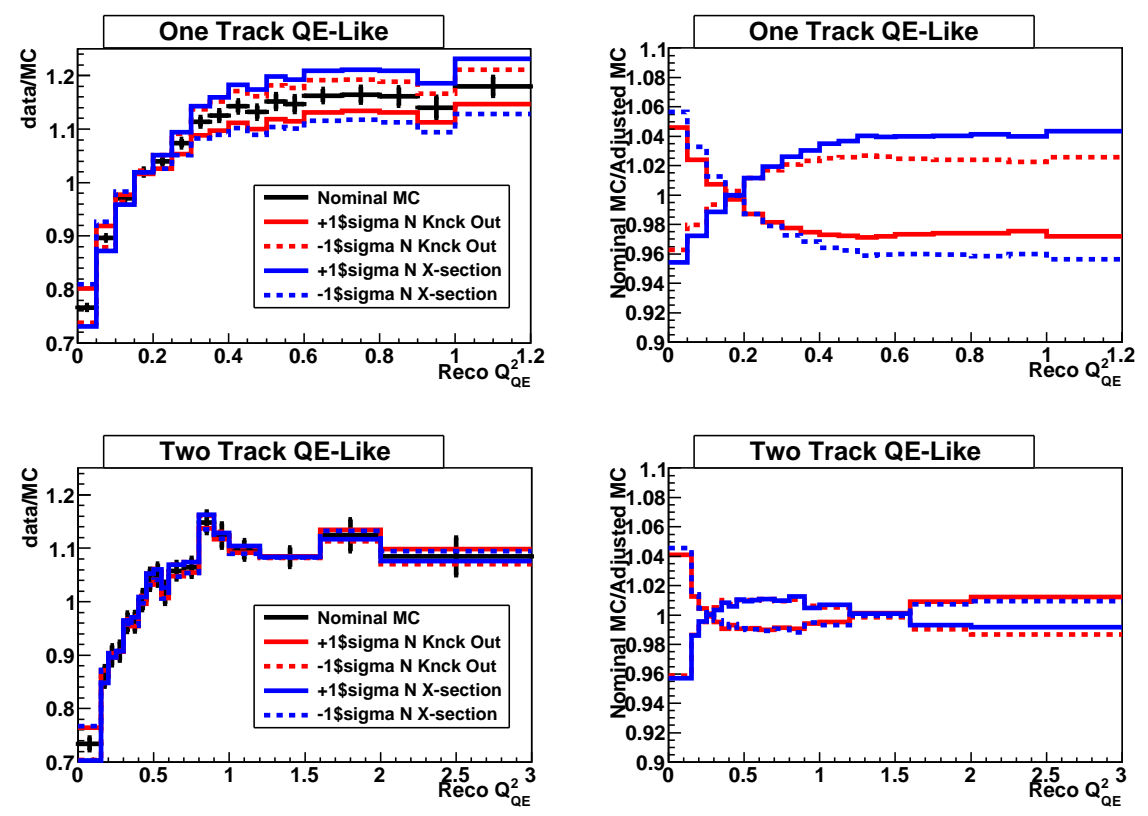

Figure 7.28: Left: the ratio of data to $\mathrm{MC}$ for the nominal $\mathrm{MC}$ along with the $\pm 1 \sigma$ shifts to the nucleon knockout and nucleon cross section intranuclear rescattering systematics applied to the nominal MC. Right: the ratio of the nominal MC to the $\pm 1 \sigma$ nucleon knockout and nucleon cross section intranuclear rescattering systematics applied to the nominal MC. Above: the low hadronic energy QE-like sub-sample. Below: the two track QE-like sub-sample.

Figures 7.29 and 7.30. The uncertainty due to the model of intranuclear rescattering contributes ${ }_{-0.023}^{+0.016}$ to the $M_{A}^{Q E}$ uncertainty. Figures 7.29 and 7.30 both show that the data prefers the nominal MC over any change in the nucleon knockout or nucleon cross section parameters, these Figures also show that a change in these parameters of $\pm 0.25 \sigma$ produce a $\Delta \chi^{2}$ of $\sim 2$. Thus $\pm 0.25 \sigma$ is used as a conservative estimate of the uncertainty in the neutrino cross section fit parameters due to the uncertainty in the nucleon knockout and nucleon cross section INTRANUKE parameters. Figure 7.31 reproduces Figure 7.27 with the new definition of $1 \sigma$ INTRANUKE changes. 


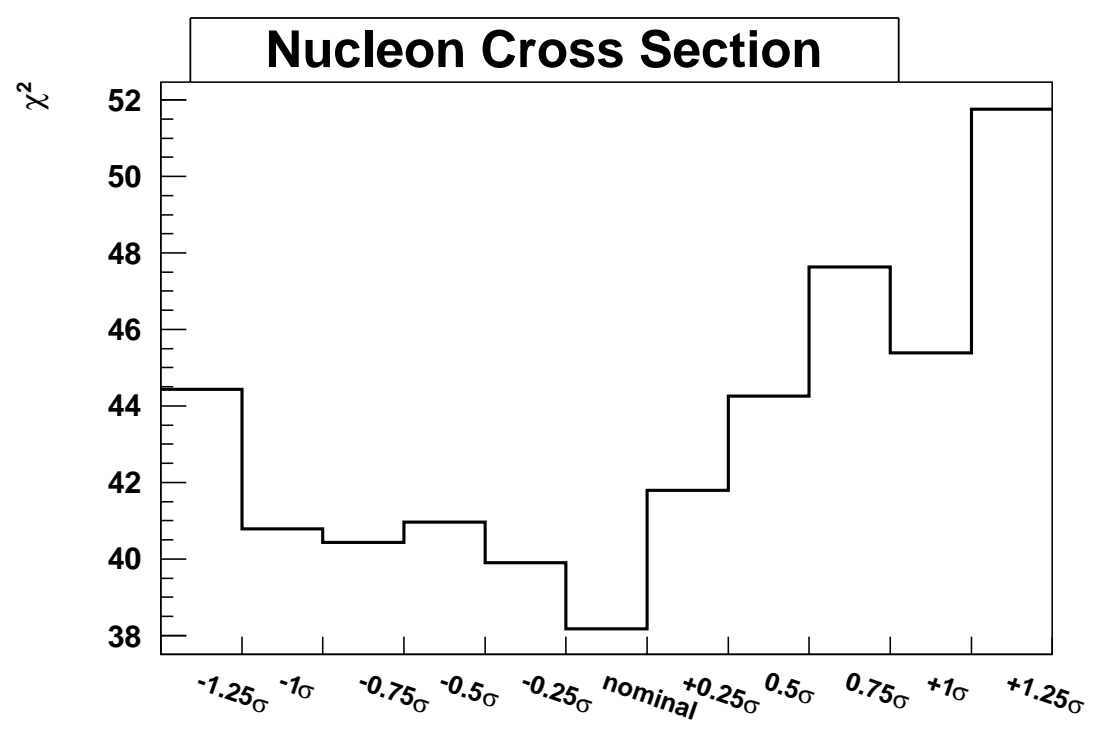

Figure 7.29: $\chi^{2}$ scan of the nucleon cross section INTRANUKE systematic parameter. The $\pm 1 \sigma$ uncertainty due to the nucleon cross section parameter is defined as the fit parameter shift due to a $\Delta \chi^{2}$ from the minimum of 1 .

\begin{tabular}{|l|c|c|}
\hline Systematic & $+M_{A}^{Q E}$ Change & $-M_{A}^{Q E}$ Change \\
\hline \hline Sub-sample Selection Criteria & 0.100 & 0.183 \\
Beam Flux Uncertainty & 0.020 & 0.019 \\
DIS Model Uncertainty & 0.014 & 0.025 \\
Resonance Suppression Function & 0.066 & 0.188 \\
Intranuclear Rescattering & 0.016 & 0.023 \\
\hline Total & 0.123 & 0.265 \\
\hline
\end{tabular}

Table 7.5: Contribution to the systematic error due to the different categories defined in this Section on the $M_{A}^{Q E}$ fit parameter.

\subsubsection{Best Fit Confidence Regions}

The contribution to the confidence interval due to the fit on the best fit values of the fit parameters is calculated by navigating the multivariate $\chi^{2}$ distribution away 


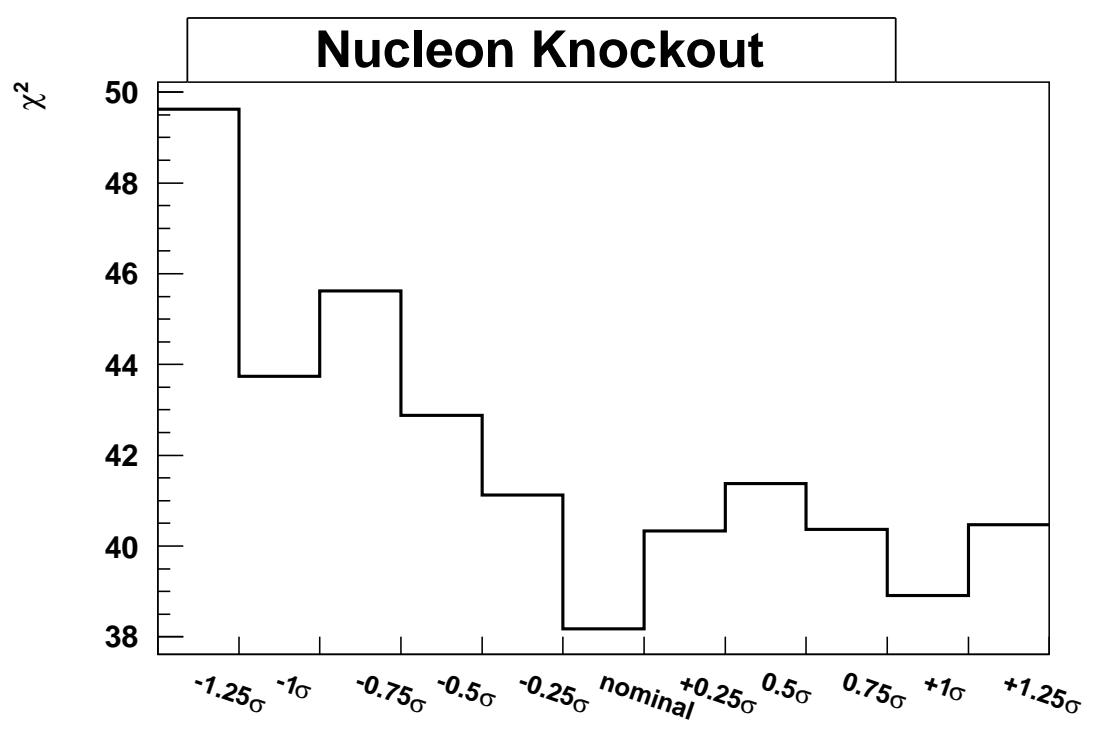

Figure 7.30: $\chi^{2}$ scan of the nucleon knockout INTRANUKE systematic parameter. The $\pm 1 \sigma$ uncertainty due to the nucleon cross section parameter is defined as the fit parameter shift due to a $\Delta \chi^{2}$ from the minimum of 1 .

from the minimum of the $\chi^{2}$ distribution by 1 , this is done with respect to the a single fit parameter while marginalizing with respect to every other fit parameter. A similar procedure is used to define a two parameter confidence region. A contour region is defined inside which the $\chi^{2}$ is 2.3 greater than the minimum $\chi^{2}$. These two dimensional contours will show any correlations between the displayed parameters. Table 7.6 shows the best fit values with along with the contribution to the confidence interval due to the fit and due to the systematics discussed in Section 7.5.4. 

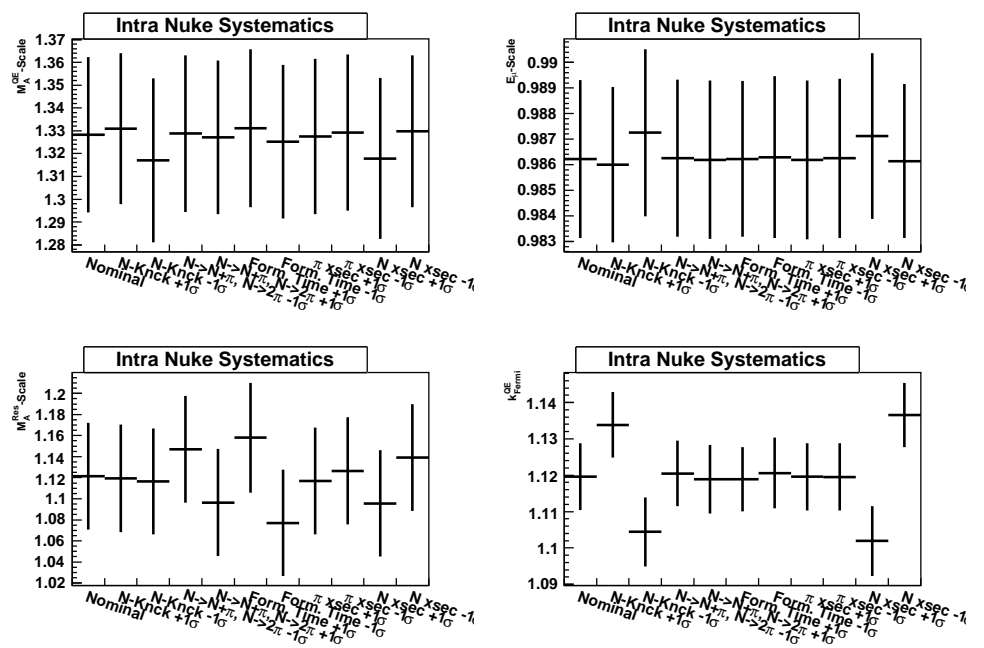

Figure 7.31: Best fit values (along with the HESSE errors) for some of the intranuclear rescattering uncertainties.

\begin{tabular}{|l|c|c|c|}
\hline Parameter & Best Fit Value & Fit Error & Systematic Error \\
\hline \hline$M_{A}^{Q E}(\mathrm{GeV})$ & 1.315 & ${ }_{-0.038}^{+0.037}$ & ${ }_{-0.265}^{+0.123}$ \\
\hline$E_{\mu}$-Scale & 0.986 & ${ }_{-0.004}^{+0.004}$ & ${ }_{-0.008}^{+0.022}$ \\
\hline$M_{A}^{R E S}(\mathrm{GeV})$ & 1.256 & ${ }_{-0.057}^{+0.057}$ & ${ }_{-0.221}^{+0.122}$ \\
\hline$k_{\text {Fermi }}^{Q E}$-Scale & 1.120 & ${ }_{-0.009}^{+0.009}$ & ${ }_{-0.028}^{+0.054}$ \\
\hline$E_{\text {Had }}$-Offset $(\mathrm{GeV})$ & 0.038 & ${ }_{-0.035}^{+0.0035}$ & ${ }_{-0.004}^{+0.000}$ \\
\hline
\end{tabular}

Table 7.6: Best fit values for the along with contributions to the uncertainty due to the fit and the systematics considered in Section 7.5.4. 


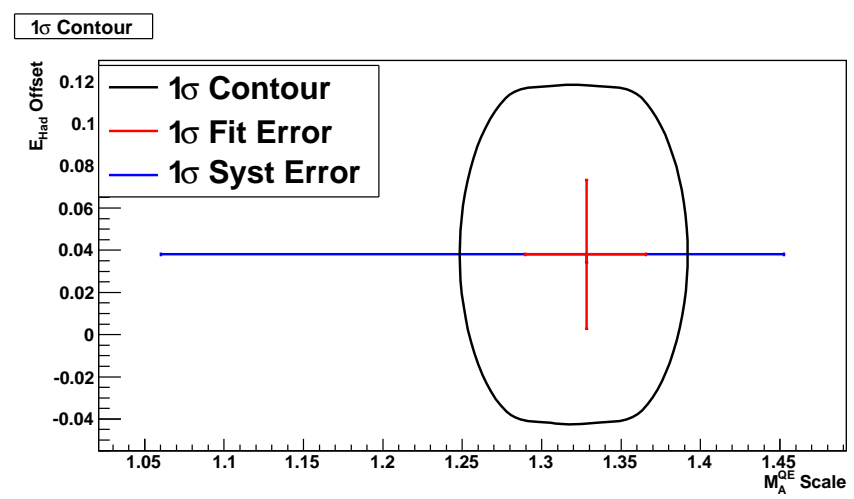

Figure 7.32: One sigma contour of the $M_{A}^{Q E}$ (a scale factor on the nominal value of $M_{A}^{Q E}$ equal to $0.99 \mathrm{GeV}$ ) parameter versus the hadronic energy offset systematic parameter. The hadronic energy offset parameter shows very little correlation with any of the other fit parameters. Thus a plot of any other fit parameter versus the hadronic energy offset will look very similar to this one.

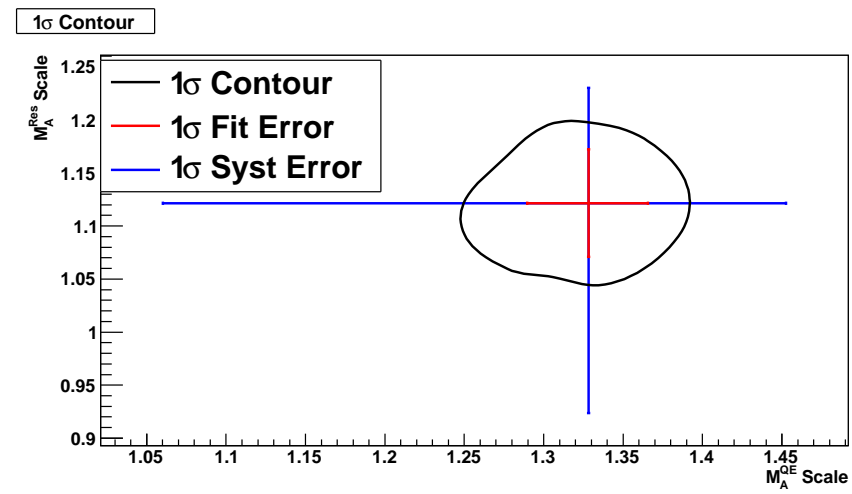

Figure 7.33: One sigma contour of the $M_{A}^{Q E}$ parameter versus the $M_{A}^{R E S}$ parameter. Both of these parameters are scale factors on the nominal values of these parameters. The nominal value for $M_{A}^{Q E}$ is $0.99 \mathrm{GeV}$ while the nominal value for $M_{A}^{R E S}$ is $1.12 \mathrm{GeV}$. The use of the two track QE-like and the two track background like sub-samples breaks the correlations between the both of the axial-vector parameters. 


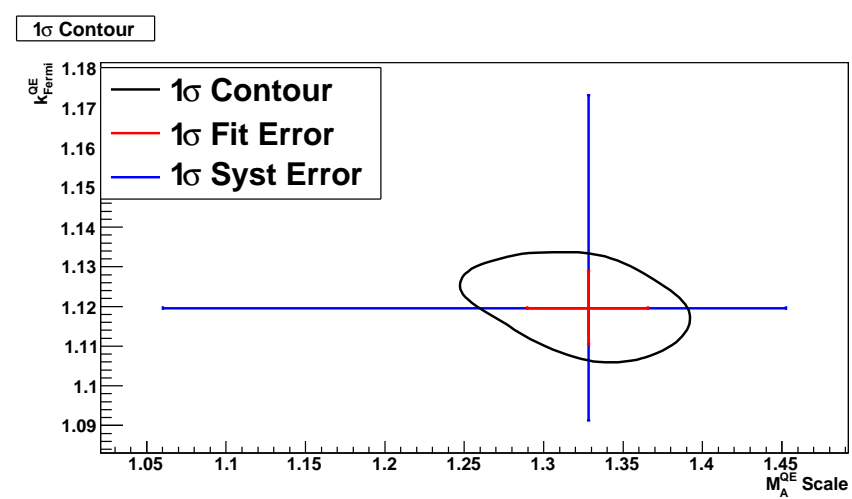

Figure 7.34: One sigma contour of the $M_{A}^{Q E}$ (a scale factor on the nominal value of $M_{A}^{Q E}$ equal to $0.99 \mathrm{GeV}$ ) parameter versus the $k_{F e r m i}^{Q E}$ parameter. The tension between the low hadronic energy offset subsample and the two track QE-like sub-sample largely breaks the correlation between these parameters. There is still some correlation in the lower allowed region of the quasi-elasti axial-vector mass parameter.

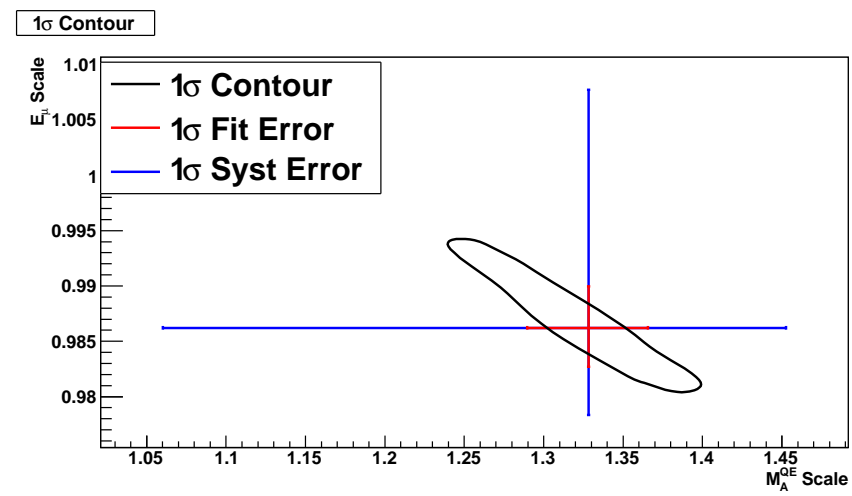

Figure 7.35: One sigma contour of the $M_{A}^{Q E}$ (a scale factor on the nominal value of $M_{A}^{Q E}$ equal to $0.99 \mathrm{GeV}$ ) versus the $E_{\mu}$-scale systematic parameter. There is a strong correlation between these parameters due to the muon energy dependence of $Q_{Q E}^{2}$. 


\subsection{Conclusion}

Using a simultaneous fit of two statistically independent quasi-elastic dominated sub-samples of the MINOS $v_{\mu}$-CC event sample, the quasi-elastic axial-vector mass is measured to be $1.315_{-0.038}^{+0.037}$ (fit) ${ }_{-0.265}^{+0.123}$ (syst.) $(\mathrm{GeV})$. Two sub-samples are used to measure $M_{A}^{Q E}$ and are composed primarily of quasi-elastic interactions, while a third sub-sample which is composed of primarily resonance interactions is used to constrain the behavior of the background. This measurement is dominated by systematic uncertainties of which the largest uncertainties are due to the description of the resonances background and the definition of the low hadronic energy sub-sample. 


\section{Chapter 8}

\section{Interpreting the Results}

The quasi-elastic axial-vector mass $\left(M_{A}^{Q E}\right)$ has been measured to be $1.315_{-0.038}^{+0.037}(\mathrm{fit})_{-0.265}^{+0.123}$ (syst.) $(\mathrm{GeV})$ by using a fit to the shape of the $Q_{Q E}^{2}$ distribution. This fit was performed simultaneously on two statistically independent sub-samples of the $v_{\mu}$-CC sample. A third sub-sample was used to constrain the non-QE background contained within the QE-like sub-samples. This measurement constitutes the first high-statistics measurement of $v_{\mu}$-CC QE scattering off of iron.

This value of $M_{A}^{Q E}$ is $\sim 30 \%$ larger than the global mean (circa 2001) of $1.026 \pm 0.021$. Furthermore this measurement is dominated by systematic uncertainties of which the largest contributions come from the definition of the low hadronic energy sub-sample and the model of the non-QE background.

\subsection{Nuclear Effects}

There are two indications of the presence of correlations between nucleons within the nucleus. These are an additional low momentum nucleon (lower momentum than the recoil nucleon) that recoils in the opposite direction in the zero momentum frame and a transverse enhancement of the neutrino $Q^{2}$ differential cross section. The transverse enhancement of the neutrino $Q^{2}$ differential cross section 
effects the differential cross section in a way that is very similar to an increase in the quasi-elastic axial vector mass. Because MINOS is composed primarily of iron (with many nucleons per nucleus) the effect of these nucleon correlations presumably should be stronger, than for example, on the lower $\mathrm{Z}$ targets of NOMAD and MiniBooNE. However the iron composition of MINOS also negatively impacts the measurement of the additional low momentum nucleon because the dense iron planes cause a high rate of energy loss as a function of distance traveled. Even given the high detection threshold of proton in MINOS, there is still evidence of additional nucleons near the interaction vertex (see Section 6.4.1), however the evidence is limited. It is not really possible to even reconstruct the direction of these nucleon candidates.

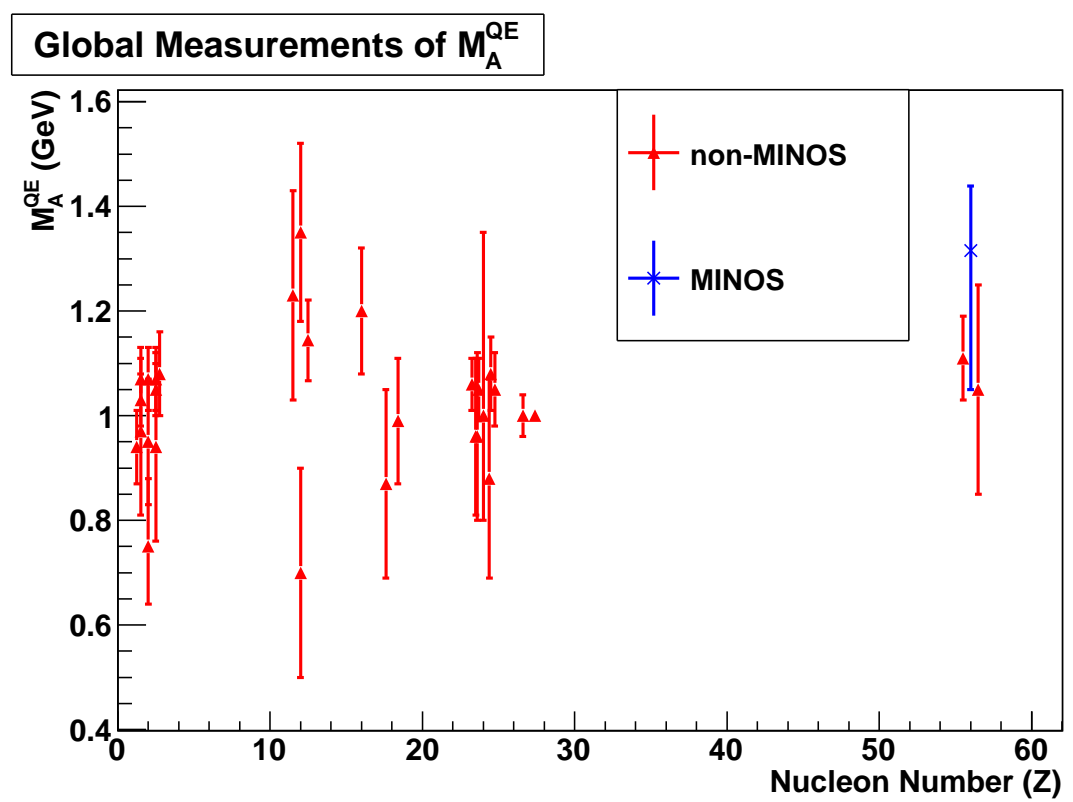

Figure 8.1: Measured $M_{A}^{Q E}$ values as a function of the neutrino target nucleon number. Previous measurements are red, while the measurement presented in here is blue.

MINOS also sees a larger value of the quasi-elastic axial-vector mass parameter. However this measurement is just one of many high statistics measurements 
(low neutrino energy) measurements of $M_{A}^{Q E}$. What makes MINOS unique is the iron target. If there is a nucleon number $(\mathrm{Z})$ dependence to the $M_{A}^{Q E}$ values, high $\mathrm{Z}$ targets are crucial to discovering it. Figure 8.1 shows the global measurements, from Table 3.1 of $M_{A}^{Q E}$ as a function of the neutrino target $Z^{1}$ compared to the measurement of $M_{A}^{Q E}$ measured here. Figure 8.1 shows that there may be a slight upward trend at higher $Z$, though this trend is far from conclusive. More measurements with greater precision are needed to identify this potential trend.

\subsection{Analysis Improvements}

This analysis is systematics limited. Improving this measurement requires improving on the systematic uncertainties. The two systematics that contribute the largest uncertainties to the $M_{A}^{Q E}$ measurement are the definition of the low hadronic energy sub-sample and the correction to the low $Q^{2}$ resonance interactions. Reducing the low hadronic energy systematic requires re-examining the selection criteria used, while reducing the low $Q^{2}$ resonance correction systematic requires a better understanding of the non-QE background.

\subsubsection{Selection Criteria Systematic}

At the generator level the distinction between quasi-elastic, resonance, and DIS scattering can be considered to be a division based on the energy of the hadronic final state. Quasi-elastic interactions have the lowest final state hadron energy of these three types of interactions. Originally the low hadronic energy sub-sample was conceived as an inclusive quasi-elastic sub-sample. Further examination of the unselected interaction candidates revealed that it was possible to select additional quasi-elastics by looking for proton 'tracks' giving the two track QE-like

\footnotetext{
${ }^{1}$ Some of the measurements of $M_{A}^{Q E}$ used a composite of different materials, for these measurements an average $\mathrm{Z}$ was calculated. Several of the measurements list Freon as the target material. Freon is a Dupont brand name for many similar refrigerants, when Freon is listed, $\mathrm{CCl}_{2} \mathrm{~F}_{2}$ is assumed.
} 
sub-sample. This two track QE-like sample was explicitly defined as not low hadronic shower energy $\left(E_{\text {Had }}>250 \mathrm{MeV}\right)$. There remains a 'third' QE-like sample with moderate $Q^{2}$ values between $0.5 \mathrm{GeV}^{2}$ and $1.5 \mathrm{GeV}^{2}$ that is not used in the analysis presented here. These $\mathrm{QE}$ interactions have hadronic energy greater thatn $250 \mathrm{MeV}$, but with a configuration of shower scintillator hits that couldn't be reconstructed into a proton track.
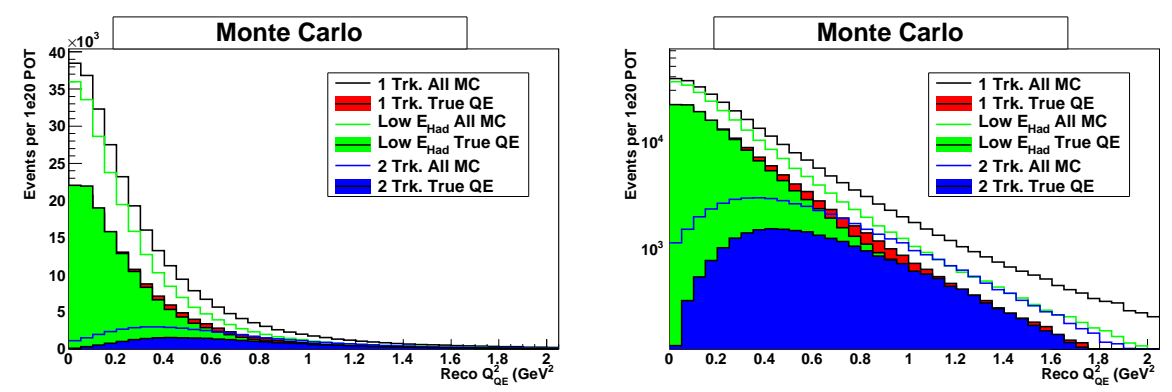

Figure 8.2: Left: $Q^{2}$ distributions used in this analysis along with an alternative to the low hadronic energy sub-sample called the 'one track' sub-sample. Right: the same distributions as the left but on a semi-log scale.

The large region over which the low hadronic energy selection criteria is examined is due to the large uncertainty in the hadronic energy variable (particularly at low hadronic energy). It seems desirable to move away from a sub-sample definition that doesn't use the hadronic energy variable at all. The first selection criteria for the two track sub-samples is the presence of a proton-like track in the hadron shower. The criteria for a proton-like track is deliberately set very low. It is relatively easy to reject non- $\mathrm{QE}$ interactions once a candidate proton track has been found.

An alternative selection scheme is to select based on a one/two track duality. Instead of examining the hadronic shower energy first and using everything with less than $250 \mathrm{MeV}$. Then examining the interaction candidates with hadronic shower energy greater than $250 \mathrm{MeV}$ for a proton track. Why not use the presence 
of the proton track as the first criteria? Figure 8.2 shows the $Q_{Q E}^{2}$ distribution for an alternative selection to the low hadronic energy sub-sample along with the two QE-like sub-samples used in this analysis: the low hadronic energy sub-sample, and the two track QE-like sub-sample. This alternative to the low hadronic energy sub-sample is a 'true' one track sub-sample. The alternative selection loses a little bit in purity at higher $Q^{2}$ but is more easily defined, and is not dependent on hadronic energy variable.

\subsubsection{Background Modeling}

The low $Q^{2}$ correction function from [88] is defined using an ad hoc fit procedure to develop a smooth suppression function that suppress the resonances only. The resonances are further constrained by the two track background-like sub-sample. Both of these suppression techniques are data driven and ultimately improve the quality of the fit. The low $Q^{2}$ correction function has a very conservatively defined error band in part drive by inherent uncertainty in the 'by-hand' fitting procedure that was used. Furthermore the interaction candidate sub-samples that were used to generate the low $Q^{2}$ correction function had a large amount of contamination from both QE and DIS interactions.

There is ample evidence that the neutrino interaction simulators do not correctly simulate single pion production (in NEUGEN this is the resonance interaction). This evidence includes [88] but also [34] and [92] and, of course, this analysis. In [92], the author shows that modifications to the single $\pi$ production form factors of Rein-Sehgal which incorporate electron scattering data are better fit to MiniBooNE CC1 $\pi$ sub-sample. Such a procedure has not been attempted on the MINOS MC. Applying a similar procedure to the MINOS MC would provide an additional constraint to the resonance dominated sub-sample and allow for a reduction in the error band associated with the low $Q^{2}$ resonances suppression function. 


\section{Bibliography}

[1] W. Pauli. Dear radioactive ladies and gentlemen. Phys. Today, 31N9:27, 1978.

[2] F. Reines and C.L. Cowan. Detection of the free neutrino. Phys. Rev., 92:830-831, 1953.

[3] R. Davis. A review of the Homestake solar neutrino experiment. Prog. Part. Nucl. Phys., 32:13-32, 1994.

[4] Jr. Davis, Raymond, Don S. Harmer, and Kenneth C. Hoffman. Search for neutrinos from the sun. Phys. Rev. Lett., 20:1205-1209, 1968.

[5] R. Davis. Solar neutrinos. II: Experimental. Phys. Rev. Lett., 12:303-305, 1964.

[6] R. Becker-Szendy, C.B. Bratton, D. Casper, S.T. Dye, W. Gajewski, et al. The Electron-neutrino and muon-neutrino content of the atmospheric flux. Phys. Rev., D46:3720-3724, 1992.

[7] K.S. Hirata et al. Observation of a small atmospheric muon-neutrino electron-neutrino ratio in Kamiokande. Phys. Lett., B280:146-152, 1992.

[8] W.W.M. Allison et al. The Atmospheric neutrino flavor ratio from a 3.9 fiducial kiloton year exposure of Soudan-2. Phys. Lett., B449:137-144, 1999.

[9] B. Pontecorvo. Neutrino Experiments and the Problem of Conservation of Leptonic Charge. Sov. Phys. JETP, 26:984-988, 1968. 
[10] P. Adamson et al. Improved search for muon-neutrino to electron-neutrino oscillations in MINOS. Phys. Rev. Lett., 2011. * Temporary entry *.

[11] Vernon D. Barger, J.G. Learned, P. Lipari, Maurizio Lusignoli, S. Pakvasa, et al. Neutrino decay and atmospheric neutrinos. Phys. Lett., B462:109-114, 1999.

[12] E. Lisi, A. Marrone, and D. Montanino. Probing possible decoherence effects in atmospheric neutrino oscillations. Phys. Rev. Lett., 85:1166-1169, 2000 .

[13] Y. Ashie et al. Evidence for an oscillatory signature in atmospheric neutrino oscillation. Phys. Rev. Lett., 93:101801, 2004.

[14] P. Adamson et al. Measurement of Neutrino Oscillations with the MINOS Detectors in the NuMI Beam. Phys. Rev. Lett., 101:131802, 2008.

[15] T.D. Lee and Chen-Ning Yang. Question of Parity Conservation in Weak Interactions. Phys. Rev., 104:254-258, 1956.

[16] C.S. Wu, E. Ambler, R.W. Hayward, D.D. Hoppes, and R.P. Hudson. EXPERIMENTAL TEST OF PARITY CONSERVATION IN BETA DECAY. Phys. Rev., 105:1413-1414, 1957.

[17] M. Goldhaber, L. Grodzins, and A.W. Sunyar. HELICITY OF NEUTRINOS. Phys. Rev., 109:1015-1017, 1958.

[18] S.L. Glashow. Partial Symmetries of Weak Interactions. Nucl.Phys., 22:579_ $588,1961$.

[19] Steven Weinberg. A Model of Leptons. Phys. Rev. Lett., 19:1264-1266, 1967.

[20] Abdus Salam. Weak and Electromagnetic Interactions. pages 367-377, 1968. 
[21] F.J. Hasert et al. Observation of Neutrino Like Interactions Without Muon Or Electron in the Gargamelle Neutrino Experiment. Phys. Lett., B46:138-140, 1973.

[22] R.P. Feynman and Murray Gell-Mann. Theory of Fermi interaction. Phys. Rev., 109:193-198, 1958.

[23] Nicola Cabibbo. Unitary Symmetry and Leptonic Decays. Phys. Rev. Lett., 10:531-533, 1963.

[24] Makoto Kobayashi and Toshihide Maskawa. CP Violation in the Renormalizable Theory of Weak Interaction. Prog. Theor. Phys., 49:652-657, 1973.

[25] C.H. Llewellyn Smith. Neutrino Reactions at Accelerator Energies. Phys. Rept., 3:261-379, 1972.

[26] M.N. Rosenbluth. High Energy Elastic Scattering of Electrons on Protons. Phys. Rev., 79:615-619, 1950.

[27] Michael E. Peskin and Daniel V. Schroeder. An Introduction to quantum field theory. 1995 .

[28] R.G. Sachs. High-Energy Behavior of Nucleon Electromagnetic Form Factors. Phys. Rev., 126:2256-2260, 1962.

[29] R.A. Smith and E.J. Moniz. NEUTRINO REACTIONS ON NUCLEAR TARGETS. Nucl. Phys., B43:605, 1972.

[30] M. Dorman. Preliminary results for CCQE scattering with the MINOS near detector. AIP Conf. Proc., 1189:133-138, 2009.

[31] A.A. Aguilar-Arevalo et al. Measurement of muon neutrino quasi-elastic scattering on carbon. Phys. Rev. Lett., 100:032301, 2008. 
[32] Omar Benhar, Nicola Farina, Hiroki Nakamura, Makoto Sakuda, and Ryoichi Seki. Electron- and neutrino-nucleus scattering in the impulse approximation regime. Phys. Rev., D72:053005, 2005.

[33] H. Gallagher. The NEUGEN neutrino event generator. Nucl. Phys. Proc. Suppl., 112:188-194, 2002.

[34] A.A. Aguilar-Arevalo et al. First Measurement of the Muon Neutrino Charged Current Quasielastic Double Differential Cross Section. Phys. Rev., D81:092005, 2010.

[35] M. Martini, M. Ericson, G. Chanfray, and J. Marteau. A unified approach for nucleon knock-out, coherent and incoherent pion production in neutrino interactions with nuclei. Phys. Rev., C80:065501, 2009.

[36] Howard Scott Budd, A. Bodek, and J. Arrington. Modeling quasielastic form-factors for electron and neutrino scattering. 2003.

[37] R. Bradford, A. Bodek, Howard Scott Budd, and J. Arrington. A New parameterization of the nucleon elastic form-factors. Nucl. Phys. Proc. Suppl., 159:127-132, 2006.

[38] C. Andreopoulos, A. Bell, D. Bhattacharya, F. Cavanna, J. Dobson, et al. The GENIE Neutrino Monte Carlo Generator. Nucl. Instru. Meth., A614:87-104, 2010 .

[39] R.L. Kustom, D.E. Lundquist, T.B. Novey, A. Yokosawa, and F. Chilton. Quasielastic neutrino scattering. Phys. Rev. Lett., 22:1014-1017, 1969.

[40] W.A. Mann, U. Mehtani, B. Musgrave, Y. Oren, P.A. Schreiner, et al. Study of the reaction $v n \rightarrow \mu^{-}$p. Phys. Rev. Lett., 31:844-847, 1973.

[41] S.J. Barish, J. Campbell, G. Charlton, Y. Cho, M. Derrick, et al. Study of Neutrino Interactions in Hydrogen and Deuterium. 1. Description of the 
Experiment and Study of the Reaction $v d \rightarrow \mu^{-} p p_{s}$. Phys. Rev., D16:3103, 1977.

[42] K.L. Miller, S.J. Barish, A. Engler, R.W. Kraemer, B.J. Stacey, et al. STUDY OF THE REACTION: $v_{\mu} d \rightarrow \mu p p_{S}$. Phys. Rev., D26:537-542, 1982.

[43] N.J. Baker, A.M. Cnops, P.L. Connolly, S.A. Kahn, H.G. Kirk, et al. Quasielastic Neutrino Scattering: A Measurement of the Weak Nucleon Axial Vector Form-Factor. Phys. Rev., D23:2499-2505, 1981.

[44] T. Kitagaki, S. Tanaka, H. Yuta, K. Abe, K. Hasegawa, et al. High-Energy Quasielastic $v_{\mu} n \rightarrow m u^{-} p$ Scattering in Deuterium. Phys. Rev., D28:436442, 1983.

[45] T. Kitagaki, H. Yuta, S. Tanaka, A. Yamaguchi, K. Abe, et al. Study of $v d \rightarrow \mu^{-} p p(s)$ and $v d \rightarrow \mu^{-} \Delta^{++}(1232) n_{s}$ using the BNL 7-foot deuterium filled bubble chamber. Phys. Rev., D42:1331-1338, 1990.

[46] Narumon Suwonjandee. The measurement of the quasi-elastic neutrinonucleon scattering cross section at the Tevatron. 2004. Ph.D. Thesis (Advisor: Randy Johnson).

[47] M.M. Block, H. Burmeister, D.C. Cundy, B. Eiben, C. Franzinetti, et al. Neutrino interactions in the CERN heavy liquid bubble chamber. Phys. Lett., 12:281-285, 1964.

[48] I. Budagov, D.C. Cundy, C. Franzinetti, W.B. Fretter, H.W.K. Hopkins, et al. A study of the elastic neutrino process $v+n \rightarrow \mu^{-}+p$. Lett. Nuovo Cim., 2:689-695, 1969.

[49] S. Bonetti, G. Carnesecchi, D. Cavalli, P. Negri, A. Pullia, et al. Study of Quasielastic Reactions of Neutrino and anti-neutrino in Gargamelle. Nuovo Cim., A38:260-270, 1977. 
[50] M. Pohl et al. EXPERIMENTAL STUDY OF THE REACTION $v n \rightarrow \mu^{-} p$. Lett. Nuovo Cim., 26:332-336, 1979.

[51] D. Allasia, C. Angelini, G.W. van Apeldoorn, A. Baldini, S.M. Barlag, et al. Investigation of exclusive channels in neutrino / anti-neutrino deuteron charged current interactions. Nucl. Phys., B343:285-309, 1990.

[52] V Lyubushkin et al. A Study of quasi-elastic muon neutrino and antineutrino scattering in the NOMAD experiment. Eur. Phys. J., C63:355-381, 2009.

[53] S.V. Belikov, A.P. Bugorsky, V.Sh. Epshtein, V.S. Kaftanov, N.V. Kalganov, et al. QUASIELASTIC muon-neutrino n SCATTERING AT 3-GeV TO 30GeV ENERGY. Yad. Fiz., 35:59, 1982.

[54] S.V. Belikov, A.P. Bugorsky, V.Sh. Epshtein, N.V. Kalganov, L.A. Klimenko, et al. QUASIELASTIC NEUTRINO AND ANTI-NEUTRINOS SCATTERING: TOTAL CROSS-SECTIONS, AXIAL VECTOR FORM-FACTOR. Z. Phys., A320:625, 1985.

[55] H.J. Grabosch, K.K. Kaufmann, U. Krecker, R. Nahnhauer, Z. Nowak, et al. STUDY OF QUASIELASTIC REACTIONS $v n \rightarrow \mu^{-} p$ AND $\bar{v} p \rightarrow \mu^{+} n$ IN BUBBLE CHAMBER SKAT AT 3-GeV TO 20-GeV. Sov. J. Nucl. Phys., 47:1032-1034, 1988.

[56] J. Brunner et al. QUASIELASTIC NUCLEON AND HYPERON PRODUCTION BY NEUTRINOS AND ANTI-NEUTRINOS WITH ENERGIES BELOW 30-GeV. Z. Phys., C45:551, 1990.

[57] R. Gran et al. Measurement of the quasi-elastic axial vector mass in neutrinooxygen interactions. Phys. Rev., D74:052002, 2006.

[58] C. Mariani. K2K recent results. AIP Conf. Proc., 981:247-249, 2008. 
[59] Q. Wu et al. A Precise measurement of the muon neutrino-nucleon inclusive charged current cross-section off an isoscalar target in the energy range $2.5<$ $E_{\mathrm{v}}<40 \mathrm{GeV}$ by NOMAD. Phys. Lett., B660:19-25, 2008.

[60] A.A. Aguilar-Arevalo et al. Unexplained Excess of Electron-Like Events From a 1-GeV Neutrino Beam. Phys. Rev. Lett., 102:101802, 2009.

[61] L.B. Auerbach et al. Measurements of charged current reactions of muon neutrinos on C-12. Phys. Rev., C66:015501, 2002.

[62] Arie Bodek and Howard Budd. Neutrino Quasielastic Scattering on Nuclear Targets: Parametrizing Transverse Enhancement (Meson Exchange Currents). Eur. Phys. J., C71:1726, 2011. * Temporary entry *.

[63] The MINOS Collaboration. The MINOS Technical Design Report. Technical Design Report, 1999.

[64] The MINOS Collaboration. The NuMI Beam Technical Design Report. Technical Design Report, 1999.

[65] D.G. Michael et al. The Magnetized steel and scintillator calorimeters of the MINOS experiment. Nucl. Instrum. Meth., A596:190-228, 2008.

[66] Fermilab Visual Media Services. http://www-visualmedia.fnal.gov.

[67] Donald E. Groom, Nikolai V. Mokhov, and Sergei I. Striganov. Muon stopping power and range tables $10-\mathrm{MeV}$ to $100-\mathrm{TeV}$. Atom. Data Nucl. Data Tabl., 78:183-356, 2001.

[68] K. Nakamura et al. Review of particle physics. J. Phys. G, G37:075021, 2010.

[69] John Stuart Marshall. A study of muon neutrino disappearance with the MINOS detectors and the NuMI neutrino beam. 2008. Ph.D. Thesis (advisor: Mark A. Thomson). 
[70] R. Fruhwirth. Application of Kalman filtering to track and vertex fitting. Nucl. Instrum. Meth., A262:444-450, 1987.

[71] P. Adamson et al. A Study of Muon Neutrino Disappearance Using the Fermilab Main Injector Neutrino Beam. Phys. Rev., D77:072002, 2008.

[72] Jeffrey J. Hartnell. Measurement of the calorimetric energy scale in MINOS. 2005. Ph.D. Thesis (Advisors: Alfons Weber and Geoff Pearce).

[73] C. Zeitnitz and T.A. Gabriel. The GEANT - CALOR interface and benchmark calculations of ZEUS test calorimeters. Nucl. Instrum. Meth., A349:106-111, 1994.

[74] Rene Brun, Federico Carminati, and Simone Giani. GEANT Detector Description and Simulation Tool. 1994. CERN Program Library Long Writeup.

[75] Dieter Rein and Lalit M. Sehgal. Neutrino Excitation of Baryon Resonances and Single Pion Production. Annals Phys., 133:79-153, 1981.

[76] A. Bodek and U.K. Yang. Unified approach for modelling neutrino and electron nucleon scattering cross sections from very high $\mathrm{Q}^{* * 2}$ to $\mathrm{Q}^{* * 2} 2=0$. AIP Conf. Proc., 721:358-362, 2004.

[77] T. Yang, C. Andreopoulos, H. Gallagher, and P. Kehayias. A hadronization model for the MINOS experiment. AIP Conf. Proc., 967:269-275, 2007.

[78] Z. Koba, Holger Bech Nielsen, and P. Olesen. Scaling of multiplicity distributions in high-energy hadron collisions. Nucl. Phys., B40:317-334, 1972.

[79] Torbjorn Sjostrand, Stephen Mrenna, and Peter Z. Skands. PYTHIA 6.4 Physics and Manual. JHEP, 0605:026, 2006.

[80] Steven Dytman. Final state interactions in neutrino-nucleus experiments. Acta Phys. Polon., B40:2445-2460, 2009. 
[81] Mike Kordosky. A procedure to re-weight events to account for uncertainties in final state interactions. MINOS Internal Document, DocDB: 3449-v2:126, 2007.

[82] Mark Dorman. Beam Fit Position Paper. MINOS Internal Document, DocDB: 7146-v1:1-44, 2010.

[83] C. Alt et al. Inclusive production of charged pions in $\mathrm{p}+\mathrm{C}$ collisions at 158 GeV/c beam momentum. Eur. Phys. J., C49:897-917, 2007.

[84] M. Bonesini, A. Marchionni, F. Pietropaolo, and T. Tabarelli de Fatis. On Particle production for high-energy neutrino beams. Eur. Phys. J., C20:1327, 2001.

[85] Rustem Ospanov. A measurement of muon neutrino disappearance with the MINOS detectors and NuMI beam. 2008. Ph.D. Thesis (Advisor: Karol Lang).

[86] R. Ospanov and K. Lang. Alignment of the Near Detector Scintillator Modules Using Cosmic Ray Muons. MINOS Internal Document, DocDB: 1101v2:1-27, 2005.

[87] M. Dorman. Preliminary results for CCQE scattering with the MINOS near detector. AIP Conf. Proc., 1189:133-138, 2009.

[88] N. Graf and R. Gran. Position Paper:Tuning the Resonance Q2 spectrum With MINOS Near Detector Data. MINOS Internal Document, DocDB: 7684-v5:1-22, 2011.

[89] H. Gallagher. Collection of Plots. MINOS Internal Document, DocDB: $7668,2010$.

[90] Ch. Berger and L.M. Sehgal. Lepton mass effects in single pion production by neutrinos. Phys. Rev., D76:113004, 2007. 
[91] M Dorman, R. Gran, N. Mayer, and M Kordosky. Quasielastic Analysis Position Paper. MINOS Internal Document, DocDB: 5909-v7:1-51, 2009.

[92] Jaroslaw A. Nowak. Four Momentum Transfer Discrepancy in the Charged Current pi+ Production in the MiniBooNE: Data versus Theory. AIP Conf. Proc., 1189:243-248, 2009. 